\title{
New brown dwarf candidates in the Pleiades *
}

\author{
T. Eisenbeiss ${ }^{1 \star \star}$, M. Moualla ${ }^{1}$, M. Mugrauer ${ }^{1}$, T. O. B. Schmidt ${ }^{1}$, St. Raetz ${ }^{1}$, R. Neuhäuser ${ }^{1}$, Ch. \\ Ginski $^{1}$, M. M. Hohle ${ }^{1,2}$, A. Koeltzsch ${ }^{1}$, C. Marka ${ }^{1}$, W. Rammo ${ }^{1}$, A. Reithe ${ }^{1}$, T. Roell ${ }^{1}$, and M. Vaňko ${ }^{1}$ \\ 1 Astrophysikalisches Institut und Universitäts-Sternwarte Jena, Schillergässchen 2-3, 07745 Jena, Germany \\ 2 Max Planck Institut für extraterrestrische Physik Garching, Giessenbachstrasse, 85738 Garching
}

Received R-date, accepted A-date

Published online P-date

\begin{abstract}
We have performed deep, wide-field imaging on a $\sim 0.4 \mathrm{deg}^{2}$ field in the Pleiades (Melotte 22). The selected field was not yet target of a deep search for low mass stars and brown dwarfs. Our limiting magnitudes are $R \sim 22 \mathrm{mag}$ and $I \sim 20 \mathrm{mag}$, sufficient to detect brown dwarf candidates down to $40 M_{J}$. We found 197 objects, whose location in the $(I, R-I)$ color magnitude diagram is consistent with the age and the distance of the Pleiades. Using CTK $R$ and $I$ as well as JHK photometry from our data and the 2MASS survey we were able to identify 7 new brown dwarf candidates. We present our data reduction technique, which enables us to resample, calibrate, and co-add many images by just two steps. We estimate the interstellar extinction and the spectral type from our optical and the NIR data using a 2-dimensional $\chi^{2}$ fitting.
\end{abstract}

(C) Year of publication WILEY-VCH Verlag GmbH \& Co. KGaA, Weinheim

\section{Introduction}

Imaging surveys for brown dwarfs (BDs) have often targeted young open clusters or star-forming regions because their ages and distances are known and because BDs are brighter and warmer when they are young (e.g. Bouvier et al., 1998; Moraux et al., 2003). The first BD in the Pleiades, PPl 15 was detected by Basri, Marcy, \& Graham (1996) applying the lithium test method. Soon afterward the BDs Teide 1 and Calar 3 (Rebolo et al. 1996) were detected. According to the number of published papers the Pleiades cluster is the preferred ground for hunting brown dwarfs due to its nearly ideal properties. Those properties are: (a) its age of about 119 Myr (Martín \& Dahm, 2001), where objects at the hydrogen burning mass limit (HBML) are still relatively warm; (b) its distance of about $135.5 \mathrm{pc}$ (An et al., 2007) which is near enough that the apparent magnitude of HBML objects is bright enough to be observed even with relatively small telescopes; (c) its richness, with about 2100 known stars and brown dwarfs (Mermilliod, 1998) and (d) its relatively high galactic latitude, which minimizes the number of background objects.

We selected a field of the Pleiades and did deep imaging in two bands to detect faint, red objects which could be brown dwarfs or low mass stars. We describe our observations in section 2. Data reduction is described in section 3. photometric analysis is given in section 4 The $\chi^{2}$ fitting algorithm of the interstellar extinction and resulting candidates are presented in section 5 and discussed in section 6 .

\footnotetext{
* Based on observations obtained with telescopes of the University Observatory Jena, which is operated by the Astrophysical Institute of the Friedrich- Schiller-University.

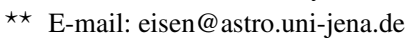

\section{Observations}

Starting in March 2007 we have observed a field at the edge of the Pleiades cluster, which was not a target of extended surveys before (see Tbl. 1 in Schwartz, \& Becklin 2005). The field is located at $\alpha=3^{h} 42^{m} 20.6^{s}, \delta=$ $+25^{\circ} 36^{\prime} 54^{\prime \prime}$. For these observations we have used the Cassegrain-Teleskop-Kamera (CTK) which is installed at the $250 \mathrm{~mm}$ Cassegrain auxiliary telescope of the $900 \mathrm{~mm}$ reflector which is operated at University Observatory Jena. The instrumental details are described in Mugrauer (2009). With its $1024 \times 1024$ Pixel CCD detector and a resolution of about $2.2 \mathrm{arcsec} / \mathrm{pixel}$ the CTK covers a field of view $(\mathrm{FoV})$ of $\sim 38^{\prime} \times 38^{\prime}$. In total we have collected 599 images in $R$ and 781 images in $I$ which yields to a total integration time $36 \mathrm{ks}$ and $47 \mathrm{ks}$ respectively (Fig. 1 and Fig. 2), see Tbl. 1. The majority of the $R$ band images was taken at high airmasses, so the photometric quality of these data is lower than that of the $I$ band data. Nevertheless, the quantum efficiency of the detector is better in $R$ than in $I$. Considering this, the different observing conditions in both observation campaigns ( $R$ and $I$ ), and regarding our special interest in red objects the total integration time gives a comparable sensitivity in the co-added images.

\section{Data reduction}

The individual integration time per image was $60 \mathrm{~s}$ in $R$ and $I$, because we want to study both, faint objects as well as their photometric variability. The latter is still under investigation and will be presented later elsewhere (Moualla et al. in prep). 


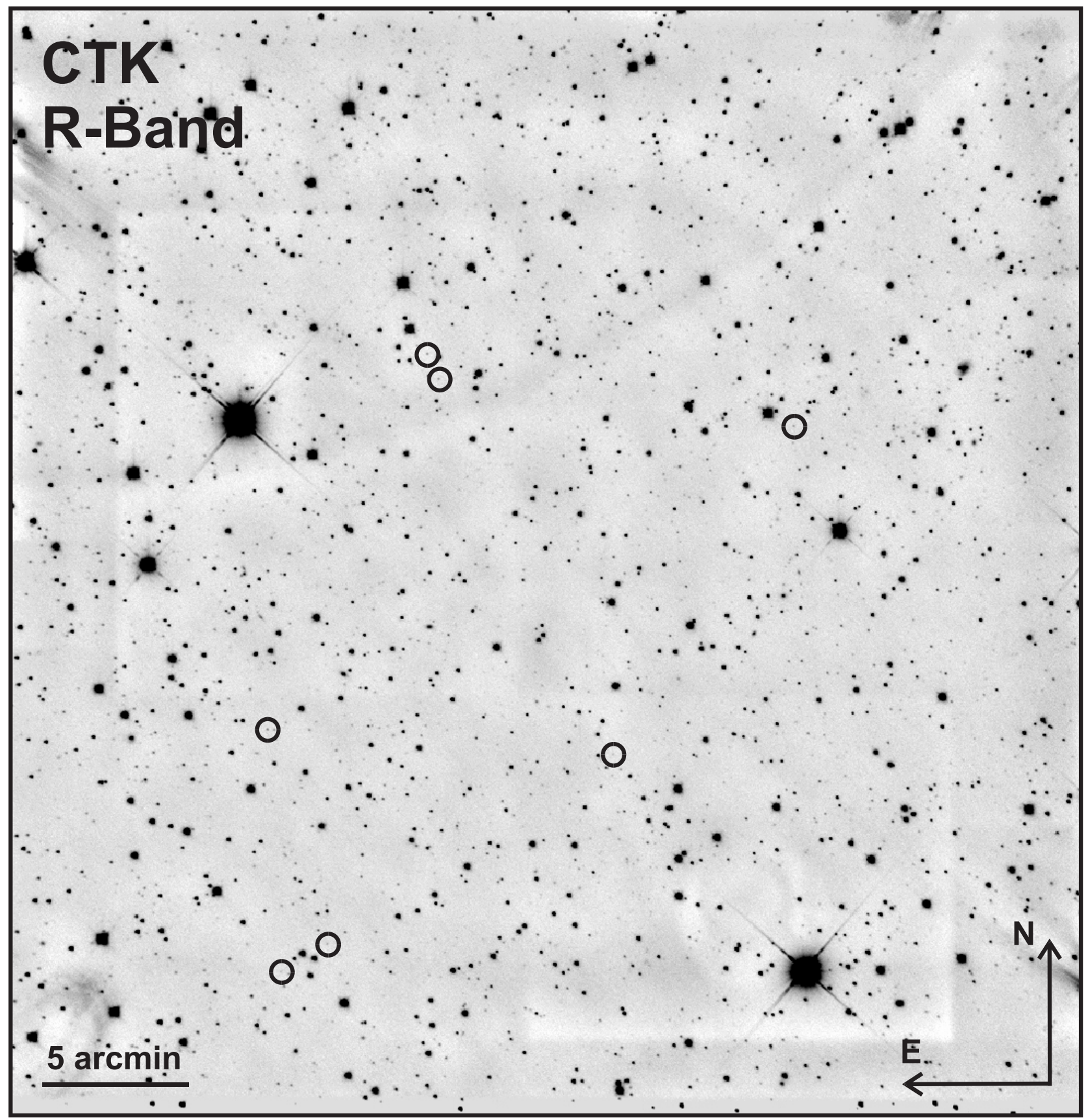

Abbildung 1 Our observed field. The summed up $R$ Filter image with a total exposure time of $36 \mathrm{ks}$ is shown. The center of the field is $\alpha=3^{h} 42^{m} 20.6^{s}, \delta=+25^{\circ} 36^{\prime} 54^{\prime \prime}$. North is up east is left. The brown dwarf candidates we have detected are marked by circles, see also Fig. 10 to Fig. 14. The illumination effects at the edges of the image are reflections of bright stars. The total covered field in this image is about $36.5 \times 37.7 \mathrm{arcmin}^{2}$. The two bright and saturated stars are the F5 star HD 23075 (NE) and the K2 star HD 22915.

All images were flat fielded and dark subtracted using the ESO software ECLIPSE (Devillard 2001). A bad pixel mask calculated from the sky flat as well as a $3 \sigma$ filtering algorithm were used to clean the images from bad or hot pixels. In order to co-add all images in just a few steps we used the TERAPIX ${ }^{1}$ (Bertin et al. 2002) software SCAMP (Bertin 2006). The calculations of SCAMP are based on catalogs produced by Source Extractor (SE, Bertin \& Arnout

\footnotetext{
1 Traitement elmentaire, Reduction et Analyse des PIXels de megacam

2 Software for Calibrating AstroMetry and Photometry
} 


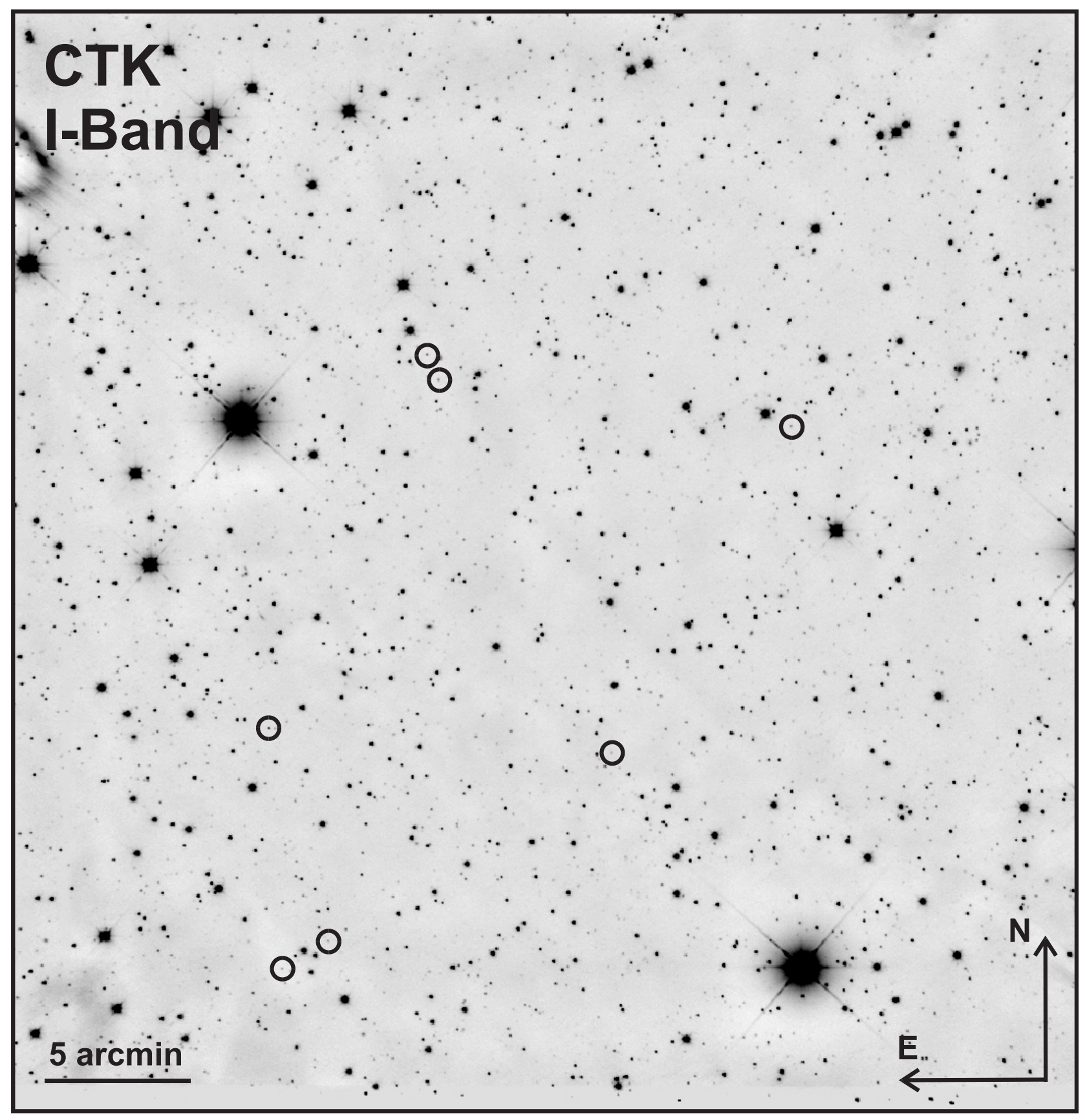

Abbildung 2 The summed up $I$ Filter image with a total exposure time of $47 \mathrm{ks}$ is shown. The center of the field is $\alpha=3^{h} 42^{m} 20.6^{s}, \delta=+25^{\circ} 36^{\prime} 54^{\prime \prime}$. North is up east is left. The brown dwarf candidates we have detected are marked by circles. For more details see Fig. 1 1 and Fig. 10 to Fig. 14

1996). The $S E$ uses thresholding and deblending $\mathrm{g}^{3}$ for source detection, which is in fact more convenient for galaxies than for stars. However the limiting factor for the astrometric accuracy is undersampling, given the CTK pixel scale. Using such object catalogs for each image and the 2MASS catalog (Cutrie et al. 2003) obtained from ViZier (Ochsenbein et al. 2000) as astrometric reference SCAMP computes astrome-

\footnotetext{
${ }^{3}$ For a detailed description of the source detection algorithm used by
} SE see Bertin \& Arnout (1996) and references therein. tric solutions using a $\chi^{2}$ algorithm and applies a polynomial based field distortion correction, involving distortion coefficients up to an order of 10 .

We calculated the astrometric solution for each image and therefore the field distortion pattern of the CTK with a polynomial of the fifth order, shown in Fig. 3 . Given those distortion corrections we co-added all images of each filter using the software SWarp (Bertin et al. 2002). Subtracting all objects, detected by the SE SWarp applies a mesh based 

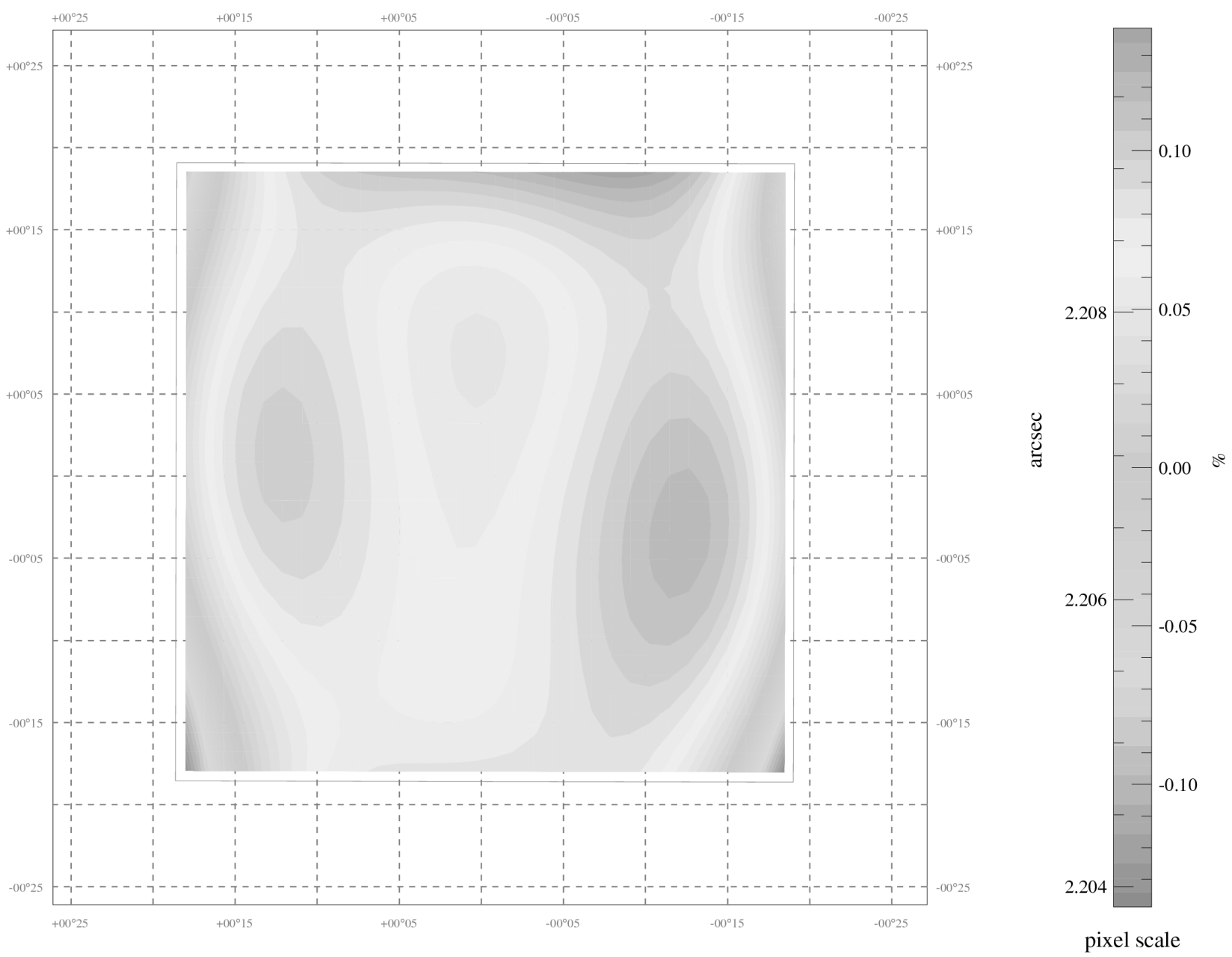

Abbildung 3 Field distortion map of the CTK detector in October 2008. This is originally a colored image. Color coding goes from red (dark spots in the image and top of the scale) through yellow and green (light spot in the middle and vertical light stripes) to blue (corners of the image and bottom of the scale.) The pixel scale variations are given at the color bar, left in arcsec/pixel, right in \%. The difference between the center and the edge is about $0.2 \%$, hence, less than 5 mas.

background algorithm to estimate the background. Since we got small scale illumination effects in the image, produced by bright nearby stars outside the field we used a mesh size of just 32 pixel to overcome these effects. Nevertheless the region around saturated stars is excluded from analysis anyway, since the background flux is overestimated around these regions, see Fig. 1 and Fig. 2

\section{Photometry}

We tried different photometry approaches. We achieved best results, hence reasonable errors and an accurate detection of faint objects, by using the source extractor for the source detection and ESO MIDAS s aperture photometry program (see e.g. Banse et al. 1987) for obtaining the magnitudes. The SE uses thresholding and deblending to distinguish between objects and background and to disentangle close objects whose illumination patterns are mixed. In that way also faintest objects are detectable.
We determined instrumental magnitudes for all sources in the co-added image found by SE using aperture photometry with the MIDAS command magnitude/circle; the aperture radius used in our image for the source photons is 4.5 pixels, the background was measured in a ring around the source with radii between 15 and 22 pixels.

As reference for photometric calibration we have used the catalog of Deacon \& Hamply (2004a) published in Deacon \& Hamply (2004b). They measured $R I$ photometry and proper motion of stars in this region of the Pleiades cluster from Schmidt plates and calculated membership probabilities. In total 15 stars of our field where available. These stars are partly known as flare stars and the accuracy of the Schmidt plates photometry decreases for bright objects. So we excluded 4 of the 15 stars and used the remaining 11 (given in Tbl. 2) to calibrate our images. The zero point was defined as difference between our instrumental magnitudes and that reported by Deacon \& Hamply (2004a). Taking the mean of these differences of all 11 
Tabelle 2 Known Pleiades in the investigated field with known $R$ and $I$ band photometry and with membership probability larger than 0.8 (Deacon \& Hamply 2004a). (There is also a known Pleiades brown dwarf at $\alpha=03: 41: 41$, $\delta=+25: 54: 23$ which happens to lie a few arcseconds outside our field.)

\begin{tabular}{lccccc}
\hline \hline Id & $\alpha(\mathbf{J} 2000)$ & $\delta(\mathrm{J} 2000)$ & Rmag & Imag & prop. \\
\hline $\mathrm{Cl}^{*}$ Melotte 22 HHJ 270 & 3428.270 & 25370.06 & 16.831 & 14.985 & 0.875 \\
$\mathrm{Cl}^{*}$ Melotte 22 SRS 82788 & 3424.850 & 253948.01 & 14.716 & 13.326 & 0.895 \\
$\mathrm{Cl}^{*}$ Melotte 22 SRS 79717 & 34243.800 & 25326.09 & 15.847 & 14.479 & 0.905 \\
$\mathrm{Cl}^{*}$ Melotte 22 HCG 55 & 34154.200 & 254347.08 & 15.893 & 14.444 & 0.923 \\
$\mathrm{Cl}^{*}$ Melotte 22 HCG 96 & 3437.560 & 253429.07 & 16.763 & 14.908 & 0.955 \\
$\mathrm{Cl}^{*}$ Melotte 22 MSH 9 & 3423.410 & 252239.08 & 17.235 & 15.442 & 0.890 \\
$\mathrm{Cl}^{*}$ Melotte 22 MBSC 19 & 34334.130 & 253526.06 & 16.072 & 14.454 & 0.957 \\
$\mathrm{Cl}^{*}$ Melotte 22 SK 733 & 34110.260 & 254556.07 & 16.503 & 14.790 & 0.942 \\
$\mathrm{Cl}^{*}$ Melotte 22 MBSC 22 & 34336.660 & 25471.03 & 16.172 & 14.441 & 0.872 \\
$\mathrm{Cl}^{*}$ Melotte 22 SRS 76506 & 34335.200 & 252431.06 & 15.998 & 14.426 & 0.947 \\
$\mathrm{Cl}^{*}$ Melotte 22 SRS 76505 & 34337.310 & 252432.08 & 15.396 & 14.035 & 0.763 \\
\hline
\end{tabular}

Tabelle 1 Observations $\log$

\begin{tabular}{lcc}
\hline \hline Date & $\begin{array}{c}\text { Filter } \\
\text { Bessel }\end{array}$ & $\begin{array}{c}\text { tot. Exposure } \\
{[\mathrm{s}]}\end{array}$ \\
\hline March 11th, 2007 & $R$ & 5400 \\
March 12th, 2007 & $R$ & 5280 \\
March 13th, 2007 & $R$ & 5520 \\
March 14th, 2007 & $R$ & 4380 \\
March 15th, 2007 & $R$ & 4380 \\
Jan 8th, 2008 & $R$ & 3540 \\
Jan 9th, 2008 & $R$ & 1140 \\
Jan 13th, 2008 & $R$ & 2340 \\
March 23rd, 2008 & $R$ & 1800 \\
March 29th, 2008 & $R$ & 1620 \\
\hline Sep 26th, 2008 & $I$ & 6480 \\
Sep 27th, 2008 & $I$ & 720 \\
Sep 28th, 2008 & $I$ & 9120 \\
Sep 29th, 2008 & $I$ & 6960 \\
Oct 7th, 2008 & $I$ & 3540 \\
Oct 12th, 2008 & $I$ & 3060 \\
Oct 23rd, 2008 & $I$ & 11520 \\
Oct 24th, 2008 & $I$ & 1320 \\
Oct 26th, 2008 & $I$ & 3660 \\
\hline
\end{tabular}

stars we get zero points of $C_{R}=(-19.26 \pm 0.26)$ mag and $C_{I}=(-18.52 \pm 0.17)$ mag for the $I$ band image respectively. We give the $1-\sigma$ calibration error $\left(\sigma_{c a l, R}=0.26\right.$, $\left.\sigma_{c a l, I}=0.17\right)$. The typical accuracy of our measurements is $\sigma_{m e s h, R}=0.18$ and $\sigma_{m e s h, I}=0.14$. The individual integration time was 60 s per image. Objects with apparent magnitudes down to $\sim 22 \mathrm{mag}$ in $R$ and $\sim 20 \mathrm{mag}$ in $I$ are detectable in the average of all our CTK images taken in Rand I-band.

\section{Results}

In total we detected 2401 objects in $R$ and $I$ (only objects visible in both, the $R$ and the $I$ image, are studied further). The objects are cross-correlated with each other using a self written procedure originally designed to calculate exact pro- per motions (Eisenbeiss et al. 2007). The objects are detected at a threshold of 1.5 (relative to the sky) and with 64 deblending grades (maximum for the SE). Since there are small scale background fluctuations in the image we applied a mesh based background calculation with a mesh size of 16 pixels only. We obtained $R I$ photometry for known Pleiades members from the WEBDA database (Mermilliod 1998). We used basic $R I$ Johnson photometry for the bright stars and a list of CCD Cousins observations of faint objects, given at WEBDA. We can now plot our objects in a $R-I$ vs. $I$ color magnitude diagram.

To plot the Pleiades main sequence, we can now use data from all known Pleiades members while we were limited to known Pleiades within our FoV for the photometric calibration of the image. Both are comparable to our Bessel filter system. Based on this literature data we fitted the Pleiades by three linear slopes (Fig. 4). The first straight line fits the Pleiades main sequence. The third straight line fits the Pleiades brown dwarf locus and the second straight line interpolates in between these regions. This approximation represents the Pleiades main sequence with sufficient accuracy, according to our photometric precision. (Fig. 4).

The photometry error of each source in the co-added $R$ and $I$ image is calculated through the calibration error $\sigma_{\text {cal }}$ (see section 4) and the accuracy of the measurement (random error, see section $40 \sigma_{\text {mesh }}$ as $\sigma_{\text {phot }, \text { col }}^{2}=\sigma_{\text {cal }}^{2}+$ $\sigma_{m e s h}^{2}$. The photometry errors of the $R$ and $I$ measurements for each object define the error of the $R-I$ color term as $\sigma_{p h o t, R-I}^{2}=\sigma_{p h o t, R}^{2}+\sigma_{p h o t, I}^{2}$. In a $I$ vs. $R-I$ diagram the full photometry error is than defined as $\sigma_{p h o t}=$ $\sqrt{\sigma_{\text {phot }, R-I}^{2}+\sigma_{\text {phot }, I}^{2}}$. The total offset $\Delta$ allowed for membership must not exceed the photometry error of the object plus the mean residuals $\sigma_{\text {res }}$ of the fitting $\Delta \leq \sqrt{\sigma_{\text {phot }}^{2}+\sigma_{\text {res }}^{2}} . \sigma_{\text {res }}$ is defined as the mean of the fitting residuals of all sources with respect to the approximating lines. This is illustrated in Fig. 5, or in words: The 2D-distance of one source in the $I$ vs. $R-I$ diagram from the line fitted Pleiades main sequence must not exceed the 


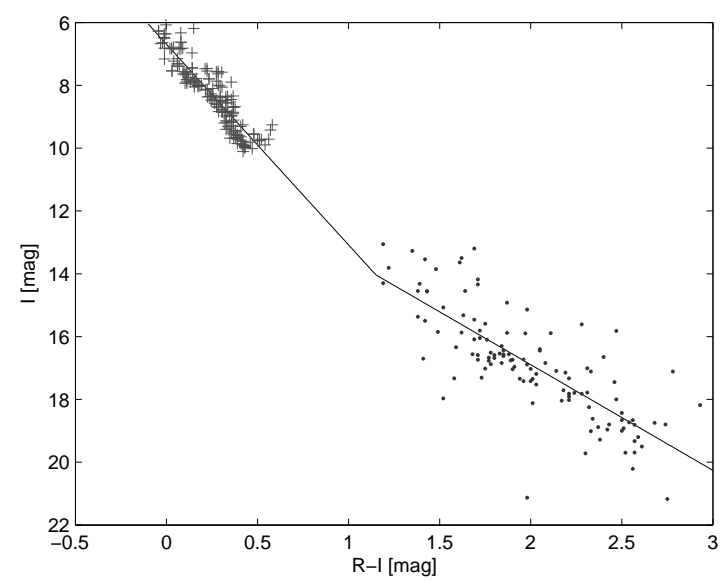

Abbildung 4 Color magnitude diagram $I$ vs. $R-I$. Crosses represent $R I$ Johnson photometry of known Pleiades. Dots represent $R I$ CCD Cousins photometry of known Pleiades low mass stars and brown dwarfs. The black linear slopes are the fits to the data: stellar main sequence is top left, low mass objects is bottom right.

quadratic sum of the photometry error and the fitting residuals.

Additionally each object was checked with the method described above, but under the assumption of unresolved binarity. Such objects would be located slightly above the Pleiades main sequence, but might be additional membership candidates, since the true apparent magnitude of each single object would be higher (up to $0.75 \mathrm{mag}$ if the source was an unresolved equal mass binary). Such objects are marked with five-pointed stars in Fig. 5 .

This way we detected 197 objects with $R I$ photometry consistent with the Pleiades.

For comparison we plotted our data with theoretical isochrones. We used the distance module given in An et al. (2007) of $5.66 \mathrm{mag}$ and plotted our Pleiades membership candidates together with one theoretical sets of isochrones Baraffe et al. (2002), see Fig. 6 The Baraffe models should have a good treatment of the M-dwarfs and brown dwarfs, but our objects are lying above these isochrones. Given the uncertain distance of each object, the limited photometric accuracy of our data and the theoretical difficulties in the modeling of cool dwarfs (dusty atmosphere) the agreement is acceptable.

Then we cross checked our object list with the "Two micron all sky survey" catalog of point sources (2MASS, Skrutskie et al. 2006). If our sources are listed in this cata$\log$ the corresponding identifier is given in the tables. Again we used our self written software to associate the 2MASS sources with ours. We gave an initial search radius of $1 \operatorname{arcsec} \times$ epoch difference ( $\sim 10 \mathrm{yr})$, but the software selects always the closest source as reference if there is more than one source in the search radius. This way we avoid to miss objects, but mismatching can occur if there is no sour-

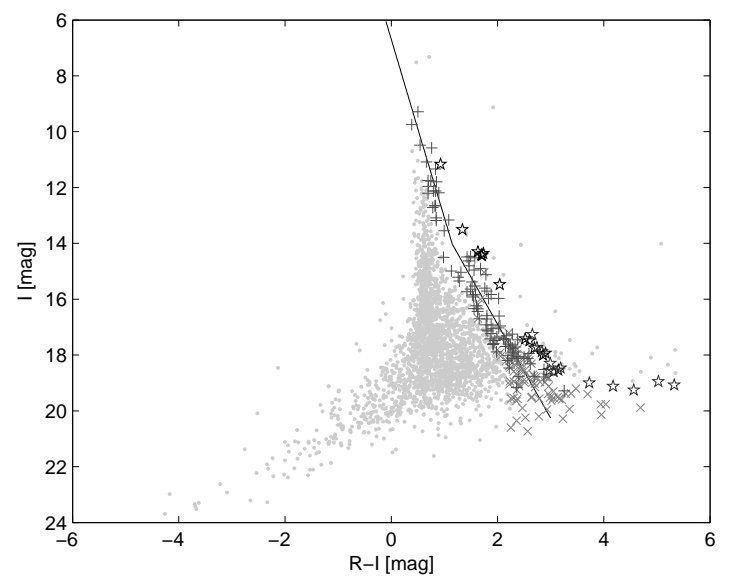

Abbildung 5 The light gray dots are the detected background stars in our observed field. dark gray pluses are the candidate members of the Pleiades with associated 2MASS photometry. The crosses denote the Pleiades membership candidates detected by us, but not by the Two Micron All Sky Survey. The five-pointed stars denote objects, which might be Pleiades candidates under the assumption that these sources are unresolved binaries.

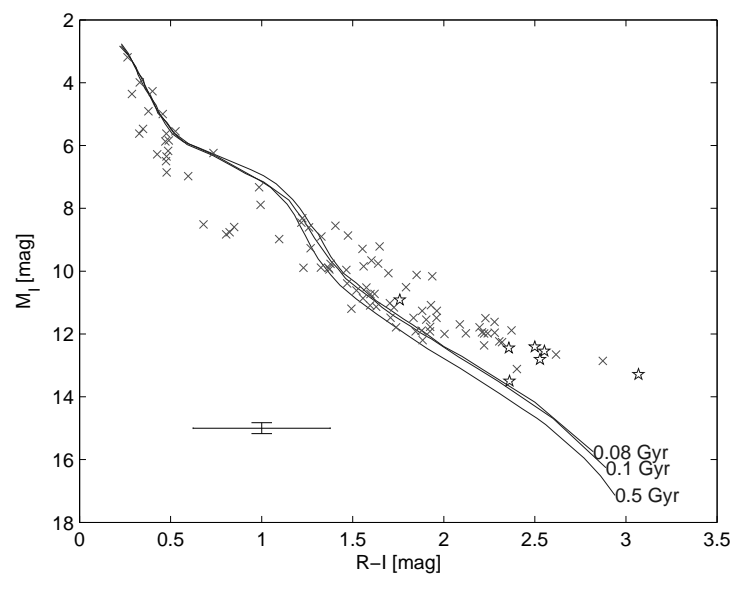

Abbildung 6 Comparison of the candidate members of the Pleiades (x) with three sets of theoretical isochrones. The lines are the Baraffe isochrones from 1998 (Baraffe et al. 2002) for 0.08, 0.1, and 0.5 Gyr. A distance module of $5.66 \mathrm{mag}$ as reported by An et al. (2007) is applied. The agreement is acceptable given the uncertainties of the data and the models. The ages of the isochrones selected according to the reported age of the Pleiades of $\sim 120$ Myr. The brown dwarf candidates found in this study are marked with five-pointed stars. For further explanations see text. 
ce in our images at the position of the 2MASS source. Since we are interested in the faintest 2MASS objects we did not exclude any source by having bad photometry flags. If the object was not found in the 2MASS catalog we named it GSHCTK (for Großschwabhausen-Cassegrain TeleskopKamera) and coordinates.

1244 of all sources (Pleiades candidates and others) are detected by the 2MASS. We estimated the spectral type (SpT) and the interstellar extinction $\left(A_{V}\right)$ for the sources, found in the 2MASS catalog. For these objects enough color information is available. We downloaded an interstellar extinction map from the NAS/IPAC infrared science archive website ${ }^{4}$ and estimated the expected extinction in the visual to be $A_{V} \approx 0.5 \ldots 1$ (see Fig. 77). Other color correction terms were calculated, applying the formulas given in Rieke $\&$ Lebofsky (1985). The ratio of total to selective extinction is $R_{V}=A_{V} / E_{B-V}=3.1$. For reference for the $\mathrm{SpT}$ we used the table given in Kenyon \& Hartmann (1995) in the range from B0 to M6 and from M6.5 to L5 we used the reference stars collected at web page of Neill Rei ${ }^{5}$

Given these data we performed a $\chi^{2}$ minimization for each object with reference to the given information. Within the SpT vs. Av plane a global minimum in $\chi^{2}$ should indicate the most probable color at the most probable $A_{V}$. An example for the visualisation is given in Fig. 8 Given an assumed extinction the $\mathrm{SpT}$ of the object is determined by fitting the data in Kenyon \& Hartmann (1995) or the data of Reid, assuming that all objects are main sequence stars (i.e. luminosity class $\mathrm{V}$ dwarfs). This is repeated for a range of $A_{V}$ creating a 2- dimensional grid of $\chi^{2}$. The global minimum of this grid indicates the best $\mathrm{SpT}$ and $A_{V}$. The error bars of the photometry are taken into account during the minimization of $\chi^{2}$. The results of that effort are summarized in Tbl. A1. With the same method we studied also objects which are presumably not members of the Pleiades, but happen to lie within our FoV and have 2MASS counterparts. Their photometry and spectral type is given in Tbl. A2. To estimate the uncertainties of our resulting quantities (SpT and $A_{V}$ ) we normalized the $\chi^{2}$ matrix. The $1-\sigma$ error range for the normalized $\chi^{2}$ of a 2-dimensional fit is \pm 1 . Every SpT/ $A_{V}$ combination having a normalized $\chi^{2}$ within this interval can be a valid result and determines the uncertainty of SpT and $A_{V}$ as given in the brackets in the corresponding rows of Tbl. A1 and A2. In case of poor photometry it happens, that the $\chi^{2}$ minimization results in more than one (typically 2) local minima. Typically one of these minima is located at low extinction and a late spectral type, while the other minimum can be found at a high value of extinction with an early spectral type. We tried to minimize that effect by presetting the possible range of $A_{V}=0 \ldots 2$ (objects with dusty envelopes or distant galaxies are probably not well determined). However in this cases a wide range of possible spectral types and $A_{V} \mathrm{~s}$ occurs in the tables and the $\chi^{2}$ is typically large, indicating that the fitting

\footnotetext{
4 http://irsa.ipac.caltech.edu/applications/DUST/

5 http://www-int.stsci.edu/ inr/intrins.html
}

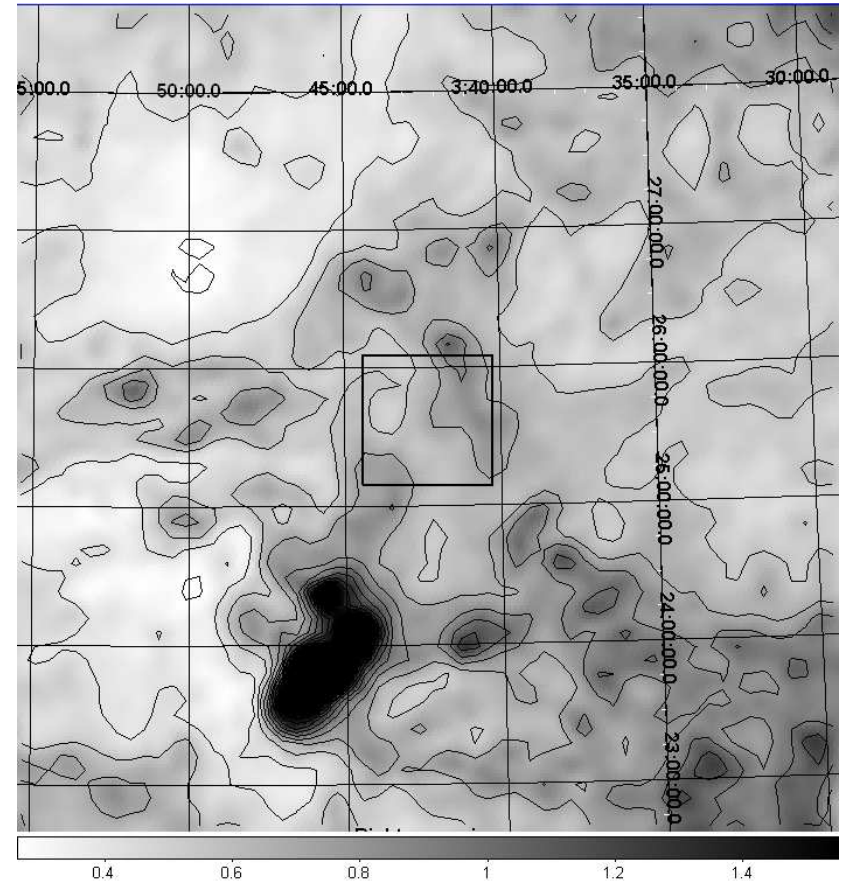

Abbildung 7 Extinction map of the Pleiades region, obtained from the NAS/IPAC infrared science archive. Our FoV is marked by a black box. The interstellar extinction in the V-Filter $\left(A_{V}\right)$ is color coded. One can clearly see that our observations are taken in a region of low extinction.

results are not unique. Finally the objects without near infrared (NIR) counterpart (with more Pleiades membership candidates among them) are listed in Tbl. A3. The table is divided by horizontal lines to indicate again brown dwarf candidates, stellar Pleiades membership candidates and all other stars in that order.

Out of the 197 candidate members 104 are found in the 2MASS catalog. Although these are the bright objects 7 of them are brown dwarf candidates. The individual objects are shown in Fig. 10 to Fig. 14. The border between BDs and LMSs was defined according to the most massive BD known in the Pleiades, Teide 2 (Martín \& Basri 1998), which has a spectral type of M6.5, a mass of $0.072 M_{\odot}$ and $I_{c}=17.82 \mathrm{mag}$ and is considered to be an object on the LMS/BD border. The membership candidates are listed in Tbl. A1 (BD candidates first).

Fig. 9 shows the candidate members of the Pleiades in the $I-K$ vs. $I$ color magnitude diagram, together with the Baraffe isochrones already mentioned above and shown in Fig. 6 in the $R-I$ vs. $I$ plane. The situation changed between the two images. Although in both images extinction corrected magnitudes are shown some objects do not fit the Baraffe isochrones. These objects do not have a fully consistent set of color information. The photometry of this sources is poor in some cases (see Tbl. A1), for others the applied distance module is wrong since these are not Pleiades members. Furthermore the modeling might be inaccurate since it is still difficult to calculate the evolution of cool stars. 

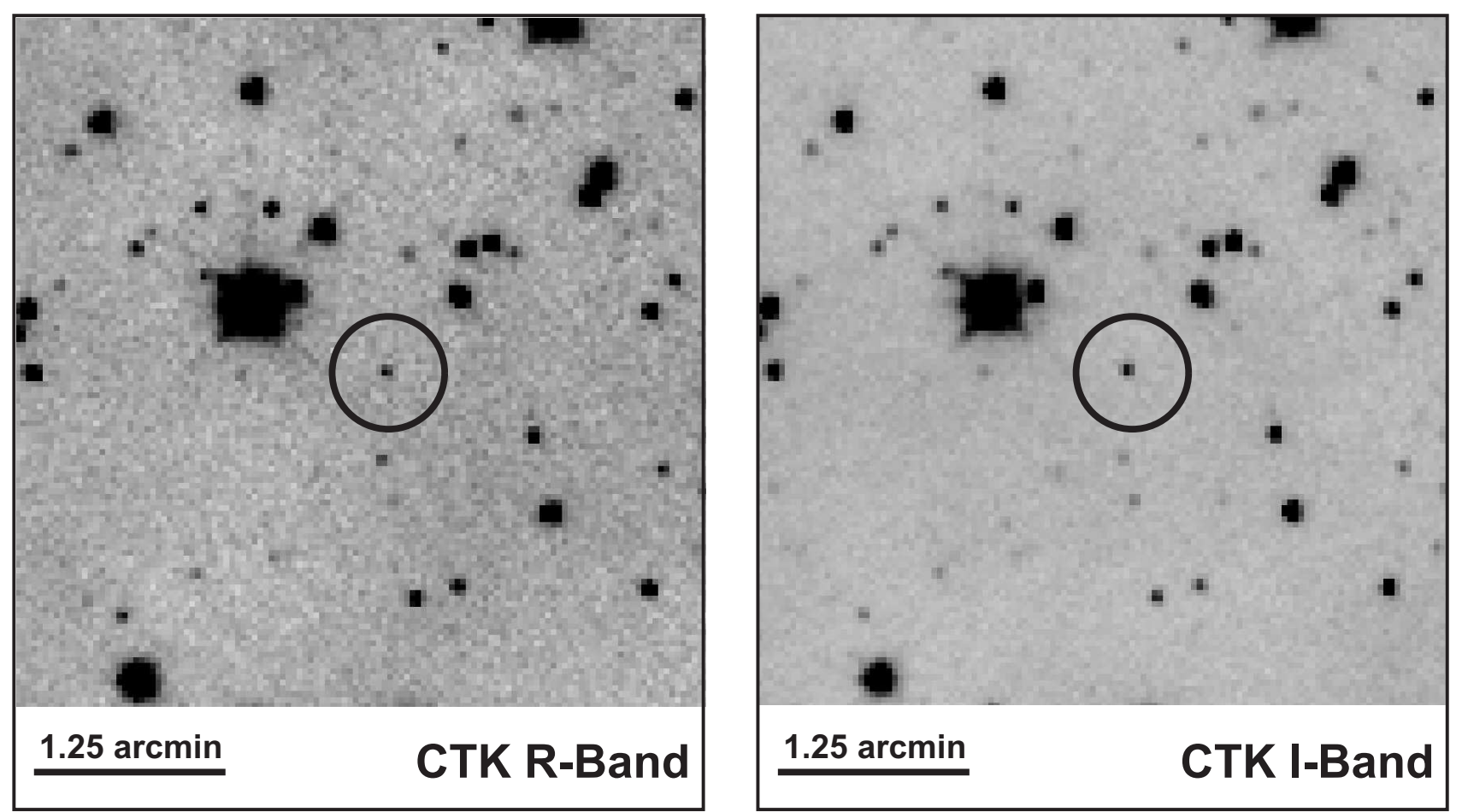

Abbildung $10 \quad$ 2MASS J3414296+2540432
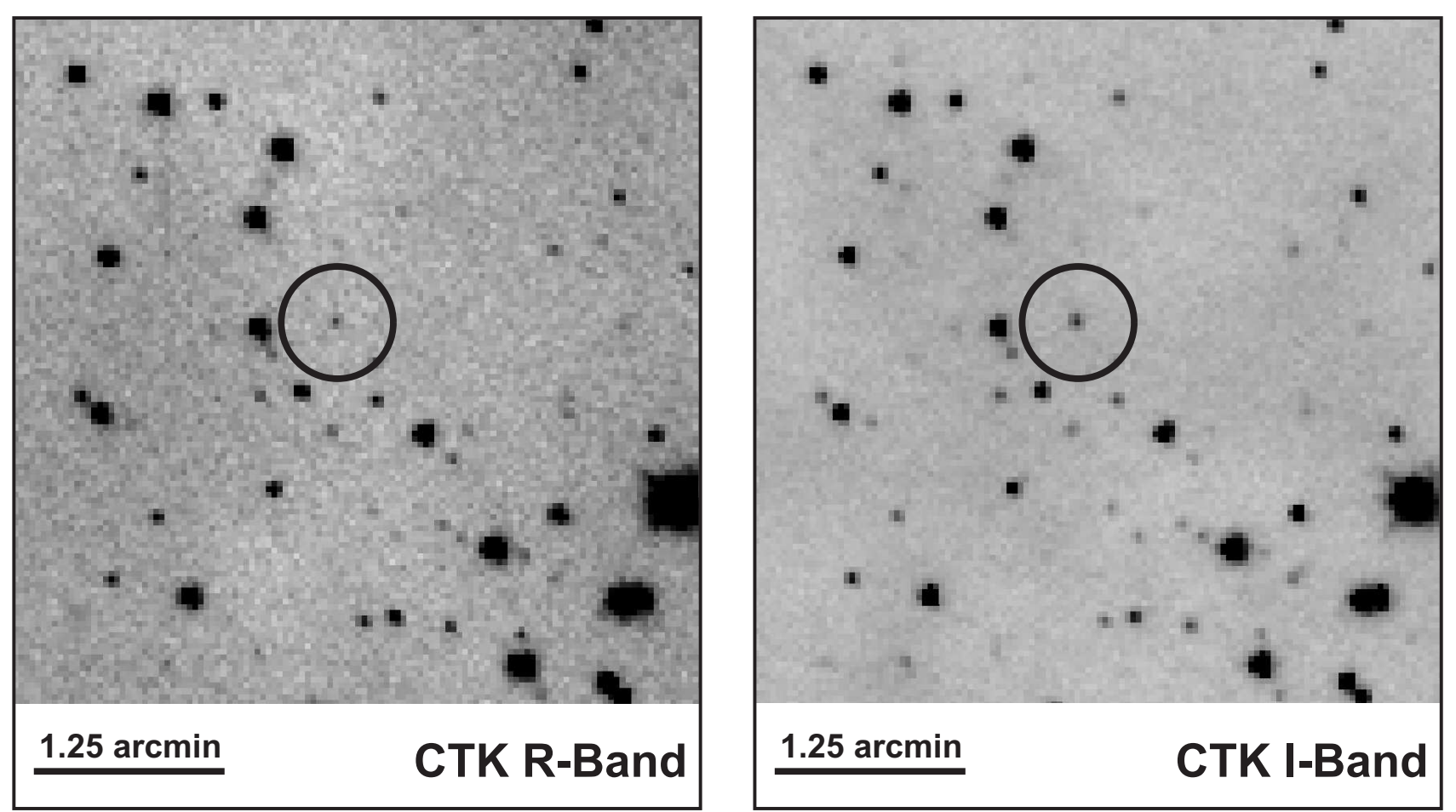

Abbildung 11 2MASS J3421030+2529316 

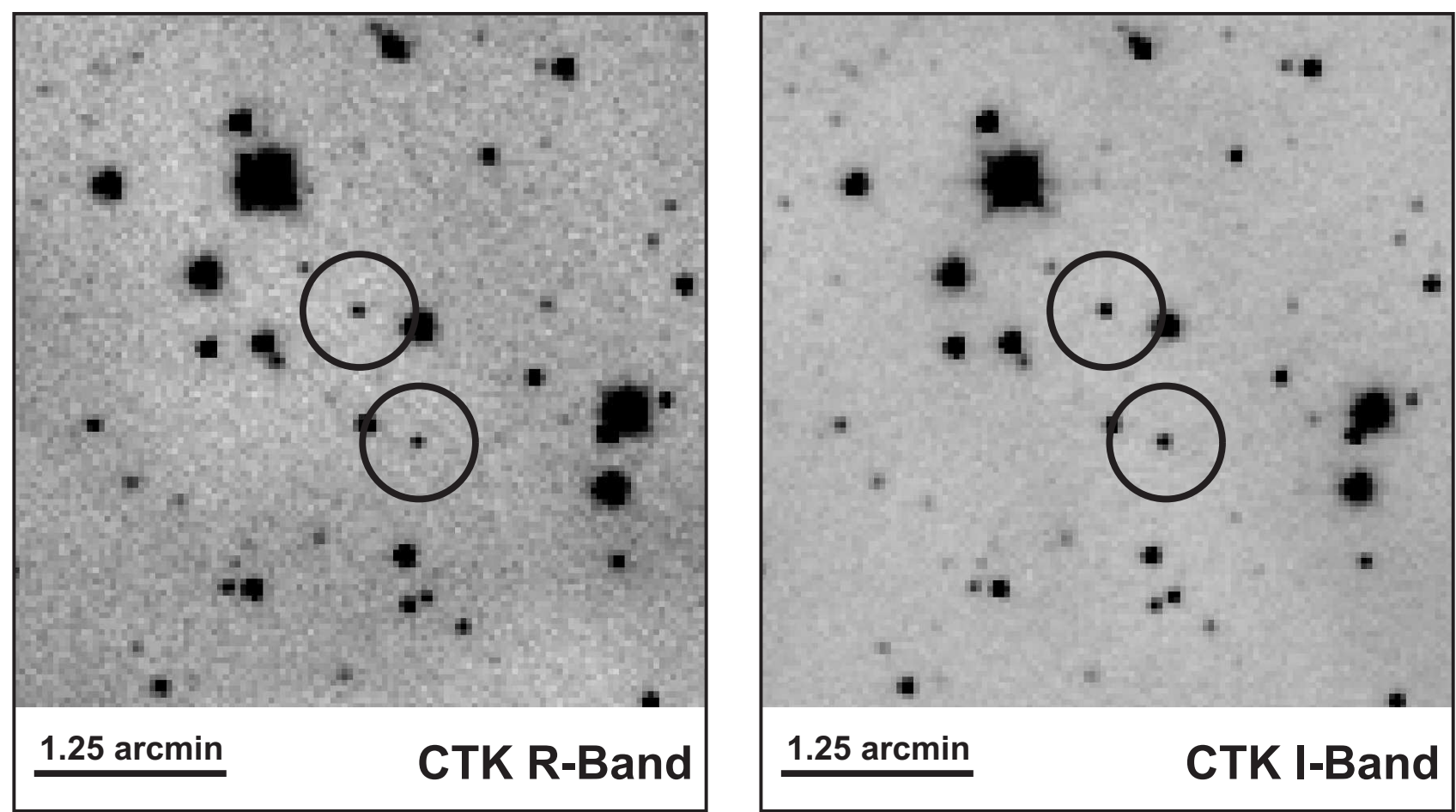

Abbildung 12 2MASS J3423655+2542193 (lower) and 2MASS J3423828+2543104 (upper)
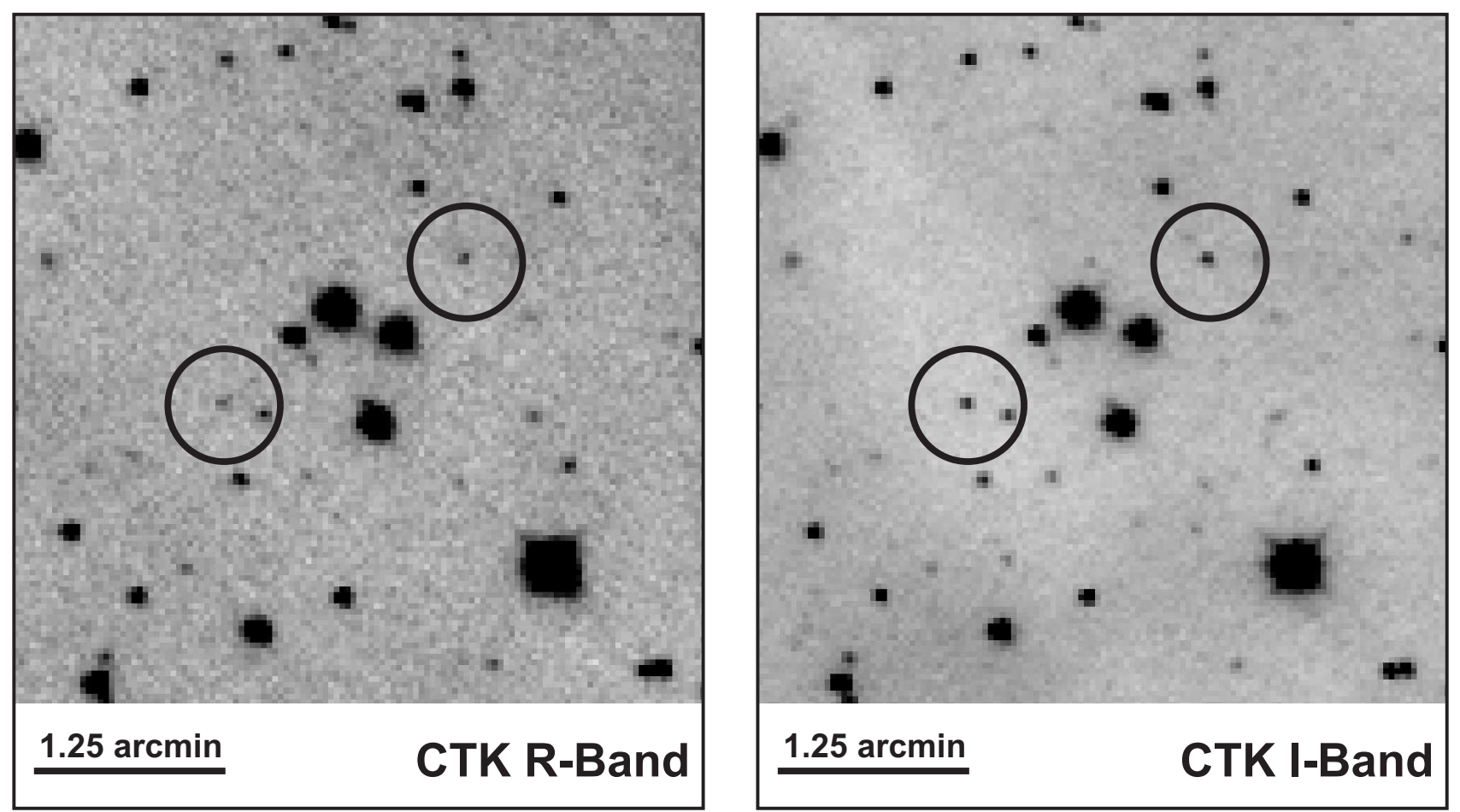

Abbildung 13 2MASS J3425334+2523044 (upper) and 2MASS J3430027+2522082 (lower) 


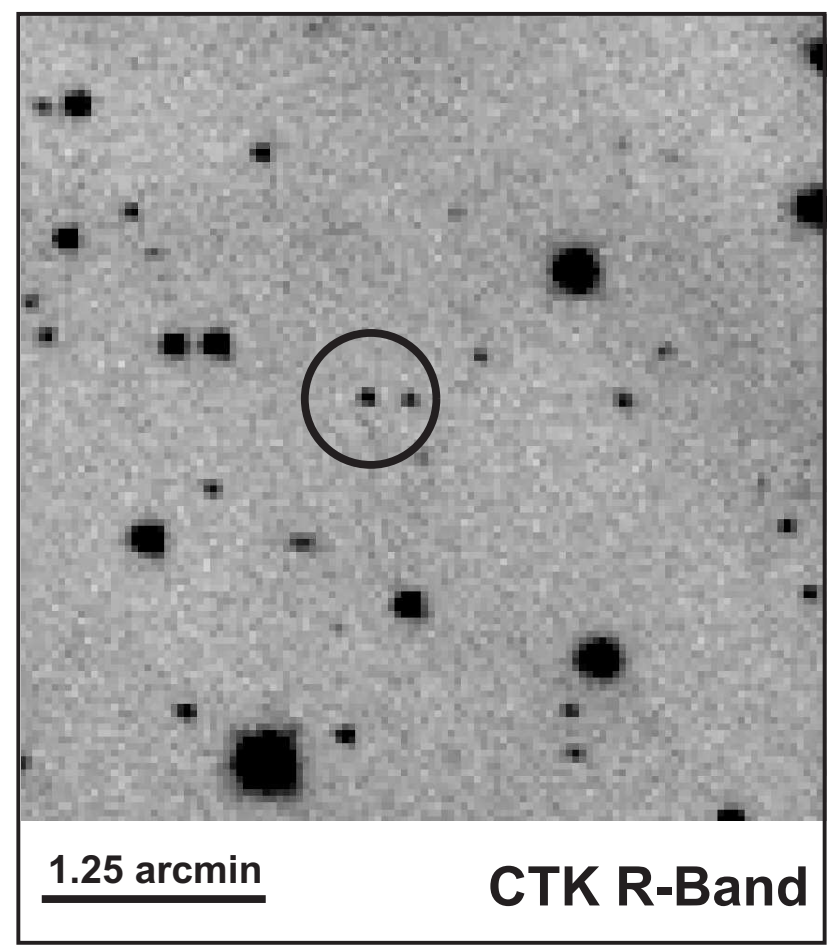

Abbildung 14 2MASS J3430237+2530225

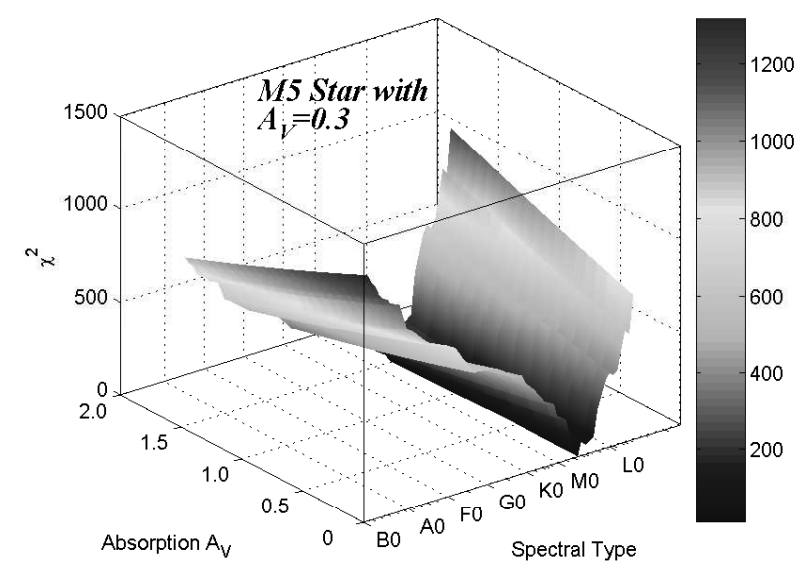

Abbildung 8 Example illustrating in which way we estimated the spectral type and extinction according to the $R I J H K_{s}$ photometry. For an assumed extinction the $\chi^{2}$ minimization is used to fit the data to the corresponding spectral type, defined by the same colors by tables of Kenyon \& Hartmann (1995) and Reid, N. The extinction is varied within a reasonable range $\left(A_{V}\right.$ between 0 and 2 in this case) and a 2-dimensional grid of $\chi^{2}$ is created. The global minimum of this grid gives the extinction and the spectral types. In the tables A1 and A2 the uncertainties of the spectral type and the extinction according to the $1-\sigma$ error of $\chi^{2}$ is given.
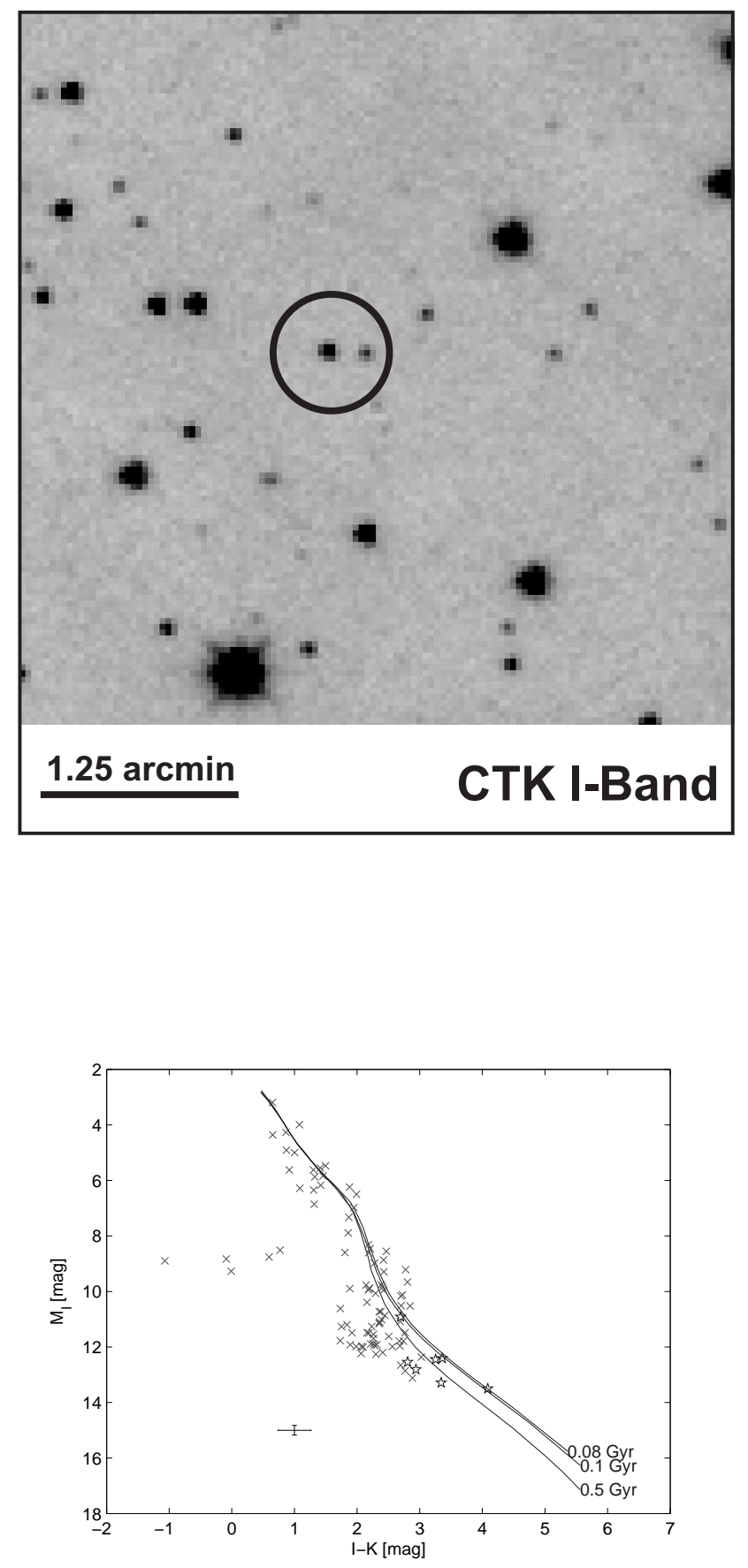

Abbildung 9 Comparison of the candidate members of the Pleiades (x) with Baraffe theoretical isochrones (Baraffe et al. 2002). A distance module of 5.66 as reported by An et al. (2007) is applied. Ages the of Isochrones are given in the figure and selected according to the reported age of the Pleiades of $\sim 120$ Myr. In contrast to Fig. 6 in the $I-K$ vs. I plane the data of the brown dwarf candidates (stars) are in good agreement with the Baraffe models while some other sources are not fitted at all by the isochrones. This indicates poor photometry for these objects or the assumption of Pleiades membership is wrong. For further explanations see text. 


\section{Summary and Conclusions}

We have obtained deep imaging observations of one specific Pleiades field, located at the rim of the Pleiades cluster with a size of $\sim 38^{\prime} \times 38^{\prime}$. We have detected objects down to $R \sim 22 \mathrm{mag}$ and $I \sim 20 \mathrm{mag}$. Among those objects 104 Pleiades candidates could be studied further since 2MASS photometry was available. This study identified 7 very red candidate members of the Pleiades. If these objects have Pleiades age and distance they could be massive brown dwarfs. This has to be ensured by spectroscopy and proper motion analysis, both not possible with the instrument used for this study.

At the edge of a stellar cluster the member density is low. Such studies can give hints for BD formation theories. If BDs form like normal stars and high mass stars are formed in the center of molecular clouds it can be considered that more LMS and BD form further outside at the edges of clouds, where the material density is lower. Also BDs and LMS formed near the cluster center may get ejected and are now located near the edge or outside the cluster. The frequency of BDs at the cluster edge should be different from the cluster center (see Kroupa \& Bouvier, 2003 and Kroupa et al. 2003 for a detailed discussion on this argument).

Further investigations are needed to confirm or reject the suspected BD candidates. Therefore a proper motion analysis and more precise photometry as well as spectroscopy is necessary. In particular NIR photometry of the other suspected membership candidates (not detected by 2MASS) is needed.

Acknowledgements. TE would like to thank Katharina Schreyer for her help and advise. RN acknowledges general support from the German National Science Foundation (Deutsche Forschungsgemeinschaft, DFG) in grants NE 515/13-1, 13-2, and 23-1, AK acknowledges support from DFG in grant KR 2164/8-1, SR and MV acknowledge support from the EU in the FP6 MC ToK project MTKD-CT-2006-042514, TOBS acknowledges support from the Evangelisches Studienwerk e.V. Villigst, TR acknowledges support from DFG in grant NE 515/23-1, TE and MH acknowledge partial support from DFG in the SFB TR-7 Gravitation Wave Astronomy. M. Moualla acknowledges support from the Syrian government. This research has made use of the VizieR catalogue access tool and the Simbad database, both operated at the Observatoire Strasbourg, as well as of the WEBDA database, operated at the Institute for Astronomy of the University of Vienna and data products from the Two Micron All Sky Survey, which is a joint project of the University of Massachusetts and the Infrared Processing and Analysis Center/California Institute of Technology, funded by the National Aeronautics and Space Administration and the National Science Foundation, and of the NASA/ IPAC Infrared Science Archive, which is operated by the Jet Propulsion Laboratory, California Institute of Technology, under contract with the National Aeronautics and Space Administration.

\section{Literatur}

An, D., Terndrup, D.M., Pinsonneault, M.H., et al 2007, ApJ 655, 233
Banse, K., Ounnas, C., Ponz, D., Grosbol, P., \& Warmels, R. 1987, BA\&AS 19, 738

Baraffe, I., Chabrier, G., Allard, F., \& Hauschildt, P. H. 2002, A\&A 382, 563

Bertin, E., \& Arnouts, S. 1996, A\&APS 117, 393

Basri, G., Marcy, G., \& Graham, J.R. 1996, ApJ 458, 600 (BMG)

Bertin, E., Mellier, Y., Radovich, M., et al. 2002 ASPC 281, 228

Bertin, E. 2006, ASPC 351, 112

Bouvier, J.R., Stauffer, E.L., Martín, D., et al. 1998, A\&A 336, 490

Cutri, R.M., Skrutskie, M.F., van Dyk, S., et al. 2003, 2MASS All Sky Catalog of point sources. (The IRSA 2MASS AllSky Point Source Catalog, NASA/IPAC Infrared Science Archive. http://irsa.ipac.caltech.edu/applications/Gator/)

Deacon, N.R., \& Hamply, N.C. 2004, VizieR On-line Data Catalog $\mathrm{J} / \mathrm{A}+\mathrm{A} / 416 / 125$

Deacon, N.R., \& Hamply, N.C. 2004, A\&A 416, 125

Devillard, N. 2001, Astronomical Data Analysis Software and Systems X, 238, 525

Eisenbeiss, T., Seifahrt, A., Mugrauer, M., et al. 2007, AN 328, 521

Kenyon, S. J., \& Hartmann, L. 1995, ApJ 101, 117

Kroupa, P., \& Bouvier, J. 2003, MNRAS 346, 369

Kroupa, P., Bouvier, J., Duchêne, G., \& Moraux, E. 2003, MNRAS 346, 354

Martín, D., Dahm, S.: 2001, ASPC 245, 349

Martín, E.L., \& Basri, G. 1998, ApJ 499, 64

Mermilliod, J.-C. ed. 1998, WEB Acces to the Open Cluster Database

Moraux, E., Bouvier, J.R., Stauffer, E.L., et al. 2003, A\&A 400, 891

Moualla, M., Raetz, S., Neuhäuser, R., et al.: in prep.

Mugrauer, M. 2009, AN, this issue

Ochsenbein, F., Bauer, P., \& Marcout, J. 2000, A\&As 143, 23

Rebollo, R., Martín, E.L., Basri, G., et al. 1996, ApJS 469, L53

Rieke, G. H., \& Lebofsky, M. J. 1985, ApJ 288, 618

Schwartz, M.J., \& Becklin, E.E. 2005, AJ 130, 2352

Skrutskie, M. F., et al. 2006, AJ 131, 1163 
A Lists of objects 
Tabelle A1 Extinction corrected colors and spectral type for the new Pleiades membership candidates found in this study. Most columns are self explaining. The minimum $\chi^{2}$ value for the estimated spectral type and interstellar extinction is given and can be seen as a quality factor. Objects above the first horizontal line have spectral types later or approximately M6.5 and so are brown dwarf candidates if they are Pleiades members. Objects below the second horizontal line (at the end of the table) are sources located above the Pleiades main sequence in the color magnitude diagram. It is possible that these objects are unresolved binaries and they appear brighter in the sky (about 0.75 magnitudes in the worst case of equal mass binaries). Taking this into account these sources should be mentioned as additional candidate members of the Pleiades but the binary assumption has no observational evidence and the hypothesis of background giants can not be ruled out.

\begin{tabular}{|c|c|c|c|c|c|c|c|c|c|}
\hline \multirow[b]{2}{*}{ 2MASS-ID } & \multicolumn{2}{|c|}{ measured magnitudes } & \multicolumn{4}{|c|}{ extinction corrected colors } & \multirow[b]{2}{*}{$\chi^{2}$} & \multirow[b]{2}{*}{$\mathrm{SpT}$} & \multirow[b]{2}{*}{ Av } \\
\hline & Rmag & Imag & R-I & I-J & $\mathrm{I}-\mathrm{H}$ & $\mathrm{I}-\mathrm{K}_{s}$ & & & \\
\hline $\mathrm{J} 3414296+2540432$ & $21.52 \pm 0.88$ & $19.16 \pm 0.23$ & $2.36 \pm 0.91$ & $3.00 \pm 0.25$ & $3.58 \pm 0.25$ & $4.09 \pm 0.26$ & 1.5 & M8 (M7.5..M8) & $0.00(0.00 . .1 .34)$ \\
\hline $\mathrm{J} 3421030+2529316$ & $21.45 \pm 0.77$ & $18.76 \pm 0.20$ & $2.53 \pm 0.79$ & $2.23 \pm 0.23$ & $2.29 \pm 0.29$ & $2.94 \pm 0.27$ & 4.2 & M6 (M5..M7) & $0.61(0.01 . .1 .40)$ \\
\hline $\mathrm{J} 3423655+2542193$ & $20.49 \pm 0.39$ & $18.13 \pm 0.18$ & $2.36 \pm 0.43$ & $2.28 \pm 0.20$ & $3.17 \pm 0.21$ & $3.25 \pm 0.21$ & 3.1 & M6.5 (M6..M7) & $0.04(0.04 . .1 .24)$ \\
\hline $\mathrm{J} 3423828+2543104$ & $20.57 \pm 0.41$ & $18.07 \pm 0.18$ & $2.50 \pm 0.44$ & $2.55 \pm 0.19$ & $3.13 \pm 0.20$ & $3.36 \pm 0.20$ & 1.7 & M7.5 (M6.5..M7.5) & $0.00(0.00 . .0 .58)$ \\
\hline $\mathrm{J} 3425334+2523044$ & $22.54 \pm 1.82$ & $19.28 \pm 0.25$ & $3.07 \pm 1.83$ & $2.68 \pm 0.28$ & $2.03 \pm$ & $3.34 \pm 0.32$ & 1.7 & M7.5 (M6.5..M7.5) & $0.69(0.05 . .1 .45)$ \\
\hline $\mathrm{J} 3430027+2522082$ & $21.66 \pm 0.87$ & $18.79 \pm 0.22$ & $2.55 \pm 0.90$ & $2.10 \pm 0.24$ & $2.59 \pm 0.27$ & $2.81 \pm 0.30$ & 2.1 & M6 (M6..M7) & $1.20(0.08 . .1 .41)$ \\
\hline $\mathrm{J} 3430237+2530225$ & $19.47 \pm 0.28$ & $17.31 \pm 0.17$ & $1.76 \pm 0.33$ & $1.97 \pm 0.18$ & $2.48 \pm 0.18$ & $2.70 \pm 0.18$ & 3.0 & M5 (M5..M6.5) & $1.53(0.03 . .1 .53)$ \\
\hline $\mathrm{J} 3410382+2532036$ & $18.96 \pm 0.32$ & $17.14 \pm 0.17$ & $1.63 \pm 0.36$ & $1.53 \pm 0.18$ & $2.16 \pm 0.18$ & $2.36 \pm 0.18$ & 1.1 & M4 (M3..M4) & $0.72(0.48 . .1 .40)$ \\
\hline $\mathrm{J} 3410561+2552324$ & $21.39 \pm 0.71$ & $18.52 \pm 0.20$ & $2.87 \pm 0.73$ & $1.64 \pm 0.25$ & $2.16 \pm 0.30$ & $2.77 \pm 0.28$ & 6.9 & M6 (M3...M6) & $0.00(0.00 . .1 .38)$ \\
\hline $\mathrm{J} 3410625+2539555$ & $18.36 \pm 0.27$ & $16.70 \pm 0.17$ & $1.56 \pm 0.32$ & $1.57 \pm 0.18$ & $2.14 \pm 0.18$ & $2.44 \pm 0.18$ & 1.3 & M4 (M3..M4) & $0.38(0.19 . .1 .31)$ \\
\hline $\mathrm{J} 3410780+2524338$ & $17.10 \pm 0.26$ & $15.53 \pm 0.17$ & $1.56 \pm 0.31$ & $1.54 \pm 0.17$ & $2.20 \pm 0.17$ & $2.41 \pm 0.17$ & 1.4 & M4 (M2..M4) & $0.05(0.05 . .1 .48)$ \\
\hline $\mathrm{J} 3411022+2525350$ & $14.03 \pm 0.26$ & $13.18 \pm 0.17$ & $0.48 \pm 0.31$ & $0.79 \pm 0.17$ & $1.25 \pm 0.17$ & $1.32 \pm 0.17$ & 1.3 & K3 (K2..M0) & $1.38(0.12 . .1 .49)$ \\
\hline $\mathrm{J} 3411026+2545562$ & $16.09 \pm 0.26$ & $14.63 \pm 0.17$ & $1.26 \pm 0.31$ & $1.44 \pm 0.17$ & $1.99 \pm 0.17$ & $2.18 \pm 0.17$ & 2.1 & M3 (M2..M4) & $0.75(0.07 . .1 .23)$ \\
\hline $\mathrm{J} 3411301+2553238$ & $19.82 \pm 0.28$ & $17.61 \pm 0.17$ & $2.21 \pm 0.33$ & $0.94 \pm 0.22$ & $1.64 \pm 0.25$ & $2.28 \pm 0.22$ & 17.5 & M4 (M0..M4) & $0.00(0.00 . .1 .38)$ \\
\hline $\mathrm{J} 3411785+2522353$ & $12.66 \pm 0.26$ & $11.96 \pm 0.17$ & $0.33 \pm 0.31$ & $0.62 \pm 0.17$ & $0.86 \pm 0.17$ & $0.92 \pm 0.17$ & 1.9 & G7 (G7..K6) & $1.41(0.03 . .1 .45)$ \\
\hline $\mathrm{J} 3411827+2528311$ & $17.13 \pm 0.26$ & $15.64 \pm 0.17$ & $1.38 \pm 0.31$ & $1.37 \pm 0.17$ & $1.92 \pm 0.17$ & $2.14 \pm 0.17$ & 1.2 & M3 (M2..M3) & $0.43(0.22 . .1 .04)$ \\
\hline $\mathrm{J} 3412068+2531285$ & $12.90 \pm 0.26$ & $12.21 \pm 0.17$ & $0.49 \pm 0.31$ & $0.91 \pm 0.17$ & $1.39 \pm 0.17$ & $1.42 \pm 0.17$ & 1.7 & $\mathrm{~K} 4$ (K2..K6) & $0.77(0.38 . .1 .30)$ \\
\hline $\mathrm{J} 3412129+2520354$ & $19.52 \pm 0.37$ & $17.41 \pm 0.17$ & $2.09 \pm 0.41$ & $1.41 \pm 0.19$ & $1.84 \pm 0.21$ & $2.26 \pm 0.20$ & 6.8 & M4 (M2..M4) & $0.10(0.10 . .1 .46)$ \\
\hline $\mathrm{J} 3412843+2545287$ & $13.94 \pm 0.26$ & $13.09 \pm 0.17$ & $0.60 \pm 0.31$ & $1.19 \pm 0.17$ & $1.84 \pm 0.17$ & $1.95 \pm 0.17$ & 5.4 & M0 (K5..M1) & $0.94(0.29 . .1 .49)$ \\
\hline $\mathrm{J} 3412883+2520161$ & $19.34 \pm 0.31$ & $17.36 \pm 0.17$ & $1.90 \pm 0.35$ & $1.36 \pm 0.18$ & $2.03 \pm 0.19$ & $2.26 \pm 0.20$ & 3.7 & M4 (M2..M4) & $0.31(0.10 . .1 .47)$ \\
\hline $\mathrm{J} 3413143+2525572$ & $12.96 \pm 0.26$ & $12.13 \pm 0.17$ & $0.49 \pm 0.31$ & $0.87 \pm 0.17$ & $1.37 \pm 0.17$ & $1.46 \pm 0.17$ & 1.2 & K4 (K4..M1) & $1.30(0.00 . .1 .46)$ \\
\hline $\mathrm{J} 3413146+2551070$ & $17.16 \pm 0.26$ & $15.73 \pm 0.17$ & $1.32 \pm 0.31$ & $1.33 \pm 0.17$ & $1.96 \pm 0.17$ & $2.20 \pm 0.17$ & 1.1 & M3 (M1..M3) & $0.38(0.23 . .1 .47)$ \\
\hline $\mathrm{J} 3413451+2520479$ & $14.55 \pm 0.26$ & $13.55 \pm 0.17$ & $0.99 \pm 0.31$ & $1.09 \pm 0.17$ & $1.64 \pm 0.17$ & $1.86 \pm 0.17$ & 1.6 & M1 (K3..M1) & $0.00(0.00 . .1 .43)$ \\
\hline $\mathrm{J} 3413516+2546444$ & $20.93 \pm 0.52$ & $18.32 \pm 0.18$ & $2.62 \pm 0.55$ & $1.93 \pm 0.21$ & $2.40 \pm 0.25$ & $2.70 \pm 0.27$ & 3.8 & M6 (M5..M6) & $0.00(0.00 . .1 .24)$ \\
\hline $\mathrm{J} 3413609+2527469$ & $17.98 \pm 0.26$ & $16.35 \pm 0.17$ & $1.47 \pm 0.31$ & $1.25 \pm 0.17$ & $1.85 \pm 0.18$ & $2.16 \pm 0.18$ & 1.5 & M3 (M2..M4) & $0.62(0.01 . .1 .19)$ \\
\hline $\mathrm{J} 3413616+2537095$ & $10.13 \pm 0.26$ & $9.75 \pm 0.17$ & $0.33 \pm 0.31$ & $0.75 \pm 0.17$ & $1.03 \pm 0.17$ & $1.08 \pm 0.17$ & 2.4 & $\mathrm{~K} 0$ (G6..K2) & $0.20(0.02 . .0 .87)$ \\
\hline $\mathrm{J} 3413790+2532178$ & $19.54 \pm 0.29$ & $17.62 \pm 0.17$ & $1.49 \pm 0.34$ & $0.85 \pm 0.21$ & $1.87 \pm \ldots$ & $1.84 \pm \ldots$ & 3.5 & M2 (M1..M3) & $1.59(0.11 . .1 .59)$ \\
\hline $\mathrm{J} 3414143+2550072$ & $18.97 \pm 0.27$ & $17.06 \pm 0.17$ & $1.70 \pm 0.32$ & $1.44 \pm 0.18$ & $2.08 \pm 0.18$ & $2.39 \pm 0.18$ & 1.4 & M4 (M3..M4) & $0.79(0.54 . .1 .42)$ \\
\hline $\mathrm{J} 3414281+2528328$ & $20.39 \pm 0.38$ & $18.12 \pm 0.18$ & $2.22 \pm 0.42$ & $2.13 \pm 0.20$ & $2.57 \pm 0.21$ & $3.03 \pm 0.22$ & 1.9 & M6 (M5..M6) & $0.19(0.02 . .1 .44)$ \\
\hline J3414294+2528126 & $18.06 \pm 0.26$ & $16.44 \pm 0.17$ & $1.60 \pm 0.31$ & $1.50 \pm 0.18$ & $2.23 \pm 0.17$ & $2.35 \pm 0.18$ & 1.7 & M4 (M2..M4) & $0.10(0.01 . .1 .44)$ \\
\hline $\mathrm{J} 3414637+2522220$ & $16.12 \pm 0.26$ & $14.99 \pm 0.17$ & $0.68 \pm 0.31$ & $0.35 \pm 0.17$ & $0.75 \pm 0.17$ & $0.77 \pm 0.17$ & 4.9 & G8 (G5..K7) & $1.69(0.18 . .1 .69)$ \\
\hline $\mathrm{J} 3414903+2526096$ & $17.80 \pm 0.26$ & $15.97 \pm 0.17$ & $1.70 \pm 0.31$ & $1.54 \pm 0.17$ & $2.13 \pm 0.17$ & $2.30 \pm 0.17$ & 1.6 & M4 (M3..M4) & $0.51(0.27 . .1 .34)$ \\
\hline $\mathrm{J} 3414955+2549110$ & $20.69 \pm 0.44$ & $18.47 \pm 0.19$ & $1.88 \pm 0.48$ & $1.31 \pm 0.26$ & $1.95 \pm 0.28$ & $2.41 \pm 0.29$ & 2.6 & M4 (M3..M5) & $1.26(0.08 . .1 .46)$ \\
\hline $\mathrm{J} 3415420+2543473$ & $15.96 \pm 0.26$ & $14.47 \pm 0.17$ & $1.22 \pm 0.31$ & $1.47 \pm 0.17$ & $2.03 \pm 0.17$ & $2.18 \pm 0.17$ & 2.7 & M3 (M2..M4) & $1.02(0.16 . .1 .47)$ \\
\hline $\mathrm{J} 3415606+2527484$ & $19.56 \pm 0.29$ & $17.44 \pm 0.17$ & $1.96 \pm 0.33$ & $1.44 \pm 0.19$ & $1.94 \pm 0.19$ & $2.16 \pm 0.22$ & 5.2 & M4 (M3..M5) & $0.60(0.01 . .1 .15)$ \\
\hline $\mathrm{J} 3415610+2521197$ & $19.84 \pm 0.31$ & $17.69 \pm 0.17$ & $2.12 \pm 0.35$ & $0.89 \pm 0.23$ & $1.34 \pm 0.31$ & $2.07 \pm$ & 13.7 & M3 (K5..M3) & $0.11(0.11 . .1 .50)$ \\
\hline $\mathrm{J} 3420571+2528028$ & $15.92 \pm 0.26$ & $14.49 \pm 0.17$ & $1.22 \pm 0.31$ & $1.48 \pm 0.17$ & $1.99 \pm 0.17$ & $2.21 \pm 0.17$ & 2.8 & M3 (M2..M4) & $0.78(0.00 . .1 .31)$ \\
\hline $\mathrm{J} 3420783+2532278$ & $20.44 \pm 0.41$ & $18.05 \pm 0.18$ & $2.32 \pm 0.44$ & $1.62 \pm 0.21$ & $2.42 \pm 0.21$ & $2.30 \pm 0.27$ & 6.3 & M5 (M3..M6) & $0.27(0.14 . .1 .46)$ \\
\hline $\mathrm{J} 3420828+2537002$ & $16.90 \pm 0.26$ & $15.12 \pm 0.17$ & $1.65 \pm 0.31$ & $1.98 \pm 0.17$ & $2.55 \pm 0.17$ & $2.77 \pm 0.17$ & 4.7 & M5 (M4..M5) & $0.50(0.06 . .1 .42)$ \\
\hline $\mathrm{J} 3420867+2521549$ & $20.39 \pm 0.37$ & $18.20 \pm 0.18$ & $1.85 \pm 0.41$ & $1.45 \pm 0.23$ & $1.98 \pm 0.24$ & $2.31 \pm 0.26$ & 2.1 & M4 (M4..M5) & $1.28(0.03 . .1 .48)$ \\
\hline $\mathrm{J} 3421032+2544298$ & $17.90 \pm 0.26$ & $16.34 \pm 0.17$ & $1.52 \pm 0.31$ & $1.01 \pm 0.18$ & $1.56 \pm 0.18$ & $1.73 \pm 0.19$ & 6.9 & M2 (K6..M2) & $0.14(0.14 . .1 .45)$ \\
\hline $\mathrm{J} 3421098+2544353$ & $17.99 \pm 0.26$ & $15.97 \pm 0.17$ & $1.94 \pm 0.31$ & $1.77 \pm 0.17$ & $2.38 \pm 0.17$ & $2.70 \pm 0.17$ & 1.1 & M5 (M4..M5) & $0.32(0.20 . .1 .46)$ \\
\hline $\mathrm{J} 3421340+2553188$ & $20.70 \pm 0.42$ & $18.13 \pm 0.19$ & $2.20 \pm 0.46$ & $1.10 \pm 0.25$ & $1.96 \pm 0.24$ & $2.67 \pm \ldots$ & 6.7 & M4 (M3..M6) & $1.40(0.04 . .1 .40)$ \\
\hline $\mathrm{J} 3421516+2549517$ & $18.89 \pm 0.27$ & $17.11 \pm 0.17$ & $1.60 \pm 0.32$ & $1.52 \pm 0.18$ & $2.21 \pm 0.18$ & $2.36 \pm 0.19$ & 1.5 & M4 (M3..M4) & $0.73(0.48 . .1 .46)$ \\
\hline $\mathrm{J} 3421553+2536321$ & $20.21 \pm 0.35$ & $18.01 \pm 0.18$ & $2.00 \pm 0.39$ & $1.43 \pm 0.21$ & $1.96 \pm 0.25$ & $2.10 \pm 0.28$ & 4.6 & M4 (M3..M5) & $0.73(0.01 . .1 .39)$ \\
\hline $\mathrm{J} 3422784+2533106$ & $13.50 \pm 0.26$ & $12.71 \pm 0.17$ & $0.48 \pm 0.31$ & $1.31 \pm 0.17$ & $1.88 \pm 0.17$ & $1.99 \pm 0.17$ & 10.1 & M0 (M0..M3) & $1.15(0.22 . .1 .44)$ \\
\hline $\mathrm{J} 3423541+2519122$ & $19.43 \pm 0.29$ & $17.48 \pm 0.18$ & $1.92 \pm 0.34$ & $0.46 \pm 0.27$ & $1.07 \pm 0.32$ & $1.73 \pm 0.28$ & 19.2 & $\mathrm{M} 2$ (K0..M2) & $0.09(0.09 . .1 .45)$ \\
\hline $\mathrm{J} 3423681+2532175$ & $16.35 \pm 0.26$ & $15.04 \pm 0.17$ & $1.10 \pm 0.31$ & $1.50 \pm 0.17$ & $2.08 \pm 0.17$ & $2.28 \pm 0.17$ & 4.8 & M3 (M1..M4) & $0.82(0.21 . .1 .43)$ \\
\hline $\mathrm{J} 3423834+2528445$ & $18.63 \pm 0.27$ & $16.60 \pm 0.17$ & $1.79 \pm 0.32$ & $1.96 \pm 0.17$ & $2.44 \pm 0.18$ & $2.70 \pm 0.18$ & 2.7 & M5 (M5..M6) & $0.89(0.02 . .1 .21)$ \\
\hline $\mathrm{J} 3424093+2544004$ & $12.44 \pm 0.26$ & $11.75 \pm 0.17$ & $0.35 \pm 0.31$ & $0.98 \pm 0.17$ & $1.45 \pm 0.17$ & $1.50 \pm 0.17$ & 2.9 & $\mathrm{~K} 4$ (K3..K5) & $1.29(0.95 . .1 .48)$ \\
\hline $\mathrm{J} 3424262+2542554$ & $17.43 \pm 0.26$ & $15.84 \pm 0.17$ & $1.47 \pm 0.31$ & $1.65 \pm 0.17$ & $2.24 \pm 0.17$ & $2.42 \pm 0.17$ & 2.7 & M4 (M3..M4) & $0.46(0.14 . .1 .38)$ \\
\hline $\mathrm{J} 3424379+2532064$ & $16.09 \pm 0.26$ & $14.52 \pm 0.17$ & $1.41 \pm 0.31$ & $1.69 \pm 0.17$ & $2.24 \pm 0.17$ & $2.47 \pm 0.17$ & 3.7 & M4 (M3..M5) & $0.63(0.04 . .1 .29)$ \\
\hline $\mathrm{J} 3424641+2534273$ & $19.53 \pm 0.28$ & $17.61 \pm 0.17$ & $1.88 \pm 0.33$ & $1.30 \pm 0.20$ & $2.09 \pm 0.21$ & $2.22 \pm 0.24$ & 3.9 & M4 (M2..M4) & $0.13(0.13 . .1 .47)$ \\
\hline $\mathrm{J} 3424715+2530291$ & $18.51 \pm 0.27$ & $16.71 \pm 0.17$ & $1.62 \pm 0.32$ & $1.49 \pm 0.18$ & $2.18 \pm 0.18$ & $2.38 \pm 0.18$ & 1.2 & M4 (M3..M4) & $0.67(0.60 . .1 .41)$ \\
\hline $\mathrm{J} 3424775+2533352$ & $17.37 \pm 0.26$ & $15.61 \pm 0.17$ & $1.60 \pm 0.31$ & $1.99 \pm 0.17$ & $2.58 \pm 0.17$ & $2.81 \pm 0.17$ & 5.6 & M5 (M4..M5) & $0.59(0.16 . .1 .44)$ \\
\hline $\mathrm{J} 3424984+2542216$ & $19.88 \pm 0.31$ & $17.64 \pm 0.17$ & $2.24 \pm 0.35$ & $1.19 \pm 0.22$ & $1.70 \pm 0.25$ & $1.99 \pm 0.29$ & 12.4 & M4 (M0..M4) & $0.00(0.00 . .1 .45)$ \\
\hline $\mathrm{J} 3425056+2539126$ & $20.54 \pm 0.41$ & $17.95 \pm 0.17$ & $2.37 \pm 0.44$ & $0.90 \pm 0.25$ & $1.81 \pm 0.24$ & $2.26 \pm 0.24$ & 15.1 & M4 (M2..M6) & $0.83(0.05 . .1 .38)$ \\
\hline $\mathrm{J} 3425266+2542401$ & $18.70 \pm 0.27$ & $16.92 \pm 0.17$ & $1.73 \pm 0.32$ & $1.45 \pm 0.18$ & $2.09 \pm 0.19$ & $2.35 \pm 0.19$ & 1.4 & M4 (M3...M4) & $0.22(0.07 . .1 .12)$ \\
\hline $\mathrm{J} 3425524+2536143$ & $20.04 \pm 0.31$ & $17.71 \pm 0.17$ & $2.28 \pm 0.36$ & $1.62 \pm 0.19$ & $1.98 \pm 0.23$ & $2.69 \pm 0.21$ & 7.8 & M5 (M3..M6) & $0.19(0.00 . .1 .48)$ \\
\hline $\mathrm{J} 3425651+2549304$ & $19.11 \pm 0.28$ & $17.23 \pm 0.17$ & $1.83 \pm 0.33$ & $1.10 \pm 0.19$ & $1.64 \pm 0.21$ & $1.92 \pm 0.24$ & 8.9 & M3 (M0..M4) & $0.17(0.17 . .1 .14)$ \\
\hline $\mathrm{J} 3425701+2522459$ & $14.24 \pm 0.26$ & $13.16 \pm 0.17$ & $0.99 \pm 0.31$ & $1.10 \pm 0.17$ & $1.77 \pm 0.17$ & $1.87 \pm 0.17$ & 1.4 & M1 (K6..M2) & $0.36(0.12 . .1 .48)$ \\
\hline $\mathrm{J} 3430484+2528208$ & $13.08 \pm 0.26$ & $12.19 \pm 0.17$ & $0.74 \pm 0.31$ & $1.13 \pm 0.17$ & $1.77 \pm 0.17$ & $1.88 \pm 0.17$ & 2.5 & M0 (K4..M1) & $0.60(0.29 . .1 .49)$ \\
\hline $\mathrm{J} 3430500+2544353$ & $16.52 \pm 0.26$ & $14.96 \pm 0.17$ & $1.55 \pm 0.31$ & $1.56 \pm 0.17$ & $2.17 \pm 0.17$ & $2.43 \pm 0.17$ & 1.3 & M4 (M3..M4) & $0.02(0.02 . .1 .00)$ \\
\hline $\mathrm{J} 3430503+2552179$ & $11.75 \pm 0.26$ & $11.08 \pm 0.17$ & $0.38 \pm 0.31$ & $0.59 \pm 0.17$ & $0.86 \pm 0.17$ & $0.87 \pm 0.17$ & 1.8 & G7 (F9..K2) & $1.07(0.36 . .1 .49)$ \\
\hline $\mathrm{J} 3430622+2529517$ & $20.04 \pm 0.31$ & $17.75 \pm 0.17$ & $2.22 \pm 0.36$ & $1.67 \pm 0.19$ & $2.21 \pm 0.20$ & $2.56 \pm 0.22$ & 4.3 & M5 (M4..M5) & $0.23(0.23 . .1 .43)$ \\
\hline $\mathrm{J} 3430756+2534292$ & $16.58 \pm 0.26$ & $14.90 \pm 0.17$ & $1.47 \pm 0.31$ & $1.68 \pm 0.17$ & $2.20 \pm 0.17$ & $2.42 \pm 0.17$ & 2.7 & M4 (M3...M4) & $0.78(0.46 . .1 .41)$ \\
\hline $\mathrm{J} 3431003+2542085$ & $19.01 \pm 0.29$ & $16.96 \pm 0.17$ & $1.93 \pm 0.34$ & $1.79 \pm 0.18$ & $2.39 \pm 0.18$ & $2.69 \pm 0.18$ & 1.1 & M5 (M4..M5) & $0.45(0.21 . .1 .47)$ \\
\hline $\mathrm{J} 3431032+2530428$ & $19.84 \pm 0.31$ & $17.46 \pm 0.17$ & $2.28 \pm 0.36$ & $1.69 \pm 0.18$ & $2.22 \pm 0.19$ & $2.51 \pm 0.19$ & 6.0 & M5 (M4..M5) & $0.38(0.19 . .1 .13)$ \\
\hline $\mathrm{J} 3431184+2522105$ & $19.05 \pm 0.27$ & $17.03 \pm 0.17$ & $1.96 \pm 0.32$ & $1.03 \pm 0.19$ & $1.72 \pm 0.19$ & $1.75 \pm 0.21$ & 15.4 & M3 (K7..M3) & $0.22(0.21 . .1 .48)$ \\
\hline $\mathrm{J} 3431186+2536142$ & $18.81 \pm 0.27$ & $16.93 \pm 0.17$ & $1.88 \pm 0.32$ & $1.29 \pm 0.18$ & $1.85 \pm 0.19$ & $2.23 \pm 0.19$ & 6.2 & M4 (M1..M4) & $0.00(0.00 . .1 .42)$ \\
\hline $\mathrm{J} 3431446+2526543$ & $13.48 \pm 0.26$ & $12.65 \pm 0.17$ & $0.43 \pm 0.31$ & $0.71 \pm 0.17$ & $1.09 \pm 0.17$ & $1.08 \pm 0.17$ & 1.9 & $\mathrm{~K} 1(\mathrm{~K} 1 . . \mathrm{K} 6)$ & $1.49(0.25 . .1 .49)$ \\
\hline
\end{tabular}




\begin{tabular}{|c|c|c|c|c|c|c|c|c|c|}
\hline \multirow[b]{2}{*}{ 2MASS-ID } & \multicolumn{2}{|c|}{ measured magnitudes } & \multicolumn{4}{|c|}{ extinction corrected colors } & \multirow[b]{2}{*}{$\chi^{2}$} & \multirow[b]{2}{*}{$\mathrm{SpT}$} & \multirow[b]{2}{*}{$\mathrm{Av}$} \\
\hline & Rmag & Imag & R-I & I-J & $\mathrm{I}-\mathrm{H}$ & $\mathrm{I}-\mathrm{K}_{s}$ & & & \\
\hline $\mathrm{J} 3431502+2543208$ & $17.38 \pm 0.26$ & $15.85 \pm 0.17$ & $1.37 \pm 0.31$ & $1.00 \pm 0.17$ & $1.64 \pm 0.17$ & $1.89 \pm 0.18$ & 3.0 & M2 (M1..M3) & $0.60(0.04 . .1 .17)$ \\
\hline J3431652+2548596 & $20.20 \pm 0.36$ & $17.89 \pm 0.18$ & $2.30 \pm 0.41$ & $0.92 \pm 0.26$ & $1.81 \pm 0.27$ & $2.06 \pm 0.29$ & 13.3 & M4 (M0..M4) & $0.00(0.00 . .1 .24)$ \\
\hline J3431667+2532464 & $12.92 \pm 0.26$ & $12.12 \pm 0.17$ & $0.47 \pm 0.31$ & $0.82 \pm 0.17$ & $1.23 \pm 0.17$ & $1.32 \pm 0.17$ & 1.7 & K3 (K3..M0) & $1.22(0.01 . .1 .39)$ \\
\hline J3432032+2535585 & $9.78 \pm 0.26$ & $9.28 \pm 0.17$ & $0.26 \pm 0.31$ & $0.52 \pm 0.17$ & $0.66 \pm 0.17$ & $0.65 \pm 0.17$ & 2.3 & F7 (F7..K0) & $0.89(0.20 . .1 .21)$ \\
\hline $\mathrm{J} 3432179+2525183$ & $19.52 \pm 0.29$ & $17.59 \pm 0.17$ & $1.92 \pm 0.34$ & $1.10 \pm 0.21$ & $1.47 \pm 0.25$ & $1.89 \pm 0.25$ & 11.1 & M3 (M1..M4) & $0.01(0.01 . .1 .44)$ \\
\hline $\mathrm{J} 3432205+2524002$ & $17.46 \pm 0.26$ & $15.92 \pm 0.17$ & $1.37 \pm 0.31$ & $1.34 \pm 0.17$ & $1.90 \pm 0.18$ & $2.18 \pm 0.18$ & 1.1 & M3 (M2..M4) & $0.65(0.02 . .1 .43)$ \\
\hline $\mathrm{J} 3432258+2539059$ & $11.02 \pm 0.26$ & $10.48 \pm 0.17$ & $0.29 \pm 0.31$ & $0.50 \pm 0.17$ & $0.63 \pm 0.17$ & $0.65 \pm 0.17$ & 2.1 & F7 (F2..K0) & $0.95(0.18 . .1 .48)$ \\
\hline J3432526+2520459 & $12.17 \pm 0.26$ & $11.34 \pm 0.17$ & $0.46 \pm 0.31$ & $0.72 \pm 0.17$ & $0.96 \pm 0.17$ & $1.00 \pm 0.17$ & 3.0 & K0 (K0..K6) & $1.41(0.03 . .1 .41)$ \\
\hline $\mathrm{J} 3432712+2523152$ & $11.34 \pm 0.26$ & $10.58 \pm 0.17$ & $0.40 \pm 0.31$ & $0.56 \pm 0.17$ & $0.86 \pm 0.17$ & $0.87 \pm 0.17$ & 1.6 & G7 (G7..K6) & $1.35(0.01 . .1 .50)$ \\
\hline J3432774+2531447 & $12.65 \pm 0.26$ & $11.80 \pm 0.17$ & $0.53 \pm 0.31$ & $0.87 \pm 0.17$ & $1.37 \pm 0.17$ & $1.41 \pm 0.17$ & 1.5 & K4 (K3..M1) & $1.22(0.04 . .1 .46)$ \\
\hline $\mathrm{J} 3432981+2542351$ & $13.48 \pm 0.26$ & $12.65 \pm 0.17$ & $0.48 \pm 0.31$ & $0.78 \pm 0.17$ & $1.27 \pm 0.17$ & $1.31 \pm 0.17$ & 1.3 & K3 (K3..M0) & $1.33(0.12 . .1 .46)$ \\
\hline $\mathrm{J} 3433088+2531443$ & $21.18 \pm 0.61$ & $18.78 \pm 0.19$ & $2.40 \pm 0.64$ & $1.77 \pm 0.28$ & $2.63 \pm 0.27$ & $2.88 \pm 0.29$ & 1.8 & M6 (M4..M6) & $0.00(0.00 . .1 .33)$ \\
\hline J3433184+2522548 & $15.48 \pm 0.26$ & $14.50 \pm 0.17$ & $0.85 \pm 0.31$ & $1.14 \pm 0.17$ & $1.68 \pm 0.17$ & $1.81 \pm 0.17$ & 2.2 & M0 (K7...M2) & $0.51(0.08 . .1 .44)$ \\
\hline J3433401+2553374 & $19.29 \pm 0.26$ & $17.42 \pm 0.17$ & $1.71 \pm 0.31$ & $1.86 \pm 0.18$ & $2.57 \pm 0.18$ & $2.77 \pm 0.18$ & 3.0 & M5 (M4..M5) & $0.58(0.47 . .1 .37)$ \\
\hline J3433448+2536145 & $17.71 \pm 0.26$ & $15.83 \pm 0.17$ & $1.85 \pm 0.31$ & $1.82 \pm 0.17$ & $2.44 \pm 0.17$ & $2.72 \pm 0.17$ & 1.2 & M5 (M4..M5) & $0.11(0.01 . .1 .26)$ \\
\hline J3433674+2522135 & $19.87 \pm 0.32$ & $17.89 \pm 0.18$ & $1.74 \pm 0.37$ & $1.79 \pm 0.19$ & $2.58 \pm 0.20$ & $2.74 \pm 0.20$ & 2.4 & M5 (M5..M5) & $0.91(0.54 . .1 .29)$ \\
\hline $\mathrm{J} 3412705+2523594$ & $19.94 \pm 0.33$ & $17.42 \pm 0.17$ & $2.49 \pm 0.37$ & $1.16 \pm 0.19$ & $1.77 \pm 0.21$ & $1.91 \pm 0.23$ & 23.0 & M4 (M0..M4) & $0.11(0.11 . .1 .41)$ \\
\hline $\mathrm{J} 3412939+2522530$ & $20.84 \pm 0.56$ & $17.93 \pm 0.19$ & $2.91 \pm 0.59$ & $1.76 \pm 0.20$ & $2.58 \pm 0.21$ & $2.65 \pm 0.22$ & 7.3 & M6 (M4..M6) & $0.00(0.00 . .1 .43)$ \\
\hline $\mathrm{J} 3414220+2552506$ & $20.09 \pm 0.28$ & $17.49 \pm 0.17$ & $2.48 \pm 0.33$ & $1.04 \pm 0.21$ & $1.79 \pm 0.21$ & $1.70 \pm 0.28$ & 26.1 & M4 (M1..M5) & $0.46(0.06 . .1 .50)$ \\
\hline $\mathrm{J} 3422643+2532396$ & $20.45 \pm 0.38$ & $17.75 \pm 0.17$ & $2.70 \pm 0.42$ & $1.73 \pm 0.19$ & $2.32 \pm 0.20$ & $2.59 \pm 0.23$ & 9.2 & M6 (M4..M6) & $0.00(0.00 . .1 .46)$ \\
\hline $\mathrm{J} 3423524+2553112$ & $20.86 \pm 0.46$ & $18.00 \pm 0.18$ & $2.66 \pm 0.50$ & $1.08 \pm 0.23$ & $1.68 \pm 0.26$ & $0.68 \pm \ldots$ & 14.5 & M4 (M2..M6) & $0.77(0.21 . .1 .06)$ \\
\hline $\mathrm{J} 3423808+2519220$ & $14.85 \pm 0.26$ & $13.51 \pm 0.17$ & $1.34 \pm 0.31$ & $1.29 \pm 0.17$ & $1.92 \pm 0.17$ & $2.18 \pm 0.17$ & 1.1 & M3 (M1..M3) & $0.00(0.00 . .0 .95)$ \\
\hline $\mathrm{J} 3430535+2546411$ & $15.93 \pm 0.26$ & $14.30 \pm 0.17$ & $1.63 \pm 0.31$ & $1.58 \pm 0.17$ & $2.09 \pm 0.17$ & $2.36 \pm 0.17$ & 1.3 & M4 (M3..M4) & $0.00(0.00 . .0 .69)$ \\
\hline J3431777+2553361 & $12.09 \pm 0.26$ & $11.16 \pm 0.17$ & $0.67 \pm 0.31$ & $1.22 \pm 0.17$ & $1.78 \pm 0.17$ & $1.89 \pm 0.17$ & 4.3 & M0 (M0..M2) & $0.95(0.12 . .1 .15)$ \\
\hline $\mathrm{J} 3433413+2535261$ & $16.15 \pm 0.26$ & $14.44 \pm 0.17$ & $1.64 \pm 0.31$ & $1.55 \pm 0.17$ & $2.14 \pm 0.17$ & $2.35 \pm 0.17$ & 1.2 & M4 (M3..M4) & $0.25(0.05 . .1 .11)$ \\
\hline $\mathrm{J} 3433521+2524312$ & $16.09 \pm 0.26$ & $14.36 \pm 0.17$ & $1.67 \pm 0.31$ & $1.53 \pm 0.17$ & $2.14 \pm 0.17$ & $2.33 \pm 0.17$ & 1.3 & M4 (M3..M4) & $0.22(0.01 . .1 .09)$ \\
\hline $\mathrm{J} 3433666+2547007$ & $16.11 \pm 0.26$ & $14.41 \pm 0.17$ & $1.68 \pm 0.31$ & $1.52 \pm 0.17$ & $2.11 \pm 0.17$ & $2.34 \pm 0.17$ & 1.2 & M4 (M3..M4) & $0.06(0.06 . .0 .92)$ \\
\hline
\end{tabular}


Tabelle A2 Extinction corrected photometry and spectral type for objects not consistent with the Pleiades sample, but listed in the 2MASS catalog. These objects are most likely background objects - stars or distant galaxies. As described in the text the grid of possible extinctions for the $\chi^{2}$ minimization is predefined $A_{V}=[0 \ldots 2]$. According to the limiting magnitude of the 2 mass catalog $\left(K_{s} \approx 15\right)$ and of our survey most objects with 2 mass counterpart (listed in this table) are red objects. Bluer objects without 2 mass counterpart detected in this study are shown in Tbl. A3 Some of the object are located near a saturated star (or are a saturated star itself), near the edge of the image, or near illumination effects visible in Fig. 1 and Fig. 2 Typically this is indicated by a large value of $\chi^{2}$ and leads often to a wrong spectral type and unreliable extinctions. For the Pleiades membership candidates (Tbl. A1 these objects are removed from the list but this is not taken into account for the rest of the sources. Furthermore a wide range of possible spectral types indicates that the minimum found in the $\chi^{2}$ matrix is not unique, indicating that two different sets of SpT/Av values (late type star at low extinction, or early type star at high extinction) have nearly the same probability. As mentioned already in the text the spectral classification of such objects (often also indicated by a large $\chi^{2}$ value) is doubtful.

\begin{tabular}{|c|c|c|c|c|c|c|c|c|c|}
\hline \multirow[b]{2}{*}{ 2MASS-ID } & \multicolumn{2}{|c|}{ measured magnitudes } & \multicolumn{4}{|c|}{ extinction corrected colors } & \multirow[b]{2}{*}{$\chi^{2}$} & \multirow[b]{2}{*}{ SpT } & \multirow[b]{2}{*}{ Av } \\
\hline & Rmag & Imag & R-I & I-J & $\mathrm{I}-\mathrm{H}$ & $\mathrm{I}-\mathrm{K}_{s}$ & & & \\
\hline J3405901+2545498 & $18.08 \pm 0.27$ & $18.53 \pm 0.19$ & $-0.82 \pm 0.33$ & $1.48 \pm 0.24$ & $1.92 \pm 0.27$ & $2.61 \pm 0.24$ & 61.4 & K4 (G6..M2) & $1.39(0.14 . .1 .42)$ \\
\hline $\mathrm{J} 3405925+2526146$ & $18.88 \pm 0.28$ & $19.51 \pm 0.30$ & $-1.14 \pm 0.41$ & $2.79 \pm 0.32$ & $3.14 \pm 0.34$ & $3.20 \pm 0.35$ & 98.3 & M0 (G3..M5) & $1.90(0.18 . .1 .92)$ \\
\hline $\mathrm{J} 3405932+2523454$ & $17.85 \pm 0.27$ & $17.59 \pm 0.18$ & $0.15 \pm 0.32$ & $0.76 \pm 0.23$ & $1.61 \pm 0.23$ & $1.85 \pm 0.24$ & 7.4 & K4 (G7..K6) & $0.40(0.18 . .1 .43)$ \\
\hline $\mathrm{J} 3405951+2524049$ & $19.19 \pm 0.32$ & $18.79 \pm 0.24$ & $0.38 \pm 0.40$ & $5.65 \pm 0.24$ & $6.16 \pm 0.24$ & $6.24 \pm 0.24$ & 135.8 & L5 (M8..L5) & $0.04(0.04 . .1 .41)$ \\
\hline $\mathrm{J} 3405971+2534375$ & $18.91 \pm 0.29$ & $20.69 \pm 0.85$ & $-1.89 \pm 0.90$ & $8.76 \pm 0.85$ & $9.07 \pm 0.85$ & $9.12 \pm 0.85$ & 106.4 & L5 (M7.5..L5) & $0.42(0.07 . .1 .25)$ \\
\hline $\mathrm{J} 3410003+2546035$ & $17.81 \pm 0.27$ & $17.67 \pm 0.17$ & $0.15 \pm 0.32$ & $0.80 \pm 0.24$ & $1.08 \pm 0.33$ & $1.58 \pm \ldots$ & 2.7 & K0 (F6..K2) & $0.00(0.00 . .0 .72)$ \\
\hline $\mathrm{J} 3410029+2548560$ & $16.27 \pm 0.26$ & $14.80 \pm 0.17$ & $1.33 \pm 0.31$ & $-1.87 \pm 0.21$ & $-1.61 \pm 0.28$ & $-1.07 \pm 0.23$ & 102.5 & B0 (B0..F0) & $0.52(0.21 . .0 .93)$ \\
\hline $\mathrm{J} 3410058+2535137$ & $18.35 \pm 0.27$ & $18.04 \pm 0.18$ & $0.03 \pm 0.33$ & $1.27 \pm 0.21$ & $1.67 \pm$ & $1.65 \pm 0.30$ & 8.9 & K4 (G9..K7) & $1.05(0.02 . .1 .48)$ \\
\hline $\mathrm{J} 3410117+2543046$ & $18.05 \pm 0.27$ & $17.16 \pm 0.17$ & $0.89 \pm 0.32$ & $3.43 \pm 0.17$ & $4.22 \pm 0.17$ & $4.39 \pm 0.17$ & 81.9 & M8 (M6.5..L0.5) & $0.00(0.00 . .1 .44)$ \\
\hline $\mathrm{J} 3410143+2528062$ & $17.57 \pm 0.26$ & $20.09 \pm 0.53$ & $-2.92 \pm 0.59$ & $4.91 \pm 0.53$ & $5.02 \pm 0.53$ & $5.11 \pm 0.53$ & 183.7 & M0 (F7..M6) & $1.51(0.10 . .1 .58)$ \\
\hline $\mathrm{J} 3410146+2551236$ & $16.43 \pm 0.26$ & $16.53 \pm 0.17$ & $-0.60 \pm 0.31$ & $1.98 \pm 0.17$ & $2.41 \pm 0.17$ & $2.46 \pm 0.17$ & 109.7 & M0 (K4..M5) & $1.87(0.06 . .1 .87)$ \\
\hline J3410182+2519536 & $19.79 \pm 0.30$ & $19.53 \pm 0.18$ & $0.02 \pm 0.35$ & $2.79 \pm 0.21$ & $3.17 \pm 0.25$ & $3.86 \pm 0.22$ & 111.9 & M5 (M0..M9.5) & $0.88(0.07 . .1 .47)$ \\
\hline $\mathrm{J} 3410209+2546403$ & $19.10 \pm 0.29$ & $18.47 \pm 0.18$ & $0.40 \pm 0.34$ & $1.38 \pm 0.24$ & $1.64 \pm 0.32$ & $2.34 \pm 0.28$ & 10.7 & M0 (K4..M2) & $0.89(0.43 . .1 .24)$ \\
\hline $\mathrm{J} 3410220+2537298$ & $16.51 \pm 0.26$ & $16.00 \pm 0.17$ & $0.37 \pm 0.31$ & $0.54 \pm 0.18$ & $0.84 \pm 0.18$ & $0.93 \pm 0.19$ & 1.2 & G7 (F7..K1) & $0.53(0.08 . .1 .26)$ \\
\hline $\mathrm{J} 3410230+2535330$ & $17.89 \pm 0.27$ & $17.65 \pm 0.18$ & $0.20 \pm 0.32$ & $1.06 \pm 0.21$ & $1.51 \pm 0.25$ & $1.64 \pm 0.28$ & 4.6 & K4 (K0..K6) & $0.14(0.01 . .0 .88)$ \\
\hline $\mathrm{J} 3410231+2530001$ & $17.80 \pm 0.27$ & $17.07 \pm 0.17$ & $0.36 \pm 0.32$ & $0.86 \pm 0.18$ & $1.48 \pm 0.19$ & $1.54 \pm 0.20$ & 1.9 & K4 (K4..M0) & $1.43(0.21 . .1 .43)$ \\
\hline $\mathrm{J} 3410247+2541090$ & $15.38 \pm 0.26$ & $14.81 \pm 0.17$ & $0.36 \pm 0.31$ & $0.71 \pm 0.17$ & $1.00 \pm 0.17$ & $1.09 \pm 0.17$ & 1.9 & $\mathrm{~K} 0$ (G3..K2) & $0.77(0.41 . .1 .49)$ \\
\hline $\mathrm{J} 3410251+2549044$ & $15.27 \pm 0.26$ & $14.69 \pm 0.17$ & $0.36 \pm 0.31$ & $0.81 \pm 0.17$ & $1.00 \pm 0.17$ & $1.03 \pm 0.17$ & 3.8 & K0 (G0..K4) & $0.85(0.06 . .1 .50)$ \\
\hline $\mathrm{J} 3410273+2546591$ & $18.33 \pm 0.27$ & $17.55 \pm 0.17$ & $0.39 \pm 0.32$ & $0.56 \pm 0.22$ & $1.63 \pm 0.20$ & $1.55 \pm 0.23$ & 5.5 & K4 (K3..M0) & $1.45(0.48 . .1 .46)$ \\
\hline $\mathrm{J} 3410298+2542511$ & $17.50 \pm 0.26$ & $16.81 \pm 0.17$ & $0.48 \pm 0.32$ & $0.87 \pm 0.18$ & $1.27 \pm 0.19$ & $1.22 \pm 0.21$ & 2.6 & K3 (G7..K6) & $0.77(0.03 . .1 .48)$ \\
\hline J3410304+2551016 & $19.38 \pm 0.30$ & $17.73 \pm 0.18$ & $1.65 \pm 0.35$ & $2.09 \pm 0.18$ & $2.43 \pm 0.20$ & $2.73 \pm 0.20$ & 5.5 & M5 (M4..M5) & $0.00(0.00 . .1 .40)$ \\
\hline $\mathrm{J} 3410351+2533458$ & $16.81 \pm 0.26$ & $16.23 \pm 0.17$ & $0.10 \pm 0.31$ & $-0.88 \pm 0.22$ & $-0.23 \pm 0.22$ & $-0.16 \pm 0.25$ & 15.3 & B6 (B4..F6) & $1.80(0.04 . .1 .80)$ \\
\hline $\mathrm{J} 3410352+2545536$ & $17.33 \pm 0.26$ & $16.86 \pm 0.17$ & $0.43 \pm 0.31$ & $0.93 \pm 0.18$ & $1.17 \pm 0.21$ & $1.34 \pm 0.22$ & 3.3 & K3 (F9..K4) & $0.14(0.14 . .1 .46)$ \\
\hline $\mathrm{J} 3410368+2548260$ & $14.50 \pm 0.26$ & $13.87 \pm 0.17$ & $0.40 \pm 0.31$ & $0.70 \pm 0.17$ & $1.00 \pm 0.17$ & $1.04 \pm 0.17$ & 2.0 & K0 (G2..K5) & $0.88(0.06 . .1 .47)$ \\
\hline $\mathrm{J} 3410377+2541368$ & $17.44 \pm 0.26$ & $16.61 \pm 0.17$ & $0.48 \pm 0.31$ & $0.82 \pm 0.18$ & $1.20 \pm 0.19$ & $1.33 \pm 0.19$ & 1.7 & K3 (K3..M0) & $1.29(0.01 . .1 .29)$ \\
\hline $\mathrm{J} 3410395+2544020$ & $17.24 \pm 0.26$ & $16.70 \pm 0.17$ & $0.43 \pm 0.31$ & $0.92 \pm 0.18$ & $1.31 \pm 0.19$ & $1.56 \pm 0.19$ & 2.1 & K4 (K0..K5) & $0.41(0.17 . .1 .12)$ \\
\hline $\mathrm{J} 3410417+2534382$ & $15.50 \pm 0.26$ & $14.85 \pm 0.17$ & $0.52 \pm 0.31$ & $0.79 \pm 0.17$ & $1.26 \pm 0.17$ & $1.26 \pm 0.17$ & 1.6 & K3 (G9..K6) & $0.53(0.06 . .1 .33)$ \\
\hline $\mathrm{J} 3410421+2525077$ & $18.32 \pm 0.27$ & $16.80 \pm 0.17$ & $1.21 \pm 0.32$ & $0.42 \pm 0.19$ & $1.05 \pm 0.20$ & $1.51 \pm 0.20$ & 12.0 & $\mathrm{~K} 7$ (K4..M2) & $1.19(0.24 . .1 .37)$ \\
\hline $\mathrm{J} 3410423+2533408$ & $16.88 \pm 0.26$ & $16.30 \pm 0.17$ & $0.38 \pm 0.31$ & $0.74 \pm 0.18$ & $0.89 \pm 0.19$ & $1.13 \pm 0.19$ & 3.0 & $\mathrm{~K} 0(\mathrm{G} 3 . . \mathrm{K} 4)$ & $0.73(0.07 . .1 .46)$ \\
\hline $\mathrm{J} 3410425+2546334$ & $19.53 \pm 0.34$ & $18.39 \pm 0.19$ & $0.81 \pm 0.39$ & $1.09 \pm 0.27$ & $1.78 \pm 0.28$ & $1.79 \pm 0.32$ & 1.4 & M0 (M0..M3) & $1.24(0.06 . .1 .41)$ \\
\hline J3410444+2520165 & $17.26 \pm 0.26$ & $16.53 \pm 0.17$ & $0.32 \pm 0.31$ & $0.48 \pm 0.18$ & $0.34 \pm 0.21$ & $0.49 \pm 0.22$ & 3.8 & F5 (F5..G6) & $1.54(0.57 . .1 .54)$ \\
\hline $\mathrm{J} 3410468+2539566$ & $18.12 \pm 0.27$ & $17.79 \pm 0.17$ & $0.33 \pm 0.32$ & $0.94 \pm 0.24$ & $1.36 \pm 0.29$ & $1.96 \pm \ldots$ & 1.9 & $\mathrm{~K} 4(\mathrm{~K} 0 . . \mathrm{K} 4)$ & $0.00(0.00 . .1 .06)$ \\
\hline $\mathrm{J} 3410484+2551572$ & $17.41 \pm 0.26$ & $16.57 \pm 0.17$ & $0.84 \pm 0.31$ & $1.23 \pm 0.18$ & $1.66 \pm 0.18$ & $1.74 \pm 0.19$ & 4.2 & M0 (K2..M1) & $0.00(0.00 . .1 .28)$ \\
\hline $\mathrm{J} 3410491+2548208$ & $12.58 \pm 0.26$ & $12.01 \pm 0.17$ & $0.40 \pm 0.31$ & $0.74 \pm 0.17$ & $0.98 \pm 0.17$ & $1.03 \pm 0.17$ & 2.7 & $\mathrm{~K} 0$ (G0..K2) & $0.63(0.32 . .1 .36)$ \\
\hline $\mathrm{J} 3410493+2528124$ & $17.41 \pm 0.26$ & $16.59 \pm 0.17$ & $0.43 \pm 0.31$ & $0.51 \pm 0.18$ & $0.83 \pm 0.19$ & $0.88 \pm 0.20$ & 1.3 & G7 (G7..K5) & $1.49(0.16 . .1 .49)$ \\
\hline J3410504+2553334 & $17.01 \pm 0.26$ & $16.16 \pm 0.17$ & $0.49 \pm 0.31$ & $0.88 \pm 0.17$ & $1.22 \pm 0.18$ & $1.26 \pm 0.18$ & 2.7 & K3 (K1..K7) & $1.35(0.42 . .1 .48)$ \\
\hline $\mathrm{J} 3410506+2550394$ & $20.37 \pm 0.43$ & $19.25 \pm 0.25$ & $0.88 \pm 0.49$ & $2.38 \pm 0.28$ & $3.01 \pm 0.30$ & $3.10 \pm 0.32$ & 19.0 & M5 (M3..M7.5) & $0.86(0.06 . .1 .39)$ \\
\hline $\mathrm{J} 3410510+2529116$ & $18.41 \pm 0.27$ & $17.40 \pm 0.17$ & $0.54 \pm 0.32$ & $-0.02 \pm 0.25$ & $0.60 \pm 0.27$ & $0.71 \pm 0.29$ & 4.2 & G0 (F8..K5) & $1.78(0.24 . .1 .78)$ \\
\hline $\mathrm{J} 3410512+2534482$ & $16.81 \pm 0.26$ & $15.98 \pm 0.17$ & $0.41 \pm 0.31$ & $0.67 \pm 0.17$ & $1.07 \pm 0.18$ & & 1.3 & K1 (K1..K6) & $1.54(0.39 . .1 .54)$ \\
\hline $\mathrm{J} 3410523+2530094$ & $17.65 \pm 0.27$ & $16.97 \pm 0.17$ & $0.47 \pm 0.32$ & $0.91 \pm 0.18$ & $1.44 \pm 0.19$ & $1.38 \pm 0.21$ & 2.0 & K4 (K3..K6) & $0.78(0.44 . .1 .15)$ \\
\hline $\mathrm{J} 3410529+2520072$ & $21.14 \pm 0.67$ & $17.94 \pm 0.18$ & $3.07 \pm 0.69$ & $1.47 \pm 0.21$ & $1.65 \pm 0.26$ & $1.99 \pm 0.26$ & 19.5 & M4 (M1..M5) & $0.49(0.17 . .1 .26)$ \\
\hline $\mathrm{J} 3410548+2553069$ & $16.46 \pm 0.26$ & $15.58 \pm 0.17$ & $0.47 \pm 0.31$ & $0.71 \pm 0.17$ & $1.06 \pm 0.17$ & $1.05 \pm 0.18$ & 2.2 & $\mathrm{~K} 1(\mathrm{~K} 1 . . \mathrm{K} 7)$ & $1.54(0.29 . .1 .56)$ \\
\hline $\mathrm{J} 3410549+2530411$ & $15.29 \pm 0.26$ & $14.67 \pm 0.17$ & $0.26 \pm 0.31$ & $0.48 \pm 0.17$ & $0.66 \pm 0.17$ & $0.67 \pm 0.17$ & 1.7 & F7 (F7..K0) & $1.34(0.38 . .1 .37)$ \\
\hline $\mathrm{J} 3410560+2536198$ & $18.05 \pm 0.27$ & $17.08 \pm 0.17$ & $0.85 \pm 0.32$ & $1.05 \pm 0.19$ & $1.74 \pm 0.19$ & $1.82 \pm 0.20$ & 1.3 & M0 (K5..M2) & $0.48(0.05 . .1 .50)$ \\
\hline $\mathrm{J} 3410582+2548388$ & $17.19 \pm 0.26$ & $16.39 \pm 0.17$ & $0.41 \pm 0.31$ & $0.49 \pm 0.18$ & $0.81 \pm 0.19$ & $0.94 \pm 0.19$ & 1.2 & G7 (G4..K6) & $1.51(0.08 . .1 .60)$ \\
\hline J3410604+2521159 & $20.30 \pm 0.38$ & $18.48 \pm 0.20$ & $1.44 \pm 0.43$ & $0.88 \pm 0.30$ & $1.40 \pm 0.34$ & $1.99 \pm 0.29$ & 3.7 & M2 (M2..M4) & $1.43(0.16 . .1 .43)$ \\
\hline $\mathrm{J} 3410630+2526382$ & $19.27 \pm 0.29$ & $17.50 \pm 0.18$ & $1.64 \pm 0.34$ & $1.21 \pm 0.19$ & $1.88 \pm 0.20$ & $1.93 \pm 0.21$ & 4.1 & M3 (M1..M4) & $0.51(0.08 . .1 .40)$ \\
\hline $\mathrm{J} 3410656+2548204$ & $17.31 \pm 0.26$ & $16.75 \pm 0.17$ & $0.39 \pm 0.31$ & $0.71 \pm 0.18$ & $0.98 \pm 0.21$ & $1.04 \pm 0.23$ & 2.0 & $\mathrm{~K} 0(\mathrm{~F} 7 . . \mathrm{K} 2)$ & $0.61(0.28 . .1 .48)$ \\
\hline $\mathrm{J} 3410662+2533153$ & $17.82 \pm 0.26$ & $16.46 \pm 0.17$ & $1.34 \pm 0.31$ & $1.30 \pm 0.18$ & $2.03 \pm 0.17$ & $2.14 \pm 0.18$ & 1.5 & M3 (M1..M3) & $0.09(0.09 . .1 .24)$ \\
\hline $\mathrm{J} 3410666+2530326$ & $18.51 \pm 0.27$ & $17.71 \pm 0.17$ & $0.65 \pm 0.32$ & $0.73 \pm 0.23$ & $1.52 \pm 0.24$ & $1.32 \pm 0.31$ & 2.1 & K5 (K0..K7) & $0.60(0.23 . .1 .28)$ \\
\hline $\mathrm{J} 3410688+2528126$ & $16.94 \pm 0.26$ & $16.10 \pm 0.17$ & $0.52 \pm 0.31$ & $0.73 \pm 0.18$ & $1.25 \pm 0.18$ & $1.29 \pm 0.18$ & 1.1 & $\mathrm{~K} 3$ (K2..K7) & $1.16(0.47 . .1 .28)$ \\
\hline $\mathrm{J} 3410690+2551347$ & $17.42 \pm 0.26$ & $16.46 \pm 0.17$ & $0.57 \pm 0.31$ & $0.71 \pm 0.18$ & $1.13 \pm 0.18$ & $1.00 \pm 0.19$ & 3.4 & $\mathrm{~K} 2(\mathrm{~K} 0 . . \mathrm{K} 7)$ & $1.48(0.26 . .1 .48)$ \\
\hline $\mathrm{J} 3410712+2524136$ & $17.47 \pm 0.26$ & $16.08 \pm 0.17$ & $1.18 \pm 0.31$ & $-0.86 \pm 0.22$ & $-0.48 \pm 0.27$ & $0.08 \pm 0.25$ & 44.9 & F0 (B3..G9) & $0.76(0.22 . .1 .47)$ \\
\hline $\mathrm{J} 3410726+2544316$ & $15.82 \pm 0.26$ & $15.24 \pm 0.17$ & $0.34 \pm 0.31$ & $0.61 \pm 0.17$ & $0.87 \pm 0.17$ & $0.90 \pm 0.18$ & 1.8 & G7 (F6..K0) & $0.92(0.38 . .1 .49)$ \\
\hline $\mathrm{J} 3410728+2522327$ & $16.53 \pm 0.26$ & $16.17 \pm 0.17$ & $0.36 \pm 0.31$ & $0.94 \pm 0.17$ & $1.47 \pm 0.18$ & $1.39 \pm 0.19$ & 3.0 & $\mathrm{~K} 4(\mathrm{~K} 1 . . \mathrm{K} 4)$ & $0.00(0.00 . .0 .62)$ \\
\hline J3410735+2525404 & $15.41 \pm 0.26$ & $14.64 \pm 0.17$ & $0.50 \pm 0.31$ & $0.79 \pm 0.17$ & $1.25 \pm 0.17$ & $1.29 \pm 0.17$ & 1.5 & K3 (K0..K7) & $0.99(0.42 . .1 .50)$ \\
\hline $\mathrm{J} 3410736+2528361$ & $18.94 \pm 0.27$ & $17.60 \pm 0.17$ & $1.22 \pm 0.32$ & $0.99 \pm 0.21$ & $1.70 \pm 0.22$ & $2.02 \pm 0.22$ & 1.6 & M2 (M0..M2) & $0.46(0.24 . .1 .06)$ \\
\hline $\mathrm{J} 3410741+2543113$ & $14.44 \pm 0.26$ & $13.88 \pm 0.17$ & $0.25 \pm 0.31$ & $0.47 \pm 0.17$ & $0.64 \pm 0.17$ & $0.71 \pm 0.17$ & 1.6 & F7 (F7..K1) & $1.17(0.20 . .1 .43)$ \\
\hline $\mathrm{J} 3410743+2552017$ & $17.61 \pm 0.26$ & $16.55 \pm 0.17$ & $1.06 \pm 0.31$ & $1.09 \pm 0.18$ & $1.73 \pm 0.18$ & $1.66 \pm 0.19$ & 3.6 & M1 (K3..M1) & $0.00(0.00 . .1 .41)$ \\
\hline $\mathrm{J} 3410746+2543031$ & $18.99 \pm 0.28$ & $17.89 \pm 0.17$ & $0.59 \pm 0.33$ & $1.33 \pm 0.19$ & $1.65 \pm 0.22$ & $2.04 \pm 0.20$ & 7.2 & M0 (M0..M4) & $1.91(0.11 . .1 .91)$ \\
\hline $\mathrm{J} 3410750+2533288$ & $14.90 \pm 0.26$ & $14.19 \pm 0.17$ & $0.47 \pm 0.31$ & $0.91 \pm 0.17$ & $1.37 \pm 0.17$ & $1.45 \pm 0.17$ & 1.7 & $\mathrm{~K} 4$ (K1..K5) & $0.89(0.67 . .1 .47)$ \\
\hline $\mathrm{J} 3410762+2526319$ & $15.95 \pm 0.26$ & $15.27 \pm 0.17$ & $0.30 \pm 0.31$ & $0.49 \pm 0.17$ & $0.61 \pm 0.18$ & $0.67 \pm 0.18$ & 1.9 & F7 (F7..K1) & $1.43(0.26 . .1 .50)$ \\
\hline $\mathrm{J} 3410767+2527388$ & $12.27 \pm 0.26$ & $11.83 \pm 0.17$ & $0.04 \pm 0.31$ & $0.20 \pm 0.17$ & $0.19 \pm 0.17$ & $0.17 \pm 0.17$ & 1.2 & A4 (A3..G2) & $1.51(0.06 . .1 .61)$ \\
\hline $\mathrm{J} 3410769+2552295$ & $18.16 \pm 0.26$ & $17.28 \pm 0.17$ & $0.71 \pm 0.32$ & $1.31 \pm 0.18$ & $1.79 \pm 0.20$ & $1.76 \pm 0.22$ & 5.9 & M0 (K4...M2) & $0.65(0.06 . .1 .34)$ \\
\hline J3410811+2524119 & $16.05 \pm 0.26$ & $15.32 \pm 0.17$ & $0.44 \pm 0.31$ & $0.33 \pm 0.17$ & $0.83 \pm 0.18$ & $0.82 \pm 0.18$ & 1.5 & G6 (F8..K0) & $1.08(0.61 . .1 .47)$ \\
\hline $\mathrm{J} 3410816+2539035$ & $16.75 \pm 0.26$ & $15.56 \pm 0.17$ & $0.84 \pm 0.31$ & $1.10 \pm 0.17$ & $1.70 \pm 0.17$ & $1.83 \pm 0.17$ & 1.6 & M0 (M0..M2) & $1.34(0.61 . .1 .42)$ \\
\hline $\mathrm{J} 3410822+2545596$ & $17.14 \pm 0.26$ & $15.99 \pm 0.17$ & $0.88 \pm 0.31$ & $1.07 \pm 0.17$ & $1.71 \pm 0.17$ & $1.78 \pm 0.18$ & 1.6 & M0 (M0..M2) & $1.05(0.29 . .1 .17)$ \\
\hline $\mathrm{J} 3410828+2527093$ & $17.44 \pm 0.26$ & $16.05 \pm 0.17$ & $1.30 \pm 0.31$ & $1.00 \pm 0.17$ & $1.70 \pm 0.18$ & $1.92 \pm 0.18$ & 2.0 & M2 (K7...M2) & $0.34(0.16 . .1 .44)$ \\
\hline $\mathrm{J} 3410832+2530145$ & $15.71 \pm 0.26$ & $15.14 \pm 0.17$ & $0.41 \pm 0.31$ & $0.63 \pm 0.17$ & $1.01 \pm 0.18$ & $1.07 \pm 0.18$ & 1.2 & $\mathrm{~K} 0$ (F7..K3) & $0.58(0.10 . .1 .47)$ \\
\hline $\mathrm{J} 3410834+2548246$ & $18.53 \pm 0.27$ & $17.72 \pm 0.18$ & $0.52 \pm 0.32$ & $0.70 \pm 0.23$ & $1.44 \pm 0.23$ & $1.29 \pm \ldots$ & 1.4 & K4 (K1..M0) & $1.11(0.19 . .1 .48)$ \\
\hline $\mathrm{J} 3410840+2543133$ & $15.98 \pm 0.26$ & $16.14 \pm 0.17$ & $-0.16 \pm 0.31$ & $1.03 \pm 0.17$ & $1.48 \pm 0.18$ & $1.56 \pm 0.18$ & 18.5 & K3 (G1..K4) & $0.00(0.00 . .1 .37)$ \\
\hline $\mathrm{J} 3410867+2529467$ & $17.78 \pm 0.26$ & $16.43 \pm 0.17$ & $1.18 \pm 0.31$ & $1.41 \pm 0.18$ & $2.08 \pm 0.17$ & $2.23 \pm 0.18$ & 2.9 & M3 (M1..M4) & $0.67(0.04 . .1 .29)$ \\
\hline $\mathrm{J} 3410869+2546171$ & $18.09 \pm 0.27$ & $17.34 \pm 0.17$ & $0.44 \pm 0.32$ & $1.31 \pm 0.18$ & $1.90 \pm 0.18$ & $2.05 \pm 0.19$ & 10.4 & M0 (K7..M3) & $1.18(0.10 . .1 .49)$ \\
\hline
\end{tabular}




\begin{tabular}{|c|c|c|c|c|c|c|c|c|c|}
\hline \multirow[b]{2}{*}{ 2MASS-ID } & \multicolumn{2}{|c|}{ measured magnitudes } & & extinction c & ected colors & & & & \\
\hline & Rmag & Imag & R-I & I-J & $\mathrm{I}-\mathrm{H}$ & $\mathrm{I}-\mathrm{K}_{s}$ & $\chi^{2}$ & SpT & $\mathrm{Av}$ \\
\hline $\mathrm{J} 3410887+2526323$ & $18.13 \pm 0.27$ & $16.96 \pm 0.17$ & $0.85 \pm 0.32$ & $1.06 \pm 0.18$ & $1.70 \pm 0.18$ & $1.84 \pm 0.19$ & 1.2 & M0 (M0..M2) & $1.20(0.51 . .1 .33)$ \\
\hline $\mathrm{J} 3410910+2547435$ & $19.91 \pm 0.33$ & $19.90 \pm 0.36$ & $0.01 \pm 0.48$ & $3.06 \pm 0.39$ & $2.62 \pm$ & $3.73 \pm \ldots$ & 28.0 & M4 (K1..M5) & $0.00(0.00 . .1 .40)$ \\
\hline $\mathrm{J} 3410915+2525181$ & $16.70 \pm 0.26$ & $16.13 \pm 0.17$ & $0.41 \pm 0.31$ & $0.56 \pm 0.18$ & $0.99 \pm 0.18$ & $0.68 \pm 0.21$ & 4.1 & G7 (F6..K2) & $0.58(0.33 . .1 .36)$ \\
\hline $\mathrm{J} 3410919+2522355$ & $18.44 \pm 0.28$ & $17.20 \pm 0.17$ & $0.95 \pm 0.32$ & $1.59 \pm 0.18$ & $2.23 \pm 0.18$ & $2.27 \pm 0.18$ & 9.7 & M3 (M1..M4) & $1.07(0.38 . .1 .50)$ \\
\hline $\mathrm{J} 3410945+2543157$ & $18.20 \pm 0.27$ & $17.51 \pm 0.17$ & $0.21 \pm 0.32$ & $1.08 \pm 0.19$ & $1.43 \pm 0.21$ & $1.64 \pm 0.20$ & 5.5 & $\mathrm{~K} 4$ (K4..M1) & $1.78(0.30 . .1 .78)$ \\
\hline $\mathrm{J} 3410948+2545007$ & $16.72 \pm 0.26$ & $16.14 \pm 0.17$ & $0.23 \pm 0.31$ & $0.37 \pm 0.18$ & $0.53 \pm 0.19$ & $0.55 \pm 0.20$ & 1.3 & F5 (F5..G7) & $1.32(0.28 . .1 .50)$ \\
\hline $\mathrm{J} 3410966+2519261$ & $18.69 \pm 0.27$ & $18.06 \pm 0.18$ & $0.43 \pm 0.33$ & $1.36 \pm 0.21$ & $1.93 \pm 0.24$ & $2.09 \pm 0.26$ & 8.9 & M0 (K4..M2) & $0.78(0.01 . .1 .47)$ \\
\hline $\mathrm{J} 3411031+2549137$ & $15.38 \pm 0.26$ & $14.73 \pm 0.17$ & $0.51 \pm 0.31$ & $0.86 \pm 0.17$ & $1.23 \pm 0.17$ & $1.24 \pm 0.17$ & 2.6 & K3 (G7..K6) & $0.54(0.17 . .1 .37)$ \\
\hline $\mathrm{J} 3411041+2543430$ & $18.65 \pm 0.27$ & $18.23 \pm 0.18$ & $0.04 \pm 0.33$ & $1.18 \pm 0.23$ & $1.65 \pm 0.26$ & $1.94 \pm \ldots$ & 7.2 & K4 (K3..M2) & $1.44(0.05 . .1 .44)$ \\
\hline $\mathrm{J} 3411067+2551418$ & $15.54 \pm 0.26$ & $14.87 \pm 0.17$ & $0.49 \pm 0.31$ & $0.85 \pm 0.17$ & $1.23 \pm 0.17$ & $1.28 \pm 0.17$ & 2.1 & K3 (G9..K7) & $0.67(0.01 . .1 .44)$ \\
\hline $\mathrm{J} 3411074+2541336$ & $18.04 \pm 0.26$ & $17.42 \pm 0.17$ & $0.39 \pm 0.32$ & $0.61 \pm 0.21$ & $1.04 \pm 0.25$ & $1.25 \pm 0.25$ & 1.2 & K1 (G7..K6) & $0.85(0.04 . .1 .39)$ \\
\hline $\mathrm{J} 3411099+2544225$ & $16.90 \pm 0.26$ & $16.34 \pm 0.17$ & $0.50 \pm 0.31$ & $0.86 \pm 0.17$ & $1.47 \pm 0.18$ & $1.34 \pm 0.19$ & 2.3 & K4 (G7..K6) & $0.21(0.06 . .1 .35)$ \\
\hline $\mathrm{J} 3411103+2552186$ & $19.72 \pm 0.31$ & $18.98 \pm 0.23$ & $0.28 \pm 0.39$ & $1.36 \pm 0.31$ & $1.98 \pm 0.33$ & $2.36 \pm 0.31$ & 9.6 & M0 (K4..M4) & $1.72(0.06 . .1 .89)$ \\
\hline $\mathrm{J} 3411139+2542571$ & $18.90 \pm 0.27$ & $17.57 \pm 0.17$ & $0.90 \pm 0.32$ & $0.65 \pm 0.20$ & $1.46 \pm 0.20$ & $1.40 \pm 0.24$ & 2.3 & K7 (K5..M2) & $1.63(0.19 . .1 .63)$ \\
\hline $\mathrm{J} 3411168+2526237$ & $18.32 \pm 0.27$ & $18.80 \pm 0.23$ & $-0.70 \pm 0.35$ & $1.79 \pm 0.27$ & $2.24 \pm 0.32$ & $2.13 \pm \ldots$ & 34.6 & K4 (G1..M1) & $0.81(0.07 . .1 .17)$ \\
\hline $\mathrm{J} 3411169+2550317$ & $19.54 \pm 0.30$ & $18.34 \pm 0.18$ & $0.63 \pm 0.35$ & $1.16 \pm 0.23$ & $1.78 \pm 0.24$ & $2.01 \pm 0.24$ & 3.1 & M0 (M0..M2) & $2.13(1.66 . .2 .13)$ \\
\hline $\mathrm{J} 3411179+2542187$ & $17.09 \pm 0.26$ & $16.16 \pm 0.17$ & $0.80 \pm 0.31$ & $0.59 \pm 0.19$ & $1.27 \pm 0.19$ & $1.75 \pm 0.19$ & 4.9 & K7 (K0..K7) & $0.46(0.36 . .1 .38)$ \\
\hline $\mathrm{J} 3411192+2546266$ & $19.97 \pm 0.33$ & $18.95 \pm 0.21$ & $0.85 \pm 0.39$ & $5.38 \pm 0.21$ & $5.75 \pm 0.21$ & $5.82 \pm 0.21$ & 99.8 & L5 (M9.5..L5) & $0.63(0.22 . .1 .44)$ \\
\hline $\mathrm{J} 3411218+2542500$ & $20.14 \pm 0.36$ & $18.18 \pm 0.18$ & $1.81 \pm 0.40$ & $1.79 \pm 0.20$ & $2.55 \pm 0.21$ & $2.68 \pm 0.21$ & 1.8 & M5 (M4..M5) & $0.58(0.22 . .1 .49)$ \\
\hline $\mathrm{J} 3411224+2541354$ & $17.76 \pm 0.26$ & $16.96 \pm 0.17$ & $0.72 \pm 0.31$ & $0.65 \pm 0.20$ & $1.37 \pm 0.21$ & $1.74 \pm 0.21$ & 2.9 & K7 (K0..K7) & $0.28(0.28 . .1 .44)$ \\
\hline $\mathrm{J} 3411227+2535267$ & $16.81 \pm 0.26$ & $16.37 \pm 0.17$ & $0.42 \pm 0.31$ & $0.62 \pm 0.18$ & $1.01 \pm 0.19$ & $1.05 \pm 0.21$ & 1.3 & $\mathrm{~K} 0(\mathrm{~F} 6 . . \mathrm{K} 0)$ & $0.04(0.04 . .1 .00)$ \\
\hline $\mathrm{J} 3411242+2547168$ & $17.60 \pm 0.26$ & $16.37 \pm 0.17$ & $0.81 \pm 0.31$ & $1.26 \pm 0.17$ & $1.87 \pm 0.17$ & $1.90 \pm 0.18$ & 4.6 & M1 (M0..M4) & $1.57(0.00 . .1 .57)$ \\
\hline $\mathrm{J} 3411256+2522211$ & $21.61 \pm 1.03$ & $17.73 \pm 0.18$ & $3.65 \pm 1.04$ & $1.26 \pm 0.21$ & $2.10 \pm 0.20$ & $2.16 \pm 0.21$ & 17.5 & M4 (M0..M6) & $0.83(0.37 . .1 .48)$ \\
\hline $\mathrm{J} 3411261+2545241$ & $19.73 \pm 0.31$ & $18.41 \pm 0.19$ & $0.77 \pm 0.36$ & $1.00 \pm 0.25$ & $1.69 \pm 0.26$ & $2.00 \pm 0.25$ & 1.7 & M0 (M0..M2) & $2.08(1.56 . .2 .08)$ \\
\hline $\mathrm{J} 3411288+2533030$ & $19.71 \pm 0.30$ & $19.48 \pm 0.25$ & $-0.06 \pm 0.39$ & $2.36 \pm 0.29$ & $2.99 \pm 0.31$ & $2.43 \pm \ldots$ & 42.3 & M3 (K6..M6) & $1.08(0.14 . .1 .08)$ \\
\hline $\mathrm{J} 3411304+2551061$ & $18.72 \pm 0.27$ & $17.42 \pm 0.17$ & $1.04 \pm 0.32$ & $1.44 \pm 0.18$ & $2.12 \pm 0.19$ & $2.36 \pm 0.19$ & 5.5 & M3 (M2..M4) & $0.98(0.13 . .1 .38)$ \\
\hline $\mathrm{J} 3411343+2536316$ & $16.79 \pm 0.26$ & $16.09 \pm 0.17$ & $0.40 \pm 0.31$ & $0.91 \pm 0.18$ & $1.39 \pm 0.18$ & $1.53 \pm 0.18$ & 1.7 & K4 (K3..M0) & $1.16(0.08 . .1 .37)$ \\
\hline $\mathrm{J} 3411344+2523093$ & $18.33 \pm 0.27$ & $15.90 \pm 0.17$ & $2.43 \pm 0.32$ & $1.94 \pm 0.17$ & $2.57 \pm 0.17$ & $2.90 \pm 0.17$ & 1.9 & M6 (M6..M6) & $0.00(0.00 . .0 .27)$ \\
\hline $\mathrm{J} 3411363+2542377$ & $19.34 \pm 0.29$ & $18.05 \pm 0.18$ & $1.02 \pm 0.34$ & $1.54 \pm 0.20$ & $2.10 \pm 0.21$ & $2.36 \pm 0.22$ & 5.6 & M3 (M1..M4) & $1.04(0.56 . .1 .48)$ \\
\hline $\mathrm{J} 3411376+2525044$ & $15.08 \pm 0.26$ & $14.48 \pm 0.17$ & $0.22 \pm 0.31$ & $0.41 \pm 0.17$ & $0.54 \pm 0.17$ & $0.54 \pm 0.17$ & 1.7 & F5 (F5..G9) & $1.42(0.43 . .1 .42)$ \\
\hline $\mathrm{J} 3411412+2523344$ & $18.81 \pm 0.28$ & $17.47 \pm 0.17$ & $1.04 \pm 0.33$ & $0.51 \pm 0.21$ & $0.98 \pm 0.25$ & $1.68 \pm 0.22$ & 8.7 & K7 (K3..M2) & $1.12(0.18 . .1 .50)$ \\
\hline $\mathrm{J} 3411414+2551372$ & $18.14 \pm 0.27$ & $17.34 \pm 0.17$ & $0.37 \pm 0.32$ & $0.94 \pm 0.19$ & $1.36 \pm 0.20$ & $1.58 \pm 0.21$ & 2.3 & K4 (K3..M0) & $1.61(0.28 . .1 .70)$ \\
\hline $\mathrm{J} 3411432+2548449$ & $16.04 \pm 0.26$ & $15.38 \pm 0.17$ & $0.50 \pm 0.31$ & $0.82 \pm 0.17$ & $1.23 \pm 0.17$ & $1.28 \pm 0.18$ & 1.8 & K3 (G8..K6) & $0.60(0.09 . .1 .43)$ \\
\hline $\mathrm{J} 3411443+2536203$ & $17.16 \pm 0.26$ & $16.30 \pm 0.17$ & $0.63 \pm 0.31$ & $1.23 \pm 0.17$ & $1.77 \pm 0.18$ & $1.94 \pm 0.18$ & 4.9 & M0 (K6..M2) & $0.85(0.32 . .1 .38)$ \\
\hline $\mathrm{J} 3411444+2540249$ & $17.75 \pm 0.26$ & $17.05 \pm 0.17$ & $0.46 \pm 0.31$ & $0.01 \pm 0.22$ & $0.95 \pm 0.22$ & $0.89 \pm 0.25$ & 6.5 & G6 (F5..K3) & $0.92(0.27 . .1 .46)$ \\
\hline $\mathrm{J} 3411471+2540385$ & $17.68 \pm 0.26$ & $17.04 \pm 0.17$ & $0.24 \pm 0.31$ & $0.32 \pm 0.20$ & $0.39 \pm 0.25$ & $0.29 \pm 0.31$ & 1.8 & F3 (A8..G7) & $1.52(0.22 . .1 .57)$ \\
\hline $\mathrm{J} 3411477+2540138$ & $17.81 \pm 0.26$ & $17.05 \pm 0.17$ & $0.34 \pm 0.31$ & $-0.13 \pm 0.23$ & $0.37 \pm 0.26$ & $-0.42 \pm \ldots$ & 3.2 & F1 (A8..K1) & $1.59(0.07 . .1 .59)$ \\
\hline $\mathrm{J} 3411481+2534047$ & $16.75 \pm 0.26$ & $16.18 \pm 0.17$ & $0.40 \pm 0.31$ & $0.66 \pm 0.17$ & $0.92 \pm 0.19$ & $1.14 \pm 0.19$ & 1.8 & $\mathrm{~K} 0$ (F8..K2) & $0.64(0.34 . .1 .49)$ \\
\hline $\mathrm{J} 3411519+2528337$ & $17.14 \pm 0.26$ & $16.45 \pm 0.17$ & $0.34 \pm 0.31$ & $0.35 \pm 0.18$ & $0.59 \pm 0.20$ & $0.72 \pm 0.20$ & 1.1 & F8 (F5..G8) & $1.34(0.66 . .1 .48)$ \\
\hline $\mathrm{J} 3411525+2537565$ & $18.56 \pm 0.27$ & $17.99 \pm 0.18$ & $0.27 \pm 0.32$ & $0.91 \pm 0.23$ & $1.45 \pm \ldots$ & $1.68 \pm 0.27$ & 2.4 & K4 (K3..M0) & $1.13(0.06 . .1 .46)$ \\
\hline $\mathrm{J} 3411528+2548036$ & $16.12 \pm 0.26$ & $15.49 \pm 0.17$ & $0.51 \pm 0.31$ & $0.84 \pm 0.17$ & $1.23 \pm 0.18$ & $1.27 \pm 0.18$ & 2.1 & K3 (G8..K4) & $0.48(0.12 . .1 .37)$ \\
\hline $\mathrm{J} 3411529+2552179$ & $17.99 \pm 0.26$ & $16.95 \pm 0.17$ & $0.76 \pm 0.31$ & $1.15 \pm 0.18$ & $1.74 \pm 0.18$ & $1.86 \pm 0.19$ & 2.4 & M0 (M0..M2) & $1.05(0.30 . .1 .29)$ \\
\hline $\mathrm{J} 3411546+2535321$ & $19.35 \pm 0.28$ & $18.34 \pm 0.18$ & $0.73 \pm 0.34$ & $1.68 \pm 0.21$ & $2.37 \pm 0.22$ & $2.46 \pm 0.23$ & 15.4 & M3 (M1..M4) & $1.03(0.15 . .1 .47)$ \\
\hline $\mathrm{J} 3411555+2547204$ & $18.63 \pm 0.27$ & $17.25 \pm 0.17$ & $0.98 \pm 0.32$ & $1.12 \pm 0.18$ & $1.71 \pm 0.18$ & $1.91 \pm 0.19$ & 1.3 & M1 (M1..M3) & $1.53(0.40 . .1 .53)$ \\
\hline $\mathrm{J} 3411569+2536523$ & $18.30 \pm 0.27$ & $17.58 \pm 0.17$ & $0.34 \pm 0.32$ & $0.52 \pm 0.22$ & $0.61 \pm 0.30$ & $1.56 \pm \ldots$ & 1.7 & G4 (F8..K4) & $1.46(0.14 . .1 .46)$ \\
\hline $\mathrm{J} 3411577+2540087$ & $17.30 \pm 0.26$ & $16.47 \pm 0.17$ & $0.44 \pm 0.31$ & $0.06 \pm 0.21$ & $0.47 \pm 0.22$ & $0.68 \pm 0.22$ & 3.4 & F6 (F6..K1) & $1.51(0.34 . .1 .51)$ \\
\hline $\mathrm{J} 3411578+2524171$ & $18.25 \pm 0.27$ & $18.45 \pm 0.20$ & $-0.43 \pm 0.34$ & $1.40 \pm 0.25$ & $1.92 \pm 0.29$ & $2.19 \pm 0.29$ & 26.8 & K4 (G7...M1) & $0.87(0.03 . .1 .40)$ \\
\hline $\mathrm{J} 3411594+2521472$ & $17.69 \pm 0.26$ & $16.72 \pm 0.17$ & $0.68 \pm 0.32$ & $1.25 \pm 0.18$ & $1.82 \pm 0.18$ & $1.83 \pm 0.18$ & 5.0 & M0 (M0..M2) & $1.07(0.41 . .1 .47)$ \\
\hline $\mathrm{J} 3411609+2528256$ & $17.61 \pm 0.26$ & $17.05 \pm 0.17$ & $0.40 \pm 0.31$ & $0.69 \pm 0.19$ & $1.06 \pm 0.21$ & $0.95 \pm 0.27$ & 2.1 & K0 (G3..K2) & $0.58(0.12 . .1 .39)$ \\
\hline $\mathrm{J} 3411624+2540352$ & $18.24 \pm 0.27$ & $16.76 \pm 0.17$ & $1.32 \pm 0.32$ & $1.37 \pm 0.17$ & $2.00 \pm 0.18$ & $2.15 \pm 0.18$ & 1.4 & M3 (M1..M3) & $0.60(0.39 . .1 .48)$ \\
\hline $\mathrm{J} 3411626+2524011$ & $16.61 \pm 0.26$ & $16.03 \pm 0.17$ & $0.49 \pm 0.31$ & $0.83 \pm 0.17$ & $1.25 \pm 0.18$ & $1.28 \pm 0.18$ & 1.8 & K3 (G6..K3) & $0.32(0.14 . .1 .33)$ \\
\hline $\mathrm{J} 3411663+2544542$ & $15.76 \pm 0.26$ & $15.25 \pm 0.17$ & $0.41 \pm 0.31$ & $0.71 \pm 0.17$ & $1.00 \pm 0.17$ & $1.03 \pm 0.18$ & 2.1 & $\mathrm{~K} 0(\mathrm{~F} 5 . . \mathrm{K} 2)$ & $0.40(0.22 . .1 .46)$ \\
\hline $\mathrm{J} 3411712+2550120$ & $15.97 \pm 0.26$ & $15.33 \pm 0.17$ & $0.28 \pm 0.31$ & $0.46 \pm 0.17$ & $0.63 \pm 0.18$ & $0.69 \pm 0.18$ & 1.4 & F7 (F7..K2) & $1.33(0.10 . .1 .39)$ \\
\hline $\mathrm{J} 3411713+2539440$ & $17.76 \pm 0.26$ & $17.14 \pm 0.17$ & $0.35 \pm 0.31$ & $0.71 \pm 0.20$ & $1.01 \pm 0.21$ & $1.08 \pm 0.24$ & 1.6 & K0 (G4..K4) & $1.00(0.09 . .1 .47)$ \\
\hline $\mathrm{J} 3411729+2542401$ & $15.45 \pm 0.26$ & $14.52 \pm 0.17$ & $0.68 \pm 0.31$ & $-0.73 \pm 0.20$ & $-0.00 \pm 0.21$ & $0.40 \pm 0.20$ & 30.1 & F0 (A1..G3) & $0.93(0.01 . .1 .32)$ \\
\hline $\mathrm{J} 3411737+2546434$ & $16.15 \pm 0.26$ & $15.50 \pm 0.17$ & $0.42 \pm 0.31$ & $0.67 \pm 0.17$ & $1.06 \pm 0.17$ & $0.98 \pm 0.18$ & 2.3 & $\mathrm{~K} 0(\mathrm{G} 4 . . \mathrm{K} 5)$ & $0.84(0.03 . .1 .41)$ \\
\hline $\mathrm{J} 3411746+2549546$ & $18.89 \pm 0.27$ & $17.43 \pm 0.17$ & $1.29 \pm 0.32$ & $0.94 \pm 0.20$ & $1.65 \pm 0.20$ & $2.01 \pm 0.20$ & 2.6 & M2 (M0..M2) & $0.63(0.25 . .1 .39)$ \\
\hline $\mathrm{J} 3411770+2525582$ & $17.04 \pm 0.26$ & $16.71 \pm 0.17$ & $0.23 \pm 0.31$ & $0.83 \pm 0.18$ & $1.08 \pm 0.20$ & $1.10 \pm 0.22$ & 3.8 & $\mathrm{~K} 0$ (G2..K2) & $0.38(0.14 . .1 .32)$ \\
\hline $\mathrm{J} 3411772+2538581$ & $18.58 \pm 0.27$ & $17.36 \pm 0.17$ & $0.92 \pm 0.32$ & $0.27 \pm 0.24$ & $1.20 \pm 0.22$ & $1.77 \pm 0.20$ & 10.8 & $\mathrm{~K} 7$ (K4..M2) & $1.16(0.01 . .1 .16)$ \\
\hline $\mathrm{J} 3411779+2542178$ & $17.55 \pm 0.26$ & $16.73 \pm 0.17$ & $0.42 \pm 0.31$ & $0.33 \pm 0.18$ & $0.59 \pm 0.20$ & $0.67 \pm 0.22$ & 1.2 & G0 (F7..G7) & $1.48(1.04 . .1 .50)$ \\
\hline $\mathrm{J} 3411793+2542413$ & $15.95 \pm 0.26$ & $14.93 \pm 0.17$ & $0.53 \pm 0.31$ & $-0.37 \pm 0.18$ & $0.21 \pm 0.18$ & $0.23 \pm 0.18$ & 12.1 & F0 (A9..G5) & $1.84(0.22 . .1 .84)$ \\
\hline $\mathrm{J} 3411803+2536431$ & $18.95 \pm 0.27$ & $17.58 \pm 0.17$ & $1.36 \pm 0.32$ & $0.87 \pm 0.21$ & $1.94 \pm 0.21$ & $1.31 \pm 0.34$ & 10.0 & M2 (K2..M2) & $0.00(0.00 . .1 .48)$ \\
\hline $\mathrm{J} 3411814+2540077$ & $15.85 \pm 0.26$ & $15.25 \pm 0.17$ & $0.53 \pm 0.31$ & $0.92 \pm 0.17$ & $1.36 \pm 0.18$ & $1.38 \pm 0.17$ & 2.2 & K4 (G9..K6) & $0.29(0.06 . .1 .38)$ \\
\hline $\mathrm{J} 3411833+2550428$ & $13.53 \pm 0.26$ & $12.92 \pm 0.17$ & $0.34 \pm 0.31$ & $0.64 \pm 0.17$ & $0.86 \pm 0.17$ & $0.88 \pm 0.17$ & 2.4 & G7 (F6..K0) & $0.99(0.60 . .1 .49)$ \\
\hline $\mathrm{J} 3411843+2521212$ & $18.65 \pm 0.27$ & $19.07 \pm 0.22$ & $-0.78 \pm 0.35$ & $2.07 \pm 0.25$ & $2.87 \pm 0.26$ & $2.87 \pm 0.28$ & 86.2 & M0 (K0..M5) & $1.40(0.21 . .1 .47)$ \\
\hline $\mathrm{J} 3411868+2547005$ & $14.41 \pm 0.26$ & $13.82 \pm 0.17$ & $0.41 \pm 0.31$ & $0.69 \pm 0.17$ & $1.00 \pm 0.17$ & $1.05 \pm 0.17$ & 1.8 & $\mathrm{~K} 0$ (G1..K2) & $0.71(0.34 . .1 .38)$ \\
\hline $\mathrm{J} 3411873+2547412$ & $16.56 \pm 0.26$ & $15.98 \pm 0.17$ & $0.36 \pm 0.31$ & $0.70 \pm 0.18$ & $0.99 \pm 0.18$ & $1.11 \pm 0.18$ & 1.7 & $\mathrm{~K} 0(\mathrm{G} 3 . . \mathrm{K} 3)$ & $0.85(0.32 . .1 .50)$ \\
\hline $\mathrm{J} 3411881+2526504$ & $16.15 \pm 0.26$ & $15.56 \pm 0.17$ & $0.25 \pm 0.31$ & $0.32 \pm 0.17$ & $0.54 \pm 0.18$ & $0.54 \pm 0.18$ & 1.1 & F5 (F5..G7) & $1.28(0.31 . .1 .28)$ \\
\hline J3411885+2540291 & $17.88 \pm 0.26$ & $16.80 \pm 0.17$ & $0.78 \pm 0.31$ & $1.13 \pm 0.18$ & $1.81 \pm 0.18$ & $1.79 \pm 0.18$ & 2.8 & M0 (M0..M2) & $1.14(0.51 . .1 .36)$ \\
\hline $\mathrm{J} 3411891+2538048$ & $16.81 \pm 0.26$ & $16.16 \pm 0.17$ & $0.47 \pm 0.31$ & $0.92 \pm 0.17$ & $1.36 \pm 0.18$ & $1.46 \pm 0.18$ & 1.7 & $\mathrm{~K} 4(\mathrm{~K} 0 . . \mathrm{K} 4)$ & $0.68(0.56 . .1 .48)$ \\
\hline $\mathrm{J} 3411921+2531185$ & $18.38 \pm 0.27$ & $17.11 \pm 0.17$ & $1.17 \pm 0.32$ & $0.48 \pm 0.20$ & $1.18 \pm 0.22$ & $1.37 \pm 0.24$ & 7.8 & $\mathrm{~K} 7(\mathrm{~K} 0 . . \mathrm{K} 7)$ & $0.34(0.07 . .1 .23)$ \\
\hline $\mathrm{J} 3411937+2535034$ & $19.02 \pm 0.27$ & $18.38 \pm 0.18$ & $0.27 \pm 0.33$ & $1.15 \pm 0.23$ & $1.50 \pm$ & $1.40 \pm 0.37$ & 4.4 & K4 (K3..M2) & $1.38(0.09 . .1 .38)$ \\
\hline J3411944+2543568 & $18.69 \pm 0.27$ & $18.02 \pm 0.18$ & $0.19 \pm 0.32$ & $0.93 \pm 0.21$ & $1.64 \pm 0.23$ & $2.09 \pm$. & 4.0 & K4 (K3..M1) & $1.80(0.04 . .1 .80)$ \\
\hline $\mathrm{J} 3412013+2525316$ & $17.77 \pm 0.26$ & $16.74 \pm 0.17$ & $0.56 \pm 0.31$ & $0.53 \pm 0.18$ & $0.80 \pm 0.20$ & $0.69 \pm 0.22$ & 3.6 & G7 (G4..K3) & $1.78(0.61 . .1 .78)$ \\
\hline $\mathrm{J} 3412023+2549261$ & $18.24 \pm 0.27$ & $17.69 \pm 0.18$ & $0.43 \pm 0.32$ & $0.85 \pm 0.22$ & $1.42 \pm 0.26$ & $1.52 \pm 0.29$ & 1.2 & $\mathrm{~K} 4$ (K1..K5) & $0.46(0.30 . .1 .23)$ \\
\hline $\mathrm{J} 3412026+2547562$ & $18.48 \pm 0.27$ & $17.48 \pm 0.17$ & $0.72 \pm 0.32$ & $1.14 \pm 0.19$ & $1.73 \pm 0.20$ & $1.93 \pm 0.21$ & 2.5 & M0 (M0..M2) & $1.05(0.43 . .1 .24)$ \\
\hline $\mathrm{J} 3412065+2530275$ & $16.61 \pm 0.26$ & $15.78 \pm 0.17$ & $0.53 \pm 0.31$ & $0.92 \pm 0.17$ & $1.35 \pm 0.17$ & $1.40 \pm 0.17$ & 2.1 & $\mathrm{~K} 4(\mathrm{~K} 2 . . \mathrm{K} 7)$ & $1.12(0.62 . .1 .48)$ \\
\hline $\mathrm{J} 3412081+2541156$ & $15.98 \pm 0.26$ & $15.25 \pm 0.17$ & $0.36 \pm 0.31$ & $0.94 \pm 0.17$ & $1.43 \pm 0.17$ & $1.52 \pm 0.17$ & 2.3 & K4 (K4..K5) & $1.38(1.09 . .1 .48)$ \\
\hline $\mathrm{J} 3412093+2532190$ & $20.17 \pm 0.34$ & $18.20 \pm 0.18$ & $1.89 \pm 0.38$ & $1.39 \pm 0.23$ & $1.74 \pm 0.28$ & $2.44 \pm 0.26$ & 4.5 & M4 (M2..M4) & $0.28(0.08 . .1 .47)$ \\
\hline $\mathrm{J} 3412097+2527418$ & $18.62 \pm 0.27$ & $17.57 \pm 0.18$ & $0.86 \pm 0.32$ & $0.81 \pm 0.21$ & $1.15 \pm 0.26$ & $1.59 \pm 0.25$ & 3.0 & K7 (K0..K7) & $0.70(0.49 . .1 .48)$ \\
\hline $\mathrm{J} 3412101+2538345$ & $14.08 \pm 0.26$ & $13.65 \pm 0.17$ & $0.43 \pm 0.31$ & $-1.65 \pm 0.18$ & $-1.32 \pm 0.19$ & $-1.03 \pm 0.19$ & 77.8 & B0 (B0..B7) & $0.00(0.00 . .0 .69)$ \\
\hline $\mathrm{J} 3412110+2541503$ & $17.93 \pm 0.26$ & $17.76 \pm 0.17$ & $-0.02 \pm 0.32$ & $1.28 \pm 0.19$ & $1.55 \pm 0.24$ & $1.77 \pm 0.25$ & 13.7 & K4 (G7..K6) & $0.72(0.33 . .1 .49)$ \\
\hline
\end{tabular}


T. Eisenbeiss et al.: New brown dwarf candidates in the Pleiades

\begin{tabular}{|c|c|c|c|c|c|c|c|c|c|}
\hline \multirow[b]{2}{*}{ 2MASS-ID } & \multicolumn{2}{|c|}{ measured magnitudes } & & extinction c & ected colors & & & & \\
\hline & Rmag & Imag & R-I & I-J & $\mathrm{I}-\mathrm{H}$ & $\mathrm{I}-\mathrm{K}_{s}$ & $\chi^{2}$ & SpT & $\mathrm{Av}$ \\
\hline $\mathrm{J} 3412135+2535061$ & $18.82 \pm 0.27$ & $17.28 \pm 0.17$ & $1.32 \pm 0.32$ & $1.31 \pm 0.18$ & $1.94 \pm 0.19$ & $2.23 \pm 0.19$ & 1.1 & M3 (M2..M4) & $0.83(0.01 . .1 .39)$ \\
\hline J3412136+2554066 & $19.10 \pm 0.26$ & $14.02 \pm 0.17$ & $4.78 \pm 0.31$ & $0.99 \pm 0.17$ & $1.48 \pm 0.17$ & $1.54 \pm 0.17$ & 286.2 & M6 (M4..M8) & $1.14(0.03 . .1 .44)$ \\
\hline $\mathrm{J} 3412164+2544161$ & $17.02 \pm 0.26$ & $16.44 \pm 0.17$ & $0.36 \pm 0.31$ & $0.63 \pm 0.18$ & $1.02 \pm 0.19$ & $1.13 \pm 0.19$ & 1.1 & K0 (G9..K4) & $0.82(0.15 . .1 .20)$ \\
\hline $\mathrm{J} 3412180+2528289$ & $17.05 \pm 0.26$ & $17.04 \pm 0.17$ & $-0.00 \pm 0.31$ & $0.23 \pm 0.22$ & $-0.70 \pm$. & $1.23 \pm$ & 1.2 & A5 (B8..A7) & $0.03(0.03 . .1 .29)$ \\
\hline $\mathrm{J} 3412181+2553253$ & $17.62 \pm 0.26$ & $16.53 \pm 0.17$ & $1.01 \pm 0.31$ & $1.03 \pm 0.18$ & $1.64 \pm 0.19$ & $1.99 \pm 0.19$ & 1.5 & M1 (K5..M2) & $0.29(0.04 . .1 .47)$ \\
\hline $\mathrm{J} 3412189+2526321$ & $21.87 \pm 1.32$ & $18.40 \pm 0.20$ & $3.10 \pm 1.33$ & $1.37 \pm 0.23$ & $2.05 \pm$ & $2.25 \pm$ & 3.6 & M4 (M2..M6) & $1.39(0.13 . .1 .39)$ \\
\hline J3412194+2548408 & $17.83 \pm 0.26$ & $17.14 \pm 0.17$ & $0.46 \pm 0.31$ & $0.71 \pm 0.19$ & $1.50 \pm 0.20$ & $1.49 \pm 0.22$ & 1.9 & K4 (K1..K5) & $0.87(0.51 . .1 .47)$ \\
\hline $\mathrm{J} 3412226+2540298$ & $12.83 \pm 0.26$ & $12.22 \pm 0.17$ & $0.46 \pm 0.31$ & $0.93 \pm 0.17$ & $1.38 \pm 0.17$ & $1.46 \pm 0.17$ & 1.8 & K4 (K2..K5) & $0.59(0.29 . .1 .23)$ \\
\hline $\mathrm{J} 3412230+2537083$ & $17.17 \pm 0.26$ & $16.56 \pm 0.17$ & $0.42 \pm 0.31$ & $0.69 \pm 0.18$ & $1.07 \pm 0.19$ & $0.94 \pm 0.21$ & 2.7 & K0 (F9..K4) & $0.72(0.17 . .1 .42)$ \\
\hline J3412234+2533109 & $18.73 \pm 0.27$ & $17.77 \pm 0.17$ & $0.94 \pm 0.32$ & $1.02 \pm 0.21$ & $1.77 \pm 0.24$ & $1.58 \pm 0.31$ & 2.5 & M0 (K4..M0) & $0.08(0.08 . .1 .37)$ \\
\hline J3412237+2528197 & $17.13 \pm 0.26$ & $16.69 \pm 0.17$ & $0.39 \pm 0.31$ & $0.74 \pm 0.19$ & $1.06 \pm 0.21$ & $0.93 \pm 0.25$ & 2.9 & K0 (G0..K2) & $0.19(0.06 . .1 .29)$ \\
\hline $\mathrm{J} 3412253+2530490$ & $18.60 \pm 0.27$ & $17.67 \pm 0.17$ & $0.75 \pm 0.32$ & $1.28 \pm 0.19$ & $1.79 \pm 0.21$ & $1.71 \pm 0.24$ & 5.1 & M0 (K4..M2) & $0.64(0.19 . .1 .39)$ \\
\hline J3412254+2540114 & $17.28 \pm 0.26$ & $16.40 \pm 0.17$ & $0.87 \pm 0.31$ & $0.70 \pm 0.18$ & $1.34 \pm 0.18$ & $1.55 \pm 0.20$ & 1.6 & K7 (G8..K7) & $0.07(0.07 . .1 .46)$ \\
\hline $\mathrm{J} 3412287+2542535$ & $17.38 \pm 0.26$ & $16.90 \pm 0.17$ & $0.35 \pm 0.31$ & $0.55 \pm 0.20$ & $0.95 \pm 0.23$ & $0.81 \pm 0.28$ & 1.7 & G7 (F7..K2) & $0.47(0.02 . .1 .26)$ \\
\hline $\mathrm{J} 3412289+2525315$ & $17.49 \pm 0.26$ & $16.65 \pm 0.17$ & $0.38 \pm 0.31$ & $0.98 \pm 0.18$ & $1.52 \pm 0.18$ & $1.39 \pm 0.18$ & 3.7 & K4 (K4..M1) & $1.69(0.46 . .1 .69)$ \\
\hline J3412297+2548365 & $17.60 \pm 0.26$ & $16.90 \pm 0.17$ & $0.54 \pm 0.31$ & $0.82 \pm$ & & 1.42 & 1.2 & & \\
\hline J3412308+2520455 & $18.28 \pm 0.27$ & $17.90 \pm 0.18$ & $0.03 \pm 0.32$ & $0.90 \pm 0.23$ & $1.34 \pm 0.26$ & $2.08 \pm 0.22$ & 14.6 & K4 (K1..M1) & $1.31(0.07 . .1 .47)$ \\
\hline $\mathrm{J} 3412309+2524265$ & $13.09 \pm 0.26$ & $12.50 \pm 0.17$ & $0.30 \pm 0.31$ & $0.46 \pm 0.17$ & $0.66 \pm 0.17$ & $0.64 \pm 0.17$ & 1.7 & & $1.08(0.49 . .1 .35)$ \\
\hline $\mathrm{J} 3412315+2519032$ & $17.12 \pm 0.26$ & $16.09 \pm 0.17$ & $0.51 \pm 0.31$ & $0.23 \pm 0.18$ & $0.57 \pm 0.18$ & $0.65 \pm 0.19$ & 2.1 & G0 (F7..G8) & $1.99(1.58 . .1 .99)$ \\
\hline $\mathrm{J} 3412344+2536562$ & $19.68 \pm 0.30$ & $17.94 \pm 0.18$ & $1.71 \pm 0.35$ & $1.27 \pm 0.20$ & $1.58 \pm 0.27$ & $1.99 \pm 0.26$ & 5.3 & & $0.15(0.15 . .1 .43)$ \\
\hline $\mathrm{J} 3412350+2540357$ & $18.54 \pm 0.27$ & $16.88 \pm 0.17$ & $1.52 \pm 0.32$ & $0.27 \pm 0.20$ & $0.91 \pm 0.21$ & $1.26 \pm 0.22$ & 24.6 & K7 (F7..) & 0.53( \\
\hline $\mathrm{J} 3412358+2544259$ & $18.13 \pm 0.27$ & $16.97 \pm 0.17$ & $0.73 \pm 0.32$ & $1.29 \pm 0.18$ & $1.74 \pm 0.18$ & & 5.1 & & $6.1 .68)$ \\
\hline $\mathrm{J} 3412359+2525549$ & $17.06 \pm 0.26$ & $16.57 \pm 0.17$ & $0.27 \pm 0.31$ & $0.45 \pm 0.18$ & $0.62 \pm 0.21$ & $0.71 \pm 0.23$ & 1.4 & F7 (F2..K0) & $0.82(0.15 . .1 .45)$ \\
\hline $\mathrm{J} 3412401+2546287$ & $17.78 \pm 0.26$ & $17.22 \pm 0.17$ & $0.38 \pm 0.31$ & $0.73 \pm 0.19$ & $0.82 \pm 0.25$ & $1.16 \pm 0.25$ & 3.2 & & $. .1 .45)$ \\
\hline $\mathrm{J} 3412418+2526538$ & $19.93 \pm 0.33$ & $19.25 \pm 0.28$ & $0.68 \pm 0.43$ & $2.36 \pm 0.32$ & $2.45 \pm$ & $3.03 \pm$ & 12.9 & M4 & .39) \\
\hline J3412431+2543125 & $17.11 \pm 0.26$ & $16.56 \pm 0.17$ & $0.38 \pm 0.31$ & $0.75 \pm 0.18$ & $1.01 \pm 0.19$ & .21 & 2.7 & K0 (F7..K0) & 48) \\
\hline J3412443+2532087 & $18.07 \pm 0.26$ & 17.13 & $0.70 \pm$ & 1. & 19 & 19 & 4.4 & & \\
\hline J3412457+2536086 & $15.39 \pm 0.26$ & $14.60 \pm 0.17$ & $0.35 \pm 0$ & $0.97 \pm$ & & & 2. & & 1.6 \\
\hline $\mathrm{J} 3412459+2553113$ & $19.27 \pm 0.27$ & $18.15=$ & $0.82=$ & 0.68 & 1.60 & 2.17 & 6.3 & M0 & 18) \\
\hline $\mathrm{J} 3412471+2538392$ & $15.38 \pm 0.26$ & 14.91 & $0.35 \pm 0.31$ & & & & 2. & 9..K1) & \\
\hline $\mathrm{J} 3412476+2$ & $17.22 \pm 0.26$ & 16.1 & $0.73=$ & 1.1 & 1.79 & 1.8 & 2.9 & M0 & \\
\hline J3412485+2540294 & $18.90 \pm 0.27$ & $18.44 \pm 0.19$ & $-0.06 \pm 0.33$ & 0.62 & & $2.16 \pm 0.24$ & 18.8 & K4 & 1.93 \\
\hline J3412487+2537468 & $18.62 \pm 0.27$ & 18.1 & $0.19 \pm 0.32$ & 1.0 & 1.5 & $2.22 \pm$ & 3.8 & $\mathrm{~K} 4$ & \\
\hline $\mathrm{J} 3412506+2527292$ & $15.52 \pm 0.26$ & $14.88 \pm 0.17$ & $0.32 \pm 0.31$ & $0.44 \pm 0.17$ & 0.7 & $0.82 \pm 0.18$ & 1.2 & G4 (F6..G9) & 1.18 \\
\hline $\mathrm{J} 3412510+2519346$ & $17.22 \pm 0.26$ & $16.70 \pm 0.17$ & $0.48 \pm 0.31$ & $0.87 \pm 0.19$ & $=0.19$ & & 1.9 & K4 & 0.15 \\
\hline $\mathrm{J} 3412513+2532395$ & $17.85 \pm 0.26$ & $17.26 \pm 0.17$ & $0.49 \pm 0.31$ & $0.76 \pm 0.20$ & $1.26 \pm 0.22$ & 1.3 & 1.1 & K3 & 46) \\
\hline $\mathrm{J} 3412532+2542487$ & $17.95 \pm 0.26$ & $17.00 \pm 0.17$ & $0.78 \pm 0.31$ & $0.74 \pm 0.20$ & \pm 0.22 & 1.70 & 3. & K7 & 14) \\
\hline $\mathrm{J} 3412542+2551032$ & $13.09 \pm 0.26$ & $12.48 \pm 0.17$ & $0.37 \pm 0.31$ & $0.61 \pm 0.17$ & 0.17 & $0.88 \pm 0.17$ & 2.0 & ..K3) & $. .1 .42)$ \\
\hline $\mathrm{J} 3412554+2545475$ & $18.44 \pm 0.27$ & $18.03 \pm 0.18$ & $0.20 \pm 0.32$ & $1.10 \pm 0.23$ & & \pm 0.30 & 4.5 & K4 & .47) \\
\hline J3412584+2528219 & $17.90 \pm 0.26$ & $17.29 \pm 0.17$ & $0.40 \pm 0.32$ & $0.68 \pm 0.20$ & $0.93 \pm 0.23$ & $1.47 \pm$ & 1.7 & K0 (F7..K5) & $0.80(0.01 . .1 .41)$ \\
\hline $\mathrm{J} 3412614+2519479$ & $17.26 \pm 0.26$ & $16.53 \pm 0.17$ & $0.37 \pm 0.31$ & $0.58 \pm 0.18$ & $0.94 \pm 0.19$ & 20 & 23 & G7 (G6. & .48) \\
\hline J3412616+2527186 & $14.79 \pm 0.26$ & $14.01 \pm 0.17$ & $0.50 \pm 0.31$ & $0.85 \pm 0.17$ & $1.37 \pm 0.17$ & 17 & 1.2 & K4 (K3..K6) & $1.02(0.66 . .1 .42)$ \\
\hline $7+2530447$ & $19.33 \pm 0.28$ & & $0.82 \pm 0.33$ & & & & 3. & & \\
\hline $\mathrm{J} 3412636+2527411$ & $14.20 \pm 0.26$ & $13.69 \pm 0.17$ & $0.28 \pm 0.31$ & 0.17 & $0.63 \pm 0.17$ & $0.67 \pm 0.17$ & 2. & F7 (F4..K0) & $0.89(0.02 . .1 .10)$ \\
\hline $\mathrm{J} 3412643+2543379$ & $13.36 \pm 0.26$ & & $0.39 \pm 0.31$ & & & 0.17 & 2. & & \\
\hline+2540106 & 18.24 & 17.69 & $1 \pm 0.32$ & & & & 3. & 1..K7) & $3.1 .35)$ \\
\hline++2536351 & & & & & & 8 & 2. & $.5 . \mathrm{M}$ & \\
\hline $\mathrm{J} 3412672+2535277$ & $14.35 \pm 0.26$ & $13.69 \pm 0.17$ & $0.36 \pm 0.31$ & $0.99 \pm 0.17$ & $1.43 \pm 0.17$ & $1.49=$ & 3. & K4 (K3..M0) & $1.13(0.01 . .1 .28)$ \\
\hline $\mathrm{J} 3412691+2550470$ & & & & & & & 1 & & \\
\hline $\mathrm{J} 3412701+2538577$ & $16.89 \pm 0.26$ & $16.34 \pm 0.17$ & $0.36 \pm 0.31$ & $0.99 \pm 0.17$ & $1.47 \pm 0.18$ & 1.47 & 3. & K4 (K0..K5) & $0.74(0.37 . .1 .47)$ \\
\hline $\mathrm{J} 3412702+2552473$ & $17.16 \pm 0.26$ & $16.14 \pm 0.17$ & $0.83 \pm 0.31$ & 0.18 & $=0.18$ & & 1.9 & K7 (1 & $0.71(0.06 . .1 .27)$ \\
\hline+2540395 & $17.86 \pm 0.26$ & 17 & $0.52 \pm 0.31$ & $-0.51 \pm 0.30$ & $0.69 \pm 0.26$ & 26 & 18.6 & G6 & 2..1.45) \\
\hline+2521035 & $6 \pm 0.26$ & & 1 & -0.0 & 7 & & 3 & Fo & $. .1 .30)$ \\
\hline $\mathrm{J} 3412752+2542128$ & $18.11 \pm 0.26$ & $17.29 \pm 0.17$ & $0.46 \pm 0.32$ & $0.88 \pm 0.19$ & $1.33 \pm 0.21$ & $1.52=$ & 1.5 & K4 (K4...M0) & $1.34(0.17 . .1 .34)$ \\
\hline $\mathrm{J} 3412791+2540356$ & $18.09 \pm 0.26$ & $1726+017$ & $0.67 \pm 0$ & & $=0.22$ & & 15.5 & M1) & $0.61(0.14 . .1 .36)$ \\
\hline $\mathrm{J} 3412792+2543392$ & $16.99 \pm 0.26$ & $16.32 \pm 0.17$ & $0.49 \pm 0.31$ & $0.76 \pm 0.18$ & $1.33 \pm 0.18$ & $1.25 \pm 0.19$ & 1.7 & K3 (K1..K7) & $0.67(0.04 . .0 .90)$ \\
\hline J3412794+2535091 & $17.06 \pm 0.26$ & $1655+017$ & $0.33 \pm 0.31$ & $0.94 \pm 0.18$ & $1.47 \pm 0.18$ & $1.53 \pm 0.19$ & 2.6 & K4 (K3..K5) & $0.68(0.36 . .1 .10)$ \\
\hline+2537207 & 0.26 & 0.17 & 31 & 17 & 18 & 17 & 2. & M1) & $.50)$ \\
\hline J3412796+2547469 & $17.57 \pm 0.26$ & 17.00 & $0.38 \pm 0.31$ & & & & 1.3 & & $0.75(0.10 . .1 .50)$ \\
\hline-2543064 & 0.27 & & $0.06 \pm 0.32$ & & & & 28.3 & 13) & ..1.49) \\
\hline $\mathrm{J} 341$ & & & & & & & 1. & & .1.44) \\
\hline J3412 & & & & & & & 4. & M2) & \\
\hline $\begin{array}{r}+2524174 \\
\end{array}$ & & & 0.3 & & & & 2. & G9 ( & 1.34 \\
\hline J341 & & & & & & & 2. & & \\
\hline+2549209 & 16 & & 0 & & 1. & $=0.17$ & 1. & K4 & 4..1.63) \\
\hline $\mathrm{J} 34128$ & & & 36 & & & & 2. & M4) & 0.6 \\
\hline $\mathrm{J} 34128$ & & & 0 & & & & 2. & G7 & 0.71( \\
\hline $\mathrm{J} 34128$ & & & & & & & 1. & & 0.4 \\
\hline $1+2540045$ & & $4+017$ & +031 & 18 & 0.19 & $=0.19$ & 2. & $\mathrm{~K}$ & $0.29(0.13 . .1 .32)$ \\
\hline & & & & & & & & & \\
\hline $\mathrm{J} 3412877+2529548$ & $18.89 \pm 0.27$ & $17.30 \pm 0.17$ & $1.22 \pm 0.32$ & $1.45 \pm 0.18$ & $2.05 \pm 0.18$ & $2.18 \pm 0.18$ & 2. & M3 (M3..M4) & $1.41(0.40 . .1 .41)$ \\
\hline J3412915+2531528 & & & & & & & 2.2 & K4 (G7..K6) & \\
\hline $\mathrm{J} 3412920+2550153$ & $16.78 \pm 0.26$ & $16.22 \pm 0.17$ & $0.42 \pm 0.31$ & \pm 0.18 & \pm 0.18 & .20 & 1.8 & 8...K3) & $0.53(0.11 . .1 .47)$ \\
\hline $1+2527575$ & & & & & & & 1. & M1 & \\
\hline $\mathrm{J} 3412928+2524299$ & $16.25 \pm 0.26$ & $15.64 \pm 0.17$ & $4 \pm 0.31$ & 0.9 & 1.3 & $1.36=$ & 2.4 & K4 & 0.29 \\
\hline & & & & & & & 1. & & \\
\hline $\mathrm{J} 3412934+2550408$ & $12.78 \pm 0.26$ & $12.22 \pm 0.17$ & $0.34 \pm 0.31$ & $0.61 \pm 0.17$ & $0.88 \pm 0.17$ & 0.89 & 1.9 & G7 (F7..K1) & 0.85 \\
\hline 34535 & & & & & & & 2.2 & K4 (G7..K4) & $0.12(0.12 . .1 .43)$ \\
\hline $\mathrm{J} 3412944+2536236$ & $14.75 \pm 0.26$ & $14.11 \pm 0.17$ & $0.42 \pm 0.31$ & \pm 0.17 & $1.41 \pm 0.17$ & $1.46 \pm$ & 2.2 & K4 (K1..K5) & $0.81(0.57$. \\
\hline & & & & & & & 1.3 & G7 (F7..K3) & $0.87(0.03 . .1 .49)$ \\
\hline $\mathrm{J} 3413004+2522119$ & $12.94 \pm 0.26$ & $12.26 \pm 0.17$ & $0.29 \pm 0.31$ & $0.47 \pm 0.17$ & $0.64 \pm 0.17$ & $0.66 \pm 0.17$ & 1.6 & F7 (F7..K2) & $1.46(0.26 . .1 .46)$ \\
\hline $\mathrm{J} 3413009+2526187$ & $18.32 \pm 0.27$ & $17.23 \pm 0.17$ & $0.65 \pm 0.32$ & $0.18 \pm 0.21$ & $0.40 \pm 0.27$ & & 3.4 & G0 (F5..G9) & $1.65(0.75 . .1 .65)$ \\
\hline
\end{tabular}




\begin{tabular}{|c|c|c|c|c|c|c|c|c|c|}
\hline \multirow[b]{2}{*}{ 2MASS-ID } & \multicolumn{2}{|c|}{ measured magnitudes } & & extinction c & ected colors & & & & \\
\hline & Rmag & Imag & R-I & I-J & $\mathrm{I}-\mathrm{H}$ & $\mathrm{I}-\mathrm{K}_{s}$ & $\chi^{2}$ & SpT & $\mathrm{Av}$ \\
\hline $\mathrm{J} 3413018+2542541$ & $20.14 \pm 0.34$ & $18.36 \pm 0.19$ & $1.52 \pm 0.39$ & $1.50 \pm 0.22$ & $2.45 \pm 0.22$ & $2.19 \pm 0.27$ & 5.3 & M4 (M3..M5) & $1.00(0.15 . .1 .42)$ \\
\hline $\mathrm{J} 3413047+2551210$ & $15.83 \pm 0.26$ & $15.17 \pm 0.17$ & $0.27 \pm 0.31$ & $0.03 \pm 0.17$ & $0.31 \pm 0.18$ & $0.31 \pm 0.18$ & 1.6 & F1 (A7..G5) & $1.45(0.30 . .1 .48)$ \\
\hline $\mathrm{J} 3413055+2537462$ & $18.14 \pm 0.26$ & $17.33 \pm 0.17$ & $0.58 \pm 0.32$ & $1.19 \pm 0.18$ & $1.87 \pm 0.19$ & $1.97 \pm 0.19$ & 5.5 & M0 (K5..M2) & $0.89(0.08 . .1 .50)$ \\
\hline $\mathrm{J} 3413066+2529356$ & $14.92 \pm 0.26$ & $14.33 \pm 0.17$ & $0.38 \pm 0.31$ & $0.70 \pm 0.17$ & $1.02 \pm 0.17$ & $1.06 \pm 0.17$ & 1.8 & K0 (G4..K3) & $0.80(0.29 . .1 .33)$ \\
\hline $\mathrm{J} 3413102+2551235$ & $15.90 \pm 0.26$ & $15.21 \pm 0.17$ & $0.40 \pm 0.31$ & $-0.15 \pm 0.17$ & $0.25 \pm 0.18$ & $0.23 \pm 0.19$ & 4.5 & F0 (A5..F8) & $1.10(0.41 . .1 .34)$ \\
\hline $\mathrm{J} 3413112+2534154$ & $16.42 \pm 0.26$ & $15.53 \pm 0.17$ & $0.64 \pm 0.31$ & $1.17 \pm 0.17$ & $1.83 \pm 0.17$ & $1.92 \pm 0.17$ & 4.3 & M0 (M0..M2) & $0.96(0.48 . .1 .15)$ \\
\hline $\mathrm{J} 3413123+2527567$ & $19.19 \pm 0.28$ & $17.52 \pm 0.17$ & $1.50 \pm 0.33$ & $0.86 \pm 0.22$ & $1.46 \pm 0.24$ & $1.90 \pm 0.24$ & 5.9 & M2 (M0..M3) & $0.64(0.34 . .1 .43)$ \\
\hline $\mathrm{J} 3413134+2520252$ & $20.92 \pm 0.69$ & $18.89 \pm 0.23$ & $1.74 \pm 0.73$ & $1.34 \pm 0.32$ & $3.09 \pm \ldots$ & $2.97 \pm 0.26$ & 4.5 & M5 (M4..M6) & $1.11(0.46 . .1 .46)$ \\
\hline $\mathrm{J} 3413164+2521216$ & $16.98 \pm 0.26$ & $16.25 \pm 0.17$ & $0.48 \pm 0.31$ & $0.90 \pm 0.18$ & $1.37 \pm 0.18$ & $1.44 \pm 0.19$ & 1.5 & $\mathrm{~K} 4$ (K2..K6) & $0.94(0.55 . .1 .46)$ \\
\hline $\mathrm{J} 3413191+2530576$ & $18.12 \pm 0.26$ & $17.18 \pm 0.17$ & $0.79 \pm 0.32$ & $1.05 \pm 0.19$ & $1.64 \pm 0.20$ & $1.99 \pm 0.21$ & 2.0 & M0 (K5..M1) & $0.58(0.26 . .1 .49)$ \\
\hline $\mathrm{J} 3413197+2523464$ & $17.13 \pm 0.26$ & $16.47 \pm 0.17$ & $0.49 \pm 0.31$ & $0.86 \pm 0.18$ & $1.29 \pm 0.19$ & $1.20 \pm 0.20$ & 2.7 & K3 (G7..K7) & $0.63(0.12 . .1 .35)$ \\
\hline $\mathrm{J} 3413205+2541526$ & $18.28 \pm 0.27$ & $17.25 \pm 0.17$ & $1.00 \pm 0.32$ & $-0.06 \pm 0.27$ & $1.09 \pm 0.26$ & $1.85 \pm 0.22$ & 20.3 & K7 (F8..M0) & $0.09(0.09 . .1 .43)$ \\
\hline $\mathrm{J} 3413278+2552223$ & $17.85 \pm 0.26$ & $16.97 \pm 0.17$ & $0.51 \pm 0.31$ & $-0.04 \pm 0.24$ & $0.58 \pm 0.24$ & $1.12 \pm \ldots$ & 4.0 & F6 (F3..G9) & $1.40(0.40 . .1 .46)$ \\
\hline $\mathrm{J} 3413280+2536590$ & $18.67 \pm 0.27$ & $17.88 \pm 0.18$ & $0.42 \pm 0.33$ & $1.08 \pm 0.22$ & $1.82 \pm 0.22$ & $2.33 \pm 0.22$ & 11.3 & M0 (M0..M3) & $1.41(0.41 . .1 .44)$ \\
\hline $\mathrm{J} 3413325+2535192$ & $18.59 \pm 0.27$ & $17.25 \pm 0.17$ & $1.16 \pm 0.32$ & $1.42 \pm 0.18$ & $2.07 \pm 0.19$ & $2.26 \pm 0.19$ & 3.0 & M3 (M1..M4) & $0.68(0.01 . .1 .48)$ \\
\hline $\mathrm{J} 3413360+2531428$ & $14.75 \pm 0.26$ & $14.21 \pm 0.17$ & $0.53 \pm 0.31$ & $0.94 \pm 0.17$ & $1.32 \pm 0.17$ & $1.41 \pm 0.17$ & 2.5 & K4 (G5..K5) & $0.07(0.07 . .1 .48)$ \\
\hline $\mathrm{J} 3413362+2546037$ & $19.20 \pm 0.28$ & $18.35 \pm 0.19$ & $0.61 \pm 0.33$ & $1.26 \pm 0.27$ & $1.66 \pm 0.31$ & $2.15 \pm 0.31$ & 4.3 & M0 (K5..M2) & $0.93(0.33 . .1 .30)$ \\
\hline $\mathrm{J} 3413377+2522302$ & $16.58 \pm 0.26$ & $15.84 \pm 0.17$ & $0.38 \pm 0.31$ & $0.47 \pm 0.17$ & $0.61 \pm 0.19$ & $0.79 \pm 0.19$ & 1.8 & G4 (F7..K0) & $1.36(0.75 . .1 .47)$ \\
\hline $\mathrm{J} 3413391+2521440$ & $16.86 \pm 0.26$ & $16.12 \pm 0.17$ & $0.42 \pm 0.31$ & $0.69 \pm 0.18$ & $1.01 \pm 0.18$ & $1.01 \pm 0.19$ & 2.0 & $\mathrm{~K} 0(\mathrm{G} 8 . . \mathrm{K} 7)$ & $1.20(0.02 . .1 .39)$ \\
\hline J3413394+2532097 & $17.25 \pm 0.26$ & $16.10 \pm 0.17$ & $0.68 \pm 0.31$ & $1.24 \pm 0.17$ & $1.75 \pm 0.17$ & $1.89 \pm 0.17$ & 4.5 & M0 (M0..M3) & $1.77(0.25 . .1 .77)$ \\
\hline $\mathrm{J} 3413402+2543092$ & $17.49 \pm 0.26$ & $16.60 \pm 0.17$ & $0.61 \pm 0.31$ & $0.71 \pm 0.19$ & $1.24 \pm 0.20$ & $1.68 \pm 0.20$ & 3.3 & K5 (K4..M0) & $1.03(0.20 . .1 .32)$ \\
\hline $\mathrm{J} 3413411+2530549$ & $18.15 \pm 0.26$ & $17.21 \pm 0.17$ & $0.50 \pm 0.32$ & $0.67 \pm 0.20$ & $0.90 \pm 0.23$ & $0.95 \pm 0.28$ & 2.5 & $\mathrm{~K} 0$ (K0..K7) & $1.64(0.19 . .1 .64)$ \\
\hline $\mathrm{J} 3413432+2542294$ & $17.97 \pm 0.26$ & $17.60 \pm 0.17$ & $0.13 \pm 0.32$ & $1.06 \pm 0.21$ & $1.61 \pm 0.22$ & $1.27 \pm \ldots$ & 5.4 & K4 (K2..M0) & $0.90(0.14 . .1 .42)$ \\
\hline $\mathrm{J} 3413443+2526306$ & $14.80 \pm 0.26$ & $14.03 \pm 0.17$ & $0.51 \pm 0.31$ & $0.83 \pm 0.17$ & $1.21 \pm 0.17$ & $1.29 \pm 0.17$ & 1.9 & K3 (K0..K6) & $0.98(0.26 . .1 .46)$ \\
\hline $\mathrm{J} 3413459+2549314$ & $18.59 \pm 0.27$ & $18.69 \pm 0.20$ & $-0.63 \pm 0.33$ & $1.19 \pm 0.30$ & $2.06 \pm 0.28$ & $2.47 \pm 0.27$ & 40.0 & $\mathrm{~K} 4$ (K1..M1) & $1.97(0.28 . .1 .97)$ \\
\hline $\mathrm{J} 3413462+2541181$ & $19.25 \pm 0.28$ & $17.50 \pm 0.17$ & $1.68 \pm 0.33$ & $1.60 \pm 0.19$ & $2.09 \pm 0.20$ & $2.29 \pm 0.21$ & 1.7 & M4 (M3..M4) & $0.25(0.01 . .1 .16)$ \\
\hline $\mathrm{J} 3413492+2551060$ & $17.86 \pm 0.27$ & $16.97 \pm 0.17$ & $0.71 \pm 0.32$ & $0.76 \pm 0.19$ & $1.83 \pm \ldots$ & $1.67 \pm \ldots$ & 1.0 & K6 (K1..M0) & $0.68(0.07 . .1 .49)$ \\
\hline $\mathrm{J} 3413503+2521235$ & $17.44 \pm 0.26$ & $16.54 \pm 0.17$ & $0.49 \pm 0.31$ & $0.68 \pm 0.18$ & $1.02 \pm 0.19$ & $0.90 \pm 0.21$ & 3.1 & K0 (G7..K7) & $1.55(0.24 . .1 .55)$ \\
\hline $\mathrm{J} 3413530+2541064$ & $18.15 \pm 0.26$ & $17.35 \pm 0.17$ & $0.48 \pm 0.32$ & $0.88 \pm 0.20$ & $1.62 \pm \ldots$ & $1.43 \pm 0.25$ & 1.3 & K4 (K3..M0) & $1.19(0.30 . .1 .39)$ \\
\hline $\mathrm{J} 3413535+2547395$ & $17.81 \pm 0.26$ & $17.13 \pm 0.17$ & $0.48 \pm 0.32$ & $0.81 \pm 0.20$ & $1.32 \pm 0.21$ & $1.19 \pm 0.27$ & 2.0 & K3 (K0..K7) & $0.74(0.17 . .1 .42)$ \\
\hline $\mathrm{J} 3413536+2523222$ & $18.01 \pm 0.27$ & $17.45 \pm 0.17$ & $0.38 \pm 0.32$ & $0.90 \pm 0.22$ & $1.07 \pm \ldots$ & $1.61 \pm$ & 1.3 & K4 (K1..K6) & $0.69(0.14 . .1 .26)$ \\
\hline $\mathrm{J} 3413539+2539176$ & $17.88 \pm 0.26$ & $16.92 \pm 0.17$ & $0.83 \pm 0.31$ & $1.06 \pm 0.18$ & $1.53 \pm 0.20$ & $2.02 \pm 0.20$ & 3.3 & M0 (K4..M2) & $0.52(0.12 . .1 .43)$ \\
\hline $\mathrm{J} 3413548+2553253$ & $19.59 \pm 0.27$ & $17.71 \pm 0.17$ & $1.78 \pm 0.32$ & $1.35 \pm 0.20$ & $2.16 \pm 0.20$ & $2.25 \pm 0.23$ & 2.8 & M4 (M3..M4) & $0.38(0.38 . .1 .49)$ \\
\hline $\mathrm{J} 3413578+2533530$ & $18.81 \pm 0.27$ & $17.70 \pm 0.17$ & $0.87 \pm 0.32$ & $0.75 \pm 0.25$ & $1.32 \pm 0.26$ & $1.48 \pm 0.31$ & 1.5 & K7 (K3..K7) & $0.92(0.65 . .1 .43)$ \\
\hline $\mathrm{J} 3413581+2551599$ & $15.59 \pm 0.26$ & $14.99 \pm 0.17$ & $0.33 \pm 0.31$ & $0.61 \pm 0.17$ & $0.91 \pm 0.17$ & $0.89 \pm 0.17$ & 1.9 & G7 (F7..K2) & $1.03(0.35 . .1 .48)$ \\
\hline $\mathrm{J} 3413582+2538194$ & $16.37 \pm 0.26$ & $15.82 \pm 0.17$ & $0.51 \pm 0.31$ & $1.04 \pm 0.17$ & $1.26 \pm 0.18$ & $1.42 \pm 0.18$ & 4.5 & K4 (G7..K4) & $0.18(0.13 . .1 .49)$ \\
\hline $\mathrm{J} 3413585+2524209$ & $15.50 \pm 0.26$ & $14.87 \pm 0.17$ & $0.33 \pm 0.31$ & $0.46 \pm 0.17$ & $0.75 \pm 0.17$ & $0.75 \pm 0.18$ & 1.3 & G4 (F9..K2) & $1.15(0.13 . .1 .49)$ \\
\hline $\mathrm{J} 3413597+2522230$ & $17.39 \pm 0.26$ & $16.17 \pm 0.17$ & $1.21 \pm 0.31$ & $1.07 \pm 0.17$ & $1.75 \pm 0.18$ & $1.94 \pm 0.18$ & 1.1 & M2 (K6..M2) & $0.04(0.04 . .1 .39)$ \\
\hline $\mathrm{J} 3413645+2550269$ & $13.76 \pm 0.26$ & $13.06 \pm 0.17$ & $0.54 \pm 0.31$ & $0.89 \pm 0.17$ & $1.33 \pm 0.17$ & $1.42 \pm 0.17$ & 1.8 & $\mathrm{~K} 4$ (K3..K7) & $0.63(0.21 . .1 .18)$ \\
\hline $\mathrm{J} 3413648+2528034$ & $17.16 \pm 0.26$ & $15.88 \pm 0.17$ & $1.11 \pm 0.31$ & $1.14 \pm 0.17$ & $1.84 \pm 0.18$ & $1.95 \pm 0.18$ & 1.4 & M2 (M0..M2) & $0.63(0.38 . .1 .14)$ \\
\hline $\mathrm{J} 3413649+2548008$ & $17.14 \pm 0.26$ & $16.39 \pm 0.17$ & $0.49 \pm 0.31$ & $0.83 \pm 0.18$ & $1.18 \pm 0.18$ & $1.33 \pm 0.19$ & 1.9 & $\mathrm{~K} 3$ (K2..K7) & $0.97(0.16 . .1 .26)$ \\
\hline $\mathrm{J} 3413669+2541597$ & $15.21 \pm 0.26$ & $14.39 \pm 0.17$ & $0.44 \pm 0.31$ & $0.83 \pm 0.17$ & $1.42 \pm 0.17$ & $1.49 \pm 0.17$ & 1.2 & K4 (K4..M0) & $1.38(0.35 . .1 .38)$ \\
\hline $\mathrm{J} 3413708+2541518$ & $15.42 \pm 0.26$ & $14.72 \pm 0.17$ & $0.56 \pm 0.31$ & $-0.24 \pm 0.17$ & $0.17 \pm 0.18$ & $0.15 \pm 0.18$ & 8.8 & F0 (A2..F5) & $0.53(0.15 . .1 .15)$ \\
\hline $\mathrm{J} 3413735+2537325$ & $16.17 \pm 0.26$ & $15.31 \pm 0.17$ & $0.40 \pm 0.31$ & $0.91 \pm 0.17$ & $1.46 \pm 0.17$ & $1.46 \pm 0.17$ & 1.9 & K4 (K4..M0) & $1.69(0.38 . .1 .69)$ \\
\hline $\mathrm{J} 3413738+2544596$ & $20.17 \pm 0.35$ & $18.79 \pm 0.19$ & $0.95 \pm 0.40$ & $1.49 \pm 0.26$ & $2.11 \pm 0.28$ & $2.51 \pm 0.30$ & 4.9 & M3 (M2..M5) & $1.61(0.16 . .1 .62)$ \\
\hline $\mathrm{J} 3413769+2521351$ & $16.07 \pm 0.26$ & $15.26 \pm 0.17$ & $0.60 \pm 0.31$ & $-0.02 \pm 0.18$ & $0.11 \pm 0.18$ & $-0.05 \pm 0.21$ & 7.6 & F0 (A6..G2) & $0.81(0.08 . .1 .08)$ \\
\hline $\mathrm{J} 3413791+2518595$ & $18.57 \pm 0.27$ & $17.73 \pm 0.18$ & $0.83 \pm 0.32$ & $2.53 \pm 0.18$ & $2.97 \pm 0.19$ & $2.99 \pm 0.20$ & 52.7 & M5 (M1..M6) & $0.00(0.00 . .1 .31)$ \\
\hline $\mathrm{J} 3413819+2543022$ & $12.97 \pm 0.26$ & $12.35 \pm 0.17$ & $0.37 \pm 0.31$ & $0.99 \pm 0.17$ & $1.43 \pm 0.17$ & $1.47 \pm 0.17$ & 3.0 & $\mathrm{~K} 4$ (K2..K6) & $0.92(0.58 . .1 .43)$ \\
\hline $\mathrm{J} 3413831+2551110$ & $18.16 \pm 0.27$ & $16.98 \pm 0.17$ & $0.85 \pm 0.32$ & $1.14 \pm 0.18$ & $1.84 \pm 0.18$ & $1.64 \pm 0.20$ & 4.7 & M0 (M0..M3) & $1.23(0.15 . .1 .41)$ \\
\hline $\mathrm{J} 3413834+2545241$ & $19.76 \pm 0.31$ & $17.97 \pm 0.18$ & $1.78 \pm 0.35$ & $1.89 \pm 0.20$ & $2.40 \pm 0.21$ & $2.80 \pm 0.23$ & 1.7 & M5 (M4..M5) & $0.04(0.04 . .1 .31)$ \\
\hline $\mathrm{J} 3413865+2543251$ & $17.24 \pm 0.26$ & $16.63 \pm 0.17$ & $0.22 \pm 0.31$ & $0.42 \pm 0.19$ & $0.53 \pm 0.21$ & $0.48 \pm 0.28$ & 1.8 & $\mathrm{~F} 5(\mathrm{~F} 3 . . \mathrm{K} 1)$ & $1.45(0.32 . .1 .50)$ \\
\hline $\mathrm{J} 3413868+2540177$ & $18.72 \pm 0.27$ & $17.09 \pm 0.17$ & $1.48 \pm 0.32$ & $1.31 \pm 0.18$ & $2.02 \pm 0.19$ & $1.94 \pm 0.20$ & 3.8 & M3 (M1..M3) & $0.59(0.20 . .1 .47)$ \\
\hline $\mathrm{J} 3413887+2526365$ & $16.18 \pm 0.26$ & $15.23 \pm 0.17$ & $0.84 \pm 0.31$ & $1.08 \pm 0.17$ & $1.69 \pm 0.17$ & $1.84 \pm 0.17$ & 1.4 & M0 (K4..M1) & $0.38(0.26 . .1 .40)$ \\
\hline $\mathrm{J} 3413911+2553287$ & $16.31 \pm 0.26$ & $15.26 \pm 0.17$ & $0.85 \pm 0.31$ & $1.05 \pm 0.17$ & $1.75 \pm 0.17$ & $1.80 \pm 0.17$ & 1.5 & M0 (K6..M2) & $0.75(0.27 . .1 .48)$ \\
\hline $\mathrm{J} 3413922+2547295$ & $12.16 \pm 0.26$ & $11.66 \pm 0.17$ & $0.41 \pm 0.31$ & $0.71 \pm 0.17$ & $0.97 \pm 0.17$ & $1.06 \pm 0.17$ & 2.2 & $\mathrm{~K} 0$ (F6..K2) & $0.36(0.04 . .1 .50)$ \\
\hline $\mathrm{J} 3413926+2531221$ & $15.36 \pm 0.26$ & $14.80 \pm 0.17$ & $0.34 \pm 0.31$ & $0.77 \pm 0.17$ & $0.99 \pm 0.17$ & $1.08 \pm 0.17$ & 2.8 & $\mathrm{~K} 0(\mathrm{G} 1 . . \mathrm{K} 4)$ & $0.80(0.12 . .1 .47)$ \\
\hline $\mathrm{J} 3413953+2546405$ & $16.32 \pm 0.26$ & $15.50 \pm 0.17$ & $0.37 \pm 0.31$ & $-1.05 \pm 0.20$ & $-0.77 \pm 0.23$ & $-0.02 \pm 0.21$ & 35.4 & B6 (B0..F7) & $1.68(0.16 . .1 .68)$ \\
\hline $\mathrm{J} 3414011+2533501$ & $18.92 \pm 0.27$ & $17.97 \pm 0.17$ & $0.70 \pm 0.32$ & $1.15 \pm 0.22$ & $1.43 \pm$ & $0.40 \pm$ & 1.9 & M0 (K5..M2) & $0.93(0.22 . .1 .47)$ \\
\hline $\mathrm{J} 3414019+2537341$ & $16.46 \pm 0.26$ & $16.03 \pm 0.17$ & $0.32 \pm 0.31$ & $0.81 \pm 0.18$ & $1.00 \pm 0.19$ & $1.07 \pm 0.20$ & 3.6 & K0 (F6..K2) & $0.40(0.16 . .1 .46)$ \\
\hline $\mathrm{J} 3414034+2545420$ & $17.68 \pm 0.26$ & $17.36 \pm 0.17$ & $0.32 \pm 0.31$ & $1.05 \pm 0.20$ & $1.17 \pm 0.27$ & $1.14 \pm$. & 4.9 & K4 (G0..K4) & $0.00(0.00 . .1 .46)$ \\
\hline $\mathrm{J} 3414046+2543285$ & $18.42 \pm 0.27$ & $18.19 \pm 0.18$ & $-0.13 \pm 0.32$ & $1.36 \pm 0.23$ & $1.54 \pm 0.28$ & $1.98 \pm 0.30$ & 16.1 & $\mathrm{~K} 4$ (K1..M2) & $1.34(0.31 . .1 .50)$ \\
\hline $\mathrm{J} 3414050+2544275$ & $18.89 \pm 0.27$ & $18.18 \pm 0.18$ & $0.26 \pm 0.33$ & $0.94 \pm 0.27$ & $1.46 \pm 0.28$ & $1.73 \pm 0.29$ & 2.9 & K4 (K3..M0) & $1.69(0.23 . .1 .69)$ \\
\hline $\mathrm{J} 3414059+2541308$ & $16.60 \pm 0.26$ & $15.63 \pm 0.17$ & $0.75 \pm 0.31$ & $-0.25 \pm 0.18$ & $-0.03 \pm 0.19$ & $0.04 \pm 0.23$ & 13.3 & F0 (A1..G4) & $0.86(0.10 . .1 .23)$ \\
\hline $\mathrm{J} 3414080+2541119$ & $16.88 \pm 0.26$ & $15.68 \pm 0.17$ & $0.78 \pm 0.31$ & $1.21 \pm 0.17$ & $1.70 \pm 0.17$ & $1.82 \pm 0.18$ & 3.3 & M0 (M0..M3) & $1.58(0.42 . .1 .58)$ \\
\hline $\mathrm{J} 3414101+2533377$ & $18.21 \pm 0.26$ & $17.73 \pm 0.17$ & $0.35 \pm 0.32$ & $0.77 \pm 0.25$ & $1.26 \pm 0.29$ & $1.87 \pm 0.29$ & 4.7 & K4 (G6..K7) & $0.45(0.19 . .1 .44)$ \\
\hline $\mathrm{J} 3414120+2546359$ & $18.75 \pm 0.27$ & $17.79 \pm 0.18$ & $0.62 \pm 0.32$ & $1.14 \pm \ldots$ & $1.95 \pm$ & $2.04 \pm 0.25$ & 2.3 & M0 (M0..M3) & $1.27(0.12 . .1 .46)$ \\
\hline $\mathrm{J} 3414130+2536478$ & $18.22 \pm 0.27$ & $18.30 \pm 0.19$ & $-0.35 \pm 0.32$ & $1.46 \pm 0.23$ & $1.65 \pm 0.29$ & $2.21 \pm 0.29$ & 26.6 & K4 (G4..M1) & $1.01(0.18 . .1 .47)$ \\
\hline $\mathrm{J} 3414136+2542498$ & $18.93 \pm 0.27$ & $18.20 \pm 0.18$ & $0.19 \pm 0.33$ & $1.07 \pm 0.24$ & $1.42 \pm$ & $0.82 \pm$ & 3.2 & K4 (K3..M2) & $2.01(0.21 . .2 .01)$ \\
\hline $\mathrm{J} 3414151+2522117$ & $8.04 \pm 0.26$ & $7.33 \pm 0.17$ & $0.32 \pm 0.31$ & $1.53 \pm 0.17$ & $1.85 \pm 0.17$ & $2.08 \pm 0.17$ & 20.9 & M0 (M0..M4) & $1.49(0.01 . .1 .49)$ \\
\hline $\mathrm{J} 3414180+2532418$ & $18.85 \pm 0.27$ & $17.64 \pm 0.17$ & $0.82 \pm 0.32$ & $1.18 \pm 0.20$ & $1.68 \pm 0.21$ & $1.81 \pm 0.23$ & 2.4 & M0 (M0..M3) & $1.46(0.25 . .1 .46)$ \\
\hline $\mathrm{J} 3414196+2534298$ & $17.56 \pm 0.26$ & $16.99 \pm 0.17$ & $0.31 \pm 0.31$ & $0.83 \pm 0.19$ & $0.78 \pm 0.23$ & $1.25 \pm 0.24$ & 5.8 & $\mathrm{~K} 0$ (G2..K6) & $0.98(0.04 . .1 .30)$ \\
\hline $\mathrm{J} 3414200+2546503$ & $16.05 \pm 0.26$ & $15.40 \pm 0.17$ & $0.45 \pm 0.31$ & $0.96 \pm 0.17$ & $1.40 \pm 0.17$ & $1.42 \pm 0.18$ & 2.4 & $\mathrm{~K} 4$ (K1..K6) & $0.76(0.41 . .1 .29)$ \\
\hline $\mathrm{J} 3414221+2531563$ & $17.99 \pm 0.26$ & $16.76 \pm 0.17$ & $0.85 \pm 0.31$ & $1.24 \pm 0.18$ & $1.75 \pm 0.18$ & $1.97 \pm 0.18$ & 3.1 & M1 (M0..M3) & $1.41(0.31 . .1 .50)$ \\
\hline $\mathrm{J} 3414223+2524360$ & $15.77 \pm 0.26$ & $15.05 \pm 0.17$ & $0.39 \pm 0.31$ & $0.67 \pm 0.17$ & $1.03 \pm 0.17$ & $1.05 \pm 0.17$ & 1.6 & $\mathrm{~K} 0$ (G7..K7) & $1.24(0.10 . .1 .50)$ \\
\hline $\mathrm{J} 3414239+2538025$ & $17.19 \pm 0.26$ & $16.44 \pm 0.17$ & $0.39 \pm 0.31$ & $0.93 \pm 0.18$ & $1.37 \pm 0.18$ & $1.55 \pm 0.18$ & 2.0 & K4 (K3..M0) & $1.37(0.29 . .1 .47)$ \\
\hline $\mathrm{J} 3414241+2538247$ & $15.04 \pm 0.26$ & $14.24 \pm 0.17$ & $0.51 \pm 0.31$ & $0.70 \pm 0.18$ & $1.24 \pm 0.18$ & $1.35 \pm 0.18$ & 1.0 & K3 (K0..K7) & $1.10(0.39 . .1 .47)$ \\
\hline $\mathrm{J} 3414249+2527006$ & $20.02 \pm 0.34$ & $20.44 \pm 0.58$ & $-0.79 \pm 0.67$ & $2.91 \pm 0.63$ & $3.84 \pm 0.62$ & $4.19 \pm 0.62$ & 40.1 & M3 (K5..M5) & $1.38(0.38 . .1 .50)$ \\
\hline $\mathrm{J} 3414254+2536202$ & $18.03 \pm 0.26$ & $16.89 \pm 0.17$ & $0.82 \pm 0.31$ & $1.09 \pm 0.18$ & $1.75 \pm 0.18$ & $1.81 \pm 0.19$ & 1.7 & M0 (M0..M2) & $1.22(0.60 . .1 .44)$ \\
\hline $\mathrm{J} 3414256+2527379$ & $17.99 \pm 0.26$ & $17.21 \pm 0.17$ & $0.39 \pm 0.32$ & $0.35 \pm 0.22$ & $0.88 \pm 0.23$ & $1.09 \pm 0.26$ & 1.8 & G9 (G7..K5) & $1.47(0.28 . .1 .47)$ \\
\hline $\mathrm{J} 3414339+2526442$ & $19.38 \pm 0.29$ & $19.11 \pm 0.23$ & $-0.32 \pm 0.37$ & $1.78 \pm 0.29$ & $2.58 \pm 0.27$ & $2.66 \pm \ldots$ & 32.5 & M0 (K0..M5) & $2.20(0.16 . .2 .27)$ \\
\hline $\mathrm{J} 3414342+2544157$ & $16.16 \pm 0.26$ & $15.44 \pm 0.17$ & $0.45 \pm 0.31$ & $-1.25 \pm 0.22$ & $-0.61 \pm 0.23$ & $0.19 \pm$ & 22.1 & B0 (B0..A1) & $1.03(0.01 . .1 .33)$ \\
\hline $\mathrm{J} 3414374+2532414$ & $19.38 \pm 0.28$ & $18.13 \pm 0.18$ & $0.83 \pm 0.33$ & $1.37 \pm 0.22$ & $1.90 \pm 0.22$ & $2.17 \pm 0.24$ & 5.2 & M2 (M2..M4) & $1.58(0.26 . .1 .58)$ \\
\hline
\end{tabular}




\begin{tabular}{|c|c|c|c|c|c|c|c|c|c|}
\hline \multirow[b]{2}{*}{ 2MASS-ID } & \multicolumn{2}{|c|}{ measured magnitudes } & & extinction c & ected colors & & & & \\
\hline & Rmag & Imag & R-I & I-J & $\mathrm{I}-\mathrm{H}$ & $\mathrm{I}-\mathrm{K}_{s}$ & 2 & SpT & $\mathrm{Av}$ \\
\hline J3414387+2543233 & $18.05 \pm 0.26$ & $17.40 \pm 0.17$ & $0.33 \pm 0.32$ & $0.41 \pm 0.25$ & $1.30 \pm 0.23$ & $1.97 \pm 0.21$ & 14.1 & K4 (K2..M2) & $1.18(0.02 . .1 .40)$ \\
\hline J3414397+2545469 & $17.97 \pm 0.26$ & $17.35 \pm 0.17$ & $0.39 \pm 0.32$ & $1.08 \pm 0.19$ & $1.40 \pm 0.22$ & $1.39 \pm 0.27$ & 4.1 & K4 (K1..K6) & $0.86(0.35 . .1 .36)$ \\
\hline $\mathrm{J} 3414417+2528193$ & $21.73 \pm 0.96$ & $17.94 \pm 0.18$ & $3.74 \pm 0.98$ & $1.95 \pm 0.19$ & $2.46 \pm 0.20$ & $2.83 \pm 0.21$ & 10.2 & M6 (M4..M6) & $0.14(0.11 . .1 .34)$ \\
\hline $\mathrm{J} 3414421+2535543$ & $19.85 \pm 0.31$ & $19.07 \pm 0.21$ & $0.33 \pm 0.38$ & $1.64 \pm 0.30$ & $2.66 \pm 0.27$ & $2.91 \pm 0.29$ & 28.6 & M3 (M1..M5) & $1.68(0.06 . .1 .68)$ \\
\hline $\mathrm{J} 3414425+2547408$ & $16.54 \pm 0.26$ & $15.79 \pm 0.17$ & $0.42 \pm 0.31$ & $0.97 \pm 0.17$ & $1.44 \pm 0.17$ & $1.42 \pm 0.18$ & 2.7 & K4 (K3...M1) & $1.24(0.05 . .1 .43)$ \\
\hline $\mathrm{J} 3414436+2532027$ & $19.04 \pm 0.27$ & $17.38 \pm 0.17$ & $1.43 \pm 0.32$ & $1.64 \pm 0.18$ & $2.27 \pm 0.18$ & $2.45 \pm 0.19$ & 3.0 & M4 (M3..M5) & $0.88(0.24 . .1 .40)$ \\
\hline J3414454+2534545 & $17.15 \pm 0.26$ & $16.36 \pm 0.17$ & $0.70 \pm 0.31$ & $0.65 \pm 0.19$ & $1.31 \pm 0.20$ & $1.84 \pm 0.20$ & 5.2 & K7 (G8..K7) & $0.34(0.21 . .1 .48)$ \\
\hline $\mathrm{J} 3414471+2550276$ & $17.10 \pm 0.26$ & $16.39 \pm 0.17$ & $0.50 \pm 0.31$ & $0.88 \pm 0.18$ & $1.36 \pm 0.18$ & $1.45 \pm 0.19$ & 1.3 & K4 (K1..K6) & $0.79(0.58 . .1 .39)$ \\
\hline $\mathrm{J} 3414478+2541383$ & $15.96 \pm 0.26$ & $15.39 \pm 0.17$ & $0.47 \pm 0.31$ & $0.92 \pm 0.17$ & $1.38 \pm 0.17$ & $1.44 \pm 0.17$ & 1.8 & K4 (K1..K6) & $0.36(0.04 . .0 .99)$ \\
\hline $\mathrm{J} 3414483+2529175$ & $17.73 \pm 0.26$ & $16.69 \pm 0.17$ & $0.58 \pm 0.31$ & $1.29 \pm 0.18$ & $1.82 \pm 0.18$ & $1.93 \pm 0.18$ & 6.7 & M0 (M0..M3) & $1.74(0.32 . .1 .74)$ \\
\hline J3414499+2520466 & $16.63 \pm 0.26$ & $15.72 \pm 0.17$ & $0.47 \pm 0.31$ & $0.89 \pm 0.17$ & $1.39 \pm 0.17$ & $1.44 \pm 0.18$ & 1.5 & K4 (K4..M1) & $1.62(0.17 . .1 .62)$ \\
\hline J3414506+2537534 & $19.50 \pm 0.29$ & $18.07 \pm 0.18$ & $1.08 \pm 0.34$ & $1.42 \pm 0.21$ & $2.17 \pm 0.20$ & $2.29 \pm 0.24$ & 4.3 & M3 (M2..M4) & $1.31(0.11 . .1 .47)$ \\
\hline $\mathrm{J} 3414516+2525441$ & $18.78 \pm 0.27$ & $17.83 \pm 0.18$ & $0.59 \pm 0.32$ & $1.13 \pm 0.21$ & $1.66 \pm 0.23$ & $2.19 \pm 0.22$ & 5.9 & M0 (M0..M3) & $1.33(0.29 . .1 .33)$ \\
\hline $\mathrm{J} 3414517+2546110$ & $14.78 \pm 0.26$ & $14.28 \pm 0.17$ & $0.36 \pm 0.31$ & $0.74 \pm 0.17$ & $1.02 \pm 0.17$ & $1.05 \pm 0.17$ & 2.4 & K0 (G5..K2) & $0.52(0.26 . .1 .35)$ \\
\hline $\mathrm{J} 3414528+2548553$ & $18.70 \pm 0.27$ & $17.97 \pm 0.18$ & $0.27 \pm 0.32$ & $0.93 \pm 0.23$ & $1.55 \pm 0.24$ & $1.72 \pm$ & 2.5 & K4 (K4..M0) & $1.71(0.61 . .1 .71)$ \\
\hline $\mathrm{J} 3414532+2528049$ & $17.69 \pm 0.26$ & $16.44 \pm 0.17$ & $1.03 \pm 0.31$ & $1.01 \pm$ & $1.72 \pm 0.18$ & $1.91 \pm 0.18$ & 1.0 & & \\
\hline J3414554+2530331 & $16.73 \pm 0.26$ & $16.05 \pm 0.17$ & $0.38 \pm 0.31$ & $0.98 \pm 0.17$ & $1.45 \pm 0.18$ & $1.46 \pm 0.18$ & 2.8 & K4 (K3..M0) & $1.16(0.09 . .1 .45)$ \\
\hline $\mathrm{J} 3414561+2526168$ & $16.76 \pm 0.26$ & $15.66 \pm 0.17$ & $0.99 \pm 0.31$ & $1.08 \pm 0.17$ & $1.74 \pm 0.17$ & $1.89 \pm 0.17$ & 1.2 & & $0.40(0.22 . .1 .49)$ \\
\hline $\mathrm{J} 3414562+2541137$ & $15.92 \pm 0.26$ & $15.36 \pm 0.17$ & $0.32 \pm 0.31$ & $0.23 \pm 0.17$ & $0.52 \pm 0.18$ & $0.69 \pm 0.18$ & 1.6 & F6 (F4..G9) & $0.90(0.14 . .1 .25)$ \\
\hline $\mathrm{J} 3414570+2527447$ & $13.28 \pm 0.26$ & $12.73 \pm 0.17$ & $0.33 \pm 0.31$ & $0.68 \pm 0.17$ & $0.84 \pm 0.17$ & $0.89 \pm 0.17$ & 3.0 & G7 (F6..K1) & $0.82(0.64 . .1 .44)$ \\
\hline $\mathrm{J} 3414627+2541472$ & $19.01 \pm 0.27$ & $17.98 \pm 0.18$ & $0.76 \pm 0.33$ & $1.10 \pm 0.23$ & $-0.03 \pm$. & $1.98 \pm$ & 1.4 & M0 (K6..M2) & $1.00(0$ \\
\hline J3414641+2529177 & $17.51 \pm 0.26$ & $16.83 \pm 0.17$ & $0.49 \pm 0.31$ & $0.85 \pm 0.19$ & \pm 0.20 & 0.22 & 1.3 & & (.1.43) \\
\hline $\mathrm{J} 3414643+2544438$ & $17.58 \pm 0.26$ & $17.25 \pm 0.17$ & $0.11 \pm 0.31$ & $1.03 \pm 0.19$ & $1.57 \pm 0.20$ & $1.72 \pm 0.23$ & 7.3 & K4 (K3..K7) & $0.87(0.34 . .1 .36)$ \\
\hline $\mathrm{J} 3414653+2519365$ & $17.39 \pm 0.26$ & $16.96 \pm 0.17$ & $0.37 \pm 0.31$ & $0.67 \pm 0.20$ & $0.97 \pm 0.24$ & & 1.4 & & $2.1 .18)$ \\
\hline $\mathrm{J} 3414663+2552263$ & $17.29 \pm 0.26$ & $16.74 \pm 0.17$ & $0.38 \pm 0.31$ & $0.76 \pm 0.18$ & $0.99 \pm 0.21$ & \pm 0.25 & 2.8 & K0 & 43) \\
\hline J3414676+2534187 & $20.44 \pm 0.38$ & $18.71 \pm 0.19$ & $1.61 \pm 0.42$ & $2.73 \pm 0.21$ & $3.45 \pm 0.21$ & & 14.2 & M6.5 (M6..M7.5) & $38)$ \\
\hline $\mathrm{J} 3414680+2542327$ & $16.05 \pm 0.26$ & $15.47 \pm$ & 31 & 17 & 18 & & 5.0 & & 45) \\
\hline J3414685+2534548 & $17.06 \pm 0.26$ & $16.49 \pm 0.17$ & $0.37 \pm 0.31$ & $1.05 \pm$ & & & 4. & $\mathrm{~K} 4$ & \\
\hline J3414693+2541091 & $11.86 \pm 0.26$ & 11.43 & $0.43 \pm 0.31$ & 0.95 & $1.31=$ & 1.40 & 2.9 & $\mathrm{~K} 4$ & 34) \\
\hline J3414705+2545306 & $19.56 \pm 0.29$ & 18.1 & $1.13 \pm 0.34$ & & & & 2.0 & & \\
\hline $\mathrm{J} 3414715+2549450$ & 16.11 & 15.5 & $0.33 \pm 0.31$ & 0.58 & 0.8 & 0.94 & 1.5 & G7 & \\
\hline $\mathrm{J} 3414727+2531238$ & $15.76 \pm 0.26$ & 15.24 & $0.51 \pm 0.31$ & $1.00 \pm$ & & $1.38=$ & 3.6 & K4 ( & 0.02 \\
\hline J3414767+2547589 & 18.25 & 16.9 & $1.26 \pm$ & 1.1 & 1.8 & & 2.5 & & \\
\hline $\mathrm{J} 3414791+2526324$ & $18.63 \pm 0.27$ & $18.03 \pm 0.18$ & $0.24 \pm 0.32$ & $1.46 \pm 0.21$ & $2.19 \pm 0.21$ & 2.01 & 18.9 & M0 & 1.3 \\
\hline $\mathrm{J} 3414805+2522054$ & $15.95 \pm 0.26$ & $14.98 \pm 0.17$ & $0.58 \pm 0.31$ & $0.61 \pm 0.17$ & $0.99 \pm 0.17$ & 1.04 & 2.3 & $\mathrm{~K} 1$ & 1.46 \\
\hline $\mathrm{J} 3414809+2552471$ & $16.79 \pm 0.26$ & $16.00 \pm 0.17$ & $0.51 \pm 0.31$ & $0.83 \pm 0.18$ & $1.18 \pm 0.18$ & 1.3 & 2.0 & K3 & 1.07 \\
\hline $\mathrm{J} 3414825+2550060$ & $17.30 \pm 0.26$ & 16.7 & $0.48 \pm 0.31$ & $0.80 \pm 0.19$ & 0.21 & & 1.4 & $\mathrm{~K} 3$ & 49) \\
\hline $\mathrm{J} 3414830+2541475$ & $21.00 \pm 0.58$ & $19.15 \pm 0.21$ & $1.50 \pm 0.62$ & $1.94 \pm 0.28$ & $2.69 \pm 0.28$ & 0.36 & 3.5 & M5 (M5..M6.5) & $.1 .32)$ \\
\hline $\mathrm{J} 3414835+2522424$ & $17.05 \pm 0.26$ & $16.05 \pm 0.17$ & $0.87 \pm 0.31$ & \pm 0.17 & $1.69 \pm 0.18$ & 0.18 & 1.4 & 6..M2) & .43) \\
\hline J3414836+2547310 & $18.85 \pm 0.27$ & $18.04 \pm 0.17$ & $0.63 \pm 0.32$ & $1.29 \pm 0.23$ & $1.74 \pm 0.26$ & $1.57 \pm$ & 3.8 & M0 (K4..M2) & $0.67(0$. \\
\hline $\mathrm{J} 3414840+2534114$ & $15.95 \pm 0.26$ & $15.45 \pm 0.17$ & $0.44 \pm 0.31$ & $1.04 \pm 0.17$ & $1.31 \pm 0.18$ & 8 & 3.8 & K4 (G7..K6) & 33) \\
\hline J3414849+2551365 & $15.04 \pm 0.26$ & $14.34 \pm 0.17$ & $0.50 \pm 0.31$ & $0.89 \pm 0.17$ & $1.32 \pm 0.17$ & $1.47 \pm 0.17$ & 1. & K4 (K2..K6) & $0.77(0.39 . .1 .38)$ \\
\hline J3414875+2529586 & $17.65 \pm 0.26$ & & $0.99 \pm 0.31$ & & & & 1. & & \\
\hline J3414887+2538185 & $17.61 \pm 0.26$ & $17.28 \pm 0.17$ & $0.33 \pm 0.31$ & $0.96 \pm 0.20$ & $1.33 \pm 0.24$ & $1.65 \pm 0.27$ & 2. & K4 (K0..K4) & $0.00(0.00 . .0 .92)$ \\
\hline J3414898+2533379 & $17.33 \pm 0.26$ & & $0.32 \pm 0.31$ & & & & 7. & & \\
\hline+2530576 & 17.91 & 17.27 & $3 \pm 0.32$ & .20 & .24 & 1.00 & 4. & K0 & 1.17( \\
\hline J34149 & & & & & & & 3. & & \\
\hline $\mathrm{J} 3414950+2553070$ & $18.62 \pm 0.27$ & $17.37 \pm 0.17$ & $0.94 \pm 0.32$ & $0.57 \pm 0.23$ & $0.90 \pm 0.28$ & $1.77 \pm 0.22$ & 9.7 & K7 (K3..M1) & $1.19(0.34 . .1 .48)$ \\
\hline $\mathrm{J} 3414969+2534513$ & & & $2 \pm 0.31$ & & 1.2 & 1. & 2 & & \\
\hline J3414976+2541376 & $19.67 \pm 0.31$ & 18.3 & $1.16 \pm 0.36$ & $1.47 \pm 0.23$ & $2.18 \pm$ & $3.00 \pm$ & 1.9 & M3 (M1...M4) & $0.69(0.05 . .1 .45)$ \\
\hline $\mathrm{J} 3415000+2543361$ & $18.26 \pm 0.27$ & 17. & $0.45 \pm 0.32$ & $=0.21$ & \pm 0.23 & 3 & 2.8 & K4 (K3..M0) & $1.29(0.33 . .1 .42)$ \\
\hline+2538420 & $14.33 \pm 0.26$ & 13. & $0.39 \pm 0.31$ & 0.17 & 0.17 & 17 & 4.2 & K3 & $0.00(0.00 . .1 .46)$ \\
\hline $\mathrm{J} 3415019+2527199$ & $18.54 \pm 0.27$ & & \pm 0 & 1. & & & 22.2 & $\mathrm{M}$ & ...1.47) \\
\hline $\mathrm{J} 3415027+2532597$ & $17.73 \pm 0.26$ & $16.95 \pm 0.17$ & $0.34 \pm 0.31$ & $0.93 \pm 0.18$ & $1.50 \pm 0.18$ & $1.50 \pm 0.20$ & 2.5 & K4 (K4..M0) & $1.65(0.36 . .1 .70)$ \\
\hline $\mathrm{J} 3415038+2531040$ & $17.69 \pm 0.26$ & & $0.67 \pm 0.31$ & & 8 & & 6.0 & MOQ & $1.90(0.18 . .1 .90)$ \\
\hline $\mathrm{J} 3415057+2530077$ & $17.75 \pm 0.26$ & $17.42 \pm 0.17$ & $0.28 \pm 0.31$ & $1.22 \pm 0.19$ & $0.93 \pm 0.33$ & $1.70 \pm 0.27$ & 12.4 & K4 (F6..K7) & $0.22(0.22 . .1 .46)$ \\
\hline J3415086+2532399 & $19.59 \pm 0.29$ & $18.26 \pm 0.18$ & $1.23 \pm 0.34$ & $1.46 \pm 0.23$ & $1.97 \pm 0.27$ & 273 & 1.7 & M3 (M0..M3) & $0.39(0.17 . .1 .46)$ \\
\hline+2542203 & $3 \pm 0.26$ & 0.17 & $5 \pm 0.31$ & 17 & $1.41=$ & & 4.1 & $\mathrm{~K} 4$ & .34) \\
\hline+2536245 & $18.20 \pm 0.26$ & 17.20 & $0.67 \pm$ & & & $1.90=$ & 3.8 & & $7.1 .26)$ \\
\hline+2523013 & $15.39 \pm 0.26$ & 14.7 & $0.43 \pm 0.31$ & -0.42 & & 0.44 & 15.3 & & $0.1 .32)$ \\
\hline $\mathrm{J} 341$ & & & & & & & 4. & & ..1.34) \\
\hline J34151 & & & 0. & & & & 3. & & \\
\hline+2522545 & & & $0.37 \pm 0.31$ & & & $0.17 \pm 0.18$ & 2. & F0 ( & 1.1.48) \\
\hline J34151 & & & & & & & 1.0 & & \\
\hline J3415207+2542096 & & 18 & 0 & 1 & & 0.25 & 14.3 & 13) & 9.1.47) \\
\hline $\mathrm{J} 34152$ & 16. & & 1 & & & & 1.9 & & 50) \\
\hline J34152 & & & 1 & & & & 3. & & $.1 .45)$ \\
\hline $\mathrm{J} 34152$ & & & & & & & 2. & & \\
\hline J34152C & $8 \pm 0.26$ & $0+017$ & $0.47 \pm 0.31$ & \pm 0.17 & .17 & 1. & 1.8 & K3 & $0.79(0.03 . .1 .23)$ \\
\hline & & & & & & & 1. & & \\
\hline $\mathrm{J} 3415307+2534138$ & $15.70 \pm 0.26$ & $15.15 \pm 0.17$ & $0.51 \pm 0.31$ & $0.99 \pm 0.17$ & $1.28 \pm 0.18$ & $1.43 \pm 0.18$ & 3.3 & K4 (G9..K6) & $0.16(0.08 . .1 .16)$ \\
\hline J3415318+2540424 & $17.51 \pm 0.26$ & & & & & & 1.9 & K4 (K3...M0) & $1.21(0.16 . .1 .44)$ \\
\hline+2548541 & & $16.06 \pm 0.17$ & $0.55 \pm 0.31$ & $7 \pm 0.18$ & 1. & 21 & 2. & 7..K3) & 45) \\
\hline 2536418 & & & & & & & 4. & & \\
\hline $\mathrm{J} 3415333+2552276$ & $14.78 \pm 0.26$ & 14.0 & $0.51 \pm 0.31$ & $0.88 \pm 0$ & 1.3 & $1.42 \pm$ & 1.5 & K4 & 0.96 \\
\hline & & & & & & & 1.7 & & \\
\hline $\mathrm{J} 3415347+2521114$ & $17.11 \pm 0.26$ & $16.41 \pm 0.17$ & $0.40 \pm 0.31$ & $0.30 \pm 0.19$ & $0.71 \pm 0.20$ & $0.60 \pm 0.24$ & 1.5 & G0 (F7..G5) & $38)$ \\
\hline+2532288 & $16.10 \pm 0$ & & & & & & 2.9 & K4 (K4..M0) & $1.42(0.25 . .1 .48)$ \\
\hline $\mathrm{J} 3415368+2540581$ & $16.97 \pm 0.26$ & $15.88 \pm 0.17$ & $0.73 \pm 0.31$ & $-0.47 \pm 0.21$ & $-0.03 \pm 0.22$ & $0.18 \pm 0.23$ & 16.2 & F0 (F0..G4) & $1.34(0.08 . .1 .34)$ \\
\hline & & & $0.44 \pm 0$ & & & $3.41 \pm 0.30$ & 62. & M5 (M2..M7.5) & $0.54(0.03 . .1 .16)$ \\
\hline $\mathrm{J} 3415399+2544552$ & $19.70 \pm 0.30$ & $18.34 \pm 0.18$ & $1.04 \pm 0.35$ & $8 \pm 0.22$ & $2.08 \pm 0.24$ & 2.24 & 4.2 & M3 (M2..M4) & $1.19(0.06 . .1 .45)$ \\
\hline $\mathrm{J} 3415462+2526427$ & $13.24 \pm 0.26$ & $12.63 \pm 0.17$ & $0.50 \pm 0.31$ & $0.89 \pm 0.17$ & $1.22 \pm 0.17$ & $1.25 \pm 0.17$ & 3.0 & K3 (G6..K6) & $0.42(0.11 . .1 .45)$ \\
\hline
\end{tabular}




\begin{tabular}{|c|c|c|c|c|c|c|c|c|c|}
\hline \multirow[b]{2}{*}{ 2MASS-ID } & \multicolumn{2}{|c|}{ measured magnitudes } & & extinction c & ected colors & & & & \\
\hline & Rmag & Imag & R-I & I-J & $\mathrm{I}-\mathrm{H}$ & $\mathrm{I}-\mathrm{K}_{s}$ & $\chi^{2}$ & SpT & $\mathrm{Av}$ \\
\hline $\mathrm{J} 3415466+2551592$ & $19.18 \pm 0.28$ & $17.86 \pm 0.18$ & $1.04 \pm 0.33$ & $1.48 \pm 0.20$ & $2.19 \pm 0.20$ & $2.29 \pm 0.23$ & 5.6 & M3 (M1..M4) & $1.05(0.37 . .1 .45)$ \\
\hline $\mathrm{J} 3415469+2550364$ & $17.90 \pm 0.26$ & $17.01 \pm 0.17$ & $0.74 \pm 0.31$ & $1.06 \pm 0.19$ & $1.81 \pm 0.19$ & $1.90 \pm 0.21$ & 2.1 & M0 (K5..M1) & $0.57(0.20 . .1 .48)$ \\
\hline $\mathrm{J} 3415471+2547385$ & $20.21 \pm 0.36$ & $19.12 \pm 0.22$ & $1.09 \pm 0.42$ & $2.43 \pm 0.27$ & $2.52 \pm 0.37$ & $2.80 \pm \ldots$ & 13.2 & M5 (M3..M6) & $0.00(0.00 . .1 .37)$ \\
\hline $\mathrm{J} 3415473+2543094$ & $17.83 \pm 0.26$ & $17.28 \pm 0.17$ & $0.45 \pm 0.31$ & $1.04 \pm 0.19$ & $1.25 \pm 0.23$ & $1.46 \pm 0.28$ & 3.8 & $\mathrm{~K} 4(\mathrm{~K} 0 . . \mathrm{K} 7)$ & $0.37(0.06 . .1 .50)$ \\
\hline $\mathrm{J} 3415497+2543570$ & $18.33 \pm 0.27$ & $17.13 \pm 0.17$ & $1.09 \pm 0.32$ & $0.44 \pm 0.22$ & $1.16 \pm 0.23$ & $1.52 \pm 0.25$ & 6.5 & K7 (G7..M0) & $0.38(0.14 . .1 .42)$ \\
\hline $\mathrm{J} 3415526+2533121$ & $15.77 \pm 0.26$ & $15.24 \pm 0.17$ & $0.44 \pm 0.31$ & $1.01 \pm 0.17$ & $1.34 \pm 0.18$ & $1.45 \pm 0.18$ & 3.2 & K4 (G9..K4) & $0.31(0.14 . .1 .41)$ \\
\hline $\mathrm{J} 3415550+2539468$ & $19.37 \pm 0.28$ & $19.13 \pm 0.22$ & $0.02 \pm 0.36$ & $2.45 \pm 0.25$ & $2.76 \pm 0.30$ & $2.86 \pm \ldots$ & 46.0 & M3 (K2..M6) & $0.85(0.01 . .1 .47)$ \\
\hline $\mathrm{J} 3415575+2548443$ & $18.60 \pm 0.27$ & $17.71 \pm 0.17$ & $0.44 \pm 0.32$ & $0.79 \pm 0.22$ & $1.45 \pm 0.22$ & $1.08 \pm$ & 1.2 & $\mathrm{~K} 4$ (K4..K7) & $1.70(1.16 . .1 .70)$ \\
\hline $\mathrm{J} 3415582+2540498$ & $19.37 \pm 0.28$ & $17.73 \pm 0.18$ & $1.53 \pm 0.33$ & $0.78 \pm 0.24$ & $1.58 \pm 0.25$ & $1.77 \pm 0.30$ & 5.5 & M2 (K5..M3) & $0.41(0.10 . .1 .48)$ \\
\hline $\mathrm{J} 3415608+2550343$ & $16.96 \pm 0.26$ & $15.88 \pm 0.17$ & $0.78 \pm 0.31$ & $1.12 \pm 0.17$ & $1.70 \pm 0.17$ & $1.89 \pm 0.17$ & 1.9 & M0 (M0..M2) & $1.12(0.44 . .1 .34)$ \\
\hline $\mathrm{J} 3415630+2519340$ & $17.94 \pm 0.27$ & $17.74 \pm 0.18$ & $0.20 \pm 0.32$ & $1.09 \pm 0.23$ & $1.47 \pm 0.28$ & $1.80 \pm \ldots$ & 4.1 & K4 (G7..K4) & $0.00(0.00 . .0 .95)$ \\
\hline $\mathrm{J} 3415631+2545392$ & $12.57 \pm 0.26$ & $12.10 \pm 0.17$ & $0.44 \pm 0.31$ & $0.89 \pm 0.17$ & $1.24 \pm 0.17$ & $1.31 \pm 0.17$ & 2.6 & K3 (G4..K4) & $0.09(0.01 . .1 .29)$ \\
\hline $\mathrm{J} 3415632+2530014$ & $14.56 \pm 0.26$ & $13.86 \pm 0.17$ & $0.33 \pm 0.31$ & $1.05 \pm 0.17$ & $1.43 \pm 0.17$ & $1.49 \pm 0.17$ & 4.1 & K4 (K4..M1) & $1.39(0.13 . .1 .42)$ \\
\hline $\mathrm{J} 3415640+2540326$ & $14.18 \pm 0.26$ & $13.73 \pm 0.17$ & $0.41 \pm 0.31$ & $0.96 \pm 0.17$ & $1.26 \pm 0.17$ & $1.29 \pm 0.17$ & 4.0 & $\mathrm{~K} 3(\mathrm{G} 3 . . \mathrm{K} 3)$ & $0.13(0.13 . .1 .48)$ \\
\hline $\mathrm{J} 3415651+2549576$ & $15.13 \pm 0.26$ & $14.42 \pm 0.17$ & $0.51 \pm 0.31$ & $0.91 \pm 0.17$ & $1.34 \pm 0.17$ & $1.44 \pm 0.17$ & 1.7 & $\mathrm{~K} 4$ (K1..K7) & $0.72(0.42 . .1 .40)$ \\
\hline $\mathrm{J} 3415664+2538081$ & $17.68 \pm 0.26$ & $17.07 \pm 0.17$ & $0.46 \pm 0.31$ & $0.91 \pm 0.19$ & $1.39 \pm 0.20$ & $1.43 \pm 0.24$ & 1.5 & K4 (K0..K5) & $0.56(0.22 . .1 .45)$ \\
\hline $\mathrm{J} 3415666+2527149$ & $18.90 \pm 0.27$ & $18.58 \pm 0.21$ & $-0.18 \pm 0.34$ & $2.06 \pm 0.22$ & $2.24 \pm 0.25$ & $2.29 \pm 0.29$ & 46.7 & M0 (K3..M5) & $1.89(0.04 . .1 .89)$ \\
\hline $\mathrm{J} 3415695+2547471$ & $17.87 \pm 0.26$ & $17.26 \pm 0.17$ & $0.36 \pm 0.31$ & $0.42 \pm 0.24$ & $0.16 \pm \ldots$ & $1.46 \pm \ldots$ & 1.0 & G6 (F7..K1) & $0.95(0.00 . .1 .39)$ \\
\hline $\mathrm{J} 3415699+2533180$ & $15.40 \pm 0.26$ & $15.18 \pm 0.17$ & $0.15 \pm 0.31$ & $-1.12 \pm 0.19$ & $-0.62 \pm 0.22$ & $-0.49 \pm 0.26$ & 19.5 & $\mathrm{~B} 0$ (B0..B7) & $0.26(0.04 . .0 .97)$ \\
\hline $\mathrm{J} 3415722+2520331$ & $18.54 \pm 0.27$ & $17.06 \pm 0.17$ & $1.25 \pm 0.32$ & $1.41 \pm 0.18$ & $1.97 \pm 0.19$ & $2.23 \pm 0.19$ & 1.7 & M3 (M2..M4) & $0.85(0.02 . .1 .31)$ \\
\hline $\mathrm{J} 3415729+2546512$ & $19.23 \pm 0.28$ & $18.38 \pm 0.18$ & $0.57 \pm 0.33$ & $1.86 \pm 0.21$ & $2.48 \pm 0.22$ & $2.47 \pm 0.27$ & 24.8 & M3 (M0..M5) & $1.06(0.06 . .1 .08)$ \\
\hline $\mathrm{J} 3415748+2524249$ & $16.37 \pm 0.26$ & $15.36 \pm 0.17$ & $0.81 \pm 0.31$ & $1.14 \pm 0.17$ & $1.70 \pm 0.17$ & $1.84 \pm 0.17$ & 2.1 & M0 (K7..M2) & $0.77(0.09 . .1 .34)$ \\
\hline $\mathrm{J} 3415768+2542012$ & $18.41 \pm 0.27$ & $18.20 \pm 0.18$ & $-0.07 \pm 0.32$ & $1.30 \pm 0.23$ & $1.60 \pm 0.28$ & $1.89 \pm 0.32$ & 12.4 & $\mathrm{~K} 4$ (K1..K7) & $1.07(0.03 . .1 .39)$ \\
\hline $\mathrm{J} 3415803+2551445$ & $16.29 \pm 0.26$ & $15.45 \pm 0.17$ & $0.52 \pm 0.31$ & $0.79 \pm 0.17$ & $1.18 \pm 0.18$ & $1.32 \pm 0.18$ & 1.6 & $\mathrm{~K} 3$ (K2..M0) & $1.18(0.03 . .1 .38)$ \\
\hline $\mathrm{J} 3415809+2529309$ & $17.92 \pm 0.26$ & $17.66 \pm 0.17$ & $0.12 \pm 0.32$ & $1.07 \pm 0.21$ & $1.38 \pm 0.27$ & $1.89 \pm 0.27$ & 8.0 & K4 (K0..K6) & $0.51(0.18 . .1 .43)$ \\
\hline $\mathrm{J} 3415827+2541337$ & $14.39 \pm 0.26$ & $13.72 \pm 0.17$ & $0.48 \pm 0.31$ & $-1.31 \pm 0.17$ & $-0.80 \pm 0.18$ & $-0.70 \pm 0.18$ & 40.0 & $\mathrm{~B} 0(\mathrm{~B} 0 . . \mathrm{A} 0)$ & $0.72(0.02 . .0 .93)$ \\
\hline $\mathrm{J} 3415838+2525502$ & $16.94 \pm 0.26$ & $16.01 \pm 0.17$ & $0.71 \pm 0.31$ & $1.15 \pm 0.17$ & $1.75 \pm 0.18$ & $1.91 \pm 0.18$ & 2.8 & M0 (K7..M2) & $0.79(0.09 . .1 .38)$ \\
\hline $\mathrm{J} 3415841+2548094$ & $19.19 \pm 0.28$ & $18.57 \pm 0.19$ & $0.12 \pm 0.34$ & $1.55 \pm 0.23$ & $2.15 \pm 0.25$ & $2.28 \pm 0.28$ & 20.2 & M0 (K4..M3) & $1.86(0.64 . .1 .86)$ \\
\hline $\mathrm{J} 3415851+2547302$ & $17.49 \pm 0.26$ & $16.85 \pm 0.17$ & $0.40 \pm 0.31$ & $0.96 \pm 0.18$ & $1.41 \pm 0.20$ & $1.48 \pm 0.21$ & 2.2 & K4 (K3..K6) & $0.92(0.67 . .1 .34)$ \\
\hline $\mathrm{J} 3415864+2533546$ & $13.38 \pm 0.26$ & $12.88 \pm 0.17$ & $0.43 \pm 0.31$ & $1.05 \pm 0.17$ & $1.34 \pm 0.17$ & $1.45 \pm 0.17$ & 4.0 & $\mathrm{~K} 4(\mathrm{~K} 0 . . \mathrm{K} 7)$ & $0.25(0.04 . .1 .04)$ \\
\hline $\mathrm{J} 3415890+2527148$ & $16.96 \pm 0.26$ & $16.21 \pm 0.17$ & $0.37 \pm 0.31$ & $0.71 \pm 0.18$ & $1.03 \pm 0.18$ & $1.04 \pm 0.19$ & 2.0 & K0 (G9..K5) & $1.45(0.18 . .1 .50)$ \\
\hline $\mathrm{J} 3415893+2541241$ & $12.95 \pm 0.26$ & $12.54 \pm 0.17$ & $0.39 \pm 0.31$ & $0.96 \pm 0.17$ & $1.27 \pm 0.17$ & $1.30 \pm 0.17$ & 4.1 & K3 (G6..K4) & $0.11(0.07 . .1 .20)$ \\
\hline $\mathrm{J} 3415903+2522361$ & $18.41 \pm 0.27$ & $17.98 \pm 0.18$ & $-0.00 \pm 0.32$ & $0.90 \pm 0.24$ & $1.85 \pm 0.22$ & $1.72 \pm \ldots$ & 10.1 & $\mathrm{~K} 4$ (K2..M1) & $1.63(0.66 . .1 .63)$ \\
\hline $\mathrm{J} 3415914+2553375$ & $16.19 \pm 0.26$ & $15.46 \pm 0.17$ & $0.40 \pm 0.31$ & $0.73 \pm 0.17$ & $0.97 \pm 0.18$ & $1.05 \pm 0.18$ & 2.5 & K0 (G8..K6) & $1.23(0.16 . .1 .42)$ \\
\hline $\mathrm{J} 3415956+2526104$ & $15.07 \pm 0.26$ & $14.29 \pm 0.17$ & $0.45 \pm 0.31$ & $0.94 \pm 0.17$ & $1.40 \pm 0.17$ & $1.45 \pm 0.17$ & 1.9 & K4 (K3..M0) & $1.24(0.32 . .1 .45)$ \\
\hline $\mathrm{J} 3420001+2529515$ & $20.24 \pm 0.34$ & $21.19 \pm 1.05$ & $-1.42 \pm 1.10$ & $4.11 \pm 1.06$ & $4.47 \pm 1.07$ & $4.82 \pm 1.07$ & 26.6 & M3 (K7..M6) & $1.75(0.04 . .1 .77)$ \\
\hline $\mathrm{J} 3420022+2539201$ & $18.41 \pm 0.27$ & $17.58 \pm 0.17$ & $0.39 \pm 0.32$ & $0.81 \pm 0.21$ & $1.04 \pm 0.25$ & $1.86 \pm 0.22$ & 8.9 & $\mathrm{~K} 4$ (K2..M2) & $1.66(0.11 . .1 .66)$ \\
\hline $\mathrm{J} 3420024+2536063$ & $19.07 \pm 0.27$ & $18.35 \pm 0.18$ & $0.25 \pm 0.33$ & $1.17 \pm 0.24$ & $2.07 \pm 0.22$ & $2.36 \pm 0.25$ & 15.6 & M0 (K6..M2) & $1.79(0.64 . .1 .79)$ \\
\hline $\mathrm{J} 3420041+2524430$ & $13.31 \pm 0.26$ & $12.70 \pm 0.17$ & $0.24 \pm 0.31$ & $0.54 \pm 0.17$ & $0.66 \pm 0.17$ & $0.67 \pm 0.17$ & 2.6 & F7 (F7..G8) & $1.40(0.61 . .1 .48)$ \\
\hline $\mathrm{J} 3420043+2525591$ & $13.37 \pm 0.26$ & $12.81 \pm 0.17$ & $0.33 \pm 0.31$ & $0.67 \pm 0.17$ & $0.86 \pm 0.17$ & $0.88 \pm 0.17$ & 2.8 & G7 (F6..K3) & $0.85(0.22 . .1 .40)$ \\
\hline $\mathrm{J} 3420052+2528215$ & $12.87 \pm 0.26$ & $12.38 \pm 0.17$ & $0.39 \pm 0.31$ & $0.80 \pm 0.17$ & $0.97 \pm 0.17$ & $1.03 \pm 0.17$ & 3.9 & $\mathrm{~K} 0(\mathrm{~F} 7 . . \mathrm{K} 0)$ & $0.38(0.38 . .1 .44)$ \\
\hline $\mathrm{J} 3420086+2533009$ & $15.17 \pm 0.26$ & $14.56 \pm 0.17$ & $0.36 \pm 0.31$ & $1.06 \pm 0.17$ & $1.38 \pm 0.17$ & $1.50 \pm 0.17$ & 4.2 & $\mathrm{~K} 4$ (K2..K7) & $0.96(0.54 . .1 .44)$ \\
\hline $\mathrm{J} 3420095+2537540$ & $17.47 \pm 0.26$ & $16.95 \pm 0.17$ & $0.49 \pm 0.31$ & $1.11 \pm 0.18$ & $1.31 \pm 0.21$ & $1.22 \pm 0.28$ & 6.8 & $\mathrm{~K} 4(\mathrm{G} 0 . . \mathrm{K} 4)$ & $0.14(0.14 . .1 .46)$ \\
\hline $\mathrm{J} 3420102+2528470$ & $18.75 \pm 0.27$ & $17.26 \pm 0.17$ & $1.49 \pm 0.32$ & $1.28 \pm 0.19$ & $1.88 \pm 0.20$ & $1.83 \pm 0.24$ & 3.9 & M3 (M0..M3) & $0.00(0.00 . .1 .12)$ \\
\hline $\mathrm{J} 3420104+2540329$ & $16.47 \pm 0.26$ & $15.95 \pm 0.17$ & $0.50 \pm 0.31$ & $0.98 \pm 0.18$ & $1.38 \pm 0.18$ & $1.36 \pm 0.19$ & 3.0 & $\mathrm{~K} 4(\mathrm{~K} 0 . . \mathrm{K} 4)$ & $0.08(0.08 . .1 .16)$ \\
\hline $\mathrm{J} 3420110+2519381$ & $16.89 \pm 0.26$ & $16.33 \pm 0.17$ & $0.38 \pm 0.31$ & $0.48 \pm 0.18$ & $0.93 \pm 0.19$ & $0.86 \pm 0.23$ & 1.3 & G7 (F5..K2) & $0.65(0.13 . .1 .46)$ \\
\hline $\mathrm{J} 3420188+2525402$ & $16.62 \pm 0.26$ & $15.84 \pm 0.17$ & $0.24 \pm 0.31$ & $0.12 \pm 0.18$ & $0.21 \pm 0.19$ & $0.28 \pm 0.19$ & 1.1 & A9 (A9..F5) & $2.05(1.45 . .2 .05)$ \\
\hline $\mathrm{J} 3420208+2530210$ & $19.02 \pm 0.27$ & $17.38 \pm 0.17$ & $1.64 \pm 0.32$ & $1.49 \pm 0.19$ & $2.18 \pm 0.19$ & $2.34 \pm 0.21$ & 1.3 & M4 (M3..M4) & $0.00(0.00 . .0 .65)$ \\
\hline $\mathrm{J} 3420240+2551055$ & $17.11 \pm 0.26$ & $15.79 \pm 0.17$ & $1.20 \pm 0.31$ & $1.10 \pm 0.18$ & $1.74 \pm 0.18$ & $1.95 \pm 0.18$ & 1.1 & M2 (M1..M2) & $0.48(0.26 . .0 .84)$ \\
\hline $\mathrm{J} 3420242+2527095$ & $16.31 \pm 0.26$ & $15.65 \pm 0.17$ & $0.40 \pm 0.31$ & $0.45 \pm 0.17$ & $0.95 \pm 0.18$ & $0.93 \pm 0.19$ & 1.2 & G9 (F9..K3) & $0.99(0.02 . .1 .47)$ \\
\hline $\mathrm{J} 3420254+2535588$ & $16.44 \pm 0.26$ & $15.93 \pm 0.17$ & $0.34 \pm 0.31$ & $1.04 \pm 0.17$ & $1.37 \pm 0.17$ & $1.53 \pm 0.18$ & 3.9 & $\mathrm{~K} 4$ (K1..K6) & $0.62(0.22 . .1 .22)$ \\
\hline $\mathrm{J} 3420256+2549080$ & $19.29 \pm 0.28$ & $17.96 \pm 0.18$ & $1.20 \pm 0.33$ & $1.43 \pm 0.21$ & $1.88 \pm 0.24$ & $2.41 \pm 0.25$ & 3.1 & M3 (M0..M4) & $0.52(0.11 . .1 .47)$ \\
\hline $\mathrm{J} 3420276+2531285$ & $18.27 \pm 0.27$ & $17.88 \pm 0.17$ & $0.03 \pm 0.32$ & $1.11 \pm 0.21$ & $1.69 \pm 0.22$ & $1.72 \pm 0.28$ & 9.1 & $\mathrm{~K} 4$ (K2..M1) & $1.36(0.05 . .1 .50)$ \\
\hline $\mathrm{J} 3420283+2545582$ & $16.39 \pm 0.26$ & $15.82 \pm 0.17$ & $0.53 \pm 0.31$ & $0.89 \pm 0.18$ & $1.38 \pm 0.18$ & $1.38 \pm 0.18$ & 1.9 & $\mathrm{~K} 4$ (G7..K4) & $0.17(0.04 . .1 .30)$ \\
\hline $\mathrm{J} 3420291+2526483$ & $19.14 \pm 0.28$ & $18.02 \pm 0.18$ & $1.12 \pm 0.33$ & $1.46 \pm 0.22$ & $1.87 \pm 0.27$ & $2.27 \pm$ & 3.0 & M3 (K7..M3) & $0.00(0.00 . .1 .43)$ \\
\hline $\mathrm{J} 3420294+2549384$ & $17.18 \pm 0.26$ & $16.77 \pm 0.17$ & $0.41 \pm 0.31$ & $0.99 \pm 0.18$ & $1.34 \pm 0.21$ & $1.49 \pm 0.23$ & 2.6 & K4 (K0..K4) & $0.00(0.00 . .0 .88)$ \\
\hline $\mathrm{J} 3420305+2554233$ & $17.68 \pm 0.26$ & $16.40 \pm 0.17$ & $1.04 \pm 0.31$ & $2.63 \pm 0.17$ & $2.84 \pm 0.17$ & $2.88 \pm 0.17$ & 50.1 & M5 (M3..M7.5) & $0.91(0.03 . .1 .26)$ \\
\hline $\mathrm{J} 3420312+2547594$ & $17.31 \pm 0.26$ & $16.71 \pm 0.17$ & $0.50 \pm 0.31$ & $0.94 \pm 0.18$ & $1.41 \pm 0.19$ & $1.33 \pm 0.22$ & 2.7 & K4 (G9..K6) & $0.41(0.03 . .1 .50)$ \\
\hline $\mathrm{J} 3420322+2531093$ & $18.52 \pm 0.27$ & $18.19 \pm 0.18$ & $0.00 \pm 0.32$ & $1.24 \pm 0.24$ & $1.64 \pm \ldots$ & $2.00 \pm \ldots$ & 6.9 & K4 (G7..M2) & $1.23(0.11 . .1 .49)$ \\
\hline $\mathrm{J} 3420324+2544485$ & $17.46 \pm 0.26$ & $17.10 \pm 0.17$ & $0.31 \pm 0.31$ & $0.60 \pm 0.21$ & $1.11 \pm 0.24$ & $1.40 \pm$ & 1.2 & K0 (G6..K2) & $0.20(0.04 . .0 .73)$ \\
\hline $\mathrm{J} 3420326+2533022$ & $16.29 \pm 0.26$ & $15.70 \pm 0.17$ & $0.51 \pm 0.31$ & $0.97 \pm 0.17$ & $1.36 \pm 0.18$ & $1.38 \pm 0.18$ & 2.9 & K4 (G9..K7) & $0.29(0.03 . .1 .35)$ \\
\hline $\mathrm{J} 3420328+2550050$ & $17.70 \pm 0.26$ & $16.68 \pm 0.17$ & $0.70 \pm 0.31$ & $0.78 \pm 0.18$ & $1.37 \pm 0.19$ & $1.54 \pm 0.20$ & 1.1 & K6 (K4..M1) & $1.24(0.19 . .1 .50)$ \\
\hline $\mathrm{J} 3420330+2543072$ & $18.87 \pm 0.27$ & $18.88 \pm 0.19$ & $-0.41 \pm 0.33$ & $1.78 \pm 0.25$ & $2.66 \pm 0.24$ & $2.58 \pm$ & 46.1 & M0 (K0..M2) & $1.53(0.06 . .1 .55)$ \\
\hline $\mathrm{J} 3420341+2522393$ & $17.24 \pm 0.26$ & $15.13 \pm 0.17$ & $2.06 \pm 0.31$ & $1.74 \pm 0.17$ & $2.33 \pm 0.17$ & $2.61 \pm 0.17$ & 2.3 & M5 (M4..M5) & $0.21(0.11 . .1 .41)$ \\
\hline $\mathrm{J} 3420346+2553593$ & $17.15 \pm 0.26$ & $16.37 \pm 0.17$ & $0.49 \pm 0.31$ & $0.80 \pm 0.18$ & $1.25 \pm 0.18$ & $1.28 \pm 0.19$ & 1.5 & K3 (K3..K7) & $1.08(0.38 . .1 .20)$ \\
\hline $\mathrm{J} 3420360+2550212$ & $16.83 \pm 0.26$ & $15.80 \pm 0.17$ & $0.75 \pm 0.31$ & $1.10 \pm 0.17$ & $1.75 \pm 0.17$ & $1.90 \pm 0.17$ & 2.0 & M0 (M0..M1) & $1.04(0.67 . .1 .32)$ \\
\hline $\mathrm{J} 3420365+2534279$ & $17.28 \pm 0.26$ & $16.86 \pm 0.17$ & $0.30 \pm 0.31$ & $1.03 \pm 0.18$ & $1.61 \pm 0.19$ & $1.35 \pm 0.23$ & 5.9 & $\mathrm{~K} 4$ (K1..K7) & $0.46(0.21 . .0 .95)$ \\
\hline $\mathrm{J} 3420384+2528160$ & $17.78 \pm 0.26$ & $17.47 \pm 0.17$ & $-0.12 \pm 0.32$ & $1.27 \pm 0.19$ & $1.72 \pm 0.20$ & $1.73 \pm 0.22$ & 18.0 & K4 (K3..M1) & $1.61(0.61 . .1 .61)$ \\
\hline $\mathrm{J} 3420404+2550362$ & $17.17 \pm 0.26$ & $16.11 \pm 0.17$ & $0.78 \pm 0.31$ & $1.12 \pm 0.17$ & $1.71 \pm 0.18$ & $1.88 \pm 0.18$ & 2.0 & M0 (M0..M2) & $1.06(0.57 . .1 .25)$ \\
\hline $\mathrm{J} 3420413+2548079$ & $16.18 \pm 0.26$ & $15.51 \pm 0.17$ & $0.46 \pm 0.31$ & $0.91 \pm 0.17$ & $1.42 \pm 0.17$ & $1.42 \pm 0.18$ & 1.8 & $\mathrm{~K} 4$ (K2..K6) & $0.76(0.43 . .1 .29)$ \\
\hline $\mathrm{J} 3420425+2542283$ & $16.22 \pm 0.26$ & $15.61 \pm 0.17$ & $0.34 \pm 0.31$ & $1.01 \pm 0.17$ & $1.42 \pm 0.17$ & $1.52 \pm 0.18$ & 3.2 & K4 (K2..M0) & $1.02(0.01 . .1 .46)$ \\
\hline $\mathrm{J} 3420445+2545219$ & $19.97 \pm 0.34$ & $18.75 \pm 0.20$ & $0.73 \pm 0.39$ & $1.43 \pm 0.28$ & $2.24 \pm 0.25$ & $2.69 \pm 0.26$ & 11.9 & M3 (M3..M5) & $1.87(0.19 . .1 .87)$ \\
\hline $\mathrm{J} 3420450+2538289$ & $19.06 \pm 0.27$ & $17.75 \pm 0.17$ & $1.22 \pm 0.32$ & $1.58 \pm 0.20$ & $2.07 \pm 0.22$ & $2.01 \pm 0.27$ & 4.9 & M3 (M1..M3) & $0.33(0.07 . .1 .42)$ \\
\hline $\mathrm{J} 3420463+2543137$ & $17.86 \pm 0.26$ & $17.44 \pm 0.17$ & $0.27 \pm 0.31$ & $1.20 \pm 0.20$ & $1.27 \pm 0.25$ & $1.42 \pm$ & 7.4 & $\mathrm{~K} 4$ (G7..K7) & $0.56(0.28 . .1 .44)$ \\
\hline $\mathrm{J} 3420486+2539478$ & $15.00 \pm 0.26$ & $13.60 \pm 0.17$ & $1.07 \pm 0.31$ & $1.55 \pm 0.17$ & $2.11 \pm 0.17$ & $2.24 \pm 0.17$ & 6.1 & M3 (M3..M4) & $1.23(0.05 . .1 .46)$ \\
\hline $\mathrm{J} 3420491+2527166$ & $15.53 \pm 0.26$ & $14.91 \pm 0.17$ & $0.52 \pm 0.31$ & $0.95 \pm 0.17$ & $1.37 \pm 0.17$ & $1.37 \pm 0.17$ & 2.6 & $\mathrm{~K} 4$ (K0..K7) & $0.42(0.09 . .1 .49)$ \\
\hline $\mathrm{J} 3420508+2526323$ & $19.05 \pm 0.28$ & $17.58 \pm 0.17$ & $1.45 \pm 0.33$ & $0.85 \pm 0.23$ & $1.59 \pm \ldots$ & $1.83 \pm 0.29$ & 3.3 & M2 (K6..M2) & $0.11(0.11 . .1 .37)$ \\
\hline $\mathrm{J} 3420511+2545535$ & $14.29 \pm 0.26$ & $13.78 \pm 0.17$ & $0.47 \pm 0.31$ & $0.91 \pm 0.17$ & $1.23 \pm 0.17$ & $1.27 \pm 0.17$ & 3.1 & K3 (G7..K3) & $0.16(0.16 . .1 .23)$ \\
\hline $\mathrm{J} 3420525+2553594$ & $17.97 \pm 0.26$ & $17.36 \pm 0.17$ & $0.43 \pm 0.31$ & $0.74 \pm 0.21$ & $1.13 \pm 0.26$ & $1.81 \pm 0.23$ & 6.0 & K4 (G9..K6) & $0.66(0.23 . .1 .45)$ \\
\hline $\mathrm{J} 3420575+2550201$ & $18.16 \pm 0.27$ & $17.36 \pm 0.17$ & $0.50 \pm 0.32$ & $0.71 \pm 0.22$ & $0.96 \pm \ldots$ & $0.64 \pm \ldots$ & 1.0 & K3 (K0..K7) & $1.14(0.12 . .1 .45)$ \\
\hline $\mathrm{J} 3420577+2546282$ & $20.15 \pm 0.35$ & $18.32 \pm 0.18$ & $1.69 \pm 0.39$ & $1.94 \pm 0.21$ & $2.62 \pm 0.22$ & $2.65 \pm 0.26$ & 3.5 & M5 (M4..M5) & $0.54(0.03 . .1 .41)$ \\
\hline $\mathrm{J} 3420610+2531431$ & $17.73 \pm 0.26$ & $17.38 \pm 0.17$ & $0.25 \pm 0.31$ & $1.12 \pm 0.20$ & $1.54 \pm 0.22$ & $1.43 \pm 0.29$ & 5.7 & K4 (G9..K5) & $0.38(0.01 . .1 .31)$ \\
\hline
\end{tabular}




\begin{tabular}{|c|c|c|c|c|c|c|c|c|c|}
\hline \multirow[b]{2}{*}{ 2MASS-ID } & \multicolumn{2}{|c|}{ measured magnitudes } & & extinction $\mathrm{cc}$ & ected colors & & & & \\
\hline & Rmag & Imag & R-I & I-J & $\mathrm{I}-\mathrm{H}$ & $\mathrm{I}-\mathrm{K}_{s}$ & $\chi^{2}$ & SpT & $\mathrm{Av}$ \\
\hline J3420611+2551097 & $17.85 \pm 0.26$ & $16.94 \pm 0.17$ & $0.77 \pm 0.31$ & $0.72 \pm 0.19$ & $1.49 \pm 0.20$ & $1.53 \pm 0.22$ & 1.2 & K7 (K2..K7) & $0.56(0.31 . .1 .45)$ \\
\hline $\mathrm{J} 3420627+2535512$ & $15.58 \pm 0.26$ & $15.07 \pm 0.17$ & $0.42 \pm 0.31$ & $0.99 \pm 0.17$ & $1.40 \pm 0.17$ & $1.44 \pm 0.17$ & 2.7 & K4 (K0..K5) & $0.34(0.15 . .1 .04)$ \\
\hline $\mathrm{J} 3420630+2536396$ & $20.13 \pm 0.34$ & $20.12 \pm 0.42$ & $-0.03 \pm 0.53$ & $3.63 \pm 0.43$ & $3.81 \pm 0.48$ & $4.68 \pm \ldots$ & 42.7 & M5 (K7..M7) & $0.15(0.02 . .1 .24)$ \\
\hline $\mathrm{J} 3420636+2535404$ & $16.22 \pm 0.26$ & $15.99 \pm 0.17$ & $0.23 \pm 0.31$ & $-0.08 \pm 0.19$ & $0.60 \pm 0.20$ & $0.39 \pm 0.27$ & 6.2 & F3 (B9..F3) & $0.00(0.00 . .0 .94)$ \\
\hline $\mathrm{J} 3420646+2523167$ & $15.61 \pm 0.26$ & $14.91 \pm 0.17$ & $0.35 \pm 0.31$ & $0.59 \pm 0.17$ & $0.90 \pm 0.17$ & $0.87 \pm 0.17$ & 1.7 & G7 (G6..K4) & $1.34(0.19 . .1 .43)$ \\
\hline J3420652+2532069 & $19.07 \pm 0.27$ & $18.10 \pm 0.18$ & $0.66 \pm 0.33$ & $1.70 \pm 0.20$ & $2.28 \pm 0.21$ & $2.61 \pm 0.22$ & 19.5 & M3 (M2..M5) & $1.16(0.07 . .1 .49)$ \\
\hline $\mathrm{J} 3420684+2545142$ & $17.94 \pm 0.26$ & $17.16 \pm 0.17$ & $0.50 \pm 0.32$ & $0.82 \pm 0.20$ & $1.24 \pm 0.23$ & $1.59 \pm 0.23$ & 2.0 & K4 (K2..M0) & $1.06(0.17 . .1 .43)$ \\
\hline $\mathrm{J} 3420697+2527317$ & $19.96 \pm 0.32$ & $18.92 \pm 0.21$ & $0.60 \pm 0.38$ & $1.85 \pm 0.26$ & $2.21 \pm 0.31$ & $2.74 \pm 0.29$ & 16.0 & M3 (M0..M5) & $1.65(0.24 . .1 .65)$ \\
\hline $\mathrm{J} 3420724+2519024$ & $17.59 \pm 0.26$ & $16.91 \pm 0.17$ & $0.50 \pm 0.31$ & $0.71 \pm 0.20$ & $1.10 \pm$ & $0.82 \pm$ & 1.0 & K3 (G5..K6) & $0.67(0.17 . .1 .45)$ \\
\hline $\mathrm{J} 3420726+2552545$ & $12.35 \pm 0.26$ & $11.77 \pm 0.17$ & $0.32 \pm 0.31$ & $0.61 \pm 0.17$ & $0.90 \pm 0.17$ & $0.89 \pm 0.17$ & 1.9 & G7 (G1..K3) & $0.96(0.21 . .1 .27)$ \\
\hline $\mathrm{J} 3420735+2522205$ & $17.85 \pm 0.26$ & $16.81 \pm 0.17$ & $1.04 \pm 0.31$ & $1.08 \pm 0.19$ & $1.55 \pm 0.19$ & $1.88 \pm 0.20$ & 2.1 & M1 (K4...M1) & $0.00(0.00 . .1 .28)$ \\
\hline J3420753+2548532 & $17.63 \pm 0.26$ & $16.89 \pm 0.17$ & $0.33 \pm 0.31$ & $0.54 \pm 0.19$ & $0.88 \pm 0.21$ & $0.95 \pm 0.23$ & 1.1 & G7 (G7..K5) & $1.51(0.22 . .1 .51)$ \\
\hline $\mathrm{J} 3420767+2544116$ & $17.45 \pm 0.26$ & $16.67 \pm 0.17$ & $0.39 \pm 0.31$ & $0.95 \pm 0.18$ & $1.39 \pm 0.19$ & $1.51 \pm 0.19$ & 2.1 & K4 (K4..M0) & $1.49(0.19 . .1 .49)$ \\
\hline $\mathrm{J} 3420773+2528472$ & $17.01 \pm 0.26$ & $16.33 \pm 0.17$ & $0.50 \pm 0.31$ & $0.94 \pm 0.18$ & $1.39 \pm 0.18$ & $1.37 \pm 0.19$ & 2.3 & K4 (K1..K7) & $0.68(0.42 . .1 .47)$ \\
\hline $\mathrm{J} 3420778+2553288$ & $16.85 \pm 0.26$ & $15.92 \pm 0.17$ & $0.93 \pm 0.31$ & $1.03 \pm 0.17$ & $1.65 \pm 0.18$ & $1.80 \pm 0.18$ & 1.3 & M0 (K3...M0) & $0.02(0.02 . .1 .39)$ \\
\hline $\mathrm{J} 3420788+2532395$ & $18.79 \pm 0.27$ & $17.47 \pm 0.17$ & $1.14 \pm 0.32$ & $1.12 \pm 0.21$ & $1.76 \pm 0.21$ & $2.00 \pm 0.22$ & 1.0 & & $0.67(0.42 . .1 .17)$ \\
\hline $\mathrm{J} 3420817+2525588$ & $18.37 \pm 0.27$ & $17.90 \pm 0.18$ & $0.24 \pm 0.32$ & $0.68 \pm 0.27$ & $1.40 \pm 0.29$ & $1.95 \pm 0.27$ & 7.0 & K4 (K0..M0) & $0.90(0.15 .1 .18)$ \\
\hline $\mathrm{J} 3420821+2533429$ & $17.50 \pm 0.26$ & $16.83 \pm 0.17$ & $0.32 \pm 0.31$ & $0.92 \pm 0.18$ & $1.43 \pm 0.19$ & $1.61 \pm 0.19$ & 2.6 & & $1.33(0.34 . .1 .46)$ \\
\hline $\mathrm{J} 3420856+2544155$ & $18.69 \pm 0.27$ & $17.72 \pm 0.17$ & $0.70 \pm 0.32$ & $1.32 \pm 0.20$ & $1.85 \pm 0.22$ & $1.65 \pm 0.28$ & 6.9 & M0 (K6..M3) & $1.02(0.11 . .1 .36)$ \\
\hline $\mathrm{J} 3420858+2520435$ & $16.02 \pm 0.26$ & $15.41 \pm 0.17$ & $0.38 \pm 0.31$ & $0.54 \pm 0.17$ & $0.87 \pm 0.18$ & $0.89 \pm 0.18$ & 1.2 & G7 (F7..K0) & $0.87(0.51 . .1 .25)$ \\
\hline $\mathrm{J} 3420865+2553490$ & $15.45 \pm 0.26$ & $14.74 \pm 0.17$ & $0.63 \pm 0.31$ & $-1.45 \pm 0.19$ & $-0.85 \pm 0.21$ & $-0.77 \pm 0.24$ & 46.1 & В0 (B0..B9) & $0.31(0.18 .1 .46)$ \\
\hline $\mathrm{J} 3420885+2545248$ & $13.45 \pm 0.26$ & $12.93 \pm 0.17$ & $0.49 \pm 0.31$ & $0.99 \pm 0.17$ & $1.33 \pm 0.17$ & $1.41 \pm 0.17$ & 3.0 & & 3..1.14) \\
\hline $\mathrm{J} 3420887+2535223$ & $20.12 \pm 0.34$ & $19.17 \pm 0.21$ & $0.49 \pm 0.40$ & $1.68 \pm 0.31$ & $2.74 \pm 0.28$ & $2.53 \pm 0.36$ & 19.5 & M3 (M0..M6) & $1.74(0.04 . .1 .74)$ \\
\hline $\mathrm{J} 3420888+2520527$ & $16.00 \pm 0.26$ & $15.32 \pm 0.17$ & $0.07 \pm 0.31$ & $-0.71 \pm 0.18$ & $-0.69 \pm 0.20$ & $-0.65 \pm 0.22$ & 5.0 & B0 (B0..B7) & $9 . .2 .30)$ \\
\hline J3420910+2553389 & $15.38 \pm 0.26$ & $14.73 \pm 0.17$ & $0.24 \pm 0.31$ & $0.41 \pm 0.17$ & $0.54 \pm 0.17$ & $0.50 \pm 0.18$ & 1.8 & F5 (F5..G9) & $.52)$ \\
\hline J3420916+2523507 & $20.05 \pm 0.32$ & $18.30 \pm 0.18$ & $1.52 \pm 0.37$ & $1.54 \pm 0.22$ & $2.22 \pm 0.24$ & & 1.3 & M5) & 36) \\
\hline J3420937+2539505 & $16.83 \pm 0.26$ & $16.21 \pm 0.17$ & $0.25=$ & $1.11 \pm$ & 1.4 & \pm 0.18 & 6.1 & & 37) \\
\hline J3420957+2535193 & $15.14 \pm 0.26$ & $14.49 \pm 0.17$ & $0.22 \pm 0.31$ & $1.09 \pm 0.17$ & $1.53 \pm 0.17$ & $1.52 \pm 0.17$ & 6.5 & $\mathrm{~K} 4$ & 1.64( \\
\hline J3420973+2545596 & $16.88 \pm 0.26$ & $16.00 \pm 0.17$ & $0.56 \pm 0.31$ & $1.23 \pm 0.17$ & $1.83=$ & $1.98 \pm 0.18$ & 6.4 & M0 & 1.16( \\
\hline J3420975+2539113 & $18.21 \pm 0.26$ & $17.97 \pm 0.17$ & $-0.26 \pm 0.32$ & $1.30 \pm 0.20$ & & $1.83 \pm$ & 19.4 & & $1.84(0$ \\
\hline J3420988+2519190 & $17.74 \pm 0.26$ & 16.9 & $0.47 \pm 0.31$ & $0.19=$ & 0.84 & $0.84 \pm 0.30$ & 2.5 & G6 & 1.14 \\
\hline J3420995+2531509 & $13.65 \pm 0.26$ & $13.18 \pm 0.17$ & $0.46 \pm 0.31$ & $0.95 \pm 0.17$ & $1.22=$ & $1.26 \pm 0.17$ & 4.2 & $\mathrm{~K} 3($ & $0.01(0.01 . .1 .43)$ \\
\hline $\mathrm{J} 3421083+2526583$ & $18.54 \pm 0.27$ & $17.93 \pm 0.18$ & $0.38 \pm 0.32$ & $1.11=$ & 1.62 & .22 & 18.0 & M3) & 0.8 \\
\hline $\mathrm{J} 3421087+2545497$ & $16.98 \pm 0.26$ & $16.45 \pm 0.17$ & $0.52 \pm 0.31$ & $0.88 \pm 0.18$ & $1.25 \pm 0.19$ & $1.52 \pm 0.20$ & 2.0 & K4 (G8..K6) & $0.05(0.04 . .1 .33)$ \\
\hline $\mathrm{J} 3421116+2540396$ & $19.63 \pm 0.29$ & $18.30 \pm 0.18$ & $0.94 \pm 0.34$ & $1.68 \pm 0.20$ & $2.13 \pm 0.22$ & $2.35 \pm 0.27$ & 8.3 & M3 (M2..M5) & $1.50(0.14 .1 .50)$ \\
\hline $\mathrm{J} 3421128+2529043$ & $18.45 \pm 0.27$ & $17.27 \pm 0.17$ & $0.77 \pm 0.32$ & $0.98 \pm 0.19$ & $1.72 \pm 0.19$ & $1.97 \pm 0.20$ & 1.8 & M0 & 1.54 \\
\hline $\mathrm{J} 3421134+2540588$ & $15.00 \pm 0.26$ & $14.30 \pm 0.17$ & $0.23 \pm 0.31$ & $1.07 \pm 0.17$ & \pm 0.17 & 1.55 & 5.8 & K4 & 1.80 \\
\hline $\mathrm{J} 3421156+2542507$ & $19.65 \pm 0.30$ & $18.23 \pm 0.18$ & $0.97 \pm 0.35$ & $0.86 \pm 0.27$ & $1.59 \pm 0.27$ & $2.17 \pm 0.25$ & 3.7 & M1 (M0..M3) & $1.71(0.80 . .1 .71)$ \\
\hline $\mathrm{J} 3421159+2532185$ & $23.30 \pm 4.53$ & $18.60 \pm 0.19$ & $4.27 \pm 4.54$ & $1.19 \pm 0.28$ & $1.82 \pm 0.28$ & $2.29 \pm 0.30$ & 3.1 & 2..M6) & .03..1.69) \\
\hline $\mathrm{J} 3421170+2537019$ & $15.76 \pm 0.26$ & $15.08 \pm 0.17$ & $0.28 \pm 0.31$ & $1.05 \pm 0.17$ & $1.51 \pm 0.17$ & $1.49 \pm 0.17$ & 5.0 & K4 (K3..M0) & $1.48(0.03 . .1 .48)$ \\
\hline $\mathrm{J} 3421174+2550120$ & $19.34 \pm 0.28$ & $17.76 \pm 0.17$ & $1.29 \pm 0.33$ & $1.41 \pm 0.20$ & $1.97 \pm 0.20$ & $2.19 \pm 0.22$ & 1.5 & M3 (M3..M4) & $1.13(0.08 .1 .22)$ \\
\hline J3421181+2530394 & $16.24 \pm 0.26$ & $15.28 \pm 0.17$ & $0.63 \pm 0.31$ & $1.19 \pm 0.17$ & $1.83 \pm 0.17$ & $1.91 \pm 0.17$ & 4.8 & M0 (M0..M2) & $1.25(0.45 . .1 .46)$ \\
\hline J3421182+2534416 & $17.87 \pm 0.26$ & $16.94 \pm 0.17$ & $0.86 \pm 0.31$ & $1.01 \pm 0.19$ & & & 1. & & 0.27 \\
\hline $\mathrm{J} 3421186+2551108$ & $14.62 \pm 0.26$ & $14.09 \pm 0.17$ & $0.34 \pm 0.31$ & $0.62 \pm 0.17$ & $0.88 \pm 0.17$ & $0.89 \pm 0.17$ & 2.0 & G7 (F8..G9) & $0.71(0.42 . .1 .47)$ \\
\hline J3421199+2535314 & $17.88 \pm 0.26$ & $16.52 \pm 0.17$ & $0.98 \pm 0.31$ & & & & 9. & & \\
\hline $\mathrm{J} 3421209+2542186$ & $18.07 \pm 0.26$ & 17.51 & $0.35 \pm 0.32$ & $0.91 \pm 0.21$ & .23 & $1.59 \pm 0.26$ & 1. & K4 & $0.81(0$ \\
\hline $\mathrm{J} 3421211+2528259$ & & & & & & & 1. & & \\
\hline $\mathrm{J} 3421230+2544315$ & $17.71 \pm 0.26$ & $16.35 \pm 0.17$ & $1.07 \pm 0.31$ & $1.50 \pm 0.17$ & $2.11 \pm 0.17$ & $2.27 \pm 0.17$ & 5. & M3 (M3..M4) & $1.07(0.02 . .1 .28)$ \\
\hline $\mathrm{J} 3421244+2543483$ & $18.78 \pm 0.27$ & & $0.44 \pm 0.32$ & & & & 8 & & \\
\hline $\mathrm{J} 3421253+2529292$ & $16.87 \pm 0.26$ & $16.22 \pm 0.17$ & $0.52 \pm 0.31$ & $0.90 \pm 0.18$ & $1.27 \pm 0.18$ & $1.49 \pm 0.19$ & 2.0 & K4 (K0..K6) & $0.46(0.14 .1 .38)$ \\
\hline $\mathrm{J} 3421259+2530124$ & $16.58 \pm 0.26$ & $15.93 \pm 0.17$ & $0.40 \pm 0.31$ & $1.00 \pm 0.17$ & $1.41 \pm 0.18$ & \pm 0.18 & 3. & K4 (1 & $0.90(0.56 .1 .44)$ \\
\hline $\mathrm{J} 3421285+2551550$ & $17.97 \pm 0.26$ & $17.50 \pm 0.17$ & $0.46 \pm 0.32$ & $0.84 \pm 0.23$ & $1.42 \pm$ & $1.67 \pm$ & 1.0 & 7..K5) & $0.04(0.04 . .1 .14)$ \\
\hline J3421296+2545079 & $18.39 \pm 0.27$ & $17.08 \pm 0.17$ & $1.08 \pm 0.32$ & $1.48 \pm 0.18$ & \pm 0.18 & 2.2 & 4.9 & M3 & $5.1 .26)$ \\
\hline $\mathrm{J} 3421322+2525311$ & $17.83 \pm 0.26$ & $16.93 \pm 0.17$ & $0.59 \pm 0.31$ & $0.70 \pm 0.19$ & $1.38 \pm 0.19$ & $1.60 \pm 0.20$ & 1.7 & K5 (K3..M1) & $1.18(0.03 . .1 .48)$ \\
\hline $\mathrm{J} 3421352+2553189$ & $18.69 \pm 0.27$ & $1742+017$ & $1.01 \pm 0.32$ & & & & 1.0 & M1 & $0.98(0.41 . .1 .49)$ \\
\hline $\mathrm{J} 3421355+2548395$ & $16.47 \pm 0.26$ & $15.79 \pm 0.17$ & $0.40 \pm 0.31$ & $0.95 \pm 0.17$ & $1.40 \pm 0.17$ & $1.49 \pm 0.18$ & 2.1 & K4 (K3..K5) & $1.04(0.73 .1 .44)$ \\
\hline $\mathrm{J} 3421362+2542452$ & $18.38 \pm 0.27$ & $17.83 \pm 0.17$ & $0.21 \pm 0.32$ & $1.03 \pm 0.22$ & $1.27 \pm 0.27$ & $1.85 \pm 0.26$ & 6.8 & K4 (K4..M1) & $1.25(0.24 . .1 .47)$ \\
\hline $\mathrm{J} 3421368+2526402$ & $20.20 \pm 0.34$ & $18.27 \pm 0.18$ & $1.93 \pm 0.39$ & $1.23 \pm 0.27$ & 0.33 & $2.15 \pm 0.38$ & 4.2 & 1..M4) & $0.00(0.00 . .1 .46)$ \\
\hline $\mathrm{J} 3421370+2539192$ & $15.78 \pm 0.26$ & $15.18 \pm 0.17$ & $0.47 \pm 0.31$ & $0.91 \pm 0.17$ & $1.40 \pm 0.17$ & & 1. & K4 (K0..K6) & $0.50(0.14 . .1 .31)$ \\
\hline $\mathrm{J} 3421376+2530584$ & $18.74 \pm 0.27$ & 17.73 & $0.71 \pm 0.32$ & 1. & 1.8 & $1.73 \pm 0.26$ & 4.6 & M0 & $1.15(0$ \\
\hline $\mathrm{J} 342$ & & & & & & & 1.7 & & (..1.46) \\
\hline J34214 & 16 & & $4 \pm 0.31$ & & & & 1.7 & K4 & 37) \\
\hline $\mathrm{J} 3421456+2543054$ & 17.6 & 17.2 & $0.18 \pm 0.31$ & & 1. & 1.74 & 8.0 & ..K6) & $0.57(0$ \\
\hline J34214 & & & & & & & 1.1 & & \\
\hline $\mathrm{J} 3421473+2539574$ & $15.26 \pm 0.26$ & 14.71 & $0.27 \pm 0.31$ & 1. & 1. & 17 & 4.8 & K4 ( & $1.04(\mathrm{C})$ \\
\hline $\mathrm{J} 3421478+2540144$ & 0.26 & 17. & 0. & 0. & & & 1.8 & 6) & 42) \\
\hline $\mathrm{J} 3421508+2539338$ & $19.27 \pm 0.28$ & & $-0.14 \pm 0.34$ & & & .34 & 29.3 & M0 & 1..1.45) \\
\hline J34215 & 17. & & & & & & 4.3 & M2) & \\
\hline $\mathrm{J} 3421513+2548481$ & $15.69 \pm 0.26$ & $15.04 \pm 0.17$ & $0.43 \pm 0.31$ & \pm 0.17 & $=0.17$ & 17 & 2.3 & K4 (K3..K6) & $0.79(0.50 . .1 .26)$ \\
\hline $\mathrm{J} 3421519+2542225$ & & & & & & & 22.1 & & \\
\hline $\mathrm{J} 3421539+2530576$ & $16.29 \pm 0.26$ & $15.70 \pm 0.17$ & $0.49 \pm 0.31$ & $0.93 \pm 0.17$ & $1.35 \pm 0.18$ & $1.44 \pm 0.18$ & 1.9 & K4 (K0..K7) & $0.40(0.03 .1 .40)$ \\
\hline J3421557+2538368 & $18.39 \pm 0.27$ & $17.76 \pm 0.17$ & $0.35 \pm 0.32$ & & & & 2.0 & K4 (K4..M0) & $1.04(0.01 . .1 .28)$ \\
\hline $\mathrm{J} 3421559+2541544$ & $19.94 \pm 0.32$ & & $1.22 \pm 0$ & $2.18 \pm 0.22$ & & $3.06 \pm$ & 13.9 & M5 & $.1 .18)$ \\
\hline+2543062 & 16.6 & & & & & & 3.2 & $\mathrm{~K} 3$ & \\
\hline $\mathrm{J} 3421577+2522258$ & $15.20 \pm 0.26$ & $14.34 \pm 0.17$ & $0.84 \pm 0.31$ & $1.09 \pm 0.17$ & $1.72 \pm 0$ & $1.81 \pm 0.17$ & 1.6 & M0 (K4..M0) & $0.06(0.06 . .1 .11)$ \\
\hline & & & & & & & 1.4 & & \\
\hline $\mathrm{J} 3421601+2523437$ & $15.08 \pm 0.26$ & $14.32 \pm 0.17$ & $0.41 \pm 0.31$ & $0.69 \pm 0.17$ & $1.01 \pm 0.17$ & $1.03 \pm 0.17$ & 2.0 & K0 (G8...K7) & $1.26(0.06 .1 .44)$ \\
\hline $\mathrm{J} 3421608+2552147$ & & & $0.48 \pm 0$ & & & & 1.7 & K3 (K2..K7) & $0.86(0.24 .1 .41)$ \\
\hline $\mathrm{J} 3421618+2547371$ & $15.45 \pm 0.26$ & $14.81 \pm 0.17$ & $0.50 \pm 0.31$ & $0.94 \pm 0.17$ & $1.36 \pm 0.17$ & $1.41 \pm 0.17$ & 2.1 & K4 (K2..K7) & $0.54(0.13 . .1 .02)$ \\
\hline-2551342 & $19.02 \pm 0$ & & $1.72 \pm 0$ & & & & 2.3 & M5 (M3..M5) & $0.00(0.00 . .1 .47)$ \\
\hline $\mathrm{J} 3421646+2531470$ & $17.25 \pm 0.26$ & $16.56 \pm 0.17$ & $0.39 \pm 0.31$ & $1.33 \pm 0.17$ & $1.91 \pm 0.18$ & $2.07 \pm 0.18$ & 12.8 & M0 (K5...M2) & $1.10(0.16$. \\
\hline $\mathrm{J} 3421665+2538348$ & $17.38 \pm 0.26$ & $16.84 \pm 0.17$ & $0.44 \pm 0.31$ & $1.04 \pm 0.18$ & $1.28 \pm 0.21$ & $1.47 \pm 0.23$ & 3.7 & K4 (G9..K4) & $0.41(0.41 . .1 .33)$ \\
\hline
\end{tabular}




\begin{tabular}{|c|c|c|c|c|c|c|c|c|c|}
\hline \multirow[b]{2}{*}{ 2MASS-ID } & \multicolumn{2}{|c|}{ measured magnitudes } & & extinction $\mathrm{c}$ & ected colors & & & & \\
\hline & Rmag & Imag & R-I & I-J & $\mathrm{I}-\mathrm{H}$ & $\mathrm{I}-\mathrm{K}_{s}$ & $\chi^{2}$ & SpT & $\mathrm{Av}$ \\
\hline J3421666+2541405 & $20.19 \pm 0.33$ & $18.36 \pm 0.18$ & $1.49 \pm 0.38$ & $1.62 \pm 0.22$ & $2.22 \pm 0.23$ & $2.42 \pm 0.25$ & 1.7 & M4 (M4..M5) & $1.27(0.58 . .1 .39)$ \\
\hline $\mathrm{J} 3421678+2527505$ & $19.70 \pm 0.30$ & $18.47 \pm 0.19$ & $0.83 \pm 0.35$ & $1.33 \pm 0.25$ & $2.46 \pm 0.23$ & $2.91 \pm \ldots$ & 11.1 & M3 (M1..M5) & $1.50(0.21 . .1 .50)$ \\
\hline $\mathrm{J} 3421687+2524447$ & $15.25 \pm 0.26$ & $14.48 \pm 0.17$ & $0.53 \pm 0.31$ & $0.90 \pm 0.17$ & $1.35 \pm 0.17$ & $1.41 \pm 0.17$ & 1.7 & $\mathrm{~K} 4(\mathrm{~K} 1 . . \mathrm{K} 7)$ & $0.88(0.50 . .1 .43)$ \\
\hline $\mathrm{J} 3421688+2536073$ & $17.49 \pm 0.26$ & $16.86 \pm 0.17$ & $0.52 \pm 0.31$ & $1.06 \pm 0.18$ & $1.31 \pm 0.20$ & $1.27 \pm 0.25$ & 5.5 & $\mathrm{~K} 4(\mathrm{~K} 0 . . \mathrm{K} 5)$ & $0.41(0.12 . .1 .47)$ \\
\hline $\mathrm{J} 3421689+2553488$ & $19.56 \pm 0.26$ & $18.01 \pm 0.17$ & $1.46 \pm 0.32$ & $1.35 \pm 0.23$ & $1.74 \pm 0.28$ & $2.15 \pm 0.29$ & 1.9 & M3 (M1..M3) & $0.35(0.11 . .1 .47)$ \\
\hline $\mathrm{J} 3421694+2526457$ & $17.55 \pm 0.26$ & $17.05 \pm 0.17$ & $0.19 \pm 0.31$ & $0.65 \pm 0.20$ & $0.50 \pm 0.27$ & $1.19 \pm \ldots$ & 4.6 & F7 (A6..K3) & $1.17(0.22 . .1 .50)$ \\
\hline $\mathrm{J} 3421710+2528554$ & $16.94 \pm 0.26$ & $16.44 \pm 0.17$ & $0.35 \pm 0.31$ & $0.60 \pm 0.18$ & $0.98 \pm 0.20$ & $1.20 \pm 0.21$ & 1.4 & $\mathrm{~K} 0$ (G6..K2) & $0.56(0.15 . .1 .13)$ \\
\hline $\mathrm{J} 3421713+2551010$ & $15.60 \pm 0.26$ & $15.00 \pm 0.17$ & $0.30 \pm 0.31$ & $0.31 \pm 0.17$ & $0.55 \pm 0.18$ & $0.48 \pm 0.18$ & 1.3 & F5 (F5..G9) & $1.13(0.18 . .1 .13)$ \\
\hline $\mathrm{J} 3421745+2547528$ & $13.49 \pm 0.26$ & $12.85 \pm 0.17$ & $0.48 \pm 0.31$ & $0.92 \pm 0.17$ & $1.40 \pm 0.17$ & $1.42 \pm 0.17$ & 1.9 & $\mathrm{~K} 4(\mathrm{~K} 0 . . \mathrm{K} 6)$ & $0.62(0.41 . .1 .41)$ \\
\hline $\mathrm{J} 3421762+2553344$ & $18.35 \pm 0.26$ & $17.51 \pm 0.17$ & $0.45 \pm 0.31$ & $0.60 \pm 0.23$ & $1.81 \pm \ldots$ & $1.67 \pm 0.24$ & 3.3 & $\mathrm{~K} 4$ (K3...M1) & $1.47(0.31 . .1 .47)$ \\
\hline $\mathrm{J} 3421778+2531087$ & $17.40 \pm 0.26$ & $16.73 \pm 0.17$ & $0.50 \pm 0.31$ & $1.05 \pm 0.18$ & $1.37 \pm 0.19$ & $1.27 \pm 0.23$ & 5.2 & $\mathrm{~K} 4(\mathrm{~K} 0 . . \mathrm{K} 6)$ & $0.61(0.12 . .1 .48)$ \\
\hline $\mathrm{J} 3421779+2522063$ & $17.27 \pm 0.26$ & $16.01 \pm 0.17$ & $1.14 \pm 0.31$ & $1.11 \pm 0.17$ & $1.81 \pm 0.18$ & $1.96 \pm 0.18$ & 1.1 & M2 (M0..M2) & $0.46(0.27 . .0 .97)$ \\
\hline $\mathrm{J} 3421836+2549579$ & $16.85 \pm 0.26$ & $16.39 \pm 0.17$ & $0.40 \pm 0.31$ & $0.97 \pm 0.18$ & $1.45 \pm 0.18$ & $1.43 \pm 0.20$ & 2.5 & K4 (K0..K6) & $0.22(0.02 . .1 .14)$ \\
\hline $\mathrm{J} 3421862+2543418$ & $17.66 \pm 0.26$ & $16.65 \pm 0.17$ & $0.67 \pm 0.31$ & $1.24 \pm 0.18$ & $1.82 \pm 0.18$ & $1.85 \pm 0.18$ & 4.8 & M0 (M0..M3) & $1.30(0.28 . .1 .30)$ \\
\hline $\mathrm{J} 3421871+2543584$ & $19.76 \pm 0.31$ & $18.33 \pm 0.18$ & $1.17 \pm 0.35$ & $1.43 \pm 0.24$ & $1.95 \pm 0.26$ & $2.39 \pm 0.27$ & 2.5 & M3 (M2..M4) & $0.98(0.32 . .1 .45)$ \\
\hline $\mathrm{J} 3421874+2543115$ & $14.53 \pm 0.26$ & $13.90 \pm 0.17$ & $0.34 \pm 0.31$ & $1.05 \pm 0.17$ & $1.45 \pm 0.17$ & $1.47 \pm 0.17$ & 4.2 & K4 (K3..M0) & $1.09(0.16 . .1 .28)$ \\
\hline $\mathrm{J} 3421875+2521377$ & $17.88 \pm 0.26$ & $17.44 \pm 0.17$ & $0.13 \pm 0.32$ & $1.14 \pm 0.19$ & $1.76 \pm 0.20$ & $1.35 \pm 0.27$ & 11.5 & K4 (K3...M0) & $1.16(0.35 . .1 .40)$ \\
\hline $\mathrm{J} 3421880+2538271$ & $16.82 \pm 0.26$ & $16.22 \pm 0.17$ & $0.27 \pm 0.31$ & $1.06 \pm 0.18$ & $1.48 \pm 0.18$ & $1.52 \pm 0.18$ & 4.8 & K4 (K3..M1) & $1.21(0.05 . .1 .42)$ \\
\hline $\mathrm{J} 3421882+2551488$ & $20.10 \pm 0.33$ & $18.61 \pm 0.19$ & $1.20 \pm 0.38$ & $1.74 \pm 0.24$ & $2.44 \pm 0.25$ & $2.57 \pm 0.29$ & 5.8 & M4 (M3..M5) & $1.06(0.26 . .1 .32)$ \\
\hline $\mathrm{J} 3421902+2522237$ & $15.96 \pm 0.26$ & $15.30 \pm 0.17$ & $0.33 \pm 0.31$ & $0.46 \pm 0.17$ & $0.64 \pm 0.18$ & $0.62 \pm 0.18$ & 1.8 & F7 (F7..G8) & $1.28(0.65 . .1 .46)$ \\
\hline $\mathrm{J} 3421905+2549231$ & $17.14 \pm 0.26$ & $16.60 \pm 0.17$ & $0.54 \pm 0.31$ & $0.86 \pm 0.18$ & $1.29 \pm 0.19$ & $1.03 \pm 0.26$ & 4.4 & $\mathrm{~K} 3(\mathrm{~F} 8 . . \mathrm{K} 3)$ & $0.01(0.01 . .1 .42)$ \\
\hline $\mathrm{J} 3421920+2524066$ & $15.91 \pm 0.26$ & $15.19 \pm 0.17$ & $0.53 \pm 0.31$ & $0.94 \pm 0.17$ & $1.34 \pm 0.17$ & $1.38 \pm 0.17$ & 2.5 & $\mathrm{~K} 4(\mathrm{~K} 0 . . \mathrm{K} 7)$ & $0.69(0.25 . .1 .42)$ \\
\hline $\mathrm{J} 3421926+2553208$ & $15.91 \pm 0.26$ & $15.34 \pm 0.17$ & $0.35 \pm 0.31$ & $0.65 \pm 0.17$ & $0.82 \pm 0.18$ & $0.89 \pm 0.18$ & 2.5 & G7 (F5..K3) & $0.82(0.15 . .1 .45)$ \\
\hline $\mathrm{J} 3421947+2547469$ & $17.39 \pm 0.26$ & $16.91 \pm 0.17$ & $0.47 \pm 0.31$ & $0.93 \pm 0.19$ & $1.17 \pm 0.22$ & $1.61 \pm 0.23$ & 3.6 & $\mathrm{~K} 4(\mathrm{G} 2 . . \mathrm{K} 4)$ & $0.06(0.06 . .1 .46)$ \\
\hline $\mathrm{J} 3421960+2541490$ & $18.58 \pm 0.27$ & $17.74 \pm 0.17$ & $0.49 \pm 0.32$ & $0.80 \pm 0.23$ & $1.42 \pm 0.24$ & $1.44 \pm 0.30$ & 1.1 & $\mathrm{~K} 4$ (K4..M1) & $1.32(0.01 . .1 .41)$ \\
\hline $\mathrm{J} 3421969+2536453$ & $18.21 \pm 0.26$ & $17.58 \pm 0.17$ & $0.34 \pm 0.32$ & $1.04 \pm 0.20$ & $1.21 \pm 0.25$ & $1.68 \pm 0.25$ & 4.9 & K4 (K3..M1) & $1.09(0.04 . .1 .28)$ \\
\hline $\mathrm{J} 3421976+2540553$ & $20.27 \pm 0.33$ & $18.98 \pm 0.20$ & $1.14 \pm 0.39$ & $2.34 \pm 0.24$ & $2.79 \pm 0.28$ & $3.10 \pm 0.29$ & 16.3 & M5 (M4..M6.5) & $0.58(0.10 . .1 .45)$ \\
\hline $\mathrm{J} 3421988+2544153$ & $17.14 \pm 0.26$ & $16.61 \pm 0.17$ & $0.33 \pm 0.31$ & $-0.33 \pm 0.23$ & $0.19 \pm 0.30$ & $0.54 \pm 0.29$ & 8.0 & F0 (A3..G9) & $0.74(0.01 . .1 .44)$ \\
\hline $\mathrm{J} 3422002+2531085$ & $20.52 \pm 0.39$ & $18.55 \pm 0.19$ & $1.66 \pm 0.43$ & $1.33 \pm 0.27$ & $2.14 \pm 0.27$ & $2.47 \pm 0.29$ & 1.7 & M4 (M3..M5) & $1.17(0.05 . .1 .45)$ \\
\hline $\mathrm{J} 3422004+2544254$ & $16.60 \pm 0.26$ & $15.96 \pm 0.17$ & $0.49 \pm 0.31$ & $1.01 \pm 0.17$ & $1.35 \pm 0.18$ & $1.38 \pm 0.18$ & 3.6 & $\mathrm{~K} 4$ (K1..K6) & $0.57(0.16 . .1 .37)$ \\
\hline $\mathrm{J} 3422044+2550297$ & $17.63 \pm 0.26$ & $17.12 \pm 0.17$ & $0.46 \pm 0.31$ & $0.57 \pm 0.22$ & $1.39 \pm 0.22$ & $1.14 \pm$ & 2.3 & K3 (G5..K4) & $0.17(0.17 . .1 .44)$ \\
\hline $\mathrm{J} 3422071+2526364$ & $18.27 \pm 0.27$ & $17.20 \pm 0.17$ & $0.74 \pm 0.32$ & $1.06 \pm 0.19$ & $1.78 \pm 0.19$ & $1.92 \pm 0.20$ & 2.0 & Mo (M0..M1) & $1.26(0.78 . .1 .46)$ \\
\hline $\mathrm{J} 3422077+2545008$ & $17.81 \pm 0.26$ & $17.38 \pm 0.17$ & $0.18 \pm 0.31$ & $1.05 \pm 0.20$ & $1.50 \pm 0.21$ & $1.68 \pm 0.25$ & 5.2 & $\mathrm{~K} 4$ (K1..K7) & $0.92(0.34 \ldots 1.41)$ \\
\hline $\mathrm{J} 3422085+2545254$ & $18.71 \pm 0.27$ & $17.64 \pm 0.17$ & $0.92 \pm 0.32$ & $1.20 \pm 0.21$ & $1.71 \pm 0.22$ & $1.95 \pm 0.27$ & 1.8 & M1 (M0..M2) & $0.58(0.39 . .0 .91)$ \\
\hline $\mathrm{J} 3422096+2533546$ & $16.98 \pm 0.26$ & $16.27 \pm 0.17$ & $0.36 \pm 0.31$ & $0.98 \pm 0.18$ & $1.43 \pm 0.18$ & $1.49 \pm 0.18$ & 2.7 & K4 (K3..M1) & $1.34(0.02 . .1 .49)$ \\
\hline $\mathrm{J} 3422109+2522485$ & $17.28 \pm 0.26$ & $16.54 \pm 0.17$ & $0.30 \pm 0.31$ & $0.34 \pm 0.18$ & $0.35 \pm 0.22$ & $0.44 \pm 0.25$ & 1.9 & F4 (F3..G7) & $1.66(0.75 . .1 .67)$ \\
\hline $\mathrm{J} 3422130+2551592$ & $18.29 \pm 0.27$ & $16.76 \pm 0.17$ & $1.43 \pm 0.32$ & $1.28 \pm 0.18$ & $1.87 \pm 0.18$ & $2.17 \pm 0.18$ & 1.2 & M3 (M2..M3) & $0.37(0.13 . .1 .15)$ \\
\hline $\mathrm{J} 3422135+2543321$ & $14.85 \pm 0.26$ & $14.37 \pm 0.17$ & $0.44 \pm 0.31$ & $1.00 \pm 0.17$ & $1.36 \pm 0.17$ & $1.43 \pm 0.17$ & 3.1 & $\mathrm{~K} 4$ (G7..K5) & $0.11(0.11 . .1 .23)$ \\
\hline $\mathrm{J} 3422149+2543573$ & $17.52 \pm 0.26$ & $17.05 \pm 0.17$ & $0.40 \pm 0.31$ & $1.04 \pm 0.19$ & $1.28 \pm 0.22$ & $1.53 \pm 0.24$ & 3.7 & K4 (G7..K5) & $0.25(0.01 . .1 .46)$ \\
\hline $\mathrm{J} 3422167+2529165$ & $18.65 \pm 0.27$ & $17.49 \pm 0.17$ & $1.15 \pm 0.32$ & $1.08 \pm 0.21$ & $1.53 \pm 0.24$ & $1.65 \pm 0.30$ & 3.0 & M1 (K4..M2) & $0.00(0.00 . .1 .47)$ \\
\hline $\mathrm{J} 3422176+2531189$ & $18.96 \pm 0.27$ & $17.74 \pm 0.17$ & $0.82 \pm 0.32$ & $0.93 \pm 0.22$ & $1.67 \pm 0.22$ & $2.29 \pm 0.22$ & 6.9 & M1 (M0..M3) & $1.50(0.09 . .1 .50)$ \\
\hline $\mathrm{J} 3422179+2520431$ & $17.90 \pm 0.27$ & $16.74 \pm 0.17$ & $0.57 \pm 0.32$ & $0.19 \pm 0.19$ & $0.56 \pm 0.22$ & $0.56 \pm 0.24$ & 2.8 & G0 (F7..G8) & $2.19(1.65 . .2 .19)$ \\
\hline $\mathrm{J} 3422181+2525176$ & $16.16 \pm 0.26$ & $15.51 \pm 0.17$ & $0.34 \pm 0.31$ & $0.66 \pm 0.17$ & $0.95 \pm 0.18$ & $0.80 \pm 0.18$ & 3.6 & G7 (G2..K2) & $1.17(0.36 . .1 .48)$ \\
\hline $\mathrm{J} 3422184+2533470$ & $18.37 \pm 0.27$ & $17.61 \pm 0.17$ & $0.48 \pm 0.32$ & $0.85 \pm 0.22$ & $1.25 \pm 0.25$ & $1.59 \pm 0.27$ & 1.7 & $\mathrm{~K} 4(\mathrm{~K} 2 . . \mathrm{K} 7)$ & $1.06(0.40 . .1 .49)$ \\
\hline $\mathrm{J} 3422193+2525327$ & $18.61 \pm 0.27$ & $16.98 \pm 0.17$ & $1.32 \pm 0.32$ & $0.09 \pm 0.22$ & $0.91 \pm 0.22$ & $1.61 \pm 0.21$ & 23.5 & K7 (K4..M1) & $1.14(0.07 . .1 .41)$ \\
\hline $\mathrm{J} 3422195+2538389$ & $16.88 \pm 0.26$ & $16.06 \pm 0.17$ & $0.52 \pm 0.31$ & $1.34 \pm 0.17$ & $1.88 \pm 0.18$ & $1.92 \pm 0.18$ & 9.5 & M0 (K6..M2) & $1.11(0.06 . .1 .45)$ \\
\hline $\mathrm{J} 3422239+2538150$ & $15.56 \pm 0.26$ & $15.11 \pm 0.17$ & $0.41 \pm 0.31$ & $1.03 \pm 0.17$ & $1.38 \pm 0.17$ & $1.44 \pm 0.18$ & 3.5 & K4 (K0..K6) & $0.18(0.00 . .1 .18)$ \\
\hline $\mathrm{J} 3422241+2526293$ & $14.35 \pm 0.26$ & $13.75 \pm 0.17$ & $0.37 \pm 0.31$ & $0.77 \pm 0.17$ & $1.00 \pm 0.17$ & $1.05 \pm 0.17$ & 2.9 & $\mathrm{~K} 0(\mathrm{G} 3 . . \mathrm{K} 2)$ & $0.87(0.49 . .1 .50)$ \\
\hline $\mathrm{J} 3422244+2543112$ & $18.31 \pm 0.27$ & $17.59 \pm 0.17$ & $0.47 \pm 0.32$ & $1.34 \pm 0.19$ & $1.79 \pm 0.21$ & $2.11 \pm 0.22$ & 9.4 & M0 (K6..M3) & $0.95(0.21 . .1 .42)$ \\
\hline $\mathrm{J} 3422259+2553353$ & $18.21 \pm 0.26$ & $17.16 \pm 0.17$ & $0.95 \pm 0.31$ & $0.54 \pm 0.22$ & $1.38 \pm 0.22$ & $2.23 \pm \ldots$ & 2.7 & K7 (K1..K7) & $0.38(0.05 . .1 .47)$ \\
\hline $\mathrm{J} 3422266+2528539$ & $18.41 \pm 0.27$ & $17.00 \pm 0.17$ & $1.36 \pm 0.32$ & $1.72 \pm 0.18$ & $2.30 \pm 0.18$ & $2.46 \pm 0.19$ & 4.8 & M4 (M2..M4) & $0.16(0.16 . .1 .46)$ \\
\hline $\mathrm{J} 3422306+2545597$ & $17.94 \pm 0.26$ & $17.39 \pm 0.17$ & $0.51 \pm 0.32$ & $1.11 \pm 0.20$ & $0.94 \pm 0.34$ & $1.39 \pm 0.33$ & 7.6 & K4 (F7..K7) & $0.16(0.16 . .1 .39)$ \\
\hline $\mathrm{J} 3422324+2530525$ & $17.81 \pm 0.26$ & $17.19 \pm 0.17$ & $0.15 \pm 0.31$ & $1.08 \pm 0.18$ & $1.56 \pm 0.20$ & $1.61 \pm 0.21$ & 6.7 & K4 (K4..M1) & $1.76(0.36 . .1 .76)$ \\
\hline $\mathrm{J} 3422328+2546226$ & $15.38 \pm 0.26$ & $14.84 \pm 0.17$ & $0.47 \pm 0.31$ & $0.97 \pm 0.17$ & $1.35 \pm 0.17$ & $1.44 \pm 0.17$ & 2.4 & K4 (G7..K6) & $0.28(0.07 . .1 .45)$ \\
\hline $\mathrm{J} 3422340+2552536$ & $18.76 \pm 0.26$ & $17.97 \pm 0.18$ & $0.58 \pm 0.32$ & $0.70 \pm 0.29$ & $1.24 \pm 0.34$ & $1.75 \pm 0.33$ & 2.5 & K5 (K0..M0) & $0.79(0.10 . .1 .39)$ \\
\hline $\mathrm{J} 3422348+2520539$ & $19.03 \pm 0.28$ & $17.64 \pm 0.17$ & $1.14 \pm 0.33$ & $1.12 \pm 0.20$ & $1.74 \pm 0.22$ & $2.02 \pm 0.23$ & 1.1 & M2 (M0..M3) & $0.95(0.17 . .1 .43)$ \\
\hline $\mathrm{J} 3422360+2523418$ & $15.50 \pm 0.26$ & $14.80 \pm 0.17$ & $0.37 \pm 0.31$ & $0.62 \pm 0.17$ & $0.88 \pm 0.17$ & $0.85 \pm 0.17$ & 2.3 & G7 (G1..K4) & $1.21(0.09 . .1 .47)$ \\
\hline $\mathrm{J} 3422362+2548180$ & $17.32 \pm 0.26$ & $16.39 \pm 0.17$ & $0.85 \pm 0.31$ & $0.65 \pm 0.19$ & $1.29 \pm 0.20$ & $1.64 \pm 0.20$ & 2.6 & K7 (K0..K7) & $0.28(0.16 . .1 .26)$ \\
\hline $\mathrm{J} 3422363+2519232$ & $17.31 \pm 0.26$ & $16.43 \pm 0.17$ & $0.46 \pm 0.31$ & $0.54 \pm 0.18$ & $0.74 \pm 0.20$ & $0.89 \pm 0.21$ & 2.0 & G7 (G7..K7) & $1.61(0.05 . .1 .63)$ \\
\hline $\mathrm{J} 3422383+2550338$ & $19.11 \pm 0.27$ & $17.48 \pm 0.17$ & $1.55 \pm 0.32$ & $1.29 \pm 0.19$ & $1.85 \pm 0.21$ & $1.98 \pm 0.24$ & 2.5 & M3 (M2..M3) & $0.32(0.07 . .1 .27)$ \\
\hline $\mathrm{J} 3422386+2535272$ & $18.11 \pm 0.26$ & $17.66 \pm 0.17$ & $0.18 \pm 0.32$ & $1.14 \pm 0.21$ & $1.49 \pm 0.25$ & $1.63 \pm 0.27$ & 5.8 & $\mathrm{~K} 4(\mathrm{~K} 2 . . \mathrm{K} 7)$ & $0.99(0.43 . .1 .49)$ \\
\hline $\mathrm{J} 3422398+2550541$ & $16.88 \pm 0.26$ & $16.17 \pm 0.17$ & $0.31 \pm 0.31$ & $0.12 \pm 0.18$ & $0.24 \pm 0.20$ & $0.07 \pm 0.29$ & 1.9 & A9 (A5..G2) & $1.53(0.35 . .1 .56)$ \\
\hline $\mathrm{J} 3422420+2547216$ & $18.95 \pm 0.27$ & $17.96 \pm 0.18$ & $0.66 \pm 0.32$ & $1.07 \pm 0.22$ & $1.89 \pm 0.23$ & $1.97 \pm 0.27$ & 3.1 & M0 (M0..M2) & $1.24(0.53 . .1 .32)$ \\
\hline $\mathrm{J} 3422424+2553547$ & $15.82 \pm 0.26$ & $15.15 \pm 0.17$ & $0.38 \pm 0.31$ & $0.60 \pm 0.17$ & $0.89 \pm 0.17$ & $0.83 \pm 0.18$ & 2.2 & G7 (F7..G9) & $1.12(1.08 . .1 .48)$ \\
\hline $\mathrm{J} 3422447+2523303$ & $15.31 \pm 0.26$ & $14.60 \pm 0.17$ & $0.32 \pm 0.31$ & $0.55 \pm 0.17$ & $0.77 \pm 0.17$ & $0.69 \pm 0.17$ & 2.5 & G4 (G4..K3) & $1.47(0.02 . .1 .47)$ \\
\hline $\mathrm{J} 3422461+2550569$ & $17.03 \pm 0.26$ & $16.39 \pm 0.17$ & $0.03 \pm 0.31$ & $-0.60 \pm 0.21$ & $-0.64 \pm 0.30$ & $-0.98 \pm \ldots$ & 2.0 & B0 (B0..B7) & $2.31(1.40 . .2 .31)$ \\
\hline $\mathrm{J} 3422501+2536449$ & $18.14 \pm 0.26$ & $17.73 \pm 0.17$ & $0.01 \pm 0.32$ & $1.03 \pm 0.22$ & $1.75 \pm 0.21$ & $1.88 \pm$ & 8.4 & K4 (K3..M0) & $1.47(0.04 . .1 .47)$ \\
\hline $\mathrm{J} 3422504+2537449$ & $15.00 \pm 0.26$ & $14.53 \pm 0.17$ & $0.35 \pm 0.31$ & $1.07 \pm 0.17$ & $1.41 \pm 0.17$ & $1.47 \pm 0.17$ & 4.6 & $\mathrm{~K} 4(\mathrm{~K} 0 . . \mathrm{K} 4)$ & $0.44(0.16 . .1 .27)$ \\
\hline $\mathrm{J} 3422532+2545164$ & $17.57 \pm 0.26$ & $17.09 \pm 0.17$ & $0.43 \pm 0.31$ & $0.92 \pm 0.19$ & $1.36 \pm 0.22$ & $1.40 \pm$ & 1.5 & K4 (K0..K6) & $0.20(0.08 . .0 .82)$ \\
\hline $\mathrm{J} 3422551+2530158$ & $18.30 \pm 0.27$ & $17.39 \pm 0.17$ & $0.92 \pm 0.32$ & $0.36 \pm 0.27$ & $1.45 \pm 0.24$ & $1.35 \pm 0.33$ & 4.9 & K7 (F9..K7) & $0.00(0.00 . .1 .46)$ \\
\hline $\mathrm{J} 3422562+2540357$ & $16.84 \pm 0.26$ & $16.21 \pm 0.17$ & $0.27 \pm 0.31$ & $1.09 \pm 0.17$ & $1.47 \pm 0.18$ & $1.51 \pm 0.18$ & 5.4 & K4 (K4..M0) & $1.37(0.01 . .1 .50)$ \\
\hline $\mathrm{J} 3422589+2520049$ & $18.24 \pm 0.27$ & $17.15 \pm 0.17$ & $0.59 \pm 0.32$ & $0.14 \pm 0.22$ & $0.43 \pm$ & $0.81 \pm$ & 2.4 & G0 (F0..K6) & $1.85(0.30 . .1 .85)$ \\
\hline $\mathrm{J} 3422613+2524293$ & $16.51 \pm 0.26$ & $15.81 \pm 0.17$ & $0.49 \pm 0.31$ & $0.85 \pm 0.17$ & $1.25 \pm 0.18$ & $1.26 \pm 0.18$ & 2.2 & K3 (G9..K7) & $0.81(0.18 . .1 .47)$ \\
\hline $\mathrm{J} 3422640+2527382$ & $16.62 \pm 0.26$ & $15.95 \pm 0.17$ & $0.39 \pm 0.31$ & $0.76 \pm 0.17$ & $0.97 \pm 0.18$ & $1.04 \pm 0.19$ & 2.9 & $\mathrm{~K} 0(\mathrm{G} 6 . . \mathrm{K} 7)$ & $1.05(0.02 . .1 .25)$ \\
\hline $\mathrm{J} 3422695+2527563$ & $19.33 \pm 0.28$ & $18.03 \pm 0.18$ & $0.86 \pm 0.33$ & $1.23 \pm 0.21$ & $1.48 \pm 0.26$ & $2.17 \pm 0.24$ & 5.5 & M1 (M0..M3) & $1.64(0.25 . .1 .64)$ \\
\hline $\mathrm{J} 3422702+2550135$ & $17.67 \pm 0.26$ & $17.15 \pm 0.17$ & $0.48 \pm 0.31$ & $0.78 \pm 0.20$ & $1.21 \pm 0.24$ & $1.43 \pm$ & 1.2 & K3 (G6..K4) & $0.14(0.02 . .1 .29)$ \\
\hline $\mathrm{J} 3422703+2525168$ & $17.97 \pm 0.26$ & $16.70 \pm 0.17$ & $1.26 \pm 0.31$ & $1.03 \pm 0.18$ & $1.67 \pm 0.19$ & $1.98 \pm 0.20$ & 1.7 & M2 (K7..M2) & $0.06(0.06 . .1 .28)$ \\
\hline $\mathrm{J} 3422717+2542481$ & $17.40 \pm 0.26$ & $16.80 \pm 0.17$ & $0.47 \pm 0.31$ & $0.93 \pm 0.18$ & $1.46 \pm 0.19$ & $1.32 \pm 0.24$ & 2.7 & K4 (K1..K5) & $0.49(0.13 . .1 .36)$ \\
\hline $\mathrm{J} 3422736+2523174$ & $17.36 \pm 0.26$ & $16.60 \pm 0.17$ & $0.33 \pm 0.31$ & $0.44 \pm 0.19$ & $0.43 \pm 0.22$ & $0.39 \pm 0.28$ & 2.9 & F5 (F4..K1) & $1.63(0.31 . .1 .64)$ \\
\hline $\mathrm{J} 3422757+2526249$ & $16.74 \pm 0.26$ & $15.98 \pm 0.17$ & $0.41 \pm 0.31$ & $0.93 \pm 0.17$ & $1.47 \pm 0.18$ & $1.44 \pm 0.18$ & 2.3 & K4 (K3..M1) & $1.34(0.01 . .1 .48)$ \\
\hline $\mathrm{J} 3422772+2522493$ & $14.96 \pm 0.26$ & $14.00 \pm 0.17$ & $0.50 \pm 0.31$ & $-1.29 \pm 0.18$ & $-0.77 \pm 0.18$ & $-0.77 \pm 0.18$ & 37.7 & B0 (B0..A7) & $1.75(0.03 . .1 .75)$ \\
\hline $\mathrm{J} 3422783+2541464$ & $17.79 \pm 0.26$ & $16.98 \pm 0.17$ & $0.45 \pm 0.31$ & $1.35 \pm 0.18$ & $1.82 \pm 0.18$ & $2.07 \pm 0.19$ & 10.9 & M0 (M0..M3) & $1.35(0.22 . .1 .35)$ \\
\hline
\end{tabular}




\begin{tabular}{|c|c|c|c|c|c|c|c|c|c|}
\hline \multirow[b]{2}{*}{ 2MASS-ID } & \multicolumn{2}{|c|}{ measured magnitudes } & & extinction $\mathrm{cc}$ & ected colors & & & & \\
\hline & Rmag & Imag & R-I & 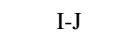 & 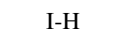 & $\mathrm{I}-\mathrm{K}_{s}$ & 2 & SpT & $\mathrm{Av}$ \\
\hline $\mathrm{J} 3422827+2551145$ & $15.15 \pm 0.26$ & $14.42 \pm 0.17$ & $0.72 \pm 0.31$ & $-1.54 \pm 0.19$ & $-0.98 \pm 0.20$ & $-1.22 \pm 0.28$ & 53.8 & B0 (B0..B7) & $0.00(0.00 . .1 .49)$ \\
\hline $\mathrm{J} 3422832+2522400$ & $14.69 \pm 0.26$ & $13.84 \pm 0.17$ & $0.51 \pm 0.31$ & $0.80 \pm 0.17$ & $1.24 \pm 0.17$ & $1.28 \pm 0.17$ & 1.6 & K3 (K1..M0) & $1.25(0.01 . .1 .50)$ \\
\hline $\mathrm{J} 3422833+2547538$ & $15.33 \pm 0.26$ & $14.81 \pm 0.17$ & $0.36 \pm 0.31$ & $0.65 \pm 0.17$ & $1.03 \pm 0.17$ & $1.10 \pm 0.17$ & 1.2 & K0 (G5..K3) & $0.59(0.19 . .1 .17)$ \\
\hline $\mathrm{J} 3422840+2536573$ & $18.48 \pm 0.27$ & $17.85 \pm 0.17$ & $0.44 \pm 0.32$ & $1.17 \pm 0.21$ & $2.03 \pm 0.21$ & $2.10 \pm 0.25$ & 8.9 & M0 (K4...M2) & $0.75(0.14 . .1 .44)$ \\
\hline $\mathrm{J} 3422845+2546246$ & $18.55 \pm 0.27$ & $17.73 \pm 0.17$ & $0.59 \pm 0.32$ & $0.91 \pm 0.22$ & $1.68 \pm 0.24$ & $2.31 \pm 0.22$ & 9.2 & M0 (K4..M1) & $0.88(0.34 . .1 .49)$ \\
\hline J3422864+2521384 & $18.06 \pm 0.26$ & $16.83 \pm 0.17$ & $1.24 \pm 0.32$ & $1.00 \pm 0.18$ & $1.50 \pm 0.20$ & $2.07 \pm 0.20$ & 4.3 & M2 (K5...M2) & $0.00(0.00 . .1 .26)$ \\
\hline J3422884+2526309 & $16.78 \pm 0.26$ & $16.23 \pm 0.17$ & $0.36 \pm 0.31$ & $0.64 \pm 0.18$ & $1.04 \pm 0.18$ & $1.10 \pm 0.20$ & 1.2 & K0 (G5..K3) & $0.72(0.04 . .1 .30)$ \\
\hline $\mathrm{J} 3422890+2543203$ & $17.85 \pm 0.26$ & $17.47 \pm 0.17$ & $0.28 \pm 0.31$ & $0.81 \pm 0.21$ & $1.62 \pm 0.22$ & $1.63 \pm$ & 3.2 & K4 (K0..K6) & $0.39(0.18 . .1 .44)$ \\
\hline $\mathrm{J} 3422906+2546379$ & $16.85 \pm 0.26$ & $15.95 \pm 0.17$ & $0.73 \pm 0.31$ & $0.69 \pm 0.18$ & $1.48 \pm 0.18$ & $1.61 \pm 0.18$ & 1.5 & K7 (K2..M0) & $0.66(0.05 . .1 .42)$ \\
\hline $\mathrm{J} 3422912+2539540$ & $17.06 \pm 0.26$ & $16.71 \pm 0.17$ & $0.36 \pm 0.31$ & $1.17 \pm 0.18$ & $1.31 \pm 0.20$ & $1.43 \pm 0.23$ & 7.1 & K4 (G3..K5) & $0.00(0.00 . .1 .48)$ \\
\hline J3422918+2535530 & $18.47 \pm 0.27$ & $18.38 \pm 0.19$ & $-0.35 \pm 0.32$ & $1.36 \pm 0.23$ & $1.98 \pm 0.25$ & $1.28 \pm$ & 21.3 & K4 (K1..M1) & $1.68(0.26 . .1 .68)$ \\
\hline J3422927+2546452 & $16.75 \pm 0.26$ & $15.92 \pm 0.17$ & $0.52 \pm 0.31$ & $-0.15 \pm 0.18$ & $0.10 \pm 0.20$ & $0.18 \pm 0.23$ & 5.5 & F0 (A2..G4) & $1.16(0.24 . .1 .47)$ \\
\hline J3422967+2545429 & $17.64 \pm 0.26$ & $16.98 \pm 0.17$ & $0.47 \pm 0.31$ & $0.74 \pm 0.19$ & $1.27 \pm 0.22$ & $1.34 \pm 0.24$ & 1.0 & K3 (K0..K7) & $0.70(0.06 . .1 .37)$ \\
\hline $\mathrm{J} 3422982+2530484$ & $17.95 \pm 0.26$ & $17.46 \pm 0.17$ & $0.46 \pm 0.32$ & $0.82 \pm 0.22$ & $1.18 \pm 0.29$ & $1.13 \pm$ & 1.5 & K3 (G0..K3) & $0.11(0.11 . .1 .33)$ \\
\hline J3422987+2540377 & $18.18 \pm 0.26$ & $17.10 \pm 0.17$ & $0.53 \pm 0.31$ & $1.26 \pm 0.18$ & $1.89 \pm 0.18$ & $1.96 \pm 0.19$ & 7.5 & M0 (M0..M3) & $2.05(0.62 . .2 .05)$ \\
\hline $\mathrm{J} 3422989+2523142$ & $19.88 \pm 0.31$ & $18.94 \pm 0.21$ & $0.33 \pm 0.38$ & $1.37 \pm 0.29$ & $1.92 \pm 0.33$ & $2.30 \pm 0.32$ & 8.3 & & ..2.29) \\
\hline J3422993+2528088 & $19.43 \pm 0.28$ & $19.08 \pm 0.21$ & $-0.20 \pm 0.36$ & $1.95 \pm 0.26$ & $2.52 \pm$ & $1.58 \pm$ & 24.5 & M0 (K2..M5) & $2.07(0.19 . .2 .07)$ \\
\hline $\mathrm{J} 3423036+2534270$ & $18.03 \pm 0.26$ & $17.56 \pm 0.17$ & $0.11 \pm 0.32$ & $0.91 \pm 0.21$ & $1.62 \pm 0.21$ & $1.79 \pm 0.23$ & 7.1 & K4 (K3..M1) & $1.34(0.25 . .1 .34)$ \\
\hline $\mathrm{J} 3423057+2542306$ & $13.74 \pm 0.26$ & $13.31 \pm 0.17$ & $0.32 \pm 0.31$ & $0.80 \pm 0.17$ & $1.06 \pm 0.17$ & $1.03 \pm 0.17$ & 3.7 & K0 (F7..K2) & $0.41(0.32 . .1 .44)$ \\
\hline $\mathrm{J} 3423060+2551397$ & $15.57 \pm 0.26$ & $14.90 \pm 0.17$ & $0.54 \pm 0.31$ & $0.91 \pm 0.17$ & $1.36 \pm 0.17$ & $1.38 \pm 0.17$ & 2.1 & K4 (K1..K6) & $0.50(0.06 . .1 .50)$ \\
\hline $\mathrm{J} 3423061+2521541$ & $19.22 \pm 0.28$ & $17.80 \pm 0.18$ & $1.28 \pm 0.33$ & $1.34 \pm 0.20$ & $1.86 \pm 0.24$ & $2.35 \pm 0.24$ & 2.0 & M3 (M1..M4) & $0.52(0.02 . .1 .48)$ \\
\hline $\mathrm{J} 3423079+2541327$ & $18.10 \pm 0.26$ & $17.58 \pm 0.17$ & $0.35 \pm 0.32$ & $0.93 \pm 0.22$ & $1.44 \pm 0.24$ & $1.59 \pm 0.30$ & 1.9 & K4 (K0..K5) & $0.66(0.37 . .1 .46)$ \\
\hline $\mathrm{J} 3423096+2535598$ & $18.68 \pm 0.27$ & $18.79 \pm 0.20$ & $-0.41 \pm 0.34$ & $1.87 \pm 0.25$ & $2.48 \pm 0.26$ & $2.74 \pm 0.29$ & 53.2 & M0 (K0...M4) & $1.12(0.16 . .1 .50)$ \\
\hline $\mathrm{J} 3423099+2542008$ & $15.06 \pm 0.26$ & $14.22 \pm 0.17$ & $0.57 \pm 0.31$ & $1.29 \pm 0.17$ & $1.87 \pm 0.17$ & $1.90 \pm 0.17$ & 7.6 & M0 (K6..M2) & $0.99(0.42 . .1 .40)$ \\
\hline $\mathrm{J} 3423137+2550369$ & $19.19 \pm 0.28$ & $17.80 \pm 0.17$ & $1.12 \pm 0.33$ & $1.17 \pm 0.21$ & $1.94 \pm 0.21$ & $1.78=$ & 2.9 & M2 (N & $6 . .1 .49)$ \\
\hline J3423146+2540166 & $14.55 \pm 0.26$ & $14.06 \pm 0.17$ & $0.41 \pm 0.31$ & $1.03 \pm 0.17$ & $1.39 \pm 0.17$ & $1.44 \pm 0.17$ & 3.5 & K4 (K0..K5) & $0.32(0.20 . .1 .44)$ \\
\hline $\mathrm{J} 3423147+2534231$ & $16.98 \pm 0.26$ & $16.25 \pm 0.17$ & $0.43 \pm 0.31$ & $0.86 \pm 0.18$ & 0.18 & 18 & 1.3 & K4 & $36)$ \\
\hline $\mathrm{J} 3423151+2547052$ & $19.01 \pm 0.27$ & $17.25 \pm 0.17$ & $1.75 \pm 0.32$ & $1.39 \pm 0.19$ & $2.07 \pm 0.20$ & & 1.9 & M4 & $0.03(0$ \\
\hline $\mathrm{J} 3423165+2522207$ & $16.12 \pm 0.26$ & $15.15 \pm 0.17$ & $0.88 \pm 0.31$ & $1.08 \pm 0.17$ & $1.70=$ & $1.79=$ & 1.6 & M0 & 39) \\
\hline $\mathrm{J} 3423172+2546072$ & $13.12 \pm 0.26$ & $12.53 \pm 0.17$ & $0.47 \pm 0.31$ & $0.98 \pm 0.17$ & $1.34 \pm 0.17$ & $1.44 \pm 0.17$ & 2. & & $0.44(0$ \\
\hline $\mathrm{J} 3423189+2528219$ & $16.37 \pm 0.26$ & 15.73 & $0.34 \pm 0.31$ & $0.75 \pm$ & 0.98 & 1.11 & 2.4 & K0 & 1.13 \\
\hline $\mathrm{J} 3423200+2521542$ & $18.97 \pm 0.27$ & $18.17 \pm 0.18$ & $0.34 \pm 0.33$ & $1.59 \pm 0.21$ & $1.82=$ & $2.15 \pm 0.24$ & 15.7 & M0 & $1.75(\mathrm{C})$ \\
\hline $\mathrm{J} 3423218+2552186$ & $16.57 \pm 0.26$ & $15.74 \pm 0.17$ & $0.52 \pm 0.31$ & $0.86 \pm 0.17$ & $1.39 \pm 0.18$ & 1.41 & 1.4 & $\mathrm{~K} 4$ & 1.20 \\
\hline $\mathrm{J} 3423224+2525436$ & $14.09 \pm 0.26$ & $13.48 \pm 0.17$ & $0.41 \pm 0.31$ & $0.73 \pm 0.17$ & $1.00 \pm 0.17$ & $1.02 \pm 0.17$ & 2. & K0 (F9..K2) & $0.75(0.53 . .1 .46)$ \\
\hline $\mathrm{J} 3423234+2544445$ & $17.03 \pm 0.26$ & $16.38 \pm 0.17$ & $0.27 \pm 0.31$ & $0.38 \pm 0.18$ & $0.55 \pm 0.21$ & $0.44 \pm 0.24$ & 1.8 & F5 (F4.. & $1.42(0.39 . .1 .42)$ \\
\hline $\mathrm{J} 3423236+2548440$ & $19.98 \pm 0.34$ & $18.14 \pm 0.18$ & $1.83 \pm 0.38$ & $1.82 \pm 0.21$ & $2.58 \pm 0.21$ & $2.59 \pm 0.26$ & 2.4 & M5 & $0.07(0$ \\
\hline $\mathrm{J} 3423243+2525129$ & $18.08 \pm 0.26$ & $17.50 \pm 0.17$ & $0.50 \pm 0.32$ & $0.84 \pm 0.22$ & $1.34 \pm 0.26$ & $1.75 \pm$ & 1.1 & (...K6) & $5.1 .43)$ \\
\hline $\mathrm{J} 3423257+2520442$ & $14.23 \pm 0.26$ & $13.56 \pm 0.17$ & $0.31 \pm 0.31$ & $0.48 \pm 0.17$ & $0.62 \pm 0.17$ & $0.65 \pm 0.17$ & 1.8 & F7 (F5..K2) & $1.36(0.24 . .1 .49)$ \\
\hline $\mathrm{J} 3423266+2535245$ & $16.54 \pm 0.26$ & $15.94 \pm 0.17$ & $0.33 \pm 0.31$ & $0.79 \pm 0.18$ & $0.97 \pm 0.18$ & $1.10 \pm 0.18$ & 3.1 & K0 (G5..K3) & $0.99(0.25 . .1 .48)$ \\
\hline $\mathrm{J} 3423268+2528088$ & $17.92 \pm 0.26$ & $17.12 \pm 0.17$ & $0.41 \pm 0.31$ & $0.68 \pm 0.20$ & $1.05 \pm 0.21$ & $1.15 \pm 0.24$ & 1.3 & K1 (K0..K7) & $1.48(0.33 . .1 .48)$ \\
\hline $\mathrm{J} 3423274+2548595$ & $17.48 \pm 0.26$ & $17.32 \pm 0.17$ & $0.16 \pm 0.31$ & $0.47 \pm 0.24$ & $0.88 \pm 0.30$ & $1.62 \pm 0.28$ & 8.4 & K0 (F5..K0) & $0.00(0.00 . .1 .14)$ \\
\hline $\mathrm{J} 3423275+2536502$ & $18.44 \pm 0.27$ & $18.16 \pm 0.18$ & $-0.14 \pm 0.32$ & $1.33 \pm 0.22$ & $1.47 \pm$ & $1.22 \pm$ & 11.1 & K4 (K1..M1) & $1.59(0.04 . .1 .59)$ \\
\hline $\mathrm{J} 3423281+2520581$ & $17.24 \pm 0.26$ & $17.83 \pm 0.18$ & $-0.60 \pm 0.32$ & $1.10 \pm 0.24$ & 33 & & 21.4 & & \\
\hline $\mathrm{J} 3423307+2528358$ & $18.97 \pm 0.27$ & $17.55 \pm 0.17$ & $1.19 \pm 0.32$ & $1.42 \pm 0.19$ & $1.96 \pm 0.20$ & $2.33 \pm 0.21$ & 2.6 & M3 (M1..M4) & $0.86(0.10 . .1 .50)$ \\
\hline $\mathrm{J} 3423309+2551145$ & $18.10 \pm 0.26$ & $17.21 \pm 0.17$ & $0.64 \pm 0.32$ & $0.64 \pm 0.21$ & & & 5. & & \\
\hline+2542441 & 18.31 & $17.36=$ & $0.81 \pm 0.32$ & $1.13 \pm 0.19$ & 0.20 & $1.74 \pm 0.25$ & 2.3 & M0 & 0.50 \\
\hline $\mathrm{J} 3423345+2535390$ & & & \pm 0.31 & & & & 2.8 & & \\
\hline $\mathrm{J} 3423347+2522153$ & $15.60 \pm 0.26$ & $15.19 \pm 0.17$ & $0.36 \pm 0.31$ & $-0.95 \pm 0.20$ & $-0.86 \pm 0.25$ & $-0.75 \pm 0.31$ & 14.0 & B0 (B0..B5) & $0.19(0.03 . .1 .04)$ \\
\hline $\mathrm{J} 3423359+2549070$ & $15.60 \pm 0.26$ & $14.97 \pm 0.17$ & $0.50 \pm 0.31$ & & & & 20 & & \\
\hline $\mathrm{J} 3423364+2532233$ & $15.93 \pm 0.26$ & $15.47 \pm 0.17$ & $0.39 \pm 0.31$ & $1.00 \pm 0.17$ & $1.41 \pm 0.17$ & $1.46 \pm 0.18$ & 3.0 & K4 (K0..K5) & $0.26(0.14 . .1 .31)$ \\
\hline $\mathrm{J} 3423375+2520031$ & $18.04 \pm 0.27$ & $17.30 \pm 0.17$ & $0.36 \pm 0.32$ & $0.40 \pm 0.22$ & & & 1.0 & G4 (F9...K7) & $1.40(0.11 . .1 .40)$ \\
\hline $\mathrm{J} 3423380+2527003$ & $18.06 \pm 0.26$ & $17.14 \pm 0.17$ & $0.89 \pm 0.31$ & $0.46 \pm 0.23$ & $1.36 \pm 0.24$ & $1.65 \pm 0.25$ & 4.2 & K7 (K1..K7) & $0.14(0.14 . .1 .12)$ \\
\hline $\mathrm{J} 3423438+2522110$ & $15.06 \pm 0.26$ & $14.40 \pm 0.17$ & $0.28 \pm 0.31$ & $0.49 \pm 0.17$ & \pm 0.17 & 7 & 2.1 & F7 (F6..K2) & $2.1 .43)$ \\
\hline $\mathrm{J} 3423443+2535271$ & $18.28 \pm 0.26$ & $16.76 \pm 0.17$ & $1.24 \pm 0.31$ & $1.37 \pm 0.18$ & $2.08 \pm 0.18$ & $2.17 \pm 0.18$ & 2.3 & M3 (M3..M4) & $1.02(0.01 . .1 .31)$ \\
\hline $\mathrm{J} 3423448+2531234$ & $15.71 \pm 0.26$ & $15.07 \pm 0.17$ & $0.49 \pm 0.31$ & $0.99 \pm 0.17$ & $=0.17$ & & 3. & K4 (K0..K6) & $0.58(0.13 . .1 .50)$ \\
\hline $\mathrm{J} 3423454+2544103$ & $18.19 \pm 0.26$ & $17.35 \pm 0.17$ & $0.54 \pm 0.32$ & $0.76 \pm 0.21$ & $1.56 \pm 0.21$ & $1.17 \pm 0.29$ & 4.0 & K4 (K3...M0) & $1.15(0.35 .1 .44)$ \\
\hline $\mathrm{J} 3423533+2536209$ & $16.56 \pm 0.26$ & $15.93 \pm 0.17$ & $0.38 \pm 0.31$ & $0.98 \pm 0.17$ & $1.39 \pm 0.18$ & $1.51 \pm 0.18$ & 2.5 & K4 (K3..K6) & $0.92(0.64 . .1 .43)$ \\
\hline+2552392 & $18.70 \pm 0.27$ & $17.82 \exists$ & $0.46 \pm 0.32$ & $0.81 \pm 0.24$ & $0.73 \pm 0.36$ & 0.32 & 3.8 & .M0) & $1.57(0.04 . .1 .57)$ \\
\hline+2549546 & $17.92 \pm 0.26$ & $17.04=$ & $0.47=$ & & & $101+0$ & 1.8 & ..K7) & $1.52(0.01 . .1 .52)$ \\
\hline+2552179 & $17.35 \pm 0.26$ & $16.81=$ & $0.37 \pm 0.31$ & $0.70=$ & 0.87 & $1.18=$ & 2.4 & K0 & $7.1 .47)$ \\
\hline $\mathrm{J} 342$ & & & & & & & 2. & & $2.1 .44)$ \\
\hline $\begin{array}{r}+2524233 \\
\end{array}$ & 18 & & 31 & & & & 1. & 12) & .48) \\
\hline $\mathrm{J} 3423608+2552535$ & $19.17 \pm 0.27$ & & $0.89 \pm 0.32$ & 0.5 & & 1.76 & 5.2 & 6...M3) & $1 . .1 .53)$ \\
\hline+2526561 & & & & & & & 3.6 & & 45) \\
\hline $\mathrm{J} 3423628+2541187$ & $17.87 \pm 0.26$ & & $-0.02 \pm 0$ & 1. & 1.6 & $1.79 \pm 0.20$ & 12.1 & K4 & $1.88(0$ \\
\hline J342363 & 20.2 & 18 & $1.69 \pm$ & & & & 1.1 & M5) & 0.86 \\
\hline $\mathrm{J} 3423651+2543038$ & & & $0.32 \pm 0.31$ & \pm 0.17 & 1. & 0.17 & 4.2 & K4 & $1.24(0$ \\
\hline J34236: & 18.76 & & -0. & & & & 35.6 & 2) & 97) \\
\hline $\mathrm{J} 3423686+2548163$ & $17.67 \pm 0.26$ & $17.33 \pm 0.17$ & $0.33 \pm 0.31$ & \pm 0.22 & $1.52 \pm 0.23$ & 28 & 3.3 & K4 (K0..K5) & $0.05(0.01 . .0 .90)$ \\
\hline J3423693+2541344 & & & & & & & 5.9 & & \\
\hline $\mathrm{J} 3423697+2524276$ & $17.37 \pm 0.26$ & $16.50 \pm 0.17$ & $0.52 \pm 0.31$ & $0.60 \pm 0.18$ & $1.00 \pm 0.19$ & $1.10 \pm 0.20$ & 1.5 & K1 (G8..K7) & $1.29(0.26 . .1 .46)$ \\
\hline $\mathrm{J} 3423709+2549015$ & $17.33 \pm 0.26$ & $16.42 \pm 0.17$ & $0.59 \pm 0.31$ & $0.76 \pm 0.18$ & $1.21 \pm 0.19$ & & 3.8 & K5 (K3..M1) & $1.20(0.33 . .1 .35)$ \\
\hline $\mathrm{J} 3423716+2524461$ & $14.74 \pm 0.26$ & $14.03 \pm 0.17$ & $0.41 \pm 0.31$ & & $1.00 \pm 0.17$ & \pm 0.17 & 2.2 & K0 (G5..K6) & $1.10(0.24 . .1 .49)$ \\
\hline 2544518 & & & & & & & 1.1 & G7 (F7..K0) & 75..1.49) \\
\hline $\mathrm{J} 3423730+2551192$ & $19.71 \pm 0.30$ & $18.01 \pm 0.18$ & $1.49 \pm 0.35$ & $0.72 \pm 0.30$ & $1.23 \pm 0.33$ & $2.07 \pm 0.28$ & 7.9 & 6.M3) & $7.1 .35)$ \\
\hline & & & & & & & 12. & & \\
\hline $\mathrm{J} 3423744+2531523$ & $17.60 \pm 0.26$ & $16.48 \pm 0.17$ & $0.84 \pm 0.31$ & $1.10 \pm 0.18$ & $1.71 \pm 0.18$ & $1.83 \pm 0.18$ & 1.6 & M0 (M0..M2) & $3.1 .15)$ \\
\hline $\mathrm{J} 3423750+2520194$ & $15.10 \pm 0.26$ & & $0.40 \pm 0.31$ & $0.58 \pm 0$ & & & 1.8 & $\mathrm{G} 7(\mathrm{G} 0 . . \mathrm{K} 2)$ & $1.24(0.67 . .1 .48)$ \\
\hline $\mathrm{J} 3423767+2527355$ & $18.16 \pm 0.26$ & $17.40 \pm 0.17$ & $0.39 \pm 0.32$ & $0.49 \pm 0.22$ & $0.84 \pm 0.28$ & & 1.0 & G7 (G4..K3) & $1.36(0.53 . .1 .44)$ \\
\hline $\mathrm{J} 34$ & 15. & & 0. & $0.55 \pm 0$ & $0.86 \pm 0$ & & 2.1 & G7 (G3..K3) & $1.40(0.48 . .1 .47)$ \\
\hline J3423771+2548399 & $15.89 \pm 0.26$ & $15.28 \pm 0.17$ & $0.10 \pm 0.31$ & $-0.89 \pm 0.19$ & $-0.77 \pm 0.20$ & $-0.50 \pm 0.21$ & 11.8 & B0 (B0..A3) & $1.94(0.06 . .1 .94)$ \\
\hline $\mathrm{J} 3423778+2532034$ & $17.15 \pm 0.26$ & $16.51 \pm 0.17$ & $0.53 \pm 0.31$ & $0.80 \pm 0.18$ & $1.37 \pm 0.19$ & $1.44 \pm 0.20$ & 1.1 & K4 (K0..K6) & $0.43(0.30 . .1 .41)$ \\
\hline
\end{tabular}




\begin{tabular}{|c|c|c|c|c|c|c|c|c|c|}
\hline \multirow[b]{2}{*}{ 2MASS-ID } & \multicolumn{2}{|c|}{ measured magnitudes } & & extinction co & ected colors & & & & \\
\hline & Rmag & Imag & R-I & I-J & $\mathrm{I}-\mathrm{H}$ & $\mathrm{I}-\mathrm{K}_{s}$ & $\chi^{2}$ & SpT & $\mathrm{Av}$ \\
\hline $\mathrm{J} 3423791+2535418$ & $17.22 \pm 0.26$ & $16.56 \pm 0.17$ & $0.26 \pm 0.31$ & $0.60 \pm 0.18$ & $0.63 \pm 0.21$ & $0.56 \pm 0.25$ & 3.9 & F7 (F6..K2) & $1.50(0.14 . .1 .50)$ \\
\hline $\mathrm{J} 3423793+2546135$ & $18.04 \pm 0.26$ & $17.24 \pm 0.17$ & $0.63 \pm 0.32$ & $0.82 \pm 0.20$ & $1.51 \pm 0.21$ & $1.24 \pm 0.29$ & 3.0 & K5 (K2..K7) & $0.64(0.01 . .1 .38)$ \\
\hline $\mathrm{J} 3423796+2522018$ & $19.47 \pm 0.29$ & $18.08 \pm 0.18$ & $1.32 \pm 0.34$ & $1.32 \pm 0.23$ & $1.92 \pm 0.26$ & $2.27 \pm 0.28$ & 1.2 & M3 (M1..M3) & $0.28(0.28 . .1 .47)$ \\
\hline $\mathrm{J} 3423798+2533514$ & $16.92 \pm 0.26$ & $16.14 \pm 0.17$ & $0.46 \pm 0.31$ & $0.18 \pm 0.20$ & $0.43 \pm 0.23$ & $1.15 \pm 0.20$ & 9.7 & G6 (F6..K4) & $1.20(0.39 . .1 .48)$ \\
\hline $\mathrm{J} 3423807+2542259$ & $17.72 \pm 0.26$ & $17.15 \pm 0.17$ & $0.32 \pm 0.31$ & $0.77 \pm 0.20$ & $1.36 \pm 0.21$ & $1.79 \pm 0.21$ & 4.6 & $\mathrm{~K} 4(\mathrm{~K} 2 . . \mathrm{K} 7)$ & $0.95(0.20 . .1 .38)$ \\
\hline $\mathrm{J} 3423823+2526207$ & $17.28 \pm 0.26$ & $16.20 \pm 0.17$ & $1.02 \pm 0.31$ & $1.04 \pm 0.17$ & $1.81 \pm 0.18$ & $1.82 \pm 0.19$ & 1.8 & M1 (K5..M1) & $0.23(0.23 . .1 .47)$ \\
\hline $\mathrm{J} 3423832+2548448$ & $15.76 \pm 0.26$ & $15.07 \pm 0.17$ & $0.50 \pm 0.31$ & $0.78 \pm 0.17$ & $1.26 \pm 0.17$ & $1.29 \pm 0.17$ & 1.3 & K3 (K0..K7) & $0.73(0.14 . .1 .15)$ \\
\hline $\mathrm{J} 3423886+2538403$ & $18.78 \pm 0.27$ & $18.15 \pm 0.18$ & $0.28 \pm 0.32$ & $1.48 \pm 0.21$ & $2.05 \pm 0.23$ & $2.10 \pm 0.27$ & 15.6 & M0 (K4..M3) & $1.32(0.40 . .1 .49)$ \\
\hline $\mathrm{J} 3423887+2519428$ & $17.37 \pm 0.26$ & $16.40 \pm 0.17$ & $0.84 \pm 0.31$ & $1.12 \pm 0.18$ & $1.66 \pm 0.18$ & $1.84 \pm 0.19$ & 1.8 & M0 (K5..M1) & $0.46(0.02 . .1 .40)$ \\
\hline $\mathrm{J} 3423897+2526592$ & $16.90 \pm 0.26$ & $15.38 \pm 0.17$ & $1.27 \pm 0.31$ & $-0.88 \pm 0.19$ & $-0.32 \pm 0.20$ & $-0.01 \pm 0.21$ & 59.5 & F0 (B1..K0) & $0.94(0.12 . .1 .43)$ \\
\hline $\mathrm{J} 3423910+2536322$ & $18.57 \pm 0.27$ & $18.47 \pm 0.19$ & $-0.44 \pm 0.32$ & $1.52 \pm 0.23$ & $2.07 \pm 0.23$ & $1.80 \pm 0.34$ & 33.0 & K4 (K1..M3) & $2.02(0.26 . .2 .02)$ \\
\hline $\mathrm{J} 3423935+2520243$ & $18.53 \pm 0.27$ & $18.72 \pm 0.20$ & $-0.71 \pm 0.34$ & $1.82 \pm 0.24$ & $2.15 \pm 0.26$ & $2.09 \pm 0.32$ & 49.9 & K4 (G9...M3) & $1.98(0.18 . .1 .98)$ \\
\hline $\mathrm{J} 3423948+2529448$ & $14.78 \pm 0.26$ & $14.29 \pm 0.17$ & $0.38 \pm 0.31$ & $0.78 \pm 0.17$ & $1.01 \pm 0.17$ & $1.01 \pm 0.17$ & 3.4 & K0 (F5..K2) & $0.41(0.36 . .1 .42)$ \\
\hline $\mathrm{J} 3423950+2548426$ & $15.53 \pm 0.26$ & $14.99 \pm 0.17$ & $0.34 \pm 0.31$ & $0.70 \pm 0.17$ & $0.87 \pm 0.17$ & $0.84 \pm 0.18$ & 3.5 & G7 (F6..K2) & $0.75(0.25 . .1 .42)$ \\
\hline $\mathrm{J} 3423964+2549449$ & $17.29 \pm 0.26$ & $16.53 \pm 0.17$ & $0.43 \pm 0.31$ & $0.88 \pm 0.18$ & $1.36 \pm 0.18$ & $1.54 \pm 0.19$ & 1.5 & K4 (K3..M0) & $1.24(0.06 . .1 .46)$ \\
\hline $\mathrm{J} 3423972+2524127$ & $15.78 \pm 0.26$ & $15.17 \pm 0.17$ & $0.27 \pm 0.31$ & $0.51 \pm 0.17$ & $0.64 \pm 0.18$ & $0.66 \pm 0.18$ & 2.1 & F7 (F5..K0) & $1.29(0.21 . .1 .40)$ \\
\hline $\mathrm{J} 3423985+2520363$ & $16.84 \pm 0.26$ & $16.15 \pm 0.17$ & $0.41 \pm 0.31$ & $0.62 \pm 0.18$ & $0.93 \pm 0.19$ & $1.13 \pm 0.19$ & 1.5 & K0 (G5..K5) & $1.03(0.03 . .1 .48)$ \\
\hline $\mathrm{J} 3423986+2551550$ & $17.34 \pm 0.26$ & $16.35 \pm 0.17$ & $0.91 \pm 0.31$ & $0.51 \pm 0.19$ & $1.14 \pm 0.20$ & $1.77 \pm 0.20$ & 8.2 & K7 (G9..K7) & $0.30(0.25 . .1 .36)$ \\
\hline $\mathrm{J} 3423996+2549260$ & $20.36 \pm 0.37$ & $18.44 \pm 0.18$ & $1.60 \pm 0.41$ & $2.04 \pm 0.21$ & $2.30 \pm 0.23$ & $2.95 \pm 0.22$ & 5.4 & M5 (M5..M6.5) & $1.21(0.04 . .1 .43)$ \\
\hline $\mathrm{J} 3424027+2531571$ & $22.98 \pm 2.42$ & $18.09 \pm 0.18$ & $4.89 \pm 2.43$ & $1.58 \pm 0.22$ & $2.04 \pm 0.27$ & $2.12 \pm 0.33$ & 8.8 & M4 (K7..M4) & $0.00(0.00 . .1 .42)$ \\
\hline $\mathrm{J} 3424030+2554103$ & $19.52 \pm 0.26$ & $16.44 \pm 0.17$ & $3.09 \pm 0.31$ & $0.69 \pm 0.18$ & $1.01 \pm 0.20$ & $1.48 \pm 0.22$ & 103.1 & M4 (K4..M6) & $0.00(0.00 . .1 .49)$ \\
\hline $\mathrm{J} 3424050+2528426$ & $16.67 \pm 0.26$ & $15.96 \pm 0.17$ & $0.49 \pm 0.31$ & $0.85 \pm 0.17$ & $1.24 \pm 0.18$ & $1.26 \pm 0.18$ & 2.2 & $\mathrm{~K} 3$ (K1..K7) & $0.83(0.06 . .1 .12)$ \\
\hline $\mathrm{J} 3424061+2526370$ & $14.98 \pm 0.26$ & $14.36 \pm 0.17$ & $0.37 \pm 0.31$ & $0.75 \pm 0.17$ & $1.03 \pm 0.17$ & $1.03 \pm 0.17$ & 2.7 & K0 (G7..K5) & $0.91(0.03 . .1 .19)$ \\
\hline $\mathrm{J} 3424097+2553279$ & $16.86 \pm 0.26$ & $15.70 \pm 0.17$ & $0.86 \pm 0.31$ & $1.08 \pm 0.17$ & $1.70 \pm 0.17$ & $1.82 \pm 0.17$ & 1.4 & M0 (M0..M2) & $1.15(0.57 . .1 .15)$ \\
\hline $\mathrm{J} 3424099+2542574$ & $16.30 \pm 0.26$ & $15.34 \pm 0.17$ & $0.69 \pm 0.31$ & $1.20 \pm 0.17$ & $1.75 \pm 0.17$ & $1.90 \pm 0.17$ & 3.7 & M0 (M0..M2) & $1.02(0.44 . .1 .02)$ \\
\hline $\mathrm{J} 3424129+2528094$ & $17.96 \pm 0.26$ & $17.09 \pm 0.17$ & $0.51 \pm 0.31$ & $0.88 \pm 0.19$ & $1.46 \pm 0.19$ & $1.31 \pm 0.22$ & 2.5 & $\mathrm{~K} 4(\mathrm{~K} 4 . . \mathrm{K} 7)$ & $1.39(0.74 . .1 .47)$ \\
\hline $\mathrm{J} 3424133+2541214$ & $17.08 \pm 0.26$ & $16.37 \pm 0.17$ & $0.52 \pm 0.31$ & $0.86 \pm 0.18$ & $1.33 \pm 0.18$ & $1.45 \pm 0.19$ & 1.3 & $\mathrm{~K} 4(\mathrm{~K} 1 . . \mathrm{K} 7)$ & $0.70(0.30 . .1 .27)$ \\
\hline $\mathrm{J} 3424138+2536581$ & $16.48 \pm 0.26$ & $15.96 \pm 0.17$ & $0.48 \pm 0.31$ & $0.95 \pm 0.18$ & $1.20 \pm 0.18$ & $1.24 \pm 0.19$ & 4.1 & K3 (F6..K5) & $0.11(0.05 . .1 .47)$ \\
\hline $\mathrm{J} 3424145+2549074$ & $18.83 \pm 0.27$ & $17.88 \pm 0.18$ & $0.60 \pm 0.32$ & $1.28 \pm 0.21$ & $1.67 \pm 0.26$ & $2.07 \pm 0.24$ & 5.4 & M0 (M0..M3) & $1.31(0.37 . .1 .31)$ \\
\hline $\mathrm{J} 3424148+2539468$ & $14.83 \pm 0.26$ & $14.33 \pm 0.17$ & $0.50 \pm 0.31$ & $0.97 \pm 0.17$ & $1.34 \pm 0.17$ & $1.36 \pm 0.17$ & 3.3 & K4 (G6..K5) & $0.00(0.00 . .1 .26)$ \\
\hline $\mathrm{J} 3424161+2538411$ & $16.37 \pm 0.26$ & $15.62 \pm 0.17$ & $0.54 \pm 0.31$ & $1.19 \pm 0.17$ & $1.86 \pm 0.17$ & $2.01 \pm 0.17$ & 6.9 & M0 (K7..M2) & $0.79(0.14 . .1 .11)$ \\
\hline $\mathrm{J} 3424166+2551150$ & $15.86 \pm 0.26$ & $14.92 \pm 0.17$ & $0.76 \pm 0.31$ & $1.12 \pm 0.17$ & $1.76 \pm 0.17$ & $1.85 \pm 0.17$ & 2.2 & M0 (K5..M1) & $0.67(0.49 . .1 .43)$ \\
\hline $\mathrm{J} 3424167+2544234$ & $16.34 \pm 0.26$ & $15.49 \pm 0.17$ & $0.68 \pm 0.31$ & $1.15 \pm 0.17$ & $1.77 \pm 0.17$ & $1.93 \pm 0.17$ & 3.2 & M0 (K4..M2) & $0.63(0.13 . .1 .49)$ \\
\hline $\mathrm{J} 3424182+2533069$ & $17.90 \pm 0.26$ & $17.08 \pm 0.17$ & $0.39 \pm 0.31$ & $1.39 \pm 0.18$ & $1.88 \pm 0.18$ & $2.07 \pm 0.19$ & 13.5 & M0 (M0..M2) & $1.59(0.40 . .1 .59)$ \\
\hline $\mathrm{J} 3424186+2530177$ & $17.21 \pm 0.26$ & $16.59 \pm 0.17$ & $0.36 \pm 0.31$ & $0.96 \pm 0.18$ & $1.42 \pm 0.18$ & $1.52 \pm 0.20$ & 2.3 & K4 (K3..K5) & $0.97(0.76 . .1 .49)$ \\
\hline $\mathrm{J} 3424187+2537361$ & $18.24 \pm 0.26$ & $17.74 \pm 0.17$ & $0.30 \pm 0.32$ & $0.90 \pm 0.24$ & $1.60 \pm \ldots$ & $1.66 \pm 0.30$ & 2.0 & $\mathrm{~K} 4$ (K2..K6) & $0.73(0.23 . .1 .08)$ \\
\hline $\mathrm{J} 3424199+2545339$ & $11.10 \pm 0.26$ & $10.70 \pm 0.17$ & $0.33 \pm 0.31$ & $0.69 \pm 0.17$ & $0.83 \pm 0.17$ & $0.89 \pm 0.17$ & 3.3 & G7 (F4..G8) & $0.22(0.22 . .1 .08)$ \\
\hline $\mathrm{J} 3424200+2535041$ & $15.31 \pm 0.26$ & $14.56 \pm 0.17$ & $0.30 \pm 0.31$ & $1.00 \pm 0.17$ & $1.48 \pm 0.17$ & $1.51 \pm 0.17$ & 3.6 & K4 (K4..M1) & $1.68(0.24 . .1 .68)$ \\
\hline $\mathrm{J} 3424206+2529069$ & $17.27 \pm 0.26$ & $16.55 \pm 0.17$ & $0.37 \pm 0.31$ & $0.54 \pm 0.18$ & $0.76 \pm 0.21$ & $1.01 \pm 0.22$ & 1.7 & G7 (G6..K6) & $1.33(0.04 . .1 .34)$ \\
\hline $\mathrm{J} 3424207+2541221$ & $18.36 \pm 0.27$ & $17.30 \pm 0.17$ & $0.58 \pm 0.32$ & $0.15 \pm 0.23$ & $0.30 \pm \ldots$ & $0.36 \pm \ldots$ & 2.1 & G0 (F4..K6) & $1.83(0.28 . .1 .87)$ \\
\hline $\mathrm{J} 3424209+2534098$ & $16.86 \pm 0.26$ & $15.74 \pm 0.17$ & $0.75 \pm 0.31$ & $1.19 \pm 0.17$ & $1.70 \pm 0.17$ & $1.88 \pm 0.17$ & 3.0 & M0 (M0..M3) & $1.37(0.06 . .1 .48)$ \\
\hline $\mathrm{J} 3424218+2530372$ & $17.52 \pm 0.26$ & $16.83 \pm 0.17$ & $0.33 \pm 0.31$ & $0.65 \pm 0.19$ & $0.93 \pm 0.21$ & $0.77 \pm 0.27$ & 2.8 & G7 (G5..K3) & $1.35(0.05 . .1 .45)$ \\
\hline $\mathrm{J} 3424229+2548150$ & $15.35 \pm 0.26$ & $14.80 \pm 0.17$ & $0.32 \pm 0.31$ & $0.66 \pm 0.17$ & $0.88 \pm 0.17$ & $0.89 \pm 0.17$ & 2.6 & G7 (G2..K3) & $0.90(0.13 . .1 .22)$ \\
\hline $\mathrm{J} 3424234+2552572$ & $16.18 \pm 0.26$ & $15.57 \pm 0.17$ & $0.35 \pm 0.31$ & $0.58 \pm 0.17$ & $0.85 \pm 0.18$ & $0.92 \pm 0.18$ & 1.5 & G7 (F6..G9) & $0.96(0.72 . .1 .48)$ \\
\hline $\mathrm{J} 3424266+2524015$ & $15.21 \pm 0.26$ & $14.38 \pm 0.17$ & $0.50 \pm 0.31$ & $0.89 \pm 0.17$ & $1.39 \pm 0.17$ & $1.41 \pm 0.17$ & 1.6 & K4 (K4..M0) & $1.23(0.02 . .1 .40)$ \\
\hline $\mathrm{J} 3424269+2527372$ & $22.12 \pm 1.20$ & $18.58 \pm 0.19$ & $3.33 \pm 1.22$ & $1.38 \pm 0.28$ & $2.86 \pm 0.22$ & $2.79 \pm 0.26$ & 9.7 & M6 (M4..M7) & $0.79(0.03 . .1 .47)$ \\
\hline $\mathrm{J} 3424270+2543241$ & $15.19 \pm 0.26$ & $14.39 \pm 0.17$ & $0.34 \pm 0.31$ & $0.99 \pm 0.17$ & $1.45 \pm 0.17$ & $1.50 \pm 0.17$ & 3.0 & K4 (K4..M0) & $1.73(0.69 . .1 .80)$ \\
\hline $\mathrm{J} 3424336+2528227$ & $20.82 \pm 0.43$ & $19.26 \pm 0.25$ & $1.27 \pm 0.50$ & $2.03 \pm 0.31$ & $2.72 \pm 0.33$ & $3.13 \pm 0.33$ & 7.0 & M5 (M4..M6.5) & $1.07(0.21 . .1 .39)$ \\
\hline $\mathrm{J} 3424338+2530099$ & $19.25 \pm 0.27$ & $18.13 \pm 0.18$ & $0.72 \pm 0.33$ & $1.18 \pm 0.24$ & $1.41 \pm 0.29$ & $2.41 \pm 0.23$ & 11.5 & M1 (M0..M4) & $1.47(0.03 . .1 .47)$ \\
\hline $\mathrm{J} 3424354+2533068$ & $15.67 \pm 0.26$ & $15.16 \pm 0.17$ & $0.40 \pm 0.31$ & $1.05 \pm 0.17$ & $1.39 \pm 0.17$ & $1.44 \pm 0.17$ & 4.1 & $\mathrm{~K} 4$ (K1..K7) & $0.42(0.06 . .1 .46)$ \\
\hline $\mathrm{J} 3424376+2522076$ & $18.02 \pm 0.26$ & $16.90 \pm 0.17$ & $1.11 \pm 0.32$ & $1.01 \pm 0.19$ & $1.69 \pm 0.19$ & $1.80 \pm 0.21$ & 1.5 & M1 (K5..M1) & $0.00(0.00 . .1 .43)$ \\
\hline $\mathrm{J} 3424384+2546445$ & $17.17 \pm 0.26$ & $16.50 \pm 0.17$ & $0.45 \pm 0.31$ & $0.96 \pm 0.18$ & $1.36 \pm 0.18$ & $1.46 \pm 0.20$ & 2.2 & K4 (K3..K6) & $0.83(0.45 . .1 .05)$ \\
\hline $\mathrm{J} 3424396+2529199$ & $16.01 \pm 0.26$ & $15.38 \pm 0.17$ & $0.34 \pm 0.31$ & $0.78 \pm 0.17$ & $0.99 \pm 0.18$ & $1.08 \pm 0.18$ & 3.0 & $\mathrm{~K} 0(\mathrm{G} 5 . . \mathrm{K} 7)$ & $1.09(0.01 . .1 .48)$ \\
\hline $\mathrm{J} 3424399+2540434$ & $18.44 \pm 0.27$ & $16.94 \pm 0.17$ & $1.18 \pm 0.32$ & $1.46 \pm 0.18$ & $2.11 \pm 0.18$ & $2.17 \pm 0.19$ & 3.6 & M3 (M3..M4) & $1.23(0.12 . .1 .49)$ \\
\hline $\mathrm{J} 3424418+2550572$ & $17.77 \pm 0.26$ & $17.18 \pm 0.17$ & $0.47 \pm 0.31$ & $0.65 \pm 0.21$ & $1.17 \pm 0.24$ & $1.79 \pm 0.22$ & 6.1 & K4 (G8..K6) & $0.47(0.24 . .1 .30)$ \\
\hline $\mathrm{J} 3424432+2522484$ & $16.47 \pm 0.26$ & $15.69 \pm 0.17$ & $0.42 \pm 0.31$ & $0.65 \pm 0.17$ & $1.02 \pm 0.18$ & $1.04 \pm 0.18$ & 1.5 & $\mathrm{~K} 0(\mathrm{~K} 0 . . \mathrm{K} 7)$ & $1.35(0.17 . .1 .35)$ \\
\hline $\mathrm{J} 3424454+2548071$ & $18.72 \pm 0.27$ & $17.40 \pm 0.17$ & $1.09 \pm 0.32$ & $0.53 \pm 0.23$ & $1.20 \pm 0.24$ & $1.41 \pm 0.28$ & 4.9 & $\mathrm{~K} 7$ (K2..M2) & $0.85(0.01 . .1 .47)$ \\
\hline $\mathrm{J} 3424481+2521196$ & $19.84 \pm 0.32$ & $18.01 \pm 0.18$ & $1.81 \pm 0.36$ & $1.47 \pm 0.22$ & $1.97 \pm 0.26$ & $2.30 \pm 0.27$ & 2.0 & M4 (M3..M4) & $0.06(0.06 . .1 .15)$ \\
\hline $\mathrm{J} 3424491+2552069$ & $13.85 \pm 0.26$ & $13.15 \pm 0.17$ & $0.38 \pm 0.31$ & $0.68 \pm 0.17$ & $1.01 \pm 0.17$ & $1.08 \pm 0.17$ & 1.5 & K0 (G9..K6) & $1.18(0.08 . .1 .33)$ \\
\hline $\mathrm{J} 3424504+2539502$ & $15.58 \pm 0.26$ & $14.95 \pm 0.17$ & $0.41 \pm 0.31$ & $0.99 \pm 0.17$ & $1.42 \pm 0.17$ & $1.44 \pm 0.17$ & 2.8 & $\mathrm{~K} 4$ (K1..K5) & $0.85(0.63 . .1 .42)$ \\
\hline $\mathrm{J} 3424520+2547478$ & $18.29 \pm 0.27$ & $17.53 \pm 0.17$ & $0.35 \pm 0.32$ & $1.06 \pm 0.20$ & $1.22 \pm 0.23$ & $1.65 \pm 0.23$ & 5.2 & K4 (K3..M2) & $1.53(0.08 . .1 .53)$ \\
\hline $\mathrm{J} 3424544+2537290$ & $15.88 \pm 0.26$ & $15.37 \pm 0.17$ & $0.51 \pm 0.31$ & $0.98 \pm 0.17$ & $1.33 \pm 0.18$ & $1.40 \pm 0.18$ & 3.0 & K4 (G6..K4) & $0.02(0.02 . .1 .40)$ \\
\hline $\mathrm{J} 3424549+2543591$ & $15.69 \pm 0.26$ & $15.10 \pm 0.17$ & $0.52 \pm 0.31$ & $0.92 \pm 0.17$ & $1.36 \pm 0.17$ & $1.41 \pm 0.17$ & 1.9 & $\mathrm{~K} 4(\mathrm{~K} 0 . . \mathrm{K} 7)$ & $0.29(0.03 . .1 .33)$ \\
\hline $\mathrm{J} 3424552+2533010$ & $18.30 \pm 0.26$ & $16.83 \pm 0.17$ & $1.46 \pm 0.32$ & $2.02 \pm 0.17$ & $2.65 \pm 0.18$ & $2.92 \pm 0.18$ & 10.0 & M5 (M3..M5) & $0.07(0.07 . .1 .33)$ \\
\hline $\mathrm{J} 3424597+2523295$ & $19.00 \pm 0.27$ & $18.15 \pm 0.18$ & $0.41 \pm 0.33$ & $1.39 \pm 0.22$ & $1.35 \pm$ & $2.05 \pm$ & 6.5 & M0 (K4..M4) & $1.66(0.05 . .1 .66)$ \\
\hline $\mathrm{J} 3424600+2539125$ & $17.35 \pm 0.26$ & $16.83 \pm 0.17$ & $0.43 \pm 0.31$ & $1.02 \pm 0.19$ & $1.31 \pm 0.20$ & $1.48 \pm 0.24$ & 3.1 & K4 (G7..K6) & $0.32(0.09 . .1 .40)$ \\
\hline $\mathrm{J} 3424648+2522306$ & $18.65 \pm 0.27$ & $17.22 \pm 0.17$ & $1.27 \pm 0.32$ & $0.99 \pm 0.20$ & $1.67 \pm 0.20$ & $1.98 \pm 0.20$ & 1.9 & M2 (M1..M3) & $0.62(0.21 . .1 .18)$ \\
\hline $\mathrm{J} 3424653+2538242$ & $18.03 \pm 0.26$ & $17.20 \pm 0.17$ & $0.51 \pm 0.31$ & $1.15 \pm 0.19$ & $1.96 \pm 0.18$ & $2.00 \pm 0.20$ & 7.5 & M0 (K7..M2) & $1.19(0.26 . .1 .46)$ \\
\hline $\mathrm{J} 3424660+2551194$ & $18.37 \pm 0.27$ & $17.08 \pm 0.17$ & $1.29 \pm 0.32$ & $1.37 \pm 0.18$ & $2.00 \pm 0.19$ & $2.19 \pm 0.20$ & 1.4 & M3 (M0..M3) & $0.04(0.04 . .1 .40)$ \\
\hline $\mathrm{J} 3424676+2529504$ & $15.94 \pm 0.26$ & $15.34 \pm 0.17$ & $0.35 \pm 0.31$ & $0.76 \pm 0.17$ & $1.00 \pm 0.17$ & $1.06 \pm 0.18$ & 2.6 & $\mathrm{~K} 0$ (G3..K3) & $0.91(0.41 . .1 .47)$ \\
\hline $\mathrm{J} 3424706+2528078$ & $15.57 \pm 0.26$ & $14.89 \pm 0.17$ & $0.47 \pm 0.31$ & $0.88 \pm 0.17$ & $1.24 \pm 0.17$ & $1.28 \pm 0.17$ & 2.6 & $\mathrm{~K} 3(\mathrm{~K} 0 . . \mathrm{K} 7)$ & $0.81(0.03 . .1 .27)$ \\
\hline $\mathrm{J} 3424741+2550196$ & $19.01 \pm 0.27$ & $17.82 \pm 0.18$ & $1.18 \pm 0.33$ & $1.55 \pm 0.21$ & $2.03 \pm 0.24$ & $2.20 \pm 0.27$ & 3.2 & M3 (M0..M3) & $0.06(0.06 . .1 .32)$ \\
\hline $\mathrm{J} 3424748+2536080$ & $17.32 \pm 0.26$ & $16.50 \pm 0.17$ & $0.46 \pm 0.31$ & $0.67 \pm 0.19$ & $1.10 \pm 0.19$ & $1.56 \pm 0.19$ & 4.0 & K3 (K2..M0) & $1.36(0.21 . .1 .42)$ \\
\hline $\mathrm{J} 3424751+2531461$ & $17.35 \pm 0.26$ & $16.28 \pm 0.17$ & $0.88 \pm 0.31$ & $0.87 \pm 0.18$ & $1.33 \pm 0.18$ & $1.41 \pm 0.19$ & 3.0 & K7 (K3..M0) & $0.69(0.08 . .1 .19)$ \\
\hline $\mathrm{J} 3424755+2527057$ & $17.64 \pm 0.26$ & $16.57 \pm 0.17$ & $0.79 \pm 0.31$ & $1.11 \pm 0.18$ & $1.71 \pm 0.18$ & $1.88 \pm 0.18$ & 1.8 & M0 (M0..M2) & $1.06(0.58 . .1 .35)$ \\
\hline $\mathrm{J} 3424760+2520247$ & $19.80 \pm 0.31$ & $18.40 \pm 0.19$ & $1.22 \pm 0.37$ & $1.95 \pm 0.23$ & $2.35 \pm 0.25$ & $2.46 \pm 0.29$ & 7.8 & M4 (M3..M5) & $0.68(0.00 . .1 .33)$ \\
\hline $\mathrm{J} 3424767+2553587$ & $17.73 \pm 0.26$ & $16.31 \pm 0.17$ & $1.28 \pm 0.31$ & $1.33 \pm 0.17$ & $1.99 \pm 0.18$ & $2.23 \pm 0.18$ & 1.4 & M3 (M1..M3) & $0.55(0.32 . .1 .36)$ \\
\hline $\mathrm{J} 3424792+2538413$ & $18.52 \pm 0.27$ & $17.67 \pm 0.17$ & $0.40 \pm 0.32$ & $0.74 \pm 0.24$ & $1.34 \pm 0.24$ & $1.71 \pm 0.25$ & 2.8 & K4 (K3..M1) & $1.68(0.04 . .1 .68)$ \\
\hline $\mathrm{J} 3424793+2519534$ & $18.10 \pm 0.26$ & $17.00 \pm 0.17$ & $0.82 \pm 0.32$ & $0.54 \pm 0.21$ & $1.16 \pm 0.22$ & $1.85 \pm 0.21$ & 8.0 & K7 (K3..M2) & $1.06(0.09 . .1 .43)$ \\
\hline $\mathrm{J} 3424801+2538059$ & $16.66 \pm 0.26$ & $16.05 \pm 0.17$ & $0.48 \pm 0.31$ & $0.92 \pm 0.17$ & $1.24 \pm 0.18$ & $1.23 \pm 0.19$ & 3.5 & K3 (G7..K6) & $0.49(0.04 . .1 .30)$ \\
\hline $\mathrm{J} 3424808+2520236$ & $17.33 \pm 0.26$ & $16.35 \pm 0.17$ & $0.57 \pm 0.31$ & $0.10 \pm 0.19$ & $0.66 \pm 0.19$ & $0.93 \pm 0.20$ & 5.9 & G6 (F9..K3) & $1.50(0.61 . .1 .50)$ \\
\hline $\mathrm{J} 3424821+2525220$ & $19.48 \pm 0.28$ & $16.93 \pm 0.17$ & $2.34 \pm 0.33$ & $2.08 \pm 0.17$ & $2.65 \pm 0.17$ & $2.87 \pm 0.18$ & 2.4 & M6 (M6..M6) & $0.83(0.49 . .1 .15)$ \\
\hline
\end{tabular}




\begin{tabular}{|c|c|c|c|c|c|c|c|c|c|}
\hline \multirow[b]{2}{*}{ 2MASS-ID } & \multicolumn{2}{|c|}{ measured magnitudes } & & extinction c & ected colors & & & & \\
\hline & Rmag & Imag & R-I & I-J & $\mathrm{I}-\mathrm{H}$ & $\mathrm{I}-\mathrm{K}_{s}$ & $\chi^{2}$ & SpT & $\mathrm{Av}$ \\
\hline J3424843+2552134 & $16.27 \pm 0.26$ & $15.43 \pm 0.17$ & $0.47 \pm 0.31$ & $0.21 \pm 0.17$ & $0.69 \pm 0.18$ & $0.61 \pm 0.18$ & 2.3 & G0 (F5..G9) & $1.41(0.72 . .1 .47)$ \\
\hline $\mathrm{J} 3424872+2538485$ & $18.74 \pm 0.27$ & $17.66 \pm 0.17$ & $0.66 \pm 0.32$ & $0.67 \pm 0.24$ & $1.21 \pm 0.27$ & $1.91 \pm 0.23$ & 6.2 & K7 (K5...M2) & $1.55(0.45 . .1 .55)$ \\
\hline $\mathrm{J} 3424883+2527402$ & $17.70 \pm 0.26$ & $17.14 \pm 0.17$ & $0.43 \pm 0.31$ & $0.91 \pm 0.19$ & $1.43 \pm 0.22$ & $1.45 \pm 0.26$ & 1.6 & K4 (K2..K5) & $0.51(0.17 . .1 .20)$ \\
\hline $\mathrm{J} 3424895+2543282$ & $17.67 \pm 0.26$ & $17.20 \pm 0.17$ & $0.44 \pm 0.31$ & $0.95 \pm 0.21$ & $1.25 \pm 0.23$ & $1.58 \pm 0.27$ & 2.5 & K4 (G6..K4) & $0.10(0.10 . .1 .37)$ \\
\hline $\mathrm{J} 3424923+2551093$ & $17.60 \pm 0.26$ & $16.43 \pm 0.17$ & $0.58 \pm 0.31$ & $0.19 \pm 0.18$ & $0.60 \pm 0.19$ & $0.53 \pm 0.21$ & 3.4 & G0 (F6..G8) & $2.19(1.48 . .2 .19)$ \\
\hline J3424934+2554044 & $17.13 \pm 0.26$ & $16.60 \pm 0.17$ & $0.50 \pm 0.31$ & $0.73 \pm 0.18$ & $1.21 \pm 0.20$ & $1.35 \pm 0.23$ & 1.1 & K3 (G6..K3) & $0.12(0.12 . .1 .22)$ \\
\hline $\mathrm{J} 3424940+2539203$ & $20.02 \pm 0.32$ & $18.42 \pm 0.18$ & $1.41 \pm 0.37$ & $1.71 \pm 0.24$ & $2.22 \pm 0.26$ & $2.50 \pm 0.28$ & 2.5 & M4 (M3..M5) & $0.72(0.05 . .1 .39)$ \\
\hline $\mathrm{J} 3424957+2534420$ & $17.74 \pm 0.26$ & $17.09 \pm 0.17$ & $0.35 \pm 0.31$ & $1.41 \pm 0.18$ & $2.01 \pm 0.18$ & $2.00 \pm 0.19$ & 16.0 & M0 (K4..M2) & $1.12(0.29 . .1 .40)$ \\
\hline J3424971+2533014 & $14.94 \pm 0.26$ & $14.34 \pm 0.17$ & $0.48 \pm 0.31$ & $0.99 \pm 0.17$ & $1.36 \pm 0.17$ & $1.41 \pm 0.17$ & 3.0 & K4 (K0..K7) & $0.45(0.04 . .1 .20)$ \\
\hline J3424988+2537107 & $14.51 \pm 0.26$ & $13.74 \pm 0.17$ & $0.53 \pm 0.31$ & $0.91 \pm 0.17$ & $1.34 \pm 0.17$ & $1.42 \pm 0.17$ & 1.9 & K4 (K2..K7) & $0.90(0.43 . .1 .39)$ \\
\hline J3424996+2532232 & $16.85 \pm 0.26$ & $16.28 \pm 0.17$ & $0.44 \pm 0.31$ & $0.89 \pm 0.18$ & $1.38 \pm 0.18$ & $1.50 \pm 0.19$ & 1.4 & & $0.49(0.28 . .0 .96)$ \\
\hline $\mathrm{J} 3425007+2550143$ & $15.29 \pm 0.26$ & $14.78 \pm 0.17$ & $0.23 \pm 0.31$ & $0.55 \pm 0.17$ & $0.69 \pm 0.18$ & $0.64 \pm 0.17$ & 3.0 & F7 (F5..K1) & $1.05(0.02 . .1 .31)$ \\
\hline $\mathrm{J} 3425010+2543473$ & $17.37 \pm 0.26$ & $16.57 \pm 0.17$ & $0.47 \pm 0.31$ & $0.90 \pm 0.18$ & $1.16 \pm 0.19$ & $1.33 \pm 0.20$ & 3.0 & K3 (K0..K7) & $1.26(0.50 . .1 .48)$ \\
\hline $\mathrm{J} 3425020+2546598$ & $16.51 \pm 0.26$ & $15.88 \pm 0.17$ & $0.40 \pm 0.31$ & $0.71 \pm 0.18$ & $0.97 \pm 0.18$ & $1.07 \pm 0.19$ & 2.0 & K0 (G4..K4) & $0.88(0.08 . .1 .44)$ \\
\hline $\mathrm{J} 3425025+2548082$ & $15.46 \pm 0.26$ & $14.77 \pm 0.17$ & $0.52 \pm 0.31$ & $0.92 \pm 0.17$ & $1.36 \pm 0.17$ & $1.40 \pm 0.17$ & 2.0 & K4 (K1..K7) & $0.68(0.31 . .1 .49)$ \\
\hline $\mathrm{J} 3425029+2528277$ & $15.25 \pm 0.26$ & $14.66 \pm 0.17$ & $0.59 \pm 0.31$ & $-1.78 \pm 0.22$ & $-0.41 \pm$ & $-0.02 \pm$ & 49.2 & & $0 . .1 .48)$ \\
\hline $\mathrm{J} 3425030+2521438$ & $19.10 \pm 0.27$ & $17.92 \pm 0.18$ & $0.77 \pm 0.33$ & $1.17 \pm 0.21$ & $1.63 \pm 0.24$ & $1.95 \pm 0.25$ & 2.5 & M0 (M0..M3) & $1.54(0.02 . .1 .54)$ \\
\hline $\mathrm{J} 3425049+2535089$ & $19.89 \pm 0.31$ & $18.64 \pm 0.20$ & $0.97 \pm 0.36$ & $1.88 \pm 0.23$ & $2.70 \pm 0.23$ & $2.54 \pm 0.31$ & 16.0 & & $1.08(0.20 . .1 .24)$ \\
\hline $\mathrm{J} 3425059+2537184$ & $15.64 \pm 0.26$ & $14.77 \pm 0.17$ & $0.53 \pm 0.31$ & $0.17 \pm 0.17$ & $0.62 \pm 0.18$ & $0.61 \pm 0.17$ & 3.0 & G0 (F7..G9) & $1.27(0.53 . .1 .37)$ \\
\hline $\mathrm{J} 3425061+2523290$ & $18.98 \pm 0.27$ & $17.51 \pm 0.17$ & $1.29 \pm 0.32$ & $1.39 \pm 0.19$ & $1.90 \pm 0.20$ & $2.27 \pm 0.21$ & 1.6 & M3 (M1..M3) & $0.68(0.39 . .1 .50)$ \\
\hline $\mathrm{J} 3425077+2521055$ & $12.76 \pm 0.26$ & $12.09 \pm 0.17$ & $0.27 \pm 0.31$ & $0.40 \pm 0.17$ & $0.52 \pm 0.17$ & $0.50 \pm 0.17$ & 1.7 & F5 (F5..G8) & $1.50(0.47 . .1 .50)$ \\
\hline $\mathrm{J} 3425086+2550261$ & $19.33 \pm 0.29$ & $18.12 \pm 0.18$ & $0.71 \pm 0.34$ & $0.93 \pm 0.26$ & $1.63 \pm 0.25$ & $2.18 \pm 0.24$ & 4.5 & M3) & $80 . .1 .89)$ \\
\hline $\mathrm{J} 3425090+2532153$ & $16.33 \pm 0.26$ & $15.40 \pm 0.17$ & $0.76 \pm 0.31$ & $0.75 \pm 0.18$ & $1.35 \pm 0.18$ & $1.65 \pm 0.18$ & 1.7 & K7 (K2..K7) & $0.67(0.67 . .1 .42)$ \\
\hline $\mathrm{J} 3425116+2550484$ & $17.45 \pm 0.26$ & $16.84 \pm 0.17$ & $0.50 \pm 0.31$ & $0.87 \pm 0.19$ & $1.43 \pm 0.19$ & $1.35 \pm 0.23$ & 1.8 & K4 (K1..K6) & $0.40(0.19 . .1 .18)$ \\
\hline $\mathrm{J} 3425124+2540423$ & $15.91 \pm 0.26$ & $15.34 \pm 0.17$ & $0.53 \pm 0.31$ & $0.92 \pm 0.17$ & $1.33 \pm 0.17$ & $1.41=$ & 2.1 & K4 (1 & 3..1.06) \\
\hline $\mathrm{J} 3425133+2531258$ & $15.33 \pm 0.26$ & $14.70 \pm 0.17$ & $0.47 \pm 0.31$ & $0.88 \pm 0.17$ & $1.23 \pm 0.17$ & $1.28 \pm 0.17$ & 2.6 & $\mathrm{~K} 3(\mathrm{C}$ & $0.59(0.01 . .1 .47)$ \\
\hline J3425167+2546137 & $18.94 \pm 0.27$ & $17.86 \pm 0.18$ & $0.62 \pm 0.32$ & $1.28 \pm$ & 1.7 & 23 & 4.9 & 13) & .72) \\
\hline J3425197+2544103 & $18.52 \pm 0.27$ & $17.56 \pm 0.17$ & $0.58 \pm 0.32$ & $1.03 \pm 0.20$ & $1.88 \pm 0.20$ & $2.08 \pm 0.20$ & 5.4 & M0 & 1.41 \\
\hline $\mathrm{J} 3425261+2536578$ & $17.70 \pm 0.26$ & $16.22 \pm 0.17$ & $1.16 \pm 0.31$ & $1.44 \exists$ & $2.06 \pm 0.17$ & $2.25 \pm 0.17$ & 3.3 & M3 & 1.21 \\
\hline $\mathrm{J} 3425293+2536250$ & $18.74 \pm 0.27$ & $17.84 \pm 0.18$ & $0.46 \pm 0.32$ & $1.23 \pm 0.20$ & & $2.13 \pm 0.22$ & 8. & & 1.66 \\
\hline $\mathrm{J} 3425313+2547057$ & 18.75 & $17.68=$ & $1.07 \pm 0.32$ & 1.07 & 1.56 & $1.66 \pm$. & 1.5 & M1 & 0.00 \\
\hline $\mathrm{J} 3425335+2524117$ & $17.43 \pm 0.26$ & $16.65 \pm 0.17$ & $0.43 \pm 0.31$ & $0.62 \pm 0.18$ & $0.89 \pm 0.20$ & $1.16 \pm 0.20$ & 2.0 & K0 ( & 1.34 \\
\hline $\mathrm{J} 3425337+2528013$ & $17.36 \pm 0.26$ & 16.5 & $0.43 \pm 0.31$ & $0.91 \pm 0.17$ & $1.38 \pm 0.18$ & $1.50 \pm 0.19$ & 1.5 & K4 & 1.55 \\
\hline $\mathrm{J} 3425344+2533583$ & $17.88 \pm 0.26$ & $17.27 \pm 0.17$ & $0.44 \pm 0.32$ & $0.84 \pm 0.20$ & $0.92 \pm$ & $1.55 \pm$ & 1.1 & K4 (C & $0.61(0.16 . .1 .49)$ \\
\hline $\mathrm{J} 3425357+2538116$ & $18.31 \pm 0.27$ & $17.72 \pm 0.17$ & $0.39 \pm 0.32$ & $0.89 \pm 0.22$ & $1.17 \pm 0.30$ & $1.78 \pm 0.29$ & 3.9 & K4 & $0.76(0.04 . .1 .49)$ \\
\hline $\mathrm{J} 3425360+2547585$ & $19.33 \pm 0.28$ & $19.13 \pm 0.22$ & $-0.34 \pm 0.36$ & $2.05 \pm 0.26$ & $2.43 \pm 0.29$ & $2.80 \pm$ & 37.6 & M0 (K2..M5) & $.2 .05)$ \\
\hline $\mathrm{J} 3425383+2542467$ & $14.77 \pm 0.26$ & $14.27 \pm 0.17$ & $0.37 \pm 0.31$ & $0.75 \pm 0.17$ & $1.00 \pm 0.17$ & $1.05 \pm 0.17$ & 2.6 & 5..K2) & $3 . .1 .41)$ \\
\hline $\mathrm{J} 3425404+2533163$ & $17.70 \pm 0.26$ & $17.22 \pm 0.17$ & $0.05 \pm 0.31$ & $1.20 \pm 0.18$ & $1.59 \pm 0.19$ & $1.64 \pm 0.21$ & 11.3 & K4 (K4...M2) & $1.62(0.07 . .1 .66)$ \\
\hline $\mathrm{J} 3425409+2541041$ & $16.75 \pm 0.26$ & $16.09 \pm 0.17$ & $0.41 \pm 0.31$ & $0.99 \pm 0.17$ & $1.39 \pm 0.18$ & $1.46 \pm 0.18$ & 2.7 & K6) & $0.97(0.56 . .1 .26)$ \\
\hline $\mathrm{J} 3425414+2527058$ & $14.52 \pm 0.26$ & $13.81 \pm 0.17$ & $0.41 \pm 0.31$ & $0.72 \pm 0.17$ & $0.97 \pm 0.17$ & $1.05 \pm 0.17$ & 2.3 & K0 (G5..K6) & $1.14(0.15 . .1 .47)$ \\
\hline J3425440+2546169 & $19.67 \pm 0.30$ & $18.20 \pm 0.18$ & $1.26 \pm 0.35$ & $1.37 \pm 0.22$ & $1.72 \pm 0.28$ & $2.45 \pm 0.25$ & 3.8 & M3 (M1..M4) & $0.79(0.18 . .1 .42)$ \\
\hline $\mathrm{J} 3425465+2523327$ & $18.80 \pm 0.27$ & $17.07 \pm 0.17$ & $1.73 \pm 0.32$ & $1.52 \pm 0.18$ & $2.02 \pm 0.19$ & $2.26 \pm 0.19$ & 2.0 & M4 (M3..M4) & $0.00(0.00 . .0 .86)$ \\
\hline $\mathrm{J} 3425470+2524063$ & $17.68 \pm 0.26$ & & $1.30 \pm 0.31$ & & & & 2. & & $0.09(0$ \\
\hline $\mathrm{J} 3425476+2537428$ & $18.65 \pm 0.27$ & $17.89 \pm 0.18$ & $0.57 \pm 0.32$ & $1.34 \pm 0.22$ & $1.84 \pm 0.24$ & $1.95 \pm 0.29$ & 5.7 & M0 (K3..M2) & $0.71(0.30 . .1 .48)$ \\
\hline J3425498+2534107 & $13.62 \pm 0.26$ & $13.01 \pm 0.17$ & $0.47 \pm 0.31$ & & & & 3.2 & & \\
\hline $\mathrm{J} 3425504+2524071$ & $19.94 \pm 0.31$ & $19.70=$ & $0.24 \pm 0.45$ & 3.6 & .34 & $4.70 \pm 0.34$ & 84.5 & M6. & $0.00(0$. \\
\hline $\mathrm{J} 3425505+2530385$ & & & $1.42 \pm 0.38$ & & & & 4.5 & & \\
\hline $\mathrm{J} 3425527+2551050$ & $16.84 \pm 0.26$ & $16.03 \pm 0.17$ & $0.45 \pm 0.31$ & $0.65 \pm 0.18$ & $0.94 \pm 0.18$ & $1.07 \pm 0.18$ & 1.7 & K0 (K0..K7) & $1.38(0.01 . .1 .47)$ \\
\hline $\mathrm{J} 3425528+2524519$ & $17.93 \pm 0.26$ & & $1.15 \pm 0.31$ & & & & 1. & & \\
\hline $\mathrm{J} 3425546+2544035$ & $13.77 \pm 0.26$ & $13.24 \pm 0.17$ & $0.38 \pm 0.31$ & $0.76 \pm 0.17$ & $0.99 \pm 0.17$ & $1.05 \pm 0.17$ & 2.7 & K0 (F7..K2) & $0.58(0.13 . .1 .49)$ \\
\hline $\mathrm{J} 3425550+2553410$ & $17.20 \pm 0.26$ & $16.45 \pm 0.17$ & $0.35 \pm 0.31$ & $0.39 \pm 0.18$ & \pm 0.20 & $=0.23$ & 1. & G3 (G0..K1) & $1.50(0.51 . .1 .59)$ \\
\hline $\mathrm{J} 3425554+2550536$ & $18.38 \pm 0.27$ & $17.27 \pm 0.17$ & $0.75 \pm 0.32$ & $1.13 \pm 0.19$ & $1.80 \pm 0.19$ & 1.83 & 2.5 & ..M2) & $1.34(0.57 . .1 .45)$ \\
\hline $\mathrm{J} 3425561+2539445$ & $12.16 \pm 0.26$ & $=0.17$ & $0.38 \pm 0.31$ & 0.7 & 0. & & 3. & K0 & $1 . .1 .40)$ \\
\hline $\mathrm{J} 3425586+2522007$ & $15.11 \pm 0.26$ & $14.30 \pm 0.17$ & $0.44 \pm 0.31$ & $0.68 \pm 0.17$ & $1.08 \pm 0.17$ & $1.09 \pm 0.17$ & 1.5 & K1 (K1..K7) & $1.42(0.10 . .1 .42)$ \\
\hline $\mathrm{J} 3425606+2547580$ & $15.24 \pm 0.26$ & $14.56 \pm 0.17$ & $0.49 \pm 0.31$ & $=0.17$ & \pm 0.17 & & 1. & $\mathrm{~K} 3 \mathrm{CG}_{8} \mathrm{KG}$ & $0.71(0.08 . .1 .43)$ \\
\hline $\mathrm{J} 3425617+2537172$ & $18.94 \pm 0.27$ & $17.70 \pm 0.17$ & $1.02 \pm 0.32$ & $1.07 \pm 0.21$ & $1.67 \pm 0.23$ & $1.93 \pm 0.26$ & 1.1 & M1 (M0..M2) & $0.83(0.34 . .1 .28)$ \\
\hline J3425640+2542197 & $17.09 \pm 0.26$ & $16.00 \pm 0.17$ & $0.71 \pm 0.31$ & $1.15 \pm 0.17$ & $1.81 \pm 0.17$ & $1.86 \pm 0.17$ & 3.3 & M0 (M0..M2) & $1.46(0.81 . .1 .49)$ \\
\hline-2528553 & $15.13 \pm 0.26$ & 14.32 & $0.44 \pm 0.31$ & $0.93 \pm 0.17$ & $1.41 \pm 0.17$ & $1.44 \pm 0.17$ & 2.0 & K4 & $1.39(0.06 . .1 .48)$ \\
\hline+2548591 & $19.24 \pm$ & & $1.14 \pm 0.33$ & $1.91 \pm 0.21$ & & $297+$ & 8.1 & M4 ( & $0.00(0.00 . .1 .47)$ \\
\hline $\mathrm{J} 3425680+2520528$ & $17.90 \pm 0.26$ & 17.10 & $0.80 \pm 0.32$ & $0.69=$ & 0.23 & & 1.2 & (.K7) & $0.00(0.00 . .1 .48)$ \\
\hline $\mathrm{J} 342$ & & & & & & 2 & 4. & & $0.1 .48)$ \\
\hline $\mathrm{J} 3425$ & 19.33 & & $1.26 \pm$ & & $=0.21$ & 0.23 & 2. & & 1.21( \\
\hline $\mathrm{J} 3425716+2524380$ & $18.42 \pm 0.27$ & 17.8 & $0.25 \pm 0.32$ & & & $1.92 \pm$ & 2.5 & $\mathrm{~K} 4$ & $1.15(0.24 . .1 .44)$ \\
\hline J34257 & & & & & & & 2.5 & & 49) \\
\hline $\mathrm{J} 3425724+2528233$ & 19.3 & & $1.07 \pm 0$ & $1.55 \pm$ & 1.99 & 2. & 5. & M4) & $1.27(0.03 . .1 .44)$ \\
\hline $\mathrm{J} 3425738+2525125$ & 17.4 & & 31 & & & & 1.3 & & .36) \\
\hline J3425754+2547106 & & & $5 \pm 0.31$ & & & & 2.1 & $\mathrm{~K} 4$ & 1.17( \\
\hline+2535466 & & & 37 & & & & 26 & M4) & 27) \\
\hline J3425784+2549406 & $18.18 \pm 0.27$ & $16.94 \pm 0.17$ & $1.06 \pm 0.32$ & $0.42 \pm 0.20$ & $=0.22$ & 21 & 7.3 & 2..K7) & $0.70(0.39 . .1 .47)$ \\
\hline $\mathrm{J} 3425792+2544195$ & & & & & & & 3.3 & & \\
\hline $\mathrm{J} 3425803+2519260$ & $16.92 \pm 0.26$ & $15.90 \pm 0.17$ & $0.83 \pm 0.31$ & $0.57 \pm 0.18$ & $1.26 \pm 0.19$ & $1.74 \pm 0.19$ & 5.2 & K7 (K2..M1) & $0.70(0.01 . .1 .42)$ \\
\hline J3425808+2525494 & $19.55 \pm 0.28$ & & $1.18 \pm 0.34$ & & & & 2.6 & M3 (M2..M4) & $0.77(0.21 . .1 .25)$ \\
\hline $\mathrm{J} 3425819+2525183$ & $17.54 \pm 0.26$ & $17.06 \pm 0.17$ & $5 \pm 0.31$ & $0.40 \pm 0.21$ & $0.72 \pm 0.28$ & \pm 0.31 & 1.2 & G6 (F6..G7) & $0.47(0.29 . .1 .01)$ \\
\hline +2522347 & & & & & & & 1.3 & G0 (F7..G6) & \\
\hline $\mathrm{J} 3425855+2539363$ & $15.21 \pm 0.26$ & $14.69 \pm 0.17$ & $0.50 \pm 0.31$ & $0.90 \pm 0.17$ & $1.21 \pm($ & $1.25 \pm 0$ & 3.2 & $\mathrm{~K} 3$ & $0.06\left(C_{1}-2\right)$ \\
\hline 5313 & & & & & & & 2.1 & & \\
\hline $\mathrm{J} 3425911+2536236$ & $18.25 \pm 0.26$ & $17.55 \pm 0.17$ & $0.31 \pm 0.32$ & $0.85 \pm 0.21$ & $1.32 \pm 0.24$ & $1.79 \pm 0.23$ & 4.3 & K4 (K4...M0) & $1.46(0.45 . .1 .46)$ \\
\hline $\begin{array}{r}+2520394 \\
\end{array}$ & $15.95 \pm 0.26$ & & $0.34 \pm 0$ & $0.43 \pm 0$ & & & 1.5 & F7 (F6..G9) & $1.49(0.83 . .1 .49)$ \\
\hline $\mathrm{J} 3425957+2530363$ & $19.86 \pm 0.31$ & $18.36 \pm 0.18$ & $1.50 \pm 0.36$ & $1.60 \pm 0.24$ & $2.15 \pm 0.30$ & & 1.4 & M4 (M2..M4) & $0.00(0.00 . .1 .37)$ \\
\hline $\mathrm{J} 34$ & 19 & & & & $2.16 \pm 0$ & & 4.1 & M3 (M2..M4) & $1.19(0.03 . .1 .40)$ \\
\hline $\mathrm{J} 3425979+2526221$ & $19.57 \pm 0.29$ & $17.93 \pm 0.18$ & $1.64 \pm 0.34$ & $1.56 \pm 0.21$ & $2.06 \pm 0.25$ & $2.39 \pm 0.26$ & 1.2 & M4 (M3..M4) & $0.00(0.00 . .0 .82)$ \\
\hline $\mathrm{J} 3425980+2544337$ & $16.23 \pm 0.26$ & $15.54 \pm 0.17$ & $0.42 \pm 0.31$ & $0.92 \pm 0.17$ & $1.39 \pm 0.17$ & $1.49 \pm 0.17$ & 1.7 & K4 (K3..K5) & $0.99(0.70 . .1 .33)$ \\
\hline
\end{tabular}




\begin{tabular}{|c|c|c|c|c|c|c|c|c|c|}
\hline \multirow[b]{2}{*}{ 2MASS-ID } & \multicolumn{2}{|c|}{ measured magnitudes } & & extinction cc & ected colors & & & & \\
\hline & Rmag & Imag & R-I & I-J & I-H & $\mathrm{I}-\mathrm{K}_{s}$ & $\chi^{2}$ & SpT & $\mathrm{Av}$ \\
\hline $\mathrm{J} 3425988+2543080$ & $17.58 \pm 0.26$ & $16.65 \pm 0.17$ & $0.59 \pm 0.31$ & $-0.18 \pm 0.21$ & $0.51 \pm$ & $0.16 \pm$ & 4.5 & F0 (B9..G5) & $1.26(0.28 . .1 .46)$ \\
\hline $\mathrm{J} 3430005+2546393$ & $15.73 \pm 0.26$ & $15.12 \pm 0.17$ & $0.37 \pm 0.31$ & $0.61 \pm 0.17$ & $0.82 \pm 0.17$ & $0.90 \pm 0.18$ & 1.9 & G7 (F6..K1) & $0.87(0.43 . .1 .48)$ \\
\hline $\mathrm{J} 3430023+2524230$ & $19.08 \pm 0.27$ & $17.79 \pm 0.18$ & $0.91 \pm 0.32$ & $1.30 \pm 0.20$ & $1.94 \pm 0.21$ & $2.04 \pm 0.22$ & 3.8 & M2 (M2..M4) & $1.44(0.12 . .1 .44)$ \\
\hline J3430049+2538317 & $15.18 \pm 0.26$ & $14.67 \pm 0.17$ & $0.49 \pm 0.31$ & $0.88 \pm 0.17$ & $1.20 \pm 0.17$ & $1.29 \pm 0.17$ & 2.6 & K3 (G0..K3) & $0.10(0.10 . .1 .49)$ \\
\hline $\mathrm{J} 3430069+2533387$ & $15.08 \pm 0.26$ & $14.44 \pm 0.17$ & $0.49 \pm 0.31$ & $0.88 \pm 0.17$ & $1.21 \pm 0.17$ & $1.28 \pm 0.17$ & 2.6 & K3 (G8..K4) & $0.57(0.16 . .1 .23)$ \\
\hline $\mathrm{J} 3430094+2546513$ & $17.42 \pm 0.26$ & $16.75 \pm 0.17$ & $0.45 \pm 0.31$ & $-0.14 \pm 0.23$ & $0.71 \pm 0.23$ & $1.30 \pm$ & 6.7 & F6 (A6..K1) & $0.81(0.22 . .1 .47)$ \\
\hline $\mathrm{J} 3430130+2529128$ & $16.56 \pm 0.26$ & $15.85 \pm 0.17$ & $0.51 \pm 0.31$ & $0.85 \pm 0.18$ & $1.23 \pm 0.18$ & $1.26 \pm 0.18$ & 2.2 & K3 (G8..K6) & $0.73(0.10 . .1 .46)$ \\
\hline $\mathrm{J} 3430132+2532372$ & $17.28 \pm 0.26$ & $16.62 \pm 0.17$ & $0.27 \pm 0.31$ & $0.47 \pm 0.19$ & $0.40 \pm 0.23$ & $0.88 \pm 0.22$ & 4.4 & F7 (F7..K3) & $1.48(0.39 . .1 .48)$ \\
\hline J3430154+2534379 & $15.17 \pm 0.26$ & $14.61 \pm 0.17$ & $0.32 \pm 0.31$ & $0.67 \pm 0.17$ & $0.84 \pm 0.17$ & $0.91 \pm 0.17$ & 2.8 & G7 (F7..K3) & $0.89(0.15 . .1 .24)$ \\
\hline $\mathrm{J} 3430165+2541383$ & $14.72 \pm 0.26$ & $13.99 \pm 0.17$ & $0.48 \pm 0.31$ & $0.95 \pm 0.17$ & $1.36 \pm 0.17$ & $1.43 \pm 0.17$ & 2.2 & K4 (K2..K6) & $0.96(0.67 . .1 .48)$ \\
\hline J3430186+2533021 & $14.79 \pm 0.26$ & $14.23 \pm 0.17$ & $0.32 \pm 0.31$ & $0.65 \pm 0.17$ & $0.88 \pm 0.17$ & $0.89 \pm 0.17$ & 2.4 & G7 (F8..K2) & $0.89(0.22 . .1 .48)$ \\
\hline J3430207+2552069 & $17.06 \pm 0.26$ & $16.29 \pm 0.17$ & $0.48 \pm 0.31$ & $0.73 \pm 0.18$ & $1.33 \pm 0.18$ & $1.28 \pm 0.19$ & 1.4 & K3 (K0..K7) & $1.10(0.47 . .1 .50)$ \\
\hline $\mathrm{J} 3430213+2549563$ & $17.84 \pm 0.26$ & $17.04 \pm 0.17$ & $0.45 \pm 0.31$ & $0.72 \pm 0.19$ & $1.52 \pm 0.19$ & $1.46 \pm 0.22$ & 2.1 & K4 (K4..M0) & $1.29(0.02 . .1 .44)$ \\
\hline $\mathrm{J} 3430220+2543556$ & $16.41 \pm 0.26$ & $15.85 \pm 0.17$ & $0.26 \pm 0.31$ & $0.46 \pm 0.18$ & $0.64 \pm 0.18$ & $0.30 \pm 0.22$ & 5.2 & F5 (A7..K0) & $2 . .1 .46)$ \\
\hline $\mathrm{J} 3430245+2547242$ & $16.12 \pm 0.26$ & $15.46 \pm 0.17$ & $0.32 \pm 0.31$ & $0.62 \pm 0.17$ & $0.89 \pm 0.18$ & $0.89 \pm 0.18$ & 2.0 & G7 (G5..K4) & $1.29(0.04 . .1 .49)$ \\
\hline $\mathrm{J} 3430246+2551532$ & $20.60 \pm 0.46$ & $18.98 \pm 0.23$ & $1.47 \pm 0.51$ & $2.80 \pm 0.25$ & $3.48 \pm 0.25$ & $3.56 \pm 0.27$ & 13.1 & M6.5 (M6..M8) & $0.57(0.02 . .1 .26)$ \\
\hline $\mathrm{J} 3430253+2553575$ & $16.47 \pm 0.26$ & $15.21 \pm 0.17$ & $0.82 \pm 0.31$ & $-0.11 \pm 0.18$ & $0.42 \pm 0.18$ & $0.60 \pm 0.18$ & 14.4 & G0 (F4..K0) & $1.64(0.50 . .1 .65)$ \\
\hline $\mathrm{J} 3430271+2524113$ & $18.10 \pm 0.26$ & $17.42 \pm 0.17$ & $0.34 \pm 0.32$ & $0.66 \pm 0.21$ & $0.95 \pm 0.25$ & $1.20 \pm 0.28$ & 1.6 & K0 (G9..K7) & $1.29(0.27 . .1 .34)$ \\
\hline J3430274+2545329 & $18.15 \pm 0.26$ & $17.71 \pm 0.17$ & $0.21 \pm 0.32$ & $1.27 \pm 0.20$ & $1.42 \pm 0.26$ & $1.46 \pm 0.32$ & 8.9 & K4 (K0..M0) & $0.86(0.04 . .1 .35)$ \\
\hline $\mathrm{J} 3430276+2544063$ & $17.96 \pm 0.26$ & $16.94 \pm 0.17$ & $1.01 \pm 0.31$ & $0.24 \pm 0.22$ & $1.19 \pm 0.22$ & $1.74 \pm 0.22$ & 12.4 & K7 (G2..K7) & $0.07(0.06 . .1 .47)$ \\
\hline $\mathrm{J} 3430277+2520035$ & $17.57 \pm 0.26$ & $16.83 \pm 0.17$ & $0.37 \pm 0.31$ & $0.52 \pm 0.19$ & $0.86 \pm 0.21$ & $0.68 \pm$ & 1.1 & G7 (G5..K7) & $1.41(0.00 . .1 .41)$ \\
\hline $\mathrm{J} 3430298+2553461$ & $15.64 \pm 0.26$ & $14.90 \pm 0.17$ & $0.52 \pm 0.31$ & $0.89 \pm 0.17$ & $1.36 \pm 0.17$ & $1.41 \pm 0.17$ & 1.7 & K4 (K0..K5) & $0.81(0.72 . .1 .47)$ \\
\hline $\mathrm{J} 3430331+2526338$ & $16.90 \pm 0.26$ & $16.24 \pm 0.17$ & $0.28 \pm 0.31$ & $0.54 \pm 0.18$ & $0.74 \pm 0.19$ & $0.51 \pm 0.21$ & 3.9 & F7 (F6..K3) & $1.41(0.26 . .1 .41)$ \\
\hline $\mathrm{J} 3430338+2548349$ & $16.18 \pm 0.26$ & $15.65 \pm 0.17$ & $0.26 \pm 0.31$ & $0.44 \pm 0.17$ & $0.65 \pm 0.18$ & $0.31 \pm 0.21$ & 4.9 & F5 (A4..K0) & $1.00(0.15 . .1 .46)$ \\
\hline $\mathrm{J} 3430341+2534111$ & $18.18 \pm 0.26$ & $16.99 \pm 0.17$ & $1.03 \pm 0.32$ & $0.38 \pm 0.22$ & $1.03 \pm 0.25$ & $1.72 \pm 0.23$ & 10.1 & K7 (G9..M2) & $0.58(0.02 . .1 .33)$ \\
\hline J3430364+2535286 & $17.15 \pm 0.26$ & $16.53 \pm 0.17$ & $0.39 \pm 0.31$ & $0.98 \pm 0.18$ & $1.40 \pm 0.19$ & $1.48 \pm 0.20$ & 2.4 & K4 (K2..K5) & $0.87(0.57 . .1 .30)$ \\
\hline $\mathrm{J} 3430391+2520185$ & $16.32 \pm 0.26$ & $15.49 \pm 0.17$ & $0.46 \pm 0.31$ & $0.34 \pm 0.18$ & $0.53 \pm 0.18$ & $0.65 \pm 0.18$ & 1.8 & F9 (F6..G7) & $1.40(0.83 . .1 .56)$ \\
\hline $\mathrm{J} 3430399+2536417$ & $19.31 \pm 0.28$ & $17.63 \pm 0.17$ & $1.61 \pm 0.33$ & $1.22 \pm 0.21$ & $1.76 \pm 0.23$ & $2.03 \pm 0.26$ & 3.0 & M3 (M1..M3) & $0.26(0.02 . .1 .48)$ \\
\hline $\mathrm{J} 3430419+2532554$ & $16.09 \pm 0.26$ & $15.47 \pm 0.17$ & $0.25 \pm 0.31$ & $0.38 \pm 0.18$ & $0.54 \pm 0.18$ & $0.50 \pm 0.19$ & 1.6 & F5 (F5..G8) & $1.37(0.36 . .1 .37)$ \\
\hline $\mathrm{J} 3430435+2538305$ & $17.76 \pm 0.26$ & $17.17 \pm 0.17$ & $0.29 \pm 0.31$ & $0.85 \pm 0.19$ & $0.89 \pm 0.24$ & $0.80 \pm$ & 4.3 & K0 (G3..K5) & $1.13(0.08 . .1 .44)$ \\
\hline $\mathrm{J} 3430444+2554269$ & $20.29 \pm 0.27$ & $19.95 \pm 0.24$ & $0.34 \pm 0.36$ & $5.20 \pm 0.24$ & $5.68 \pm 0.25$ & $5.77 \pm 0.25$ & 125.3 & L4.5 (M8..L5) & $0.00(0.00 . .1 .43)$ \\
\hline J3430483+2547227 & $18.38 \pm 0.27$ & $17.98 \pm 0.18$ & $0.26 \pm 0.32$ & $1.21 \pm 0.22$ & $1.22 \pm 0.35$ & $1.55 \pm$ & 6.4 & K4 (G5..K7) & $0.53(0.53 . .1 .46)$ \\
\hline $\mathrm{J} 3430502+2549076$ & $20.11 \pm 0.35$ & $18.10 \pm 0.18$ & $1.61 \pm 0.39$ & $0.60 \pm 0.30$ & $1.49 \pm 0.27$ & $1.88 \pm 0.26$ & 7.5 & M2 (M2..M4) & $1.49(0.04 . .1 .50)$ \\
\hline $\mathrm{J} 3430522+2535144$ & $17.56 \pm 0.26$ & $16.71 \pm 0.17$ & $0.41 \pm 0.31$ & $0.62 \pm 0.18$ & $0.87 \pm 0.20$ & $0.74 \pm 0.24$ & 3.0 & G7 (G5..K7) & $1.64(0.10 . .1 .64)$ \\
\hline $\mathrm{J} 3430539+2547423$ & $17.08 \pm 0.26$ & $16.46 \pm 0.17$ & $0.48 \pm 0.31$ & $0.79 \pm 0.18$ & $1.27 \pm 0.19$ & $1.29 \pm 0.20$ & 1.4 & K3 (G9..K4) & $0.51(0.39 . .1 .48)$ \\
\hline $\mathrm{J} 3430553+2534447$ & $17.98 \pm 0.26$ & $16.92 \pm 0.17$ & $0.53 \pm 0.32$ & $1.26 \pm 0.18$ & $1.90 \pm 0.18$ & $1.95 \pm 0.18$ & 7.8 & M0 (M0..M3) & $1.98(0.83 . .1 .98)$ \\
\hline J3430567+2549397 & $18.14 \pm 0.26$ & $17.04 \pm 0.17$ & $0.84 \pm 0.32$ & $0.54 \pm 0.21$ & $1.18 \pm 0.23$ & $1.79 \pm 0.21$ & 6.2 & K7 (K3..M1) & $0.96(0.03 . .1 .43)$ \\
\hline $\mathrm{J} 3430570+2534096$ & $15.32 \pm 0.26$ & $14.74 \pm 0.17$ & $0.51 \pm 0.31$ & $0.96 \pm 0.17$ & $1.29 \pm 0.17$ & $1.45 \pm 0.17$ & 2.7 & K4 (G7..K4) & $0.28(0.23 . .1 .50)$ \\
\hline J3430589+2535394 & $17.31 \pm 0.26$ & $16.38 \pm 0.17$ & $0.81 \pm 0.31$ & $0.56 \pm 0.19$ & $1.24 \pm 0.21$ & $1.78 \pm 0.19$ & 5.9 & K7 (K3..M0) & $0.46(0.20 . .1 .29)$ \\
\hline J3430598+2523492 & $15.53 \pm 0.26$ & $14.66 \pm 0.17$ & $0.43 \pm 0.31$ & $0.56 \pm 0.17$ & $0.81 \pm 0.17$ & $0.87 \pm 0.17$ & 1.7 & G7 (G6..K7) & $1.67(0.11 . .1 .68)$ \\
\hline $\mathrm{J} 3430602+2535516$ & $17.47 \pm 0.26$ & $16.80 \pm 0.17$ & $0.37 \pm 0.31$ & $-0.01 \pm 0.23$ & $1.18 \pm$ & $1.65 \pm$ & 1.6 & F0 (A0..G6) & $1.12(0.13 . .1 .41)$ \\
\hline $\mathrm{J} 3430607+2530401$ & $16.70 \pm 0.26$ & $15.96 \pm 0.17$ & $0.34 \pm 0.31$ & $0.58 \pm 0.17$ & $0.86 \pm 0.18$ & $0.92 \pm 0.19$ & 1.5 & G7 (G7..K7) & $1.51(0.04 . .1 .51)$ \\
\hline $\mathrm{J} 3430648+2546008$ & $18.65 \pm 0.27$ & $17.93 \pm 0.18$ & $0.24 \pm 0.32$ & $1.04 \pm 0.21$ & $1.50 \pm 0.24$ & $1.60 \pm 0.27$ & 3.8 & K4 (K4..M1) & $1.83(0.42 . .1 .83)$ \\
\hline $\mathrm{J} 3430653+2540528$ & $7.99 \pm 0.26$ & $7.52 \pm 0.17$ & $0.43 \pm 0.31$ & $1.18 \pm 0.17$ & $1.33 \pm 0.17$ & $1.37 \pm 0.17$ & 8.4 & $\mathrm{~K} 4(\mathrm{G} 2 . . \mathrm{K} 7)$ & $0.14(0.01 . .1 .48)$ \\
\hline $\mathrm{J} 3430677+2546100$ & $18.31 \pm 0.27$ & $17.42 \pm 0.17$ & $0.83 \pm 0.32$ & $0.60 \pm 0.23$ & $1.48 \pm 0.24$ & $1.63 \pm$ & 1.9 & K7 (G8..K7) & $0.19(0.19 . .1 .48)$ \\
\hline $\mathrm{J} 3430687+2528373$ & $19.27 \pm 0.28$ & $17.83 \pm 0.18$ & $1.30 \pm 0.33$ & $1.35 \pm 0.21$ & $2.14 \pm 0.21$ & $2.04 \pm 0.25$ & 3.0 & M3 (M1..M3) & $0.52(0.21 . .1 .47)$ \\
\hline J3430693+2553595 & $16.38 \pm 0.26$ & $15.64 \pm 0.17$ & $0.50 \pm 0.31$ & $0.74 \pm 0.17$ & $1.23 \pm 0.18$ & $1.34 \pm 0.18$ & 1.1 & K3 (K1..K7) & $0.93(0.16 . .1 .47)$ \\
\hline $\mathrm{J} 3430699+2549383$ & $15.25 \pm 0.26$ & $14.60 \pm 0.17$ & $0.39 \pm 0.31$ & $0.56 \pm 0.17$ & $0.89 \pm 0.17$ & $0.85 \pm 0.17$ & 1.6 & G7 (F6..K3) & $0.98(0.02 . .1 .49)$ \\
\hline $\mathrm{J} 3430706+2551363$ & $20.53 \pm 0.50$ & $17.66 \pm 0.18$ & $2.86 \pm 0.54$ & $1.79 \pm 0.19$ & $2.24 \pm 0.21$ & $2.50 \pm 0.21$ & 12.5 & M6 (M3..M6) & $0.00(0.00 . .1 .50)$ \\
\hline J3430714+2530397 & $17.41 \pm 0.26$ & $16.46 \pm 0.17$ & $0.91 \pm 0.31$ & $1.06 \pm 0.18$ & $1.68 \pm 0.18$ & $1.78 \pm 0.19$ & 1.4 & M0 (K4..M1) & $0.16(0.06 . .1 .49)$ \\
\hline $\mathrm{J} 3430726+2535490$ & $17.86 \pm 0.26$ & $17.47 \pm 0.17$ & $0.05 \pm 0.32$ & $1.15 \pm 0.19$ & $1.53 \pm 0.22$ & $1.76 \pm 0.22$ & 9.9 & K4 (K3..M1) & $1.26(0.13 . .1 .49)$ \\
\hline $\mathrm{J} 3430735+2533439$ & $15.65 \pm 0.26$ & $15.05 \pm 0.17$ & $0.34 \pm 0.31$ & $0.77 \pm 0.17$ & $1.01 \pm 0.17$ & $1.07 \pm 0.17$ & 2.8 & K0 (G6..K5) & $0.99(0.12 . .1 .42)$ \\
\hline J3430764+2531106 & $19.20 \pm 0.28$ & $19.55 \pm 0.26$ & $-0.90 \pm 0.38$ & $2.54 \pm 0.30$ & $2.48 \pm 0.38$ & $3.26 \pm 0.31$ & 83.3 & M0 (K0..M5) & $2.07(0.18 . .2 .07)$ \\
\hline $\mathrm{J} 3430779+2529351$ & $15.76 \pm 0.26$ & $14.96 \pm 0.17$ & $0.48 \pm 0.31$ & $0.85 \pm 0.17$ & $1.25 \pm 0.17$ & $1.27 \pm 0.17$ & 2.2 & K3 (K1..K6) & $1.21(0.50 . .1 .58)$ \\
\hline $\mathrm{J} 3430789+2548262$ & $19.04 \pm 0.28$ & $17.83 \pm 0.18$ & $1.16 \pm 0.33$ & $1.55 \pm 0.21$ & $2.04 \pm 0.23$ & $2.22 \pm 0.25$ & 3.4 & M3 (M0..M3) & $0.20(0.20 . .1 .26)$ \\
\hline J3430802+2522337 & $18.37 \pm 0.27$ & $17.55 \pm 0.17$ & $0.44 \pm 0.32$ & $0.61 \pm 0.22$ & $0.85 \pm 0.29$ & $1.28 \pm 0.25$ & 2.4 & K1 (K0..K7) & $1.45(0.06 . .1 .46)$ \\
\hline $\mathrm{J} 3430817+2526374$ & $15.96 \pm 0.26$ & $15.02 \pm 0.17$ & $0.90 \pm 0.31$ & $1.06 \pm 0.17$ & $1.66 \pm 0.17$ & $1.81 \pm 0.17$ & 1.4 & M0 (K4..M1) & $0.15(0.04 . .1 .12)$ \\
\hline $\mathrm{J} 3430832+2537090$ & $14.59 \pm 0.26$ & $13.98 \pm 0.17$ & $0.37 \pm 0.31$ & $0.76 \pm 0.17$ & $0.97 \pm 0.17$ & $1.08 \pm 0.17$ & 2.7 & K0 (G0..K3) & $0.93(0.40 . .1 .59)$ \\
\hline J3430845+2550486 & $15.33 \pm 0.26$ & $14.69 \pm 0.17$ & $0.25 \pm 0.31$ & $0.38 \pm 0.17$ & $0.50 \pm 0.17$ & $0.54 \pm 0.17$ & 1.4 & F5 (F5..G8) & $1.47(0.67 . .1 .47)$ \\
\hline $\mathrm{J} 3430862+2534236$ & $17.77 \pm 0.26$ & $16.91 \pm 0.17$ & $0.69 \pm 0.31$ & $1.06 \pm 0.18$ & $1.71 \pm 0.19$ & $2.04 \pm 0.19$ & 3.3 & M0 (K4..M0) & $0.64(0.52 . .1 .46)$ \\
\hline J3430876+2535046 & $18.53 \pm 0.27$ & $18.16 \pm 0.19$ & $-0.08 \pm 0.33$ & $1.25 \pm 0.23$ & $1.61 \pm 0.27$ & $1.90 \pm 0.26$ & 12.9 & K4 (K4..M1) & $1.69(0.52 . .1 .69)$ \\
\hline J3430913+2549588 & $18.72 \pm 0.27$ & $17.62 \pm 0.18$ & $0.58 \pm 0.33$ & $1.24 \pm 0.19$ & $1.89 \pm 0.20$ & $1.92 \pm 0.21$ & 5.6 & M0 (M0..M3) & $1.96(0.42 . .1 .96)$ \\
\hline J3430937+2553589 & $17.94 \pm 0.26$ & $17.32 \pm 0.17$ & $0.56 \pm 0.31$ & $0.56 \pm 0.23$ & $1.42 \pm 0.22$ & $1.71 \pm 0.24$ & 3.8 & K5 (G7..K7) & $0.23(0.05 . .1 .39)$ \\
\hline $\mathrm{J} 3430955+2532000$ & $17.04 \pm 0.26$ & $16.17 \pm 0.17$ & $0.87 \pm 0.31$ & $1.11 \pm 0.17$ & $1.64 \pm 0.18$ & $1.80 \pm 0.18$ & 1.9 & M0 (K2..M0) & $0.00(0.00 . .1 .48)$ \\
\hline J3430979+2531155 & $17.47 \pm 0.26$ & $16.68 \pm 0.17$ & $0.38 \pm 0.31$ & $1.00 \pm 0.18$ & $1.46 \pm 0.18$ & $1.44 \pm 0.19$ & 3.1 & K4 (K4..M0) & $1.55(0.38 . .1 .55)$ \\
\hline J3430981+2520213 & $17.95 \pm 0.26$ & $17.05 \pm 0.17$ & $0.52 \pm 0.32$ & $0.58 \pm 0.19$ & $1.01 \pm 0.21$ & $1.27 \pm 0.21$ & 1.6 & $\mathrm{~K} 2(\mathrm{~K} 2 . . \mathrm{K} 7)$ & $1.43(0.34 . .1 .43)$ \\
\hline J3430989+2524528 & $12.76 \pm 0.26$ & $12.10 \pm 0.17$ & $0.26 \pm 0.31$ & $0.36 \pm 0.17$ & $0.43 \pm 0.17$ & $0.43 \pm 0.17$ & 1.9 & F4 (F4..G7) & $1.47(0.39 . .1 .47)$ \\
\hline J3431018+2521268 & $17.45 \pm 0.26$ & $16.69 \pm 0.17$ & $0.38 \pm 0.31$ & $0.76 \pm 0.19$ & $0.95 \pm 0.20$ & $1.06 \pm 0.21$ & 2.6 & K0 (K0..K7) & $1.42(0.11 . .1 .42)$ \\
\hline $\mathrm{J} 3431038+2533460$ & $19.36 \pm 0.28$ & $18.24 \pm 0.18$ & $0.61 \pm 0.34$ & $1.19 \pm 0.23$ & $1.84 \pm 0.23$ & $1.99 \pm 0.24$ & 3.7 & M0 (M0..M2) & $1.94(1.12 . .1 .94)$ \\
\hline J3431040+2535497 & $16.57 \pm 0.26$ & $15.72 \pm 0.17$ & $0.53 \pm 0.31$ & $0.87 \pm 0.17$ & $1.36 \pm 0.18$ & $1.41 \pm 0.18$ & 1.5 & K4 (K4..M0) & $1.17(0.10 . .1 .31)$ \\
\hline J3431046+2548024 & $15.67 \pm 0.26$ & $15.10 \pm 0.17$ & $0.28 \pm 0.31$ & $0.46 \pm 0.17$ & $0.61 \pm 0.17$ & $0.71 \pm 0.18$ & 1.6 & F7 (F4..G8) & $1.08(0.63 . .1 .44)$ \\
\hline J3431078+2537527 & $19.41 \pm 0.29$ & $18.08 \pm 0.18$ & $1.17 \pm 0.34$ & $1.12 \pm 0.24$ & $1.80 \pm 0.25$ & $1.90 \pm 0.29$ & 1.1 & M2 (M0..M3) & $0.61(0.08 . .1 .29)$ \\
\hline $\mathrm{J} 3431090+2528191$ & $19.34 \pm 0.28$ & $18.04 \pm 0.18$ & $1.23 \pm 0.33$ & $1.06 \pm 0.25$ & $1.63 \pm 0.29$ & $2.01 \pm 0.29$ & 1.5 & M2 (M0..M2) & $0.27(0.02 . .0 .90)$ \\
\hline J3431097+2543585 & $19.25 \pm 0.28$ & $18.70 \pm 0.20$ & $0.14 \pm 0.34$ & $1.69 \pm 0.25$ & $2.07 \pm 0.29$ & $2.42 \pm$ & 16.4 & M0 (K3..M4) & $1.55(0.52 . .1 .55)$ \\
\hline $\mathrm{J} 3431099+2525030$ & $16.33 \pm 0.26$ & $15.48 \pm 0.17$ & $0.49 \pm 0.31$ & $-0.00 \pm 0.18$ & $0.18 \pm 0.18$ & $0.06 \pm 0.19$ & 4.5 & F0 (A8..F6) & $1.40(0.73 . .1 .48)$ \\
\hline J3431103+2551185 & $11.05 \pm 0.26$ & $9.13 \pm 0.17$ & $1.62 \pm 0.31$ & $1.80 \pm 0.17$ & $2.62 \pm 0.17$ & $2.86 \pm 0.17$ & 5.5 & M5 (M5..M6) & $1.12(0.27 . .1 .46)$ \\
\hline $\mathrm{J} 3431122+2541538$ & $15.27 \pm 0.26$ & $14.59 \pm 0.17$ & $0.49 \pm 0.31$ & $-0.24 \pm 0.17$ & $0.15 \pm 0.18$ & $0.26 \pm 0.18$ & 7.8 & F0 (A4..G3) & $0.74(0.06 . .1 .34)$ \\
\hline $\mathrm{J} 3431145+2524455$ & $18.32 \pm 0.27$ & $16.96 \pm 0.17$ & $1.32 \pm 0.32$ & $1.02 \pm 0.19$ & $1.59 \pm 0.19$ & $1.97 \pm 0.20$ & 2.7 & M2 (K6..M2) & $0.15(0.15 . .1 .46)$ \\
\hline J3431146+2529474 & $18.61 \pm 0.27$ & $17.61 \pm 0.17$ & $0.62 \pm 0.32$ & $1.22 \pm 0.20$ & $1.70 \pm 0.20$ & $2.06 \pm 0.21$ & 5.0 & M0 (M0..M3) & $1.43(0.18 . .1 .43)$ \\
\hline $\mathrm{J} 3431152+2521410$ & $17.39 \pm 0.26$ & $16.37 \pm 0.17$ & $0.61 \pm 0.31$ & $0.67 \pm 0.18$ & $1.02 \pm 0.19$ & $0.91 \pm 0.19$ & 4.5 & K1 (K1..K7) & $1.54(0.13 . .1 .60)$ \\
\hline $\mathrm{J} 3431156+2525231$ & $16.45 \pm 0.26$ & $14.52 \pm 0.17$ & $1.78 \pm 0.31$ & $1.50 \pm 0.17$ & $2.06 \pm 0.17$ & $2.28 \pm 0.17$ & 2.3 & M4 (M3..M4) & $0.55(0.28 . .1 .30)$ \\
\hline $\mathrm{J} 3431172+2531407$ & $16.15 \pm 0.26$ & $15.34 \pm 0.17$ & $0.41 \pm 0.31$ & $0.29 \pm 0.17$ & $0.42 \pm 0.18$ & $0.46 \pm 0.18$ & 2.2 & F5 (F5..G6) & $1.50(0.76 . .1 .50)$ \\
\hline
\end{tabular}


T. Eisenbeiss et al.: New brown dwarf candidates in the Pleiades

\begin{tabular}{|c|c|c|c|c|c|c|c|c|c|}
\hline \multirow[b]{2}{*}{ 2MASS-ID } & \multicolumn{2}{|c|}{ measured magnitudes } & & extinction $\mathrm{cc}$ & ected colors & & & & \\
\hline & Rmag & Imag & R-I & I-J & $\mathrm{I}-\mathrm{H}$ & $\mathrm{I}-\mathrm{K}_{s}$ & $\chi^{2}$ & SpT & $\mathrm{Av}$ \\
\hline $\mathrm{J} 3431195+2532535$ & $15.49 \pm 0.26$ & $14.85 \pm 0.17$ & $0.24 \pm 0.31$ & $0.50 \pm 0.17$ & $0.66 \pm 0.17$ & $0.69 \pm 0.17$ & 1.9 & F7 (F6..K2) & $1.52(0.30 . .1 .52)$ \\
\hline J3431197+2547282 & $16.96 \pm 0.26$ & $15.94 \pm 0.17$ & $1.02 \pm 0.31$ & $1.03 \pm 0.17$ & $1.63 \pm 0.18$ & $1.84 \pm 0.18$ & 1.5 & M1 (K3..M1) & $0.00(0.00 . .1 .43)$ \\
\hline $\mathrm{J} 3431202+2542253$ & $15.53 \pm 0.26$ & $14.87 \pm 0.17$ & $0.46 \pm 0.31$ & $0.92 \pm 0.17$ & $1.40 \pm 0.17$ & $1.44 \pm 0.17$ & 1.8 & K4 (K2..K6) & $0.74(0.49 . .1 .29)$ \\
\hline $\mathrm{J} 3431222+2531220$ & $17.98 \pm 0.26$ & $17.00 \pm 0.17$ & $0.46 \pm 0.32$ & $0.32 \pm 0.20$ & $0.73 \pm 0.21$ & $1.16 \pm$ & 1.3 & G5 (F8..K1) & $1.96(1.14 . .1 .99)$ \\
\hline $\mathrm{J} 3431246+2550073$ & $12.65 \pm 0.26$ & $12.08 \pm 0.17$ & $0.23 \pm 0.31$ & $0.44 \pm 0.17$ & $0.48 \pm 0.17$ & $0.54 \pm 0.17$ & 2.2 & F5 (F3..G8) & $1.29(0.27 . .1 .50)$ \\
\hline J3431260+2551464 & $15.71 \pm 0.26$ & $14.92 \pm 0.17$ & $0.31 \pm 0.31$ & $0.11 \pm 0.17$ & $0.23 \pm 0.17$ & $0.19 \pm 0.18$ & 1.6 & F0 (A6..F4) & $1.80(1.38 . .1 .89)$ \\
\hline $\mathrm{J} 3431270+2524262$ & $17.19 \pm 0.26$ & $16.01 \pm 0.17$ & $0.65 \pm 0.31$ & $-0.12 \pm 0.18$ & $0.01 \pm 0.20$ & $0.04 \pm 0.22$ & 8.7 & F0 (A3..G3) & $1.98(0.65 . .1 .99)$ \\
\hline J3431281+2520042 & $18.34 \pm 0.27$ & $17.14 \pm 0.17$ & $1.13 \pm 0.32$ & $1.21 \pm 0.19$ & $1.74 \pm 0.19$ & $1.96 \pm 0.21$ & 1.5 & M2 (M0..M2) & $0.26(0.04 . .0 .91)$ \\
\hline J3431292+2535196 & $17.51 \pm 0.26$ & $16.87 \pm 0.17$ & $0.36 \pm 0.31$ & $0.59 \pm 0.19$ & $0.84 \pm 0.22$ & $0.89 \pm 0.24$ & 1.6 & G7 (F8..K3) & $1.06(0.10 . .1 .42)$ \\
\hline $\mathrm{J} 3431300+2519372$ & $19.86 \pm 0.31$ & $18.83 \pm 0.27$ & $0.74 \pm 0.42$ & $1.80 \pm 0.32$ & $2.31 \pm 0.34$ & $2.62 \pm 0.34$ & 9.4 & M3 (M0..M4) & $1.08(0.15 . .1 .50)$ \\
\hline J3431306+2524374 & $16.14 \pm 0.26$ & $15.29 \pm 0.17$ & $0.46 \pm 0.31$ & $0.60 \pm 0.17$ & $1.01 \pm 0.17$ & $1.04 \pm 0.17$ & 1.4 & K0 (K0..K7) & $1.50(0.35 . .1 .50)$ \\
\hline J3431308+2547048 & $17.53 \pm 0.26$ & $16.75 \pm 0.17$ & $0.48 \pm 0.31$ & $0.81 \pm 0.19$ & $1.22 \pm 0.19$ & $1.33 \pm 0.20$ & 1.4 & K3 (K0..K6) & $1.14(0.40 . .1 .47)$ \\
\hline J3431315+2524115 & $20.26 \pm 0.35$ & $18.57 \pm 0.20$ & $1.36 \pm 0.41$ & $1.15 \pm 0.29$ & $2.00 \pm 0.27$ & $2.23 \pm 0.29$ & 1.6 & M3 (M3..M4) & $1.26(0.20 . .1 .48)$ \\
\hline $\mathrm{J} 3431329+2554024$ & $18.81 \pm 0.26$ & $18.14 \pm 0.17$ & $0.19 \pm 0.32$ & $1.07 \pm 0.22$ & $1.16 \pm$ & $1.71 \pm$ & 3.4 & K4 (K1..M1) & $1.81(0.31 . .1 .88)$ \\
\hline $\mathrm{J} 3431356+2542273$ & $16.35 \pm 0.26$ & $15.52 \pm 0.17$ & $0.49 \pm 0.31$ & $0.84 \pm 0.17$ & $1.39 \pm 0.17$ & $1.46 \pm 0.17$ & 1.1 & K4 (K4...M0) & $1.29(0.04 . .1 .44)$ \\
\hline $\mathrm{J} 3431360+2541479$ & $15.67 \pm 0.26$ & $15.10 \pm 0.17$ & $0.41 \pm 0.31$ & $0.71 \pm 0.17$ & $0.93 \pm 0.17$ & $1.09 \pm 0.18$ & 2.3 & K0 (F8..K2) & 0.63 \\
\hline $\mathrm{J} 3431362+2528401$ & $16.68 \pm 0.26$ & $15.99 \pm 0.17$ & $0.51 \pm 0.31$ & $0.87 \pm 0.18$ & $1.34 \pm 0.18$ & $1.46 \pm 0.18$ & 1.3 & K4 (K3..K6) & $0.69(0.37 . .1 .19)$ \\
\hline J3431411+2544547 & $19.24 \pm 0.28$ & $17.70 \pm 0.17$ & $1.54 \pm 0.33$ & $0.87 \pm$ & $1.49 \pm 0.28$ & $2.28 \pm 0.23$ & 5.1 & M3 (M0..M3) & $.00 . .1 .00)$ \\
\hline J3431424+2530508 & $13.07 \pm 0.26$ & $12.40 \pm 0.17$ & $0.37 \pm 0.31$ & $0.63 \pm 0.17$ & $0.86 \pm 0.17$ & $0.86 \pm$ & 2.3 & G7 & 1.12 \\
\hline $\mathrm{J} 3431428+2543291$ & $14.04 \pm 0.26$ & $13.40 \pm 0.17$ & $0.38 \pm$ & $0.60 \pm 0.17$ & $0.87 \pm 0.17$ & $0.86=$ & 2.0 & & \\
\hline $\mathrm{J} 3431431+2536090$ & $18.81 \pm 0.27$ & $18.25 \pm 0.19$ & $0.13 \pm 0.33$ & $1.01 \pm 0.26$ & $1.63 \pm 0.28$ & $1.75 \pm 0.29$ & 5.1 & K4 & ..1.65) \\
\hline J3431466+2547474 & $18.99 \pm 0.28$ & $17.44 \pm 0.17$ & $1.56 \pm 0.33$ & $0.81 \pm 0.23$ & $1.64 \pm 0.23$ & $1.41=$ & 8.7 & M2 (K3..M2) & $0.1 .36)$ \\
\hline $\mathrm{J} 3431476+2533135$ & $15.73 \pm 0.26$ & $15.05 \pm 0.17$ & $0.30 \pm 0.31$ & $0.54 \pm 0.17$ & $0.73 \pm 0.17$ & $0.76 \pm 0.17$ & 2.0 & 1..K1) & $3 . .1 .47)$ \\
\hline J3431496+2548192 & $15.03 \pm 0.26$ & $14.45 \pm 0.17$ & $0.27 \pm 0.31$ & & $0.68 \pm 0.17$ & $0.63 \pm 0.17$ & 2.3 & & \\
\hline $\mathrm{J} 3431513+2526206$ & $17.89 \pm 0.26$ & $16.76 \pm 0.17$ & $0.76 \pm 0.31$ & $1.18 \pm 0.18$ & $1.71 \pm 0.18$ & $1.86 \pm 0.18$ & 2.7 & Mo (N & 1.38 \\
\hline $\mathrm{J} 3431529+2531517$ & $16.35 \pm 0.26$ & $15.73 \pm 0.17$ & $0.32 \pm 0.31$ & $0.68 \pm 0.17$ & $0.85 \pm 0.18$ & & 2.9 & & \\
\hline $\mathrm{J} 3431532+2552557$ & $16.97 \pm 0.26$ & $16.31 \pm 0.17$ & $0.40 \pm 0.31$ & $0.57 \pm 0.18$ & $0.87 \pm 0.19$ & $0.83=$ & 1.8 & G7 (F8..K2) & $1.02(0.50 . .1 .49)$ \\
\hline J3431571+2541504 & $19.11 \pm 0.28$ & $18.20 \pm 0.20$ & $0.55 \pm 0.34$ & & & & 5. & & \\
\hline J3431573+2520296 & $15.11 \pm 0.26$ & $13.69 \pm 0.17$ & $1.38 \pm 0.31$ & $1.33 \pm 0.17$ & $1.95 \pm 0.17$ & $2.14 \pm 0.17$ & 1.1 & M3 (N & $0.14(0.07 . .0 .87)$ \\
\hline J3431593+2529049 & $19.42 \pm 0.28$ & $17.70 \pm 0.17$ & $1.46 \pm 0.33$ & & & & 4.3 & & \\
\hline $\mathrm{J} 3431619+2535041$ & $17.05 \pm 0.26$ & $16.36 \pm 0.17$ & $0.37 \pm 0.31$ & $0.63 \pm 0.18$ & $0.87 \pm 0.19$ & 0.8 & 2.4 & G7 ( & 1.16 \\
\hline $\mathrm{J} 3431630+2525230$ & $19.65 \pm 0.29$ & $18.35 \pm 0.18$ & $0.89 \pm 0.35$ & $1.15 \pm$ & 1.6 & & 2.0 & M3) & 1.5 \\
\hline $\mathrm{J} 3431642+2551184$ & $15.38 \pm 0.26$ & $14.72 \pm 0.17$ & $0.37 \pm 0.31$ & $0.63 \pm 0.17$ & $0.84 \pm 0.17$ & $0.89 \pm 0.17$ & 2.1 & G7 (G0..K3) & $2.1 .42)$ \\
\hline $\mathrm{J} 3431647+2542229$ & $18.96 \pm 0.28$ & $18.02 \pm 0.18$ & $0.51 \pm 0.33$ & $1.14 \pm$ & $1.96 \pm 0.22$ & & 6.1 & & 61) \\
\hline J3431674+2537156 & $15.71 \pm 0.26$ & $15.17 \pm 0.17$ & $0.26 \pm 0.31$ & $0.45 \pm 0.17$ & $0.43 \pm 0.18$ & $0.55 \pm 0.18$ & 2.6 & F5 (A8..G9) & $1.03(0.18 . .1 .47)$ \\
\hline $\mathrm{J} 3431683+2532076$ & $15.01 \pm 0.26$ & $14.18 \pm 0.17$ & $0.48 \pm 0.31$ & $0.91 \pm 0.17$ & $1.39 \pm 0.17$ & & 1.8 & & 1.29 \\
\hline J3431688+2527047 & $16.08 \pm 0.26$ & $15.28 \pm 0.17$ & $0.42 \pm 0.31$ & $0.71 \pm 0.17$ & $1.07 \pm 0.17$ & $1.11 \pm 0.18$ & 1.7 & K1 (K1..K7) & $1.45(0.28 . .1 .45)$ \\
\hline J3431692+2538382 & $16.50 \pm 0.26$ & $15.89 \pm 0.17$ & $0.35 \pm 0.31$ & $0.63 \pm 0.18$ & $0.80 \pm 0.18$ & $0.94 \pm 0.19$ & 2.3 & & \\
\hline J3431721+2533534 & $17.83 \pm 0.26$ & $17.34 \pm 0.17$ & $0.41 \pm 0.32$ & 0.20 & $1.18 \pm 0.25$ & $1.56 \pm 0.25$ & 4.9 & K4 (G6..K4) & $0.32(0.14 . .1 .47)$ \\
\hline $\mathrm{J} 3431725+2530085$ & $20.97 \pm 0.55$ & $20.10 \pm 0.38$ & $0.83 \pm 0.67$ & 3. & 3.8 & 4.1 & 20.7 & M6.5 (M4..M8.5) & 0.13 \\
\hline J3431741+2535087 & $16.35 \pm 0.26$ & $15.71 \pm 0.17$ & $0.42 \pm 0.31$ & $0.67 \pm 0.17$ & $0.99 \pm 0.18$ & $1.05 \pm 0.18$ & 1.6 & K0 (G1...K2) & $0.86(0.50 . .1 .50)$ \\
\hline $\mathrm{J} 3431744+2543410$ & $17.64 \pm 0.26$ & $17.23 \pm 0.17$ & $0.39 \pm 0.31$ & $1.00 \pm 0.20$ & $1.32 \pm 0.24$ & & 2.6 & K4 (G9..K4) & $0.08(0$. \\
\hline J3431761+2523134 & $16.97 \pm 0.26$ & $16.28 \pm 0.17$ & $0.30 \pm 0.31$ & $0.50 \pm 0.18$ & $0.63 \pm 0.19$ & $0.63 \pm 0.20$ & 2.1 & F7 (F7..K1) & $1.45(0.47 . .1 .49)$ \\
\hline $\mathrm{J} 3431782+2534040$ & $17.11 \pm 0.26$ & $16.40 \pm 0.17$ & $0.50 \pm 0.31$ & $0.85 \pm 0.18$ & $1.44 \pm 0.18$ & 14 & 1.6 & K4 (K3..K6) & $0.81(0.37 . .1 .01)$ \\
\hline $\mathrm{J} 3431818+2531470$ & $18.31 \pm 0.27$ & $16.85 \pm 0.17$ & $1.31 \pm 0.32$ & $1.34 \pm 0.18$ & $1.98 \pm 0.18$ & $2.19 \pm 0.18$ & 1.2 & M3 (M2..M3) & $0.59(0.36 . .1 .47)$ \\
\hline $\mathrm{J} 3431827+2523314$ & $14.95 \pm 0.26$ & $14.23 \pm 0.17$ & $0.36 \pm 0.31$ & $0.49 \pm 0$ & 07 & 7 & 1.5 & G4 (I & $.1 .46)$ \\
\hline $\mathrm{J} 3431835+2537075$ & $18.88 \pm 0.27$ & $17.92 \pm 0.18$ & $0.71 \pm 0.33$ & $1.10 \pm 0.23$ & $1.80 \pm 0.23$ & $1.99=$ & 1.9 & M0 (K6..M2) & $0.91(0.25 . .1 .41)$ \\
\hline $\mathrm{J} 3431847+2520249$ & $15.42 \pm 0.26$ & $14.48 \pm 0.17$ & $0.93 \pm 0.31$ & $1.08 \pm 0.17$ & $1.63 \pm 0.17$ & $179+0$ & 1.8 & M0 (K3..M1) & $0.03(0.03 . .1 .31)$ \\
\hline J3431848+2524426 & $16.94 \pm 0.26$ & $16.34 \pm 0.17$ & $0.21 \pm 0.31$ & $0.41 \pm 0.18$ & $0.51 \pm 0.20$ & $0.56=$ & 1.7 & F5 (F5..K1) & $1.48(0.08 . .1 .48)$ \\
\hline J3431882+2536482 & $17.55 \pm 0.26$ & $16.88 \pm 0.17$ & $0.42 \pm 0.32$ & $1.05 \pm 0.18$ & $1.34 \pm 0.20$ & $1.44 \pm 0.21$ & 3.8 & K4 (K1..M0) & $0.92(0.03 . .1 .43)$ \\
\hline J3431886+2527489 & $18.18 \pm 0.26$ & $17.57 \pm 0.18$ & $0.44 \pm 0.32$ & $0.90 \pm 0.22$ & $1.37 \pm 0.24$ & 1.73 & 1.3 & K4 ( & $0.64(0.49 . .1 .36)$ \\
\hline J3431907+2534031 & 18.76 & & $0.76 \pm c$ & & & \pm 0.25 & 3.6 & K7 & $0.94(0.26 . .1 .43)$ \\
\hline+2522439 & 18.53 & & 1. & & & & 1. & & \\
\hline $\mathrm{J} 3431923+2530152$ & $18.66 \pm 0.27$ & & $1.46 \pm 0$ & $0.96 \pm$ & 1.43 & 1.92 & 5.8 & M2 & 0.12 \\
\hline $\mathrm{J} 3431931+2533363$ & & & & & & & 7. & & \\
\hline J3431968+2527144 & $14.53 \pm 0.26$ & 17 & $0.55 \pm 0$ & 0. & 1.2 & 1.26 & 2. & K3 & $1.21(0$ \\
\hline J3431969+2530592 & $16.89 \pm 0.26$ & & 31 & & & & 3 & & \\
\hline $\mathrm{J} 3432027+2537340$ & $13.88 \pm 0.26$ & & $0.24 \pm$ & 0. & 17 & & 2.6 & F7 & $7.1 .47)$ \\
\hline J3432037+2519473 & $16.56 \pm$ & 15 & 31 & & 18 & & 3.7 & & \\
\hline $\mathrm{J} 3432112+2523516$ & $16.38 \pm 0.26$ & $15.56 \pm 0.17$ & $0.45 \pm 0.31$ & $0.55 \pm 0.17$ & $0.81 \pm 0.18$ & .18 & 1.8 & 6..K3) & $1.39(0.48 . .1 .48)$ \\
\hline J3432115+2540499 & & & & & & & 1.7 & & \\
\hline $\mathrm{J} 3432127+2528354$ & $20.98 \pm 0.53$ & $17.89 \pm 0.18$ & $2.96 \pm 0.55$ & $1.14 \pm 0.22$ & $1.66 \pm 0.25$ & $1.94 \pm 0.27$ & 23.5 & 0..M6) & $0.51(0.29 . .1 .43)$ \\
\hline $\mathrm{J} 3432139+2545103$ & $16.45 \pm 0.26$ & $15.80 \pm 0.17$ & $0.31 \pm 0.31$ & & & $=0.19$ & 3.2. & F5 (F4..K1) & $1.29(0.06 . .1 .29)$ \\
\hline $\mathrm{J} 3432143+2519535$ & $19.30 \pm 0.29$ & $17.53 \pm 0.19$ & $1.75 \pm 0.35$ & $1.55 \pm 0.20$ & $2.06 \pm 0.21$ & $2.25 \pm 0.22$ & 2.0 & M4 (M3..M4) & $0.06(0.06 . .1 .01)$ \\
\hline J3432153+2551301 & $17.84 \pm 0.26$ & & $0.49 \pm 0$ & & & & 3.1 & G7 (F9..K2) & \\
\hline $\mathrm{J} 3432165+2547531$ & $16.70 \pm 0.26$ & $15.82 \pm 0.17$ & $0.49 \pm 0.31$ & $0.32 \pm 0.18$ & $0.60 \pm 0.18$ & $0.59 \pm 0.18$ & 1.9 & (0..G6) & $1.47(1.01 . .1 .47)$ \\
\hline J3432 & & & & & & & 1.0 & & \\
\hline $\mathrm{J} 3432185+2523291$ & $15.70 \pm 0.26$ & $14.84 \pm 0.17$ & $0.49 \pm 0.31$ & & 0.17 & 1. & 2.0 & K0 & o..1.48) \\
\hline $\mathrm{J} 343$ & & & & & & & 1.2 & & $0.1 .67)$ \\
\hline $\mathrm{J} 34322$ & & & 2 & & & & 1.3 & & \\
\hline-2542078 & & & - & & 1.75 & 1.87 & 1.8 & M1 & 1.23( \\
\hline $\mathrm{J} 3432232+2526052$ & $13.74 \pm 0.26$ & $13.04 \pm 0.17$ & $3 \pm 0$ & \pm 0.17 & $0.64 \pm 0.17$ & & 1.8 & $\mathrm{~K} 1)$ & $1.39(0$ \\
\hline+2519355 & 18. & & & & & & 17.0 & $\mathrm{G} 0(\mathrm{~F} 2 . . \mathrm{K} 1)$ & $4 . .1 .51)$ \\
\hline J3432293+2527568 & $14.04 \pm 0.26$ & \pm 0.17 & $2 \pm 0.31$ & \pm 0.17 & & $=0.17$ & 1.8 & & $1.46(0.21 . .1 .46)$ \\
\hline $7+2530035$ & $16.39 \pm 0.26$ & $15.38 \pm 0$ & $0.58 \pm 0.31$ & $0.27 \pm$ & $0.01 \pm 0.21$ & $1.33 \pm$ & 3.9 & F0 (A5..G3) & $1.64(0.46 . .1 .64)$ \\
\hline $\mathrm{J} 3432320+2543567$ & & & & & & & 2.6 & & \\
\hline $\mathrm{J} 3432328+2545254$ & $16.73 \pm 0.26$ & $6 \pm 0$ & $0.48 \pm 0$. & $0.62 \pm 0.17$ & $0.95 \pm 0.18$ & & 1. & K0 (K0..K7) & $1.50(0.27 . .1 .50)$ \\
\hline $\mathrm{J} 3432332+2537259$ & $17.24 \pm 0.26$ & $16.41 \pm 0.17$ & $0.58 \pm 0.31$ & $0.80 \pm 0.18$ & & & 3.7 & K3 (G8..K7) & \\
\hline $\mathrm{J} 3432356+2551483$ & $6 \pm 0.26$ & $16.45 \pm 0$. & $0.59 \pm 0$ & $0.14 \pm 0.19$ & $0.63 \pm 0.20$ & $0.52 \pm 0.23$ & 3.9 & G0 (F7..G7) & $1.59(0.81 . .1 .59)$ \\
\hline J3432384+2530515 & $13.28 \pm 0.26$ & $12.63 \pm 0.17$ & & $0.51 \pm 0.17$ & $0.68 \pm 0.17$ & & 2.2 & F7 (F7..K3) & $1.50(0.12 . .1 .55)$ \\
\hline J3432388+2547185 & $14.97 \pm 0.26$ & $14.24 \pm 0.17$ & $0.36 \pm 0.31$ & $0.54 \pm 0.17$ & $0.72 \pm 0.17$ & $0.69 \pm 0.17$ & 2.3 & G4 (F7..K3) & $1.39(0.09 . .1 .43)$ \\
\hline $\mathrm{J} 3432389+2534453$ & $18.18 \pm 0.27$ & $16.83 \pm 0.17$ & $0.73 \pm 0.32$ & $0.20 \pm 0.19$ & $0.45 \pm 0.22$ & $0.42 \pm 0.24$ & 6.1 & G0 (F5..G9) & $2.35(1.53 . .2 .39)$ \\
\hline
\end{tabular}




\begin{tabular}{|c|c|c|c|c|c|c|c|c|c|}
\hline \multirow[b]{2}{*}{ 2MASS-ID } & \multicolumn{2}{|c|}{ measured magnitudes } & & extinction co & ected colors & & & & \\
\hline & Rmag & Imag & R-I & I-J & $\mathrm{I}-\mathrm{H}$ & $\mathrm{I}-\mathrm{K}_{s}$ & $\chi^{2}$ & SpT & $\mathrm{Av}$ \\
\hline $\mathrm{J} 3432393+2532303$ & $18.04 \pm 0.27$ & $16.90 \pm 0.17$ & $1.14 \pm 0.32$ & $1.11 \pm 0.18$ & $1.46 \pm 0.20$ & $1.80 \pm 0.21$ & 3.7 & $\mathrm{M} 1$ (K3..M1) & $0.00(0.00 . .1 .45)$ \\
\hline $\mathrm{J} 3432397+2552440$ & $18.75 \pm 0.27$ & $17.89 \pm 0.18$ & $0.52 \pm 0.32$ & $0.73 \pm 0.25$ & $1.17 \pm 0.31$ & $1.34 \pm 0.32$ & 1.1 & K3 (K3..K7) & $1.27(0.30 . .1 .27)$ \\
\hline $\mathrm{J} 3432417+2536191$ & $18.37 \pm 0.28$ & $17.26 \pm 0.17$ & $1.11 \pm 0.33$ & $1.20 \pm 0.19$ & $1.53 \pm 0.23$ & $1.66 \pm 0.25$ & 4.5 & M1 (K3..M1) & $0.00(0.00 . .1 .40)$ \\
\hline $\mathrm{J} 3432454+2551426$ & $16.08 \pm 0.26$ & $15.41 \pm 0.17$ & $0.38 \pm 0.31$ & $0.74 \pm 0.17$ & $1.00 \pm 0.18$ & $1.05 \pm 0.18$ & 2.4 & K0 (G7..K5) & $1.11(0.13 . .1 .45)$ \\
\hline $\mathrm{J} 3432482+2529191$ & $18.86 \pm 0.27$ & $18.14 \pm 0.18$ & $0.48 \pm 0.33$ & $1.33 \pm 0.23$ & $1.90 \pm 0.24$ & $2.17 \pm$ & 6.0 & M0 (K4..M3) & $0.90(0.02 . .1 .45)$ \\
\hline $\mathrm{J} 3432501+2541096$ & $19.26 \pm 0.29$ & $17.92 \pm 0.18$ & $1.03 \pm 0.34$ & $1.05 \pm 0.23$ & $1.52 \pm 0.26$ & $2.04 \pm 0.23$ & 2.3 & M1 (M0..M3) & $1.21(0.06 . .1 .21)$ \\
\hline $\mathrm{J} 3432513+2540089$ & $16.46 \pm 0.26$ & $15.83 \pm 0.17$ & $0.46 \pm 0.31$ & $-0.84 \pm 0.21$ & $-1.10 \pm 0.38$ & $-0.14 \pm \ldots$ & 11.1 & B0 (B0..B9) & $0.63(0.06 . .1 .37)$ \\
\hline $\mathrm{J} 3432530+2532474$ & $18.41 \pm 0.27$ & $17.41 \pm 0.17$ & $0.79 \pm 0.32$ & $0.67 \pm 0.22$ & $1.44 \pm 0.22$ & $1.58 \pm 0.23$ & 1.3 & K7 (K3..M0) & $0.83(0.06 . .1 .32)$ \\
\hline $\mathrm{J} 3432541+2548597$ & $15.89 \pm 0.26$ & $15.15 \pm 0.17$ & $0.49 \pm 0.31$ & $0.86 \pm 0.17$ & $1.25 \pm 0.17$ & $1.26 \pm 0.17$ & 2.4 & K3 (K0..K5) & $0.93(0.21 . .1 .24)$ \\
\hline $\mathrm{J} 3432590+2525510$ & $18.42 \pm 0.27$ & $16.95 \pm 0.17$ & $1.16 \pm 0.32$ & $1.47 \pm 0.18$ & $2.10 \pm 0.18$ & $2.20 \pm 0.18$ & 3.7 & M3 (M3..M4) & $1.16(0.03 . .1 .35)$ \\
\hline $\mathrm{J} 3432610+2545571$ & $15.89 \pm 0.26$ & $15.22 \pm 0.17$ & $0.50 \pm 0.31$ & $0.83 \pm 0.17$ & $1.25 \pm 0.17$ & $1.26 \pm 0.17$ & 2.0 & K3 (G8..K7) & $0.61(0.08 . .1 .47)$ \\
\hline $\mathrm{J} 3432615+2547105$ & $14.31 \pm 0.26$ & $13.57 \pm 0.17$ & $0.74 \pm 0.31$ & $-2.61 \pm 0.19$ & $-1.97 \pm 0.20$ & $-1.49 \pm 0.21$ & 228.2 & B0 (B0..B9) & $0.00(0.00 . .1 .49)$ \\
\hline $\mathrm{J} 3432621+2519364$ & $16.88 \pm 0.26$ & $16.04 \pm 0.17$ & $0.68 \pm 0.31$ & $1.24 \pm 0.17$ & $1.77 \pm 0.18$ & $1.88 \pm 0.18$ & 4.5 & M0 (K4..M1) & $0.60(0.22 . .1 .46)$ \\
\hline $\mathrm{J} 3432643+2542033$ & $14.29 \pm 0.26$ & $13.67 \pm 0.17$ & $0.22 \pm 0.31$ & $0.37 \pm 0.17$ & $0.53 \pm 0.17$ & $0.56 \pm 0.17$ & 1.3 & F5 (F5..G9) & $1.47(0.41 . .1 .47)$ \\
\hline $\mathrm{J} 3432658+2546331$ & $18.22 \pm 0.27$ & $17.58 \pm 0.18$ & $0.34 \pm 0.32$ & $0.55 \pm 0.23$ & $0.90 \pm 0.27$ & $1.48 \pm 0.26$ & 4.2 & K1 (G6..K7) & $1.16(0.11 . .1 .41)$ \\
\hline $\mathrm{J} 3432713+2549584$ & $17.95 \pm 0.27$ & $17.34 \pm 0.17$ & $0.41 \pm 0.32$ & $0.64 \pm 0.22$ & $0.88 \pm 0.28$ & $1.15 \pm 0.30$ & 1.6 & $\mathrm{~K} 0(\mathrm{G} 1 . . \mathrm{K} 4)$ & $10 . .1 .36)$ \\
\hline $\mathrm{J} 3432726+2521267$ & $18.43 \pm 0.27$ & $18.59 \pm 0.37$ & $-0.54 \pm 0.46$ & $3.38 \pm 0.37$ & $3.96 \pm 0.37$ & $4.08 \pm 0.37$ & 98.3 & M4 (M1..M7) & $1.44(0.18 . .1 .49)$ \\
\hline $\mathrm{J} 3432747+2540442$ & $16.64 \pm 0.26$ & $16.27 \pm 0.17$ & $0.29 \pm 0.31$ & $0.24 \pm 0.19$ & $0.50 \pm 0.21$ & $0.76 \pm 0.22$ & 1.9 & F6 (A4..G2) & $0.28(0.09 . .1 .37)$ \\
\hline $\mathrm{J} 3432754+2536049$ & $18.31 \pm 0.27$ & $17.52 \pm 0.18$ & $0.48 \pm 0.32$ & $0.75 \pm 0.22$ & $1.32 \pm 0.24$ & $1.28 \pm 0.27$ & 1.2 & $\mathrm{~K} 3(\mathrm{~K} 0 . . \mathrm{K} 7)$ & $1.18(0.38 . .1 .49)$ \\
\hline $\mathrm{J} 3432763+2551202$ & $18.70 \pm 0.27$ & $18.64 \pm 0.20$ & $-0.25 \pm 0.34$ & $1.94 \pm 0.23$ & $2.31 \pm 0.26$ & $2.48 \pm 0.28$ & 46.3 & M0 (K1..M4) & $.19 . .1 .46)$ \\
\hline $\mathrm{J} 3432776+2538515$ & $16.62 \pm 0.26$ & $15.94 \pm 0.17$ & $0.48 \pm 0.31$ & $0.85 \pm 0.17$ & $1.21 \pm 0.18$ & $1.31 \pm 0.18$ & 2.0 & K3 (G9..K7) & $0.74(0.22 . .1 .49)$ \\
\hline $\mathrm{J} 3432792+2536239$ & $18.25 \pm 0.27$ & $17.42 \pm 0.17$ & $0.70 \pm 0.32$ & $0.73 \pm 0.21$ & $1.49 \pm 0.23$ & $1.63 \pm 0.24$ & 1.4 & $\mathrm{~K} 7(\mathrm{~K} 2 . . \mathrm{K} 7)$ & $37 . .1 .23)$ \\
\hline $\mathrm{J} 3432797+2544219$ & $19.17 \pm 0.29$ & $18.48 \pm 0.21$ & $0.20 \pm 0.36$ & $1.70 \pm 0.24$ & $2.07 \pm 0.25$ & $2.07 \pm 0.30$ & 19.3 & M0 (K7..M4) & $1.85(0.41 . .1 .85)$ \\
\hline $\mathrm{J} 3432815+2534515$ & $17.64 \pm 0.26$ & $16.94 \pm 0.17$ & $0.31 \pm 0.31$ & $1.02 \pm 0.18$ & $1.55 \pm 0.19$ & $1.44 \pm 0.20$ & 4.4 & $\mathrm{~K} 4(\mathrm{~K} 4 . . \mathrm{N}$ & $.14 . .1 .49)$ \\
\hline $\mathrm{J} 3432824+2540418$ & $17.10 \pm 0.26$ & $16.79 \pm 0.17$ & $0.31 \pm 0.31$ & $0.08 \pm 0.22$ & $0.66 \pm 0.27$ & $1.11 \pm$ & 2.5 & F6 (A3..F7) & $0.00(0.00 . .0 .83)$ \\
\hline $\mathrm{J} 3432829+2535240$ & $16.69 \pm 0.26$ & $16.21 \pm 0.17$ & $0.33 \pm 0.31$ & $0.69 \pm 0.18$ & $0.81 \pm 0.19$ & $0.90 \pm 0.20$ & 3.1 & G7 (F6..K0) & $0.56(0.47 . .1 .35)$ \\
\hline $\mathrm{J} 3432830+2547267$ & $14.06 \pm 0.26$ & $13.30 \pm 0.17$ & $0.38 \pm 0.31$ & $0.70 \pm 0.17$ & $1.05 \pm 0.17$ & $1.03 \pm 0.17$ & 2.0 & $\mathrm{~K} 0(\mathrm{G} 9 .$. & $1.42(0.09 . .1 .48)$ \\
\hline $\mathrm{J} 3432834+2542538$ & $18.25 \pm 0.27$ & $17.49 \pm 0.17$ & $0.34 \pm 0.32$ & $0.96 \pm 0.20$ & $1.38 \pm 0.22$ & $1.61 \pm 0.23$ & 2.6 & K4 (K4..M0) & $1.59(0.42 . .1 .59)$ \\
\hline $\mathrm{J} 3432858+2530061$ & $16.83 \pm 0.26$ & $16.01 \pm 0.17$ & $0.42 \pm 0.31$ & $0.24 \pm 0.18$ & $0.54 \pm 0.19$ & $0.37 \pm 0.20$ & 2.8 & F5 (F5..G9) & $1.49(0.54 . .1 .49)$ \\
\hline $\mathrm{J} 3432879+2553456$ & $16.21 \pm 0.26$ & $15.46 \pm 0.17$ & $0.39 \pm 0.31$ & $0.75 \pm 0.17$ & $1.01 \pm 0.18$ & $1.02 \pm 0.17$ & 2.8 & $\mathrm{~K} 0(\mathrm{G} 7 . . \mathrm{K} 7)$ & $1.36(0.15 . .1 .42)$ \\
\hline $\mathrm{J} 3432885+2552256$ & $17.90 \pm 0.26$ & $17.18 \pm 0.17$ & $0.51 \pm 0.31$ & $0.91 \pm 0.20$ & $1.37 \pm 0.21$ & $1.37 \pm 0.25$ & 1.8 & $\mathrm{~K} 4$ (K2..K7) & $0.81(0.23 . .1 .50)$ \\
\hline $\mathrm{J} 3432909+2554182$ & $16.33 \pm 0.26$ & $18.46 \pm 0.17$ & $-2.31 \pm 0.31$ & $2.65 \pm 0.18$ & $2.77 \pm 0.21$ & $3.01 \pm 0.22$ & 335.4 & K4 (B9...M3) & $0.68(0.02 . .1 .49)$ \\
\hline $\mathrm{J} 3432920+2519081$ & $17.94 \pm 0.27$ & $19.12 \pm 0.43$ & $-1.64 \pm 0.51$ & $2.68 \pm 0.44$ & $3.29 \pm 0.44$ & $3.24 \pm 0.45$ & 73.3 & K4 (F4..M3) & $1.72(0.51 . .1 .72)$ \\
\hline $\mathrm{J} 3432941+2539138$ & $18.44 \pm 0.27$ & $17.34 \pm 0.17$ & $0.91 \pm 0.32$ & $0.49 \pm 0.23$ & $1.41 \pm 0.21$ & $1.54 \pm 0.24$ & 3.3 & K7 (K1..M0) & $0.70(0.15 . .1 .46)$ \\
\hline $\mathrm{J} 3432970+2536360$ & $18.77 \pm 0.27$ & $17.70 \pm 0.17$ & $0.88 \pm 0.32$ & $1.06 \pm 0.21$ & $1.81 \pm 0.22$ & $1.65 \pm 0.27$ & 2.6 & M0 (K5..M2) & $0.74(0.40 . .1 .49)$ \\
\hline $\mathrm{J} 3433000+2543504$ & $18.21 \pm 0.27$ & $16.72 \pm 0.17$ & $1.33 \pm 0.32$ & $1.38 \pm 0.18$ & $1.94 \pm 0.18$ & $2.18 \pm 0.18$ & 1.2 & M3 (M2..M3) & $0.61(0.38 . .1 .42)$ \\
\hline $\mathrm{J} 3433029+2525311$ & $17.98 \pm 0.26$ & $16.89 \pm 0.17$ & $0.54 \pm 0.31$ & $0.35 \pm 0.19$ & $0.49 \pm 0.23$ & $0.49 \pm 0.25$ & 3.0 & F9 (F6..G8) & $2.04(1.32 . .2 .10)$ \\
\hline $\mathrm{J} 3433045+2544591$ & $18.46 \pm 0.27$ & $16.93 \pm 0.17$ & $1.09 \pm 0.32$ & $0.22 \pm 0.20$ & $1.05 \pm 0.20$ & $1.76 \pm 0.19$ & 18.4 & $\mathrm{~K} 7$ (K4..M1) & $1.67(0.49 . .1 .67)$ \\
\hline $\mathrm{J} 3433079+2548476$ & $16.64 \pm 0.26$ & $15.99 \pm 0.17$ & $0.51 \pm 0.31$ & $0.90 \pm 0.17$ & $1.39 \pm 0.18$ & $1.40 \pm 0.18$ & 1.7 & $\mathrm{~K} 4$ (K0..K7) & $0.53(0.23 . .1 .21)$ \\
\hline $\mathrm{J} 3433089+2539486$ & $18.20 \pm 0.26$ & $17.03 \pm 0.17$ & $0.68 \pm 0.32$ & $0.32 \pm 0.20$ & $0.28 \pm 0.27$ & $0.42 \pm 0.27$ & 6.2 & F9 (F4..K6) & $1.83(0.07 . .1 .88)$ \\
\hline $\mathrm{J} 3433104+2552423$ & $18.19 \pm 0.26$ & $17.47 \pm 0.17$ & $0.44 \pm 0.31$ & $1.03 \pm 0.20$ & $1.56 \pm 0.21$ & $1.05 \pm 0.31$ & 7.4 & $\mathrm{~K} 4$ (K3..M1) & $1.08(0.26 . .1 .40)$ \\
\hline $\mathrm{J} 3433119+2529397$ & $17.49 \pm 0.26$ & $16.36 \pm 0.17$ & $0.84 \pm 0.31$ & $1.15 \pm 0.18$ & $1.67 \pm 0.18$ & $1.82 \pm 0.18$ & 2.2 & M0 (M0..M3) & $1.05(0.03 . .1 .22)$ \\
\hline $\mathrm{J} 3433131+2541020$ & $19.55 \pm 0.29$ & $18.06 \pm 0.18$ & $1.07 \pm 0.34$ & $0.57 \pm 0.28$ & $1.94 \pm \ldots$ & $2.09 \pm 0.23$ & 6.2 & M1 (M0..M4) & $1.59(0.04 . .1 .59)$ \\
\hline $\mathrm{J} 3433159+2544335$ & $18.42 \pm 0.27$ & $17.54 \pm 0.17$ & $0.51 \pm 0.32$ & $0.69 \pm 0.22$ & $1.16 \pm 0.24$ & $1.42 \pm 0.24$ & 1.4 & K3 (K3..M0) & $1.39(0.22 . .1 .49)$ \\
\hline $\mathrm{J} 3433163+2550228$ & $16.11 \pm 0.26$ & $15.30 \pm 0.17$ & $0.41 \pm 0.31$ & $0.68 \pm 0.17$ & $1.06 \pm 0.17$ & $0.99 \pm 0.18$ & 2.2 & K0 (K0..K7) & $1.49(0.03 . .1 .49)$ \\
\hline $\mathrm{J} 3433167+2549215$ & $18.34 \pm 0.27$ & $17.71 \pm 0.18$ & $0.26 \pm 0.33$ & $1.07 \pm 0.21$ & $1.50 \pm 0.22$ & $1.53 \pm 0.25$ & 4.1 & $\mathrm{~K} 4$ (K3...M1) & $1.37(0.02 . .1 .38)$ \\
\hline $\mathrm{J} 3433179+2533412$ & $22.16 \pm 1.62$ & $18.71 \pm 0.18$ & $3.13 \pm 1.63$ & $3.06 \pm 0.19$ & $3.20 \pm 0.20$ & $2.91 \pm 0.23$ & 15.1 & M7.5 (M6.5..M8.5) & $1.18(0.09 . .1 .18)$ \\
\hline $\mathrm{J} 3433183+2540327$ & $17.12 \pm 0.26$ & $16.44 \pm 0.17$ & $0.29 \pm 0.31$ & $0.25 \pm 0.19$ & $0.26 \pm 0.22$ & $0.43 \pm 0.24$ & 1.7 & $\mathrm{~F} 3(\mathrm{~F} 2 . . \mathrm{G} 4)$ & $1.46(0.52 . .1 .48)$ \\
\hline $\mathrm{J} 3433199+2533558$ & $16.74 \pm 0.26$ & $16.02 \pm 0.17$ & $0.11 \pm 0.31$ & $-1.15 \pm 0.22$ & $-0.46 \pm 0.22$ & $-0.65 \pm 0.27$ & 16.3 & B0 (B0..F2) & $2.29(0.46 . .2 .29)$ \\
\hline $\mathrm{J} 3433202+2534307$ & $14.21 \pm 0.26$ & $13.57 \pm 0.17$ & $0.40 \pm 0.31$ & $0.58 \pm 0.17$ & $0.86 \pm 0.17$ & $0.86 \pm 0.17$ & 1.7 & G7 (F8..K2) & $0.91(0.07 . .1 .49)$ \\
\hline $\mathrm{J} 3433220+2549004$ & $18.64 \pm 0.28$ & $17.74 \pm 0.18$ & $0.42 \pm 0.33$ & $1.45 \pm 0.19$ & $1.76 \pm 0.21$ & $2.10 \pm 0.20$ & 12.6 & M0 (M0..M4) & $1.79(0.16 . .1 .79)$ \\
\hline $\mathrm{J} 3433224+2524515$ & $16.69 \pm 0.26$ & $16.07 \pm 0.17$ & $0.38 \pm 0.31$ & $0.61 \pm 0.18$ & $0.94 \pm 0.18$ & $0.78 \pm 0.19$ & 3.0 & G7 (G0..K3) & $0.93(0.10 . .1 .21)$ \\
\hline $\mathrm{J} 3433243+2544198$ & $14.57 \pm 0.26$ & $13.95 \pm 0.17$ & $0.25 \pm 0.31$ & $0.51 \pm 0.17$ & $0.69 \pm 0.17$ & $0.64 \pm 0.17$ & 2.2 & F7 (F7..K2) & $1.36(0.02 . .1 .36)$ \\
\hline $\mathrm{J} 3433257+2553548$ & $18.31 \pm 0.26$ & $17.64 \pm 0.17$ & $0.34 \pm 0.31$ & $0.90 \pm 0.21$ & $1.52 \pm 0.22$ & $1.53 \pm 0.25$ & 2.1 & K4 (K4..M0) & $1.23(0.07 . .1 .43)$ \\
\hline $\mathrm{J} 3433264+2533319$ & $18.69 \pm 0.27$ & $17.72 \pm 0.17$ & $0.67 \pm 0.32$ & $0.72 \pm 0.23$ & $1.42 \pm 0.24$ & $1.59 \pm 0.25$ & 1.2 & K6 (K3..M0) & $1.12(0.04 . .1 .48)$ \\
\hline $\mathrm{J} 3433277+2525393$ & $23.99 \pm 7.19$ & $18.65 \pm 0.19$ & $5.18 \pm 7.20$ & $2.07 \pm 0.23$ & $2.60 \pm 0.24$ & $2.94 \pm 0.25$ & 1.9 & M6 (M5..M6) & $0.63(0.03 . .1 .27)$ \\
\hline $\mathrm{J} 3433305+2553277$ & $16.84 \pm 0.26$ & $16.35 \pm 0.17$ & $0.35 \pm 0.31$ & $0.68 \pm 0.18$ & $0.98 \pm 0.20$ & $1.11 \pm$ & 1.6 & $\mathrm{~K} 0(\mathrm{~F} 8 . . \mathrm{K} 1)$ & $0.51(0.47 . .1 .46)$ \\
\hline $\mathrm{J} 3433311+2520522$ & $16.86 \pm 0.26$ & $15.80 \pm 0.17$ & $0.73 \pm 0.31$ & $1.26 \pm 0.18$ & $1.71 \pm 0.18$ & $1.85 \pm 0.18$ & 4.5 & M0 (M0..M3) & $1.27(0.20 . .1 .41)$ \\
\hline $\mathrm{J} 3433321+2547298$ & $17.82 \pm 0.27$ & $17.36 \pm 0.17$ & $0.31 \pm 0.32$ & $0.91 \pm 0.20$ & $1.36 \pm 0.22$ & $1.72 \pm 0.24$ & 3.2 & K4 (K0..K7) & $0.59(0.30 . .1 .27)$ \\
\hline $\mathrm{J} 3433375+2539179$ & $18.30 \pm 0.27$ & $18.46 \pm 0.19$ & $-0.40 \pm 0.32$ & $1.57 \pm 0.23$ & $1.90 \pm 0.28$ & $1.51 \pm \ldots$ & 24.8 & K4 (G3..M1) & $0.92(0.36 . .1 .47)$ \\
\hline $\mathrm{J} 3433377+2537419$ & $18.98 \pm 0.27$ & $17.76 \pm 0.17$ & $0.83 \pm 0.32$ & $0.59 \pm 0.24$ & $1.47 \pm 0.23$ & $1.58 \pm$ & 1.8 & K7 (K6..M2) & $1.47(0.14 . .1 .47)$ \\
\hline $\mathrm{J} 3433390+2526118$ & $17.35 \pm 0.26$ & $16.80 \pm 0.17$ & $0.41 \pm 0.31$ & $0.95 \pm 0.18$ & $1.45 \pm 0.19$ & $1.43 \pm 0.21$ & 2.3 & $\mathrm{~K} 4$ (K0..K6) & $0.55(0.22 . .1 .22)$ \\
\hline $\mathrm{J} 3433395+2534450$ & $18.78 \pm 0.27$ & $17.42 \pm 0.17$ & $1.19 \pm 0.32$ & $1.12 \pm 0.19$ & $1.71 \pm 0.20$ & $1.96 \pm 0.22$ & 1.2 & M2 (M1..M3) & $0.63(0.20 . .1 .09)$ \\
\hline $\mathrm{J} 3433496+2533504$ & $17.54 \pm 0.26$ & $16.91 \pm 0.17$ & $0.33 \pm 0.31$ & $0.99 \pm 0.18$ & $1.43 \pm 0.19$ & $1.54 \pm 0.20$ & 2.8 & K4 (K3..M0) & $1.12(0.08 . .1 .49)$ \\
\hline $\mathrm{J} 3433507+2550134$ & $17.01 \pm 0.27$ & $15.97 \pm 0.17$ & $0.38 \pm 0.31$ & $-1.11 \pm 0.22$ & $-0.65 \pm 0.23$ & $-0.41 \pm$ & 15.8 & B0 (B0..A8) & $2.46(0.18 . .2 .46)$ \\
\hline $\mathrm{J} 3433512+2538415$ & $15.85 \pm 0.26$ & $15.24 \pm 0.17$ & $0.40 \pm 0.31$ & $0.68 \pm 0.17$ & $1.01 \pm 0.18$ & $1.04 \pm 0.18$ & 1.7 & K0 (F8..K3) & $0.76(0.10 . .1 .56)$ \\
\hline $\mathrm{J} 3433541+2550019$ & $16.63 \pm 0.26$ & $15.35 \pm 0.17$ & $0.81 \pm 0.31$ & $-0.27 \pm 0.18$ & $0.08 \pm 0.18$ & $-0.09 \pm 0.19$ & 17.5 & F0 (A3..G9) & $1.79(0.43 . .1 .79)$ \\
\hline $\mathrm{J} 3433548+2526146$ & $18.49 \pm 0.27$ & $18.01 \pm 0.17$ & $0.25 \pm 0.32$ & $0.90 \pm 0.25$ & $1.69 \pm 0.25$ & $1.52 \pm 0.35$ & 3.9 & $\mathrm{~K} 4(\mathrm{~K} 0 . . \mathrm{K} 7)$ & $0.87(0.14 . .1 .46)$ \\
\hline $\mathrm{J} 3433570+2529558$ & $16.69 \pm 0.26$ & $16.03 \pm 0.17$ & $0.29 \pm 0.31$ & $0.42 \pm 0.18$ & $0.58 \pm 0.19$ & $0.37 \pm 0.21$ & 3.2 & F5 (F5..K0) & $1.41(0.10 . .1 .41)$ \\
\hline $\mathrm{J} 3433586+2543308$ & $18.50 \pm 0.27$ & $17.78 \pm 0.17$ & $0.33 \pm 0.32$ & $0.96 \pm 0.21$ & $1.54 \pm 0.22$ & $1.46 \pm 0.26$ & 2.8 & K4 (K4..M0) & $1.49(0.13 . .1 .49)$ \\
\hline $\mathrm{J} 3433590+2536554$ & $18.84 \pm 0.27$ & $17.92 \pm 0.17$ & $0.64 \pm 0.32$ & $1.86 \pm 0.18$ & $2.30 \pm 0.19$ & $2.45 \pm 0.20$ & 25.1 & M3 (M0..M4) & $1.06(0.07 . .1 .47)$ \\
\hline $\mathrm{J} 3433595+2531435$ & $17.71 \pm 0.26$ & $17.16 \pm 0.17$ & $0.43 \pm 0.31$ & $0.89 \pm 0.19$ & $1.38 \pm 0.21$ & $1.52 \pm$ & 1.3 & $\mathrm{~K} 4$ (K1..K6) & $0.45(0.19 . .1 .27)$ \\
\hline $\mathrm{J} 3433597+2520334$ & $18.57 \pm 0.27$ & $19.13 \pm 0.36$ & $-1.08 \pm 0.45$ & $2.34 \pm 0.37$ & $2.59 \pm 0.39$ & $2.57 \pm 0.42$ & 50.5 & K4 (G1..M1) & $1.94(0.23 . .1 .94)$ \\
\hline $\mathrm{J} 3433613+2547564$ & $16.43 \pm 0.26$ & $15.75 \pm 0.17$ & $0.35 \pm 0.31$ & $0.42 \pm 0.18$ & $0.73 \pm 0.18$ & $0.76 \pm 0.19$ & 1.0 & G4 (G0..K2) & $1.22(0.20 . .1 .39)$ \\
\hline $\mathrm{J} 3433615+2549558$ & $16.49 \pm 0.26$ & $14.05 \pm 0.17$ & $2.44 \pm 0.31$ & $-2.98 \pm 0.26$ & $-2.16 \pm 0.26$ & $-1.80 \pm 0.28$ & 246.2 & B0 (B0..G8) & $0.00(0.00 . .1 .44)$ \\
\hline $\mathrm{J} 3433618+2523047$ & $17.39 \pm 0.26$ & $16.73 \pm 0.17$ & $0.35 \pm 0.31$ & $0.32 \pm 0.20$ & $0.62 \pm 0.25$ & $1.11 \pm 0.22$ & 4.1 & G6 (F5..K4) & $1.21(0.15 . .1 .45)$ \\
\hline $\mathrm{J} 3433623+2531216$ & $17.22 \pm 0.26$ & $16.68 \pm 0.17$ & $0.41 \pm 0.31$ & $0.38 \pm 0.19$ & $1.00 \pm 0.20$ & $0.91 \pm 0.24$ & 1.8 & G9 (F7..K1) & $0.49(0.10 . .1 .29)$ \\
\hline $\mathrm{J} 3433634+2544528$ & $17.24 \pm 0.26$ & $16.48 \pm 0.17$ & $0.49 \pm 0.31$ & $0.89 \pm 0.18$ & $1.41 \pm 0.18$ & $1.41 \pm 0.19$ & 1.6 & $\mathrm{~K} 4$ (K2..K6) & $1.02(0.73 . .1 .46)$ \\
\hline $\mathrm{J} 3433663+2539087$ & $18.48 \pm 0.27$ & $17.68 \pm 0.17$ & $0.44 \pm 0.32$ & $1.39 \pm 0.19$ & $1.71 \pm 0.20$ & $2.15 \pm 0.20$ & 12.2 & M0 (K7..M3) & $1.34(0.28 . .1 .40)$ \\
\hline $\mathrm{J} 3433676+2531288$ & $17.40 \pm 0.26$ & $16.93 \pm 0.17$ & $0.32 \pm 0.31$ & $0.11 \pm 0.22$ & $0.58 \pm 0.25$ & $0.77 \pm 0.30$ & 2.3 & F6 (A8..G9) & $0.55(0.03 . .1 .46)$ \\
\hline $\mathrm{J} 3433681+2537135$ & $18.21 \pm 0.27$ & $16.82 \pm 0.17$ & $1.20 \pm 0.32$ & $1.10 \pm 0.18$ & $1.81 \pm 0.18$ & $1.88 \pm 0.19$ & 1.4 & M2 (M0..M3) & $0.73(0.07 . .1 .20)$ \\
\hline $\mathrm{J} 3433685+2525175$ & $18.07 \pm 0.26$ & $17.58 \pm 0.17$ & $0.46 \pm 0.31$ & $0.94 \pm 0.21$ & $1.29 \pm 0.27$ & $1.40 \pm \ldots$ & 1.8 & K4 (G6..K4) & $0.13(0.13 . .1 .50)$ \\
\hline
\end{tabular}




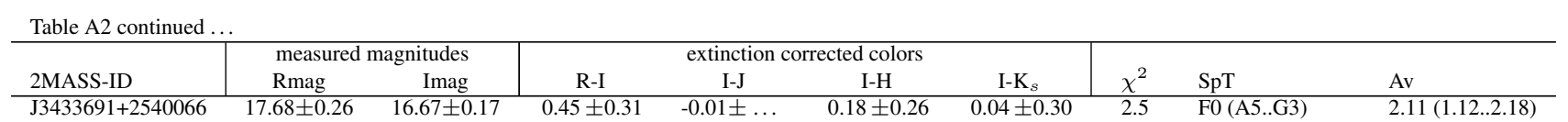


Tabelle A3 Basic data of detected objects which where not detected by the 2MASS. The table is divided by two horizontal lines in three sections. First red objects with Pleiades like $R I$ photometry are listed. These are more Pleiades BD candidates. Second bluer objects with Pleiades like Photometry are listed and afterward again objects which fall onto the Pleiades main sequence assuming that they are unresolved equal mass binaries. All classes of objects are separated by horizontal lines. Last objects with apparent $R$ and $I$ magnitudes making it unlikely for them to be members of the Pleiades. All objects listed in this table are detected on both, $R$ and $I$ band images at the same position. So (almost) all objects contained in this list are real astronomical objects from outside our solar system. Since none of these objects is listed in the $2 \mathrm{mass}$ catalog most of them are blue.

\begin{tabular}{|c|c|c|c|c|c|}
\hline$\overline{\text { Id }}$ & Imag & $\overline{R-I}$ & $\overline{\mathrm{Id}}$ & Imag & $\overline{R-I}$ \\
\hline GSHCTK J034105+253220 & $18.3 \pm 0.2$ & $2.4 \pm 0.7$ & GSHCTK J034107+254139 & $20.3 \pm 0.5$ & $3.2 \pm 5.4$ \\
\hline GSHCTK J034107+254707 & $20.3 \pm 0.5$ & $2.4 \pm 2.8$ & GSHCTK J034107+254006 & $20.1 \pm 0.4$ & $4.0 \pm 8.9$ \\
\hline GSHCTK J034108+252851 & $18.1 \pm 0.2$ & $2.4 \pm 0.5$ & GSHCTK J034109+252603 & $18.4 \pm 0.2$ & $2.2 \pm 0.5$ \\
\hline GSHCTK J034110+251957 & $19.3 \pm 0.2$ & $2.4 \pm 1.0$ & GSHCTK J034110+252158 & $18.9 \pm 0.2$ & $2.3 \pm 0.9$ \\
\hline GSHCTK J034111+253718 & $18.2 \pm 0.2$ & $2.2 \pm 0.4$ & GSHCTK J034111+253557 & $19.7 \pm 0.3$ & $2.4 \pm 1.4$ \\
\hline GSHCTK J034115+254657 & $19.6 \pm 0.3$ & $2.3 \pm 1.2$ & GSHCTK J034116+252519 & $17.8 \pm 0.2$ & $2.4 \pm 0.4$ \\
\hline GSHCTK J034116+253444 & $19.1 \pm 0.2$ & $2.4 \pm 0.8$ & GSHCTK J034116+254743 & $18.7 \pm 0.2$ & $2.7 \pm 0.8$ \\
\hline GSHCTK J034117+252526 & $17.9 \pm 0.2$ & $2.1 \pm 0.4$ & GSHCTK J034117+253309 & $19.5 \pm 0.3$ & $2.3 \pm 1.0$ \\
\hline GSHCTK J034117+253236 & $19.0 \pm 0.2$ & $2.5 \pm 0.8$ & GSHCTK J034123+253851 & $20.2 \pm 0.4$ & $2.5 \pm 2.5$ \\
\hline GSHCTK J034123+253827 & $18.8 \pm 0.2$ & $2.7 \pm 0.8$ & GSHCTK J034124+252849 & $19.6 \pm 0.3$ & $2.4 \pm 1.3$ \\
\hline GSHCTK J034124+253951 & $17.5 \pm 0.2$ & $2.2 \pm 0.3$ & GSHCTK J034127+252329 & $18.0 \pm 0.2$ & $2.1 \pm 0.4$ \\
\hline GSHCTK J034127+252349 & $17.5 \pm 0.2$ & $2.4 \pm 0.4$ & GSHCTK J034133+254256 & $19.6 \pm 0.3$ & $2.8 \pm 1.7$ \\
\hline GSHCTK J034136+254135 & $19.6 \pm 0.3$ & $3.2 \pm 2.1$ & GSHCTK J034138+254825 & $19.8 \pm 0.3$ & $3.9 \pm 6.7$ \\
\hline GSHCTK J034138+255126 & $19.0 \pm 0.2$ & $2.4 \pm 0.9$ & GSHCTK J034142+253451 & $19.1 \pm 0.2$ & $2.7 \pm 1.0$ \\
\hline GSHCTK J034142+254128 & $19.4 \pm 0.3$ & $3.7 \pm 3.3$ & GSHCTK J034144+254622 & $20.6 \pm 0.6$ & $2.3 \pm 3.0$ \\
\hline GSHCTK J034145+253132 & $19.9 \pm 0.3$ & $3.4 \pm 3.6$ & GSHCTK J034149+254259 & $19.4 \pm 0.2$ & $3.3 \pm 2.5$ \\
\hline GSHCTK J034150+255060 & $18.2 \pm 0.2$ & $2.6 \pm 0.5$ & GSHCTK J034152+253745 & $18.9 \pm 0.2$ & $2.2 \pm 0.6$ \\
\hline GSHCTK J034153+254803 & $19.0 \pm 0.2$ & $2.8 \pm 1.1$ & GSHCTK J034155+252254 & $19.5 \pm 0.3$ & $3.1 \pm 2.9$ \\
\hline GSHCTK J034200+252152 & $19.4 \pm 0.3$ & $3.4 \pm 2.5$ & GSHCTK J034202+253044 & $19.5 \pm 0.3$ & $3.0 \pm 1.7$ \\
\hline GSHCTK J034202+255312 & $18.6 \pm 0.2$ & $2.5 \pm 0.5$ & GSHCTK J034205+253451 & $19.4 \pm 0.2$ & $3.0 \pm 1.5$ \\
\hline GSHCTK J034205+252252 & $18.0 \pm 0.2$ & $2.3 \pm 0.4$ & GSHCTK J034207+252805 & $17.2 \pm 0.2$ & $2.2 \pm 0.3$ \\
\hline GSHCTK J034208+253943 & $19.6 \pm 0.2$ & $3.2 \pm 2.6$ & GSHCTK J034209+252916 & $18.8 \pm 0.2$ & $2.7 \pm 0.8$ \\
\hline GSHCTK J034211+252438 & $19.2 \pm 0.2$ & $3.5 \pm 2.2$ & GSHCTK J034212+252548 & $19.9 \pm 0.3$ & $2.5 \pm 1.6$ \\
\hline GSHCTK J034212+253026 & $18.9 \pm 0.2$ & $2.5 \pm 0.8$ & GSHCTK J034213+255306 & $18.1 \pm 0.2$ & $2.6 \pm 0.5$ \\
\hline GSHCTK J034218+253704 & $18.7 \pm 0.2$ & $2.5 \pm 0.6$ & GSHCTK J034223+252050 & $17.4 \pm 0.2$ & $2.1 \pm 0.3$ \\
\hline GSHCTK J034223+255045 & $17.8 \pm 0.2$ & $2.2 \pm 0.4$ & GSHCTK J034224+254912 & $19.1 \pm 0.2$ & $3.0 \pm 1.2$ \\
\hline GSHCTK J034225+254837 & $18.8 \pm 0.2$ & $2.9 \pm 0.9$ & GSHCTK J034226+253925 & $18.1 \pm 0.2$ & $2.3 \pm 0.4$ \\
\hline GSHCTK J034226+253923 & $18.3 \pm 0.2$ & $2.6 \pm 0.5$ & GSHCTK J034229+252712 & $19.0 \pm 0.2$ & $2.7 \pm 0.9$ \\
\hline GSHCTK J034231+252714 & $20.2 \pm 0.4$ & $2.8 \pm 2.8$ & GSHCTK J034234+253832 & $19.1 \pm 0.2$ & $2.2 \pm 0.7$ \\
\hline GSHCTK J034236+255014 & $18.3 \pm 0.2$ & $2.3 \pm 0.5$ & GSHCTK J034237+255328 & $19.8 \pm 0.3$ & $4.0 \pm 5.6$ \\
\hline GSHCTK J034239+252145 & $18.8 \pm 0.2$ & $3.0 \pm 1.0$ & GSHCTK J034240+253405 & $20.7 \pm 0.7$ & $2.6 \pm 3.6$ \\
\hline GSHCTK J034240+255124 & $19.0 \pm 0.2$ & $2.9 \pm 1.1$ & GSHCTK J034243+253759 & $18.9 \pm 0.2$ & $2.4 \pm 0.6$ \\
\hline GSHCTK J034244+253028 & $19.5 \pm 0.3$ & $2.6 \pm 1.3$ & GSHCTK J034245+252449 & $18.5 \pm 0.2$ & $2.5 \pm 0.5$ \\
\hline GSHCTK J034247+254509 & $19.5 \pm 0.3$ & $3.1 \pm 2.4$ & GSHCTK J034248+253657 & $18.8 \pm 0.2$ & $2.9 \pm 0.9$ \\
\hline GSHCTK J034248+255151 & $19.0 \pm 0.2$ & $2.7 \pm 1.3$ & GSHCTK J034251+253318 & $18.0 \pm 0.2$ & $2.5 \pm 0.4$ \\
\hline GSHCTK J034251+254903 & $19.9 \pm 0.4$ & $2.9 \pm 2.8$ & GSHCTK J034254+252437 & $19.5 \pm 0.3$ & $2.6 \pm 1.3$ \\
\hline GSHCTK J034255+254310 & $19.6 \pm 0.3$ & $3.0 \pm 2.6$ & GSHCTK J034257+253703 & $18.6 \pm 0.2$ & $2.3 \pm 0.5$ \\
\hline GSHCTK J034259+253358 & $17.7 \pm 0.2$ & $2.3 \pm 0.4$ & GSHCTK J034260+254537 & $18.4 \pm 0.2$ & $2.4 \pm 0.5$ \\
\hline GSHCTK J034301+255120 & $18.7 \pm 0.2$ & $2.4 \pm 0.6$ & GSHCTK J034301+254756 & $19.3 \pm 0.2$ & $3.1 \pm 1.8$ \\
\hline GSHCTK J034302+253457 & $18.1 \pm 0.2$ & $2.3 \pm 0.4$ & GSHCTK J034304+253738 & $19.9 \pm 0.3$ & $4.7 \pm 10.7$ \\
\hline GSHCTK J034308+252353 & $18.5 \pm 0.2$ & $2.2 \pm 0.5$ & GSHCTK J034311+252700 & $18.3 \pm 0.2$ & $2.4 \pm 0.5$ \\
\hline GSHCTK J034312+254440 & $18.6 \pm 0.2$ & $2.5 \pm 0.6$ & GSHCTK J034316+254630 & $18.2 \pm 0.2$ & $2.2 \pm 0.4$ \\
\hline GSHCTK J034317+252041 & $18.0 \pm 0.2$ & $2.4 \pm 0.4$ & GSHCTK J034329+254851 & $17.8 \pm 0.2$ & $2.0 \pm 0.4$ \\
\hline GSHCTK J034330+254003 & $18.1 \pm 0.2$ & $2.3 \pm 0.4$ & & \pm & \\
\hline GSHCTK J034160+252435 & $16.0 \pm 0.2$ & $1.7 \pm 0.3$ & GSHCTK J034217+252212 & $16.2 \pm 0.2$ & $1.6 \pm 0.3$ \\
\hline GSHCTK J034320+253747 & $15.0 \pm 0.2$ & $1.7 \pm 0.3$ & GSHCTK J034322+251932 & $16.7 \pm 0.2$ & $1.6 \pm 0.3$ \\
\hline GSHCTK J034139+254936 & $18.5 \pm 0.2$ & $3.2 \pm 1.0$ & GSHCTK J034152+255323 & $18.6 \pm 0.2$ & $3.1 \pm 0.7$ \\
\hline GSHCTK J034059+254634 & $18.6 \pm 0.2$ & $0.7 \pm 0.4$ & GSHCTK J034059+254050 & $21.0 \pm 0.9$ & $-1.1 \pm 1.0$ \\
\hline GSHCTK J034060+254450 & $19.9 \pm 0.4$ & $-1.0 \pm 0.5$ & GSHCTK J034060+251917 & $19.6 \pm 0.4$ & $-1.0 \pm 0.5$ \\
\hline GSHCTK J034100+254433 & $19.2 \pm 0.2$ & $0.0 \pm 0.4$ & GSHCTK J034100+254258 & $17.2 \pm 0.2$ & $0.9 \pm 0.3$ \\
\hline GSHCTK J034101+253902 & $21.2 \pm 1.1$ & $-1.9 \pm 1.1$ & GSHCTK J034100+252335 & $17.7 \pm 0.2$ & $0.1 \pm 0.3$ \\
\hline GSHCTK J034101+254453 & $20.1 \pm 0.4$ & $-1.1 \pm 0.5$ & GSHCTK J034101+254209 & $21.9 \pm 2.6$ & $-2.3 \pm 2.6$ \\
\hline GSHCTK J034101+253315 & $19.6 \pm 0.3$ & $-1.0 \pm 0.4$ & GSHCTK J034101+254238 & $18.5 \pm 0.2$ & $0.7 \pm 0.4$ \\
\hline GSHCTK J034102+254601 & $18.9 \pm 0.2$ & $-0.3 \pm 0.3$ & GSHCTK J034102+252534 & $20.6 \pm 0.2$ & $0.4 \pm 0.4$ \\
\hline GSHCTK J034102+252335 & $20.6 \pm 0.2$ & $-0.9 \pm 0.4$ & GSHCTK J034102+251903 & $21.2 \pm 0.4$ & $-0.9 \pm 0.5$ \\
\hline GSHCTK J034102+253309 & $20.2 \pm 0.2$ & $-1.1 \pm 0.3$ & GSHCTK J034102+254245 & $18.6 \pm 0.2$ & $0.4 \pm 0.3$ \\
\hline GSHCTK J034102+253112 & $19.8 \pm 0.3$ & $-1.3 \pm 0.4$ & GSHCTK J034102+254530 & $19.1 \pm 0.2$ & $0.6 \pm 0.4$ \\
\hline GSHCTK J034102+254152 & $18.7 \pm 0.2$ & $0.8 \pm 0.4$ & GSHCTK J034103+254316 & $18.6 \pm 0.2$ & $0.9 \pm 0.4$ \\
\hline GSHCTK J034103+253503 & $18.2 \pm 0.2$ & $0.5 \pm 0.3$ & GSHCTK J034103+251943 & $18.2 \pm 0.2$ & $1.0 \pm 0.4$ \\
\hline GSHCTK J034103+254725 & $18.4 \pm 0.2$ & $0.6 \pm 0.3$ & GSHCTK J034103+255358 & $19.1 \pm 0.2$ & $-0.4 \pm 0.3$ \\
\hline GSHCTK J034103+252649 & $17.7 \pm 0.2$ & $-0.1 \pm 0.3$ & GSHCTK J034103+255048 & $19.3 \pm 0.3$ & $0.6 \pm 0.4$ \\
\hline GSHCTK J034104+254933 & $20.7 \pm 0.8$ & $-0.0 \pm 1.0$ & GSHCTK J034104+254422 & $18.8 \pm 0.2$ & $1.5 \pm 0.6$ \\
\hline GSHCTK J034104+252352 & $17.7 \pm 0.2$ & $0.7 \pm 0.3$ & GSHCTK J034104+252023 & $16.8 \pm 0.2$ & $0.8 \pm 0.3$ \\
\hline GSHCTK J034104+254734 & $18.7 \pm 0.2$ & $0.8 \pm 0.4$ & GSHCTK J034104+252813 & $18.8 \pm 0.2$ & $0.5 \pm 0.4$ \\
\hline GSHCTK J034104+254216 & $20.1 \pm 0.5$ & $0.1 \pm 0.7$ & GSHCTK J034105+255057 & $19.3 \pm 0.2$ & $1.1 \pm 0.5$ \\
\hline GSHCTK J034105+254459 & $19.5 \pm 0.3$ & $1.1 \pm 0.7$ & GSHCTK J034105+255243 & $18.5 \pm 0.2$ & $1.3 \pm 0.3$ \\
\hline GSHCTK J034106+252434 & $18.5 \pm 0.2$ & $-0.1 \pm 0.3$ & GSHCTK J034106+255400 & $23.0 \pm 1.4$ & $-4.2 \pm 1.4$ \\
\hline GSHCTK J034106+253738 & $19.5 \pm 0.3$ & $1.7 \pm 0.7$ & GSHCTK J034107+252857 & $17.8 \pm 0.2$ & $1.4 \pm 0.3$ \\
\hline GSHCTK J034107+254623 & $18.1 \pm 0.2$ & $0.7 \pm 0.3$ & GSHCTK J034107+255405 & $18.4 \pm 0.2$ & $1.4 \pm 0.3$ \\
\hline GSHCTK J034107+253853 & $17.8 \pm 0.2$ & $1.0 \pm 0.3$ & GSHCTK J034108+253543 & $20.1 \pm 0.5$ & $1.7 \pm 1.2$ \\
\hline GSHCTK J034108+253846 & $18.0 \pm 0.2$ & $0.9 \pm 0.3$ & GSHCTK J034108+254442 & $18.7 \pm 0.2$ & $0.8 \pm 0.3$ \\
\hline GSHCTK J034108+255400 & $18.2 \pm 0.2$ & $0.6 \pm 0.3$ & GSHCTK J034108+253623 & $19.0 \pm 0.2$ & $0.7 \pm 0.4$ \\
\hline GSHCTK J034109+254254 & $17.9 \pm 0.2$ & $1.1 \pm 0.3$ & GSHCTK J034109+252906 & $19.3 \pm 0.2$ & $0.9 \pm 0.4$ \\
\hline GSHCTK J034109+252435 & $17.7 \pm 0.2$ & $1.2 \pm 0.3$ & GSHCTK J034109+253727 & $20.4 \pm 0.5$ & $-0.1 \pm 0.6$ \\
\hline GSHCTK J034109+253227 & $19.2 \pm 0.2$ & $1.1 \pm 0.4$ & GSHCTK J034109+252829 & $19.4 \pm 0.3$ & $2.0 \pm 0.8$ \\
\hline GSHCTK J034109+254008 & $20.3 \pm 0.4$ & $-0.2 \pm 0.6$ & GSHCTK J034110+252211 & $17.9 \pm 0.2$ & $1.2 \pm 0.3$ \\
\hline GSHCTK J034109+253253 & $18.8 \pm 0.2$ & $0.8 \pm 0.4$ & GSHCTK J034110+252926 & $19.1 \pm 0.2$ & $0.3 \pm 0.4$ \\
\hline
\end{tabular}




\begin{tabular}{|c|c|c|c|c|c|}
\hline Id & Imag & $R-I$ & Id & Imag & $R-I$ \\
\hline GSHCTK J034110+254249 & $19.8 \pm 0.3$ & $-0.5 \pm 0.4$ & GSHCTK J034110+255013 & $18.8 \pm 0.2$ & $1.5 \pm 0.5$ \\
\hline GSHCTK J034110+254752 & $19.9 \pm 0.4$ & $0.0 \pm 0.5$ & GSHCTK J034110+253253 & $18.7 \pm 0.2$ & $0.7 \pm 0.3$ \\
\hline GSHCTK J034110+254026 & $20.2 \pm 0.4$ & $0.1 \pm 0.6$ & GSHCTK J034111+253435 & $19.4 \pm 0.2$ & $0.9 \pm 0.4$ \\
\hline GSHCTK J034111+252200 & $19.1 \pm 0.2$ & $1.0 \pm 0.5$ & GSHCTK J034111+254836 & $18.7 \pm 0.2$ & $1.8 \pm 0.5$ \\
\hline GSHCTK J034111+254407 & $19.5 \pm 0.3$ & $0.2 \pm 0.4$ & GSHCTK J034111+255258 & $18.2 \pm 0.2$ & $0.7 \pm 0.3$ \\
\hline GSHCTK J034111+255250 & $18.7 \pm 0.2$ & $0.4 \pm 0.3$ & GSHCTK J034111+255154 & $16.1 \pm 0.2$ & $0.8 \pm 0.3$ \\
\hline GSHCTK J034111+255017 & $22.3 \pm 3.0$ & $-1.6 \pm 3.0$ & GSHCTK J034111+252855 & $20.6 \pm 0.6$ & $-0.6 \pm 0.7$ \\
\hline GSHCTK J034111+253256 & $19.1 \pm 0.2$ & $0.6 \pm 0.4$ & GSHCTK J034111+254825 & $19.1 \pm 0.2$ & $1.1 \pm 0.4$ \\
\hline GSHCTK J034111+253621 & $18.3 \pm 0.2$ & $0.8 \pm 0.3$ & GSHCTK J034112+253419 & $19.5 \pm 0.3$ & $0.6 \pm 0.4$ \\
\hline GSHCTK J034112+253001 & $17.9 \pm 0.2$ & $0.5 \pm 0.3$ & GSHCTK J034112+254404 & $17.9 \pm 0.2$ & $0.6 \pm 0.3$ \\
\hline GSHCTK J034112+255203 & $19.1 \pm 0.2$ & $0.7 \pm 0.4$ & GSHCTK J034112+254648 & $17.9 \pm 0.2$ & $0.8 \pm 0.3$ \\
\hline GSHCTK J034112+254351 & $18.2 \pm 0.2$ & $0.6 \pm 0.3$ & GSHCTK J034112+254236 & $19.1 \pm 0.2$ & $0.5 \pm 0.4$ \\
\hline GSHCTK J034113+253141 & $17.6 \pm 0.2$ & $0.5 \pm 0.3$ & GSHCTK J034112+254145 & $17.1 \pm 0.2$ & $0.9 \pm 0.3$ \\
\hline GSHCTK J034113+252450 & $18.6 \pm 0.2$ & $1.1 \pm 0.4$ & GSHCTK J034113+252351 & $17.6 \pm 0.2$ & $1.2 \pm 0.3$ \\
\hline GSHCTK J034113+253913 & $20.0 \pm 0.3$ & $1.6 \pm 0.9$ & GSHCTK J034113+254626 & $18.9 \pm 0.2$ & $1.0 \pm 0.4$ \\
\hline GSHCTK J034113+252824 & $18.8 \pm 0.2$ & $0.5 \pm 0.3$ & GSHCTK J034113+254145 & $20.1 \pm 0.3$ & $-0.4 \pm 0.4$ \\
\hline GSHCTK J034113+254019 & $19.3 \pm 0.2$ & $0.9 \pm 0.4$ & GSHCTK J034113+252430 & $21.1 \pm 1.3$ & $-1.8 \pm 1.3$ \\
\hline GSHCTK J034114+252930 & $19.0 \pm 0.2$ & $0.9 \pm 0.4$ & GSHCTK J034114+254619 & $18.9 \pm 0.2$ & $0.3 \pm 0.4$ \\
\hline GSHCTK J034114+254533 & $21.0 \pm 0.9$ & $-1.0 \pm 1.0$ & GSHCTK J034114+253203 & $19.3 \pm 0.2$ & $1.1 \pm 0.4$ \\
\hline GSHCTK J034114+253829 & $21.5 \pm 1.2$ & $0.0 \pm 1.4$ & GSHCTK J034114+253132 & $18.7 \pm 0.2$ & $0.9 \pm 0.3$ \\
\hline GSHCTK J034114+254420 & $19.6 \pm 0.3$ & $0.7 \pm 0.5$ & GSHCTK J034115+253909 & $18.3 \pm 0.2$ & $0.3 \pm 0.3$ \\
\hline GSHCTK J034115+254154 & $20.2 \pm 0.4$ & $-0.1 \pm 0.6$ & GSHCTK J034115+253455 & $20.9 \pm 0.7$ & $0.9 \pm 1.3$ \\
\hline GSHCTK J034115+254349 & $18.0 \pm 0.2$ & $0.5 \pm 0.3$ & GSHCTK J034115+253811 & $21.1 \pm 0.9$ & $-1.1 \pm 1.0$ \\
\hline GSHCTK J034115+252842 & $16.7 \pm 0.2$ & $0.7 \pm 0.3$ & GSHCTK J034115+255009 & $18.8 \pm 0.2$ & $1.0 \pm 0.4$ \\
\hline GSHCTK J034115+253119 & $18.8 \pm 0.2$ & $1.8 \pm 0.4$ & GSHCTK J034116+253004 & $18.4 \pm 0.2$ & $0.9 \pm 0.3$ \\
\hline GSHCTK J034116+254705 & $18.3 \pm 0.2$ & $3.4 \pm 1.0$ & GSHCTK J034116+253738 & $19.4 \pm 0.3$ & $0.3 \pm 0.4$ \\
\hline GSHCTK J034116+251917 & $21.8 \pm 1.7$ & $-2.0 \pm 1.7$ & GSHCTK J034116+253214 & $19.4 \pm 0.2$ & $1.3 \pm 0.5$ \\
\hline GSHCTK J034116+252103 & $20.4 \pm 0.5$ & $-0.6 \pm 0.6$ & GSHCTK J034116+254342 & $18.2 \pm 0.2$ & $1.1 \pm 0.3$ \\
\hline GSHCTK J034117+252205 & $17.5 \pm 0.2$ & $0.0 \pm 0.3$ & GSHCTK J034117+254659 & $18.1 \pm 0.2$ & $=0.3$ \\
\hline GSHCTK J034117+254905 & $17.8 \pm 0.2$ & $0.9 \pm 0.3$ & GSHCTK J034117+253050 & $19.0 \pm 0.2$ & $1.2 \pm 0.4$ \\
\hline GSHCTK J034117+253915 & $19.0 \pm 0.2$ & $0.7 \pm 0.4$ & GSHCTK J034117+251943 & $21.5 \pm 1.3$ & $-1.9 \pm 1.3$ \\
\hline GSHCTK J034117+252437 & $21.4 \pm 1.6$ & $-2.8 \pm 1.6$ & GSHCTK J034118+252919 & $19.6 \pm 0.3$ & $0.7 \pm 0.5$ \\
\hline GSHCTK J034117+254342 & $19.1 \pm 0.2$ & $1.2 \pm 0.4$ & GSHCTK J034118+252428 & $19.3 \pm 0.3$ & $-1.0 \pm 0.4$ \\
\hline GSHCTK J034118+254520 & $18.2 \pm 0.2$ & $1.9 \pm 0.4$ & GSHCTK J034118+252658 & $16.8 \pm 0.2$ & $-0.2 \pm 0.3$ \\
\hline GSHCTK J034118+252344 & $19.0 \pm 0.2$ & $0.2 \pm 0.4$ & GSHCTK J034118+254836 & $19.0 \pm 0.2$ & $0.8 \pm 0.4$ \\
\hline GSHCTK J034119+253508 & $18.2 \pm 0.2$ & $0.6 \pm 0.3$ & GSHCTK J034119+255310 & $23.7 \pm 9.2$ & $-4.3 \pm 9.2$ \\
\hline GSHCTK J034119+254325 & $19.1 \pm 0.2$ & $0.9 \pm 0.4$ & GSHCTK J034119+253043 & $18.8 \pm 0.2$ & $1.6 \pm 0.5$ \\
\hline GSHCTK J034119+253021 & $19.1 \pm 0.2$ & $0.5 \pm 0.4$ & GSHCTK J034119+254509 & $19.0 \pm 0.2$ & $1.0 \pm 0.4$ \\
\hline GSHCTK J034119+254411 & $19.3 \pm 0.3$ & $1.7 \pm 0.6$ & GSHCTK J034119+253847 & $18.2 \pm 0.2$ & $1.3 \pm 0.3$ \\
\hline GSHCTK J034119+253320 & $19.8 \pm 0.3$ & $-0.7 \pm 0.4$ & GSHCTK J034119+254440 & $18.4 \pm 0.2$ & $0.7 \pm 0.3$ \\
\hline GSHCTK J034120+253953 & $18.6 \pm 0.2$ & $0.7 \pm 0.3$ & GSHCTK J034120+255110 & $18.8 \pm 0.2$ & $0.9 \pm 0.4$ \\
\hline GSHCTK J034120+254555 & $19.1 \pm 0.2$ & $1.0 \pm 0.4$ & GSHCTK J034120+254845 & $18.9 \pm 0.2$ & $1.5 \pm 0.5$ \\
\hline GSHCTK J034120+253404 & $19.3 \pm 0.2$ & $0.2 \pm 0.4$ & GSHCTK J034120+253214 & $17.7 \pm 0.2$ & $0.9 \pm 0.3$ \\
\hline GSHCTK J034120+254121 & $15.6 \pm 0.2$ & $0.7 \pm 0.3$ & GSHCTK J034120+255237 & $19.5 \pm 0.3$ & $0.3 \pm 0.4$ \\
\hline GSHCTK J034121+255040 & $20.2 \pm 0.6$ & $-0.4 \pm 0.7$ & GSHCTK J034121+254245 & $19.2 \pm 0.2$ & $0.3 \pm 0.4$ \\
\hline GSHCTK J034121+253730 & $20.9 \pm 0.7$ & $-1.1 \pm 0.7$ & GSHCTK J034121+253601 & $19.5 \pm 0.3$ & $0.4 \pm 0.4$ \\
\hline GSHCTK J034121+253247 & $18.6 \pm 0.2$ & $1.9 \pm 0.4$ & GSHCTK J034122+254101 & $19.9 \pm 0.3$ & $0.6 \pm 0.5$ \\
\hline GSHCTK J034122+254905 & $18.1 \pm 0.2$ & $1.4 \pm 0.3$ & GSHCTK J034122+254617 & $18.0 \pm 0.2$ & $0.4 \pm 0.3$ \\
\hline GSHCTK J034122+254137 & $22.9 \pm 4.0$ & $-3.1 \pm 4.0$ & GSHCTK J034122+255201 & $19.7 \pm 0.4$ & $0.1 \pm 0.5$ \\
\hline GSHCTK J034122+252855 & $18.6 \pm 0.2$ & $0.9 \pm 0.3$ & GSHCTK J034123+254736 & $19.7 \pm 0.3$ & $0.6 \pm 0.5$ \\
\hline GSHCTK J034123+254427 & $17.8 \pm 0.2$ & $1.7 \pm 0.3$ & GSHCTK J034123+252333 & $21.6 \pm 1.8$ & $0.8 \pm 2.9$ \\
\hline GSHCTK J034123+254938 & $18.9 \pm 0.2$ & $0.7 \pm 0.4$ & GSHCTK J034123+252209 & $18.9 \pm 0.2$ & $0.9 \pm 0.4$ \\
\hline GSHCTK J034123+252738 & $19.0 \pm 0.2$ & $2.1 \pm 0.7$ & GSHCTK J034123+254506 & $18.4 \pm 0.2$ & $1.3 \pm 0.4$ \\
\hline GSHCTK J034123+252116 & $17.9 \pm 0.2$ & $0.2 \pm 0.3$ & GSHCTK J034124+253201 & $21.7 \pm 1.6$ & $-1.8 \pm 1.7$ \\
\hline GSHCTK J034123+252926 & $19.0 \pm 0.2$ & $1.1 \pm 0.4$ & GSHCTK J034124+252913 & $19.5 \pm 0.3$ & $0.3 \pm 0.4$ \\
\hline GSHCTK J034124+254022 & $17.4 \pm 0.2$ & $1.1 \pm 0.3$ & GSHCTK J034124+254608 & $19.5 \pm 0.3$ & $1.1 \pm 0.5$ \\
\hline GSHCTK J034125+254159 & $18.4 \pm 0.2$ & $0.9 \pm 0.3$ & GSHCTK J034125+253905 & $19.1 \pm 0.2$ & $0.9 \pm 0.4$ \\
\hline GSHCTK J034125+255237 & $18.8 \pm 0.2$ & $1.9 \pm 0.4$ & GSHCTK J034125+254511 & $20.2 \pm 0.4$ & $1.0 \pm 0.8$ \\
\hline GSHCTK J034125+252836 & $20.0 \pm 0.4$ & $0.5 \pm 0.6$ & GSHCTK J034125+252546 & $18.2 \pm 0.2$ & $1.4 \pm 0.3$ \\
\hline GSHCTK J034125+254641 & $19.5 \pm 0.3$ & $0.9 \pm 0.4$ & GSHCTK J034125+254533 & $18.7 \pm 0.2$ & $1.2 \pm 0.4$ \\
\hline GSHCTK J034126+254135 & $18.8 \pm 0.2$ & $1.3 \pm 0.4$ & GSHCTK J034126+253754 & $18.7 \pm 0.2$ & $0.2 \pm 0.3$ \\
\hline GSHCTK J034126+253128 & $19.3 \pm 0.3$ & $1.3 \pm 0.5$ & GSHCTK J034126+252320 & $20.1 \pm 0.4$ & $0.6 \pm 0.6$ \\
\hline GSHCTK J034126+253559 & $19.0 \pm 0.2$ & $0.4 \pm 0.4$ & GSHCTK J034126+253340 & $19.7 \pm 0.3$ & $2.3 \pm 1.3$ \\
\hline GSHCTK J034126+255122 & $17.3 \pm 0.2$ & $1.0 \pm 0.3$ & GSHCTK J034126+254721 & $20.7 \pm 0.6$ & $-0.8 \pm 0.7$ \\
\hline GSHCTK J034126+252504 & $18.6 \pm 0.2$ & $1.8 \pm 0.4$ & GSHCTK J034126+252305 & $18.5 \pm 0.2$ & $0.7 \pm 0.3$ \\
\hline GSHCTK J034126+253303 & $19.5 \pm 0.2$ & $-0.1 \pm 0.4$ & GSHCTK J034126+255257 & $17.3 \pm 0.2$ & $0.8 \pm 0.3$ \\
\hline GSHCTK J034127+253119 & $18.9 \pm 0.2$ & $1.9 \pm 0.5$ & GSHCTK J034127+253059 & $18.1 \pm 0.2$ & $1.2 \pm 0.3$ \\
\hline GSHCTK J034127+253736 & $18.3 \pm 0.2$ & $1.7 \pm 0.4$ & GSHCTK J034127+252333 & $17.6 \pm 0.2$ & $1.7 \pm 0.3$ \\
\hline GSHCTK J034127+254022 & $20.6 \pm 0.6$ & $-0.2 \pm 0.7$ & GSHCTK J034128+254845 & $18.9 \pm 0.2$ & $0.6 \pm 0.3$ \\
\hline GSHCTK J034128+252119 & $18.5 \pm 0.2$ & $0.4 \pm 0.3$ & GSHCTK J034128+254057 & $21.1 \pm 0.9$ & $-1.4 \pm 1.0$ \\
\hline GSHCTK J034128+254042 & $17.1 \pm 0.2$ & $0.9 \pm 0.3$ & GSHCTK J034128+253141 & $20.3 \pm 0.5$ & $-0.5 \pm 0.6$ \\
\hline GSHCTK J034129+254903 & $19.0 \pm 0.2$ & $1.0 \pm 0.4$ & GSHCTK J034129+253734 & $17.7 \pm 0.2$ & $0.9 \pm 0.3$ \\
\hline GSHCTK J034129+255241 & $18.0 \pm 0.2$ & & GSHCTK J034129+253922 & $19.7 \pm 0.3$ & \\
\hline GSHCTK J034129+254728 & $19.1 \pm 0.2$ & $1.3 \pm 0.4$ & GSHCTK J034129+251924 & $20.0 \pm 0.4$ & $-1.1 \pm 0.5$ \\
\hline GSHCTK J034130+252632 & $17.7 \pm 0.2$ & $1.5 \pm 0.3$ & GSHCTK J034130+254639 & $19.3 \pm 0.2$ & \\
\hline GSHCTK J034130+253648 & $20.0 \pm 0.4$ & $-0.3 \pm 0.5$ & GSHCTK J034130+254210 & $19.1 \pm 0.2$ & $1.6 \pm 0.5$ \\
\hline GSHCTK J034130+254726 & $18.9 \pm 0.2$ & $0.6 \pm 0.4$ & GSHCTK J034130+252130 & $20.2 \pm 0.7$ & \\
\hline GSHCTK J034130+253659 & $19.5 \pm 0.3$ & $0.1 \pm 0.4$ & GSHCTK J034130+254042 & $19.1 \pm 0.2$ & $1.2 \pm 0.4$ \\
\hline GSHCTK J034131+254637 & $19.1 \pm 0.2$ & $1.0 \pm 0.4$ & GSHCTK J034131+254236 & $20.7 \pm 0.7$ & \\
\hline GSHCTK J034131+254617 & $23.4 \pm 6.7$ & $-3.7 \pm 6.7$ & GSHCTK J034131+254121 & $20.8 \pm 0.6$ & $-0.8 \pm 0.7$ \\
\hline GSHCTK J034131+254655 & $18.4 \pm 0.2$ & $1.5 \pm 0.4$ & GSHCTK J034131+253232 & $19.1 \pm 0.2$ & $1.5 \pm 0.5$ \\
\hline GSHCTK J034131+255317 & $19.9 \pm 0.3$ & $-0.7 \pm 0.4$ & GSHCTK J034131+252820 & $18.1 \pm 0.2$ & 0.6 \\
\hline GSHCTK J034132+253847 & $20.8 \pm 0.6$ & $-0.5 \pm 0.7$ & GSHCTK J034132+253115 & $19.3 \pm 0.2$ & $1.2 \pm 0.5$ \\
\hline
\end{tabular}




\begin{tabular}{|c|c|c|c|c|c|}
\hline & Imag & $R-I$ & Id & Imag & $R-I$ \\
\hline GSHCTK J034132+252740 & $18.2 \pm 0.2$ & $1.7 \pm 0.4$ & GSHCTK J034132+254325 & $18.7 \pm 0.2$ & $2.1 \pm 0$ \\
\hline GSHCTK J034132+253342 & $18.2 \pm 0.2$ & $0.9 \pm 0.3$ & GSHCTK J034132+253019 & $19.4 \pm 0.3$ & $1.2 \pm 0.5$ \\
\hline GSHCTK J034132+254358 & $19.6 \pm 0.3$ & $0.5 \pm 0.4$ & GSHCTK J034132+254854 & $18.9 \pm 0.2$ & \\
\hline GSHCTK J034132+252404 & $17.8 \pm 0.2$ & $0.7 \pm 0.3$ & GSHCTK J034132+254440 & $19.0 \pm 0.2$ & \pm 0.4 \\
\hline GSHCTK J034132+253927 & $19.9 \pm 0.3$ & $0.8 \pm 0.5$ & $034132+254$ & & \\
\hline GSHCTK J034133+254146 & $17.9 \pm 0.2$ & $0.7 \pm 0.3$ & GSHCTK J034133+254110 & $22.4 \pm 2.6$ & $9 \pm 2.6$ \\
\hline GSHCTK J034133+253154 & $18.9 \pm 0.2$ & $0.6 \pm 0.4$ & & $20.1 \pm$ & \\
\hline GSHCTK J034133+254159 & $17.7 \pm 0.2$ & $0.9 \pm 0.3$ & GSHCTK J034133+252718 & $20.4 \pm 0.7$ & 0.7 \\
\hline GSHCTK J034133+254029 & & & & & \\
\hline GSHCTK J034134+255228 & $17.7 \pm 0.2$ & $0.8 \pm 0.3$ & GSHCTK J034134+254031 & $19.4 \pm 0.2$ & \pm \\
\hline GSHCTK J034134+253955 & $19.9 \pm 0.3$ & $0.0 \pm 0.4$ & & & \\
\hline GSHCTK J034134+253254 & $19.0 \pm 0.2$ & $0.6 \pm 0.4$ & GSHCTK & $18.0 \pm 0.2$ & .3 \\
\hline GSHCTK J034134+252716 & & $0.9 \pm 0.4$ & & & \\
\hline GSHCTK J & $18.8 \pm 0.2$ & $1.2 \pm 0.4$ & $5+254004$ & $21.1 \pm$ & $6 \pm 0.8$ \\
\hline GSHCTK J034135+253446 & $20.2 \pm 0.4$ & $-0.3 \pm 0.5$ & & & \\
\hline GSHCTK & $18.9 \pm 0.2$ & $0.5 \pm 0.4$ & & & $08+5$ \\
\hline GSHCTK J034136+253526 & $18.6 \pm 0.2$ & $1.1 \pm 0.4$ & & $19.0 \pm$ & \\
\hline GSHCTK & \pm 0.2 & $1.8 \pm 0.3$ & 26 & & .8 \\
\hline GSHCTK J034136+255237 & $20.3 \pm 0.5$ & $1.5 \pm 1.1$ & & 0.2 & $6 \pm 0.3$ \\
\hline GSHC & \pm & $1.4 \pm 0.6$ & & & $9 \pm 0.3$ \\
\hline GSH & & \pm 0.5 & & & \\
\hline GSH & & $1.8 \pm 0.7$ & & & \\
\hline GSH & & $0.5 \pm 0.4$ & & & 0.3 \\
\hline & & $-0.1 \pm 0.3$ & & & \\
\hline & & $1.6 \pm$ & & & 0.3 \\
\hline & & $0.9 \pm 0.3$ & & & \\
\hline & & 0. & & & 0.9 \\
\hline & & $-0.6 \pm 0.6$ & & & \\
\hline GSH & $19.0 \pm 0.2$ & $0.4 \pm 0.4$ & & & 0.3 \\
\hline & & & & & \\
\hline GSH & 1 & $1.4 \pm 0.4$ & & & $.4 \pm 1.1$ \\
\hline & & $-0.3=$ & & & \\
\hline $140+2$ & 1 & $2.0 \pm 0.4$ & & & 0.7 \\
\hline & & & & & \\
\hline GSH & $19.4=$ & $2.2 \pm 1.0$ & & & 1.0 \\
\hline GSH & & \pm 0.3 & & & \\
\hline GSH & & 6 & & & \\
\hline & & & & & \\
\hline GSH & & .7 & & & 0.3 \\
\hline & & & & & 3 \\
\hline & & 4 & & & \\
\hline & & & & & 4 \\
\hline & & 3 & & & \\
\hline & & 4 & & & 3 \\
\hline & & 3 & & & \\
\hline & & 1. & & & 4 \\
\hline & & $-2.5 \pm$ & & & 0.3 \\
\hline GS & & $0.6 \pm 0.4$ & & & 3 \\
\hline & & & & & \\
\hline GS & & $t$ & & & \\
\hline & 2 & & & & \\
\hline GS & & & & & \\
\hline & & & & & \\
\hline & & 1 & & & \\
\hline & & & & & \\
\hline & & & & & \\
\hline & & & & & \\
\hline & & & & & \\
\hline & & & & & \\
\hline & & & & & \\
\hline & & & & & \\
\hline & & -0 & & & \\
\hline & & & & & \\
\hline & & & & & \\
\hline & & & & & \\
\hline & & & & & \\
\hline & & & & & \\
\hline GS & & 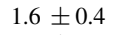 & 26 & & \\
\hline & & & & & \\
\hline & & & & & \\
\hline & & & & & \\
\hline & & & & & \\
\hline & & & & & \\
\hline & & & & & \\
\hline & & & & & \\
\hline & & & & & \\
\hline & & & & & \\
\hline & & & & & \\
\hline & & & & & \\
\hline & & & & & \\
\hline & & & & & \\
\hline & & & & & \\
\hline & & & & $106+$ & \\
\hline
\end{tabular}




\begin{tabular}{|c|c|c|c|c|c|}
\hline Id & Imag & $R-I$ & Id & Imag & $R-I$ \\
\hline GSHCTK J034155+255023 & $19.4 \pm 0.3$ & $-0.4 \pm 0.4$ & GSHCTK J034155+254956 & $19.3 \pm 0.2$ & $0.6 \pm 0.4$ \\
\hline GSHCTK J034156+253912 & $18.7 \pm 0.2$ & $0.8 \pm 0.3$ & GSHCTK J034156+252933 & $19.0 \pm 0.2$ & $0.9 \pm 0.4$ \\
\hline GSHCTK J034156+253934 & $18.4 \pm 0.2$ & $0.2 \pm 0.3$ & GSHCTK J034156+253201 & $19.9 \pm 0.3$ & $-0.1 \pm 0.4$ \\
\hline GSHCTK J034156+253135 & $18.5 \pm 0.2$ & $1.5 \pm 0.4$ & GSHCTK J034156+255405 & $18.6 \pm 0.2$ & $0.9 \pm 0.3$ \\
\hline GSHCTK J034157+253934 & $18.6 \pm 0.2$ & $0.2 \pm 0.3$ & GSHCTK J034158+255328 & $18.2 \pm 0.2$ & $1.3 \pm 0.3$ \\
\hline GSHCTK J034158+254336 & $19.4 \pm 0.2$ & $1.6 \pm 0.6$ & GSHCTK J034158+252026 & $18.9 \pm 0.2$ & $2.0 \pm 0.6$ \\
\hline GSHCTK J034159+254159 & $19.2 \pm 0.2$ & $-0.3 \pm 0.4$ & GSHCTK J034159+253816 & $18.9 \pm 0.2$ & $0.3 \pm 0.3$ \\
\hline GSHCTK J034159+254941 & $18.7 \pm 0.2$ & $0.8 \pm 0.4$ & GSHCTK J034159+254659 & $22.1 \pm 2.0$ & $-1.8 \pm 2.0$ \\
\hline GSHCTK J034159+251918 & $19.3 \pm 0.3$ & $-0.4 \pm 0.4$ & GSHCTK J034159+253606 & $18.7 \pm 0.2$ & $0.8 \pm 0.3$ \\
\hline GSHCTK J034159+253513 & $18.2 \pm 0.2$ & $0.4 \pm 0.3$ & GSHCTK J034159+254722 & $18.7 \pm 0.2$ & $1.6 \pm 0.4$ \\
\hline GSHCTK J034159+254507 & $19.6 \pm 0.3$ & $-0.7 \pm 0.4$ & GSHCTK J034160+253821 & $19.1 \pm 0.2$ & $0.1 \pm 0.3$ \\
\hline GSHCTK J034200+252050 & $17.8 \pm 0.2$ & $0.6 \pm 0.3$ & GSHCTK J034160+254903 & $18.8 \pm 0.2$ & $1.6 \pm 0.4$ \\
\hline GSHCTK J034200+251955 & $17.1 \pm 0.2$ & $1.5 \pm 0.3$ & GSHCTK J034200+255220 & $18.6 \pm 0.2$ & $0.7 \pm 0.3$ \\
\hline GSHCTK J034200+254921 & $18.7 \pm 0.2$ & $1.3 \pm 0.4$ & GSHCTK J034200+253203 & $18.2 \pm 0.2$ & $0.2 \pm 0.3$ \\
\hline GSHCTK J034200+252037 & $19.2 \pm 0.2$ & $0.1 \pm 0.4$ & GSHCTK J034201+253706 & $19.1 \pm 0.2$ & $1.7 \pm 0.5$ \\
\hline GSHCTK J034201+254020 & $18.2 \pm 0.2$ & $-0.0 \pm 0.3$ & GSHCTK J034201+255102 & $18.4 \pm 0.2$ & $0.4 \pm 0.3$ \\
\hline GSHCTK J034202+253814 & $19.6 \pm 0.2$ & $1.5 \pm 0.6$ & GSHCTK J034201+253728 & $20.4 \pm 0.4$ & $1.3 \pm 1.0$ \\
\hline GSHCTK J034202+254445 & $19.8 \pm 0.3$ & $-0.1 \pm 0.4$ & GSHCTK J034202+254624 & $19.2 \pm 0.2$ & $0.6 \pm 0.4$ \\
\hline GSHCTK J034202+253332 & $18.4 \pm 0.2$ & $0.9 \pm 0.3$ & GSHCTK J034202+252028 & $18.8 \pm 0.2$ & $1.3 \pm 0.4$ \\
\hline GSHCTK J034202+255029 & $19.6 \pm 0.3$ & $0.7 \pm 0.5$ & GSHCTK J034202+252929 & $20.3 \pm 0.5$ & $-0.3 \pm 0.6$ \\
\hline GSHCTK J034202+254618 & $19.7 \pm 0.3$ & $0.7 \pm 0.5$ & GSHCTK J034202+253852 & $19.8 \pm 0.3$ & $-0.3 \pm 0.4$ \\
\hline GSHCTK J034203+253903 & $18.9 \pm 0.2$ & $0.2 \pm 0.3$ & GSHCTK J034203+253002 & $18.9 \pm 0.2$ & $1.7 \pm 0.4$ \\
\hline GSHCTK J034203+252210 & $18.4 \pm 0.2$ & $2.0 \pm 0.4$ & GSHCTK J034203+255118 & $17.7 \pm 0.2$ & $0.9 \pm 0.3$ \\
\hline GSHCTK J034203+254345 & $19.1 \pm 0.2$ & $1.0 \pm 0.4$ & GSHCTK J034203+254622 & $21.9 \pm 1.7$ & $-1.3 \pm 1.8$ \\
\hline GSHCTK J034203+255140 & $18.8 \pm 0.2$ & $1.1 \pm 0.4$ & GSHCTK J034203+254425 & $18.5 \pm 0.2$ & $1.1 \pm 0.3$ \\
\hline GSHCTK J034204+255104 & $19.1 \pm 0.2$ & $1.1 \pm 0.4$ & GSHCTK J034204+253819 & $19.1 \pm 0.2$ & $0.9 \pm 0.4$ \\
\hline GSHCTK J034204+252856 & $19.3 \pm 0.3$ & $1.3 \pm 0.5$ & GSHCTK J034204+253746 & $23.3 \pm 5.6$ & $-2.3 \pm 5.6$ \\
\hline GSHCTK J034204+254956 & $17.8 \pm 0.2$ & $0.4 \pm 0.3$ & GSHCTK J034204+252958 & $18.5 \pm 0.2$ & $1.8 \pm 0.4$ \\
\hline GSHCTK J034204+252630 & $18.5 \pm 0.2$ & $1.7 \pm 0.4$ & GSHCTK J034204+252340 & $19.4 \pm 0.3$ & $0.4 \pm 0.4$ \\
\hline GSHCTK J034204+253449 & $18.6 \pm 0.2$ & $0.9 \pm 0.3$ & GSHCTK J034204+252652 & $18.6 \pm 0.2$ & $1.6 \pm 0.4$ \\
\hline GSHCTK J034205+254531 & $17.3 \pm 0.2$ & $1.1 \pm 0.3$ & GSHCTK J034205+252407 & $18.4 \pm 0.2$ & $1.9 \pm 0.4$ \\
\hline GSHCTK J034205+251913 & $18.9 \pm 0.2$ & $0.1 \pm 0.4$ & GSHCTK J034205+255120 & $18.1 \pm 0.2$ & $1.1 \pm 0.3$ \\
\hline GSHCTK J034205+252435 & $19.1 \pm 0.2$ & $1.1 \pm 0.4$ & GSHCTK J034205+252221 & $17.5 \pm 0.2$ & $1.4 \pm 0.3$ \\
\hline GSHCTK J034205+251931 & $18.5 \pm 0.2$ & $0.2 \pm 0.3$ & GSHCTK J034205+255020 & $17.6 \pm 0.2$ & $0.9 \pm 0.3$ \\
\hline GSHCTK J034205+253712 & $19.9 \pm 0.3$ & $-0.3 \pm 0.4$ & GSHCTK J034205+253750 & $22.1 \pm 2.1$ & $-1.5 \pm 2.1$ \\
\hline GSHCTK J034205+253208 & $20.7 \pm 0.6$ & $0.9 \pm 1.0$ & GSHCTK J034205+252227 & $17.6 \pm 0.2$ & $=0.3$ \\
\hline GSHCTK J034205+251922 & $18.7 \pm 0.2$ & $0.0 \pm 0.3$ & GSHCTK J034206+255155 & $20.4 \pm 0.6$ & $0.9 \pm 0.9$ \\
\hline GSHCTK J034206+254432 & $20.1 \pm 0.4$ & $-0.4 \pm 0.5$ & GSHCTK J034206+252639 & $17.8 \pm 0.2$ & $5.3 \pm 3.9$ \\
\hline GSHCTK J034206+252520 & $18.5 \pm 0.2$ & $1.2 \pm 0.4$ & GSHCTK J034206+253338 & $20.6 \pm 0.5$ & $-0.9 \pm 0.6$ \\
\hline GSHCTK J034206+252203 & $17.4 \pm 0.2$ & $0.7 \pm 0.3$ & GSHCTK J034206+252442 & $18.3 \pm 0.2$ & $1.2 \pm 0.3$ \\
\hline GSHCTK J034206+252433 & $18.6 \pm 0.2$ & $1.1 \pm 0.4$ & GSHCTK J034207+254739 & $20.9 \pm 0.7$ & $-0.3 \pm 0.9$ \\
\hline GSHCTK J034207+252555 & $18.5 \pm 0.2$ & $0.7 \pm 0.3$ & GSHCTK J034207+255142 & $19.5 \pm 0.3$ & $0.3 \pm 0.4$ \\
\hline GSHCTK J034207+252810 & $19.7 \pm 0.3$ & $0.1 \pm 0.4$ & GSHCTK J034207+252548 & $18.6 \pm 0.2$ & $0.5 \pm 0.3$ \\
\hline GSHCTK J034208+252411 & $19.0 \pm 0.2$ & $1.6 \pm 0.5$ & GSHCTK J034208+253821 & $18.1 \pm 0.2$ & $1.1 \pm 0.3$ \\
\hline GSHCTK J034208+252555 & $17.9 \pm 0.2$ & $0.5 \pm 0.3$ & GSHCTK J034209+253540 & $19.2 \pm 0.2$ & $1.0 \pm 0.4$ \\
\hline GSHCTK J034209+252734 & $17.6 \pm 0.2$ & $0.8 \pm 0.3$ & GSHCTK J034209+252239 & $19.4 \pm 0.3$ & $0.8 \pm 0.4$ \\
\hline GSHCTK J034209+254640 & $18.5 \pm 0.2$ & $3.4 \pm 1.1$ & GSHCTK J034209+251933 & $19.0 \pm 0.2$ & $-0.4 \pm 0.3$ \\
\hline GSHCTK J034209+252900 & $19.0 \pm 0.2$ & $0.9 \pm 0.4$ & GSHCTK J034210+252732 & $18.7 \pm 0.2$ & $0.1 \pm 0.3$ \\
\hline GSHCTK J034209+252637 & $19.1 \pm 0.2$ & $-0.3 \pm 0.3$ & GSHCTK J034210+252356 & $18.3 \pm 0.2$ & $1.7 \pm 0.4$ \\
\hline GSHCTK J034210+254208 & $17.5 \pm 0.2$ & $1.0 \pm 0.3$ & GSHCTK J034210+253414 & $19.2 \pm 0.2$ & $0.2 \pm 0.4$ \\
\hline GSHCTK J034210+254923 & $19.0 \pm 0.2$ & $1.1 \pm 0.4$ & GSHCTK J034210+254337 & $18.9 \pm 0.2$ & $0.6 \pm 0.3$ \\
\hline GSHCTK J034210+254159 & $17.9 \pm 0.2$ & $0.7 \pm 0.3$ & GSHCTK J034210+254806 & $18.4 \pm 0.2$ & $1.5 \pm 0.4$ \\
\hline GSHCTK J034210+253615 & $19.1 \pm 0.2$ & $-0.0 \pm 0.4$ & GSHCTK J034211+254531 & $19.3 \pm 0.2$ & $0.6 \pm 0.4$ \\
\hline GSHCTK J034210+253845 & $19.1 \pm 0.2$ & $1.3 \pm 0.4$ & GSHCTK J034210+252847 & $19.3 \pm 0.2$ & $0.9 \pm 0.4$ \\
\hline GSHCTK J034211+254416 & $18.5 \pm 0.2$ & $1.1 \pm 0.3$ & GSHCTK J034211+254131 & $19.6 \pm 0.3$ & $0.9 \pm 0.4$ \\
\hline GSHCTK J034211+254925 & $19.0 \pm 0.2$ & $0.7 \pm 0.4$ & GSHCTK J034211+254401 & $18.5 \pm 0.2$ & $0.3 \pm 0.3$ \\
\hline GSHCTK J034211+255027 & $18.7 \pm 0.2$ & $0.9 \pm 0.3$ & GSHCTK J034211+253354 & $19.5 \pm 0.2$ & $1.5 \pm 0.6$ \\
\hline GSHCTK J034211+251924 & $18.0 \pm 0.2$ & $0.8 \pm 0.3$ & GSHCTK J034212+255248 & $18.7 \pm 0.2$ & $1.7 \pm 0.4$ \\
\hline GSHCTK J034212+253159 & $18.4 \pm 0.2$ & $0.8 \pm 0.3$ & GSHCTK J034212+252407 & $19.2 \pm 0.2$ & $1.4 \pm 0.5$ \\
\hline GSHCTK J034212+252258 & $19.3 \pm 0.2$ & $0.7 \pm 0.4$ & GSHCTK J034212+253252 & $19.2 \pm 0.2$ & $0.5 \pm 0.4$ \\
\hline GSHCTK J034212+255226 & $19.4 \pm 0.3$ & $1.2 \pm 0.5$ & GSHCTK J034212+252551 & $19.2 \pm 0.2$ & $1.4 \pm 0.5$ \\
\hline GSHCTK J034212+252610 & $19.0 \pm 0.2$ & $1.7 \pm 0.5$ & GSHCTK J034212+252028 & $18.4 \pm 0.2$ & $0.6 \pm 0.3$ \\
\hline GSHCTK J034212+252522 & $18.4 \pm 0.2$ & $1.5 \pm 0.4$ & GSHCTK J034213+253301 & $20.3 \pm 0.4$ & $-1.0 \pm 0.5$ \\
\hline GSHCTK J034213+252900 & $18.5 \pm 0.2$ & $1.4 \pm 0.4$ & GSHCTK J034213+252509 & $18.9 \pm 0.2$ & $2.2 \pm 0.6$ \\
\hline GSHCTK J034213+252418 & $20.4 \pm 0.5$ & $0.1 \pm 0.6$ & GSHCTK J034213+252325 & $18.0 \pm 0.2$ & $3.1 \pm 0.6$ \\
\hline GSHCTK J034213+255012 & $19.1 \pm 0.2$ & $0.6 \pm 0.4$ & GSHCTK J034213+254905 & $19.0 \pm 0.2$ & $-0.4 \pm 0.3$ \\
\hline GSHCTK J034214+254414 & $18.9 \pm 0.2$ & $1.9 \pm 0.5$ & GSHCTK J034214+252927 & $21.2 \pm 1.0$ & \\
\hline GSHCTK J034214+252320 & $18.0 \pm 0.2$ & $1.4 \pm 0.3$ & GSHCTK J034214+253936 & $19.0 \pm 0.2$ & $0.6 \pm 0.3$ \\
\hline GSHCTK J034215+255202 & $19.3 \pm 0.3$ & $1.2 \pm 0.5$ & GSHCTK J034215+253343 & $18.6 \pm 0.2$ & $0.9 \pm 0.3$ \\
\hline GSHCTK J034215+252544 & $19.0 \pm 0.2$ & $0.7 \pm 0.4$ & GSHCTK J034215+254556 & $18.4 \pm 0.2$ & $0.4 \pm 0.3$ \\
\hline GSHCTK J034215+252714 & $17.6 \pm 0.2$ & $2.9 \pm 0.4$ & GSHCTK J034215+253022 & $17.8 \pm 0.2$ & $1.3 \pm 0.3$ \\
\hline GSHCTK J034215+252458 & $18.4 \pm 0.2$ & $0.4 \pm 0.3$ & GSHCTK J034215+252814 & $19.1 \pm 0.2$ & $0.1 \pm 0.4$ \\
\hline GSHCTK J034215+252537 & $19.8 \pm 0.3$ & $0.4 \pm 0.5$ & GSHCTK J034216+254040 & $19.8 \pm 0.3$ & $0.6 \pm 0.5$ \\
\hline GSHCTK J034216+253648 & $18.7 \pm 0.2$ & $1.0 \pm 0.4$ & GSHCTK J034216+253726 & $19.0 \pm 0.2$ & $0.8 \pm 0.4$ \\
\hline GSHCTK J034216+252555 & $18.4 \pm 0.2$ & $0.7 \pm 0.3$ & GSHCTK J034216+252852 & $17.6 \pm 0.2$ & $0.4 \pm 0.3$ \\
\hline GSHCTK J034217+253732 & $19.7 \pm 0.3$ & $0.7 \pm 0.5$ & GSHCTK J034217+254018 & $19.0 \pm 0.2$ & $0.9 \pm 0.4$ \\
\hline GSHCTK J034217+255326 & $17.7 \pm 0.2$ & $1.7 \pm 0.3$ & GSHCTK J034217+255001 & $18.9 \pm 0.2$ & $0.3 \pm 0.3$ \\
\hline GSHCTK J034217+253206 & $18.2 \pm 0.2$ & $0.5 \pm 0.3$ & GSHCTK J034217+252132 & $18.4 \pm 0.2$ & \\
\hline GSHCTK J034217+254129 & $18.4 \pm 0.2$ & $1.8 \pm 0.4$ & GSHCTK J034218+253830 & $19.5 \pm 0.3$ & $0.8 \pm 0.4$ \\
\hline GSHCTK J034218+254458 & $18.8 \pm 0.2$ & $0.9 \pm 0.4$ & GSHCTK J034218+253940 & $19.5 \pm 0.3$ & \\
\hline GSHCTK J034218+254852 & $19.5 \pm 0.3$ & $1.7 \pm 0.7$ & GSHCTK J034218+253037 & $19.5 \pm 0.3$ & $1.6 \pm$ \\
\hline GSHCTK J034218+254609 & $17.3 \pm 0.2$ & $0.8 \pm 0.3$ & GSHCTK J034219+254109 & $19.0 \pm 0.2$ & \\
\hline GSHCTK J034219+252301 & $19.4 \pm 0.3$ & $0.2 \pm 0.4$ & GSHCTK J034219+251907 & $19.1 \pm 0.3$ & $0.7 \pm 0.4$ \\
\hline
\end{tabular}




\begin{tabular}{|c|c|c|c|c|c|}
\hline Id & Imag & $R-I$ & Id & Imag & $R-I$ \\
\hline GSHCTK J034219+254418 & $18.0 \pm 0.2$ & $0.7 \pm 0.3$ & GSHCTK J034219+253759 & $19.5 \pm 0.3$ & $0.2 \pm 0.4$ \\
\hline GSHCTK J034220+254133 & $19.3 \pm 0.2$ & $0.2 \pm 0.4$ & GSHCTK J034220+255107 & $18.2 \pm 0.2$ & $0.8 \pm 0.3$ \\
\hline GSHCTK J034220+254321 & $17.6 \pm 0.2$ & $0.8 \pm 0.3$ & GSHCTK J034220+252424 & $21.4 \pm 1.3$ & $0.1 \pm 1.6$ \\
\hline GSHCTK J034220+255140 & $18.6 \pm 0.2$ & $2.0 \pm 0.5$ & GSHCTK J034220+252307 & $18.2 \pm 0.2$ & $0.9 \pm 0.3$ \\
\hline GSHCTK J034220+255226 & $18.4 \pm 0.2$ & $1.7 \pm 0.4$ & GSHCTK J034220+254343 & $18.8 \pm 0.2$ & $0.9 \pm 0.4$ \\
\hline GSHCTK J034220+254533 & $17.5 \pm 0.2$ & $1.0 \pm 0.3$ & GSHCTK J034221+253956 & $20.0 \pm 0.3$ & $0.3 \pm 0.5$ \\
\hline GSHCTK J034221+252827 & $18.7 \pm 0.2$ & $1.1 \pm 0.4$ & GSHCTK J034221+253152 & $18.6 \pm 0.2$ & $0.9 \pm 0.3$ \\
\hline GSHCTK J034221+252035 & $17.5 \pm 0.2$ & $0.7 \pm 0.3$ & GSHCTK J034221+252444 & $19.9 \pm 0.3$ & $0.8 \pm 0.6$ \\
\hline GSHCTK J034221+254826 & $20.8 \pm 0.7$ & $-1.1 \pm 0.8$ & GSHCTK J034222+254124 & $20.0 \pm 0.3$ & $1.0 \pm 0.6$ \\
\hline GSHCTK J034222+252323 & $18.0 \pm 0.2$ & $1.5 \pm 0.3$ & GSHCTK J034222+253259 & $20.1 \pm 0.4$ & $-0.5 \pm 0.5$ \\
\hline GSHCTK J034222+253726 & $17.2 \pm 0.2$ & $1.0 \pm 0.3$ & GSHCTK J034222+254947 & $19.0 \pm 0.2$ & $1.3 \pm 0.4$ \\
\hline GSHCTK J034222+253031 & $21.3 \pm 1.2$ & $-1.5 \pm 1.3$ & GSHCTK J034222+253217 & $18.6 \pm 0.2$ & $0.5 \pm 0.3$ \\
\hline GSHCTK J034222+254027 & $19.3 \pm 0.2$ & $1.0 \pm 0.4$ & GSHCTK J034222+254908 & $19.1 \pm 0.2$ & $0.9 \pm 0.4$ \\
\hline GSHCTK J034223+254631 & $17.0 \pm 0.2$ & $0.8 \pm 0.3$ & GSHCTK J034223+252810 & $21.1 \pm 0.8$ & $-2.0 \pm 0.8$ \\
\hline GSHCTK J034223+253916 & $19.8 \pm 0.3$ & $0.5 \pm 0.5$ & GSHCTK J034223+254000 & $18.5 \pm 0.2$ & $0.5 \pm 0.3$ \\
\hline GSHCTK J034224+253737 & $19.3 \pm 0.2$ & $0.2 \pm 0.4$ & GSHCTK J034224+253816 & $21.0 \pm 0.7$ & $-0.1 \pm 0.8$ \\
\hline GSHCTK J034224+252918 & $20.7 \pm 0.6$ & $-1.4 \pm 0.7$ & GSHCTK J034224+254739 & $18.3 \pm 0.2$ & $0.6 \pm 0.3$ \\
\hline GSHCTK J034224+253546 & $18.5 \pm 0.2$ & $0.8 \pm 0.3$ & GSHCTK J034224+252701 & $19.1 \pm 0.2$ & $1.2 \pm 0.4$ \\
\hline GSHCTK J034224+253434 & $18.7 \pm 0.2$ & $1.4 \pm 0.4$ & GSHCTK J034224+254128 & $20.4 \pm 0.4$ & $-0.3 \pm 0.5$ \\
\hline GSHCTK J034224+255005 & $19.8 \pm 0.3$ & $0.7 \pm 0.5$ & GSHCTK J034225+252019 & $20.1 \pm 0.4$ & $-0.8 \pm 0.5$ \\
\hline GSHCTK J034225+255250 & $17.9 \pm 0.2$ & $0.8 \pm 0.3$ & GSHCTK J034225+252509 & $19.7 \pm 0.3$ & $1.1 \pm 0.6$ \\
\hline GSHCTK J034225+254458 & $18.3 \pm 0.2$ & $0.5 \pm 0.3$ & GSHCTK J034225+252044 & $19.3 \pm 0.2$ & $-0.7 \pm 0.4$ \\
\hline GSHCTK J034225+252314 & $18.9 \pm 0.2$ & $0.2 \pm 0.3$ & GSHCTK J034225+252725 & $18.7 \pm 0.2$ & $0.7 \pm 0.3$ \\
\hline GSHCTK J034225+254434 & $19.7 \pm 0.3$ & $1.0 \pm 0.5$ & GSHCTK J034226+252152 & $18.6 \pm 0.2$ & $1.3 \pm 0.4$ \\
\hline GSHCTK J034226+255217 & $18.6 \pm 0.2$ & $1.6 \pm 0.4$ & GSHCTK J034226+255259 & $19.6 \pm 0.3$ & $1.4 \pm 0.5$ \\
\hline GSHCTK J034226+255239 & $17.7 \pm 0.2$ & $1.1 \pm 0.3$ & GSHCTK J034226+251929 & $18.5 \pm 0.2$ & $1.5 \pm 0.4$ \\
\hline GSHCTK J034226+252849 & $18.0 \pm 0.2$ & $1.1 \pm 0.3$ & GSHCTK J034227+253719 & $19.3 \pm 0.2$ & $1.8 \pm 0.6$ \\
\hline GSHCTK J034227+254219 & $19.1 \pm 0.2$ & $1.1 \pm 0.4$ & GSHCTK J034227+254737 & $19.4 \pm 0.3$ & $1.3 \pm 0.5$ \\
\hline GSHCTK J034227+252307 & $17.4 \pm 0.2$ & $1.5 \pm 0.3$ & GSHCTK J034227+252931 & $19.4 \pm 0.3$ & $1.7 \pm 0.6$ \\
\hline GSHCTK J034227+253533 & $18.4 \pm 0.2$ & $1.5 \pm 0.4$ & GSHCTK J034227+254558 & $19.7 \pm 0.3$ & $1.0 \pm 0.5$ \\
\hline GSHCTK J034227+253113 & $19.6 \pm 0.3$ & $1.9 \pm 0.7$ & GSHCTK J034227+253411 & $21.5 \pm 1.5$ & $-1.7 \pm 1.5$ \\
\hline GSHCTK J034227+254013 & $19.3 \pm 0.2$ & $1.2 \pm 0.4$ & GSHCTK J034228+252511 & $17.0 \pm 0.2$ & $1.6 \pm 0.3$ \\
\hline GSHCTK J034228+252602 & $19.3 \pm 0.3$ & $0.0 \pm 0.4$ & GSHCTK J034228+252949 & $18.5 \pm 0.2$ & $1.9 \pm 0.4$ \\
\hline GSHCTK J034228+253524 & $18.4 \pm 0.2$ & $1.1 \pm 0.3$ & GSHCTK J034228+255155 & $17.7 \pm 0.2$ & $0.7 \pm 0.3$ \\
\hline GSHCTK J034228+253509 & $18.3 \pm 0.2$ & $2.1 \pm 0.4$ & GSHCTK J034229+253226 & $19.0 \pm 0.2$ & $0.7 \pm 0.4$ \\
\hline GSHCTK J034229+253825 & $19.7 \pm 0.3$ & $0.2 \pm 0.4$ & GSHCTK J034229+254022 & $19.8 \pm 0.3$ & $-0.2 \pm 0.4$ \\
\hline GSHCTK J034229+252725 & $19.4 \pm 0.3$ & $1.5 \pm 0.5$ & GSHCTK J034229+254724 & $19.1 \pm 0.2$ & $1.2 \pm 0.4$ \\
\hline GSHCTK J034229+254458 & $19.7 \pm 0.3$ & $-0.4 \pm 0.4$ & GSHCTK J034229+254812 & $17.9 \pm 0.2$ & $0.5 \pm 0.3$ \\
\hline GSHCTK J034229+252608 & $18.9 \pm 0.2$ & $-0.1 \pm 0.3$ & GSHCTK J034229+252825 & $20.6 \pm 0.6$ & $2.0 \pm 2.1$ \\
\hline GSHCTK J034229+254350 & $18.9 \pm 0.2$ & $0.6 \pm 0.3$ & GSHCTK J034229+252048 & $19.5 \pm 0.3$ & $0.4 \pm 0.4$ \\
\hline GSHCTK J034229+252117 & $18.1 \pm 0.2$ & $0.6 \pm 0.3$ & GSHCTK J034229+252130 & $18.7 \pm 0.2$ & $1.8 \pm 0.5$ \\
\hline GSHCTK J034229+252619 & $18.5 \pm 0.2$ & $0.3 \pm 0.3$ & GSHCTK J034230+254235 & $17.6 \pm 0.2$ & $1.3 \pm 0.3$ \\
\hline GSHCTK J034230+255317 & $18.3 \pm 0.2$ & $1.1 \pm 0.3$ & GSHCTK J034230+254925 & $19.2 \pm 0.2$ & $0.1 \pm 0.4$ \\
\hline GSHCTK J034230+253219 & $20.1 \pm 0.4$ & $-0.1 \pm 0.5$ & GSHCTK J034230+254336 & $19.6 \pm 0.2$ & $0.2 \pm 0.4$ \\
\hline GSHCTK J034230+253847 & $18.8 \pm 0.2$ & $0.4 \pm 0.3$ & GSHCTK J034230+252825 & $19.1 \pm 0.2$ & $1.0 \pm 0.4$ \\
\hline GSHCTK J034230+252225 & $18.2 \pm 0.2$ & $1.7 \pm 0.4$ & GSHCTK J034230+253958 & $19.5 \pm 0.2$ & $1.2 \pm 0.5$ \\
\hline GSHCTK J034231+253914 & $18.8 \pm 0.2$ & $1.3 \pm 0.4$ & GSHCTK J034231+255160 & $18.7 \pm 0.2$ & $0.2 \pm 0.3$ \\
\hline GSHCTK J034231+253004 & $18.1 \pm 0.2$ & $0.5 \pm 0.3$ & GSHCTK J034231+255224 & $18.3 \pm 0.2$ & $1.5 \pm 0.3$ \\
\hline GSHCTK J034231+254815 & $19.4 \pm 0.3$ & $-0.1 \pm 0.4$ & GSHCTK J034231+252621 & $18.1 \pm 0.2$ & $0.6 \pm 0.3$ \\
\hline GSHCTK J034232+252329 & $18.8 \pm 0.2$ & $1.4 \pm 0.4$ & GSHCTK J034232+253858 & $20.1 \pm 0.3$ & $0.2 \pm 0.5$ \\
\hline GSHCTK J034232+254803 & $19.3 \pm 0.2$ & $0.3 \pm 0.4$ & GSHCTK J034233+252340 & $18.5 \pm 0.2$ & $1.7 \pm 0.4$ \\
\hline GSHCTK J034233+252022 & $18.4 \pm 0.2$ & $1.7 \pm 0.4$ & GSHCTK J034233+255146 & $18.3 \pm 0.2$ & $1.1 \pm 0.3$ \\
\hline GSHCTK J034233+252002 & $17.2 \pm 0.2$ & $0.8 \pm 0.3$ & GSHCTK J034233+254312 & $20.3 \pm 0.4$ & $-0.5 \pm 0.5$ \\
\hline GSHCTK J034233+254414 & $19.9 \pm 0.3$ & $2.2 \pm 1.3$ & GSHCTK J034233+252522 & $17.8 \pm 0.2$ & $0.8 \pm 0.3$ \\
\hline GSHCTK J034234+252617 & $19.2 \pm 0.2$ & $0.6 \pm 0.4$ & GSHCTK J034234+253704 & $19.0 \pm 0.2$ & $0.7 \pm 0.4$ \\
\hline GSHCTK J034234+252316 & $18.6 \pm 0.2$ & $1.4 \pm 0.4$ & GSHCTK J034234+254629 & $21.9 \pm 1.8$ & $-1.1 \pm 1.9$ \\
\hline GSHCTK J034234+255317 & $18.1 \pm 0.2$ & $3.2 \pm 0.6$ & GSHCTK J034235+254456 & $20.0 \pm 0.3$ & $0.1 \pm 0.5$ \\
\hline GSHCTK J034235+253334 & $18.9 \pm 0.2$ & $-0.0 \pm 0.3$ & GSHCTK J034235+253728 & $20.0 \pm 0.3$ & $-0.0 \pm 0.4$ \\
\hline GSHCTK J034235+253020 & $22.1 \pm 2.7$ & $-1.5 \pm 2.7$ & GSHCTK J034235+254106 & $20.0 \pm 0.3$ & $-1.3 \pm 0.4$ \\
\hline GSHCTK J034236+252247 & $19.7 \pm 0.3$ & $0.2 \pm 0.5$ & GSHCTK J034236+253243 & $19.8 \pm 0.3$ & $0.2 \pm 0.4$ \\
\hline GSHCTK J034236+253259 & $19.9 \pm 0.3$ & $-0.6 \pm 0.4$ & GSHCTK J034236+254759 & $20.3 \pm 0.4$ & $-0.3 \pm 0.5$ \\
\hline GSHCTK J034236+252157 & $19.9 \pm 0.4$ & $0.7 \pm 0.6$ & GSHCTK J034236+253641 & $20.7 \pm 0.6$ & $-0.8 \pm 0.7$ \\
\hline GSHCTK J034237+253841 & $19.7 \pm 0.3$ & $0.6 \pm 0.5$ & GSHCTK J034236+254401 & $19.2 \pm 0.2$ & $1.2 \pm 0.5$ \\
\hline GSHCTK J034236+254633 & $18.3 \pm 0.2$ & $0.7 \pm 0.3$ & GSHCTK J034237+255034 & $18.8 \pm 0.2$ & $1.6 \pm 0.4$ \\
\hline GSHCTK J034237+253033 & $20.0 \pm 0.4$ & $0.4 \pm 0.5$ & GSHCTK J034237+252756 & $18.7 \pm 0.2$ & $0.7 \pm 0.3$ \\
\hline GSHCTK J034237+254115 & $17.3 \pm 0.2$ & $0.5 \pm 0.3$ & GSHCTK J034237+255244 & $18.5 \pm 0.2$ & $1.3 \pm 0.4$ \\
\hline GSHCTK J034237+252314 & $18.6 \pm 0.2$ & & GSHCTK J034237+254531 & & $0.7 \pm 0.4$ \\
\hline GSHCTK J034237+254609 & $17.2 \pm 0.2$ & $0.7 \pm 0.3$ & GSHCTK J034237+255100 & $18.8 \pm 0.2$ & $1.1 \pm 0.4$ \\
\hline GSHCTK J034237+253400 & $16.7 \pm 0.2$ & $1.0 \pm 0.3$ & GSHCTK J034238+253825 & $21.5 \pm 1.1$ & $-1.4 \pm 1.1$ \\
\hline GSHCTK J034238+254560 & $19.4 \pm 0.3$ & $0.6 \pm 0.4$ & GSHCTK J034238+254713 & $18.1 \pm 0.2$ & $0.4 \pm 0.3$ \\
\hline GSHCTK J034238+253938 & $18.7 \pm 0.2$ & $1.5 \pm 0.4$ & GSHCTK J034238+253316 & $18.6 \pm 0.2$ & $1.3 \pm 0.4$ \\
\hline GSHCTK J034238+255138 & $18.9 \pm 0.2$ & $0.8 \pm 0.4$ & GSHCTK J034239+252154 & $18.2 \pm 0.2$ & $1.8 \pm 0.4$ \\
\hline GSHCTK J034238+253031 & $18.2 \pm 0.2$ & $1.7 \pm 0.4$ & GSHCTK J034238+253748 & $17.9 \pm 0.2$ & \\
\hline GSHCTK J034239+253526 & $18.3 \pm 0.2$ & $0.1 \pm 0.3$ & GSHCTK J034239+254046 & $19.5 \pm 0.3$ & $-0.3 \pm 0.4$ \\
\hline GSHCTK J034239+254930 & $20.6 \pm 0.6$ & $-0.7 \pm 0.7$ & GSHCTK J034239+254235 & $19.8 \pm 0.3$ & $1.6 \pm 0.8$ \\
\hline GSHCTK J034239+255412 & $20.9 \pm 0.6$ & $-1.1 \pm 0.7$ & GSHCTK J034239+253009 & $18.7 \pm 0.2$ & $1.2 \pm 0.4$ \\
\hline GSHCTK J034239+253604 & $18.9 \pm 0.2$ & $1.2 \pm 0.4$ & GSHCTK J034239+251918 & $18.5 \pm 0.2$ & $0.6 \pm 0.3$ \\
\hline GSHCTK J034239+253717 & $17.6 \pm 0.2$ & $1.4 \pm 0.3$ & GSHCTK J034240+252533 & $19.4 \pm 0.3$ & $1.4 \pm$ \\
\hline GSHCTK J034240+254142 & $17.9 \pm 0.2$ & $1.3 \pm 0.3$ & GSHCTK J034240+254735 & $18.3 \pm 0.2$ & $0.6 \pm 0.3$ \\
\hline GSHCTK J034240+252624 & $17.3 \pm 0.2$ & & GSHCTK J034240+254325 & & \\
\hline GSHCTK J034240+253544 & $20.0 \pm 0.4$ & $-0.3 \pm 0.5$ & GSHCTK J034241+254555 & $17.2 \pm 0.2$ & $0.5 \pm$ \\
\hline GSHCTK J034241+254646 & $18.3 \pm 0.2$ & & GSHCTK J034242+252535 & 19. & \\
\hline GSHCTK J034241+255246 & $19.5 \pm 0.3$ & $1.8 \pm 0.7$ & GSHCTK J034242+252820 & $17.5 \pm 0.2$ & $0.8 \pm 0.3$ \\
\hline
\end{tabular}




\begin{tabular}{|c|c|c|c|c|c|}
\hline Id & Imag & $R-I$ & Id & Imag & $R-I$ \\
\hline GSHCTK J034242+254137 & $18.7 \pm 0.2$ & $1.0 \pm 0.4$ & GSHCTK J034242+254710 & $18.2 \pm 0.2$ & $0.9 \pm 0.3$ \\
\hline GSHCTK J034242+254744 & $18.3 \pm 0.2$ & $1.0 \pm 0.3$ & GSHCTK J034242+254131 & $17.3 \pm 0.2$ & $1.1 \pm 0.3$ \\
\hline GSHCTK J034242+255408 & $18.9 \pm 0.2$ & $0.2 \pm 0.3$ & GSHCTK J034242+255352 & $18.3 \pm 0.2$ & $0.8 \pm 0.3$ \\
\hline GSHCTK J034242+253221 & $18.8 \pm 0.2$ & $1.7 \pm 0.4$ & GSHCTK J034243+253247 & $19.6 \pm 0.3$ & $-0.2 \pm 0.4$ \\
\hline GSHCTK J034243+251933 & $18.3 \pm 0.2$ & $0.7 \pm 0.3$ & GSHCTK J034243+252115 & $18.4 \pm 0.2$ & $1.4 \pm 0.4$ \\
\hline GSHCTK J034243+252506 & $18.6 \pm 0.2$ & $1.1 \pm 0.4$ & GSHCTK J034243+252557 & $19.1 \pm 0.2$ & $0.6 \pm 0.4$ \\
\hline GSHCTK J034244+252435 & $18.8 \pm 0.2$ & $0.1 \pm 0.3$ & GSHCTK J034244+251902 & $18.6 \pm 0.2$ & $0.3 \pm 0.3$ \\
\hline GSHCTK J034244+255257 & $20.3 \pm 0.6$ & $1.3 \pm 1.0$ & GSHCTK J034244+251909 & $18.1 \pm 0.2$ & $0.3 \pm 0.3$ \\
\hline GSHCTK J034244+253741 & $18.2 \pm 0.2$ & $0.3 \pm 0.3$ & GSHCTK J034244+254852 & $18.9 \pm 0.2$ & $1.3 \pm 0.4$ \\
\hline GSHCTK J034245+252216 & $19.1 \pm 0.2$ & $0.5 \pm 0.4$ & GSHCTK J034245+252827 & $23.2 \pm 5.9$ & $-2.6 \pm 5.9$ \\
\hline GSHCTK J034245+254201 & $19.0 \pm 0.2$ & $0.0 \pm 0.3$ & GSHCTK J034245+254449 & $19.3 \pm 0.3$ & $1.2 \pm 0.5$ \\
\hline GSHCTK J034245+252758 & $18.3 \pm 0.2$ & $0.7 \pm 0.3$ & GSHCTK J034245+253520 & $18.9 \pm 0.2$ & $0.5 \pm 0.3$ \\
\hline GSHCTK J034245+253756 & $19.9 \pm 0.3$ & $0.6 \pm 0.5$ & GSHCTK J034246+253520 & $18.8 \pm 0.2$ & $0.6 \pm 0.3$ \\
\hline GSHCTK J034246+254916 & $18.7 \pm 0.2$ & $1.3 \pm 0.4$ & GSHCTK J034246+253958 & $17.6 \pm 0.2$ & $1.5 \pm 0.3$ \\
\hline GSHCTK J034246+255036 & $17.6 \pm 0.2$ & $0.5 \pm 0.3$ & GSHCTK J034246+253137 & $19.0 \pm 0.2$ & $1.8 \pm 0.5$ \\
\hline GSHCTK J034246+252015 & $18.4 \pm 0.2$ & $1.4 \pm 0.4$ & GSHCTK J034247+255009 & $19.4 \pm 0.3$ & $1.6 \pm 0.6$ \\
\hline GSHCTK J034247+252309 & $19.2 \pm 0.2$ & $1.5 \pm 0.5$ & GSHCTK J034247+253513 & $19.2 \pm 0.2$ & $0.9 \pm 0.4$ \\
\hline GSHCTK J034247+254659 & $19.2 \pm 0.2$ & $0.4 \pm 0.4$ & GSHCTK J034247+253042 & $18.6 \pm 0.2$ & $0.7 \pm 0.3$ \\
\hline GSHCTK J034247+253258 & $17.7 \pm 0.2$ & $0.5 \pm 0.3$ & GSHCTK J034247+252232 & $18.2 \pm 0.2$ & $2.0 \pm 0.4$ \\
\hline GSHCTK J034248+254352 & $20.7 \pm 0.7$ & $0.6 \pm 0.9$ & GSHCTK J034248+255337 & $18.3 \pm 0.2$ & $1.8 \pm 0.4$ \\
\hline GSHCTK J034248+253455 & $19.2 \pm 0.2$ & $0.3 \pm 0.4$ & GSHCTK J034248+254029 & $22.6 \pm 3.5$ & $-3.2 \pm 3.5$ \\
\hline GSHCTK J034248+254657 & $19.0 \pm 0.2$ & $1.6 \pm 0.5$ & GSHCTK J034248+254332 & $17.3 \pm 0.2$ & $0.4 \pm 0.3$ \\
\hline GSHCTK J034248+254622 & $18.3 \pm 0.2$ & $1.7 \pm 0.4$ & GSHCTK J034248+253429 & $20.4 \pm 0.6$ & $-0.0 \pm 0.7$ \\
\hline GSHCTK J034248+254128 & $17.8 \pm 0.2$ & $0.7 \pm 0.3$ & GSHCTK J034248+254633 & $18.5 \pm 0.2$ & $1.2 \pm 0.4$ \\
\hline GSHCTK J034249+252723 & $19.3 \pm 0.2$ & $1.0 \pm 0.4$ & GSHCTK J034249+254912 & $19.3 \pm 0.3$ & $1.4 \pm 0.5$ \\
\hline GSHCTK J034249+255416 & $17.7 \pm 0.2$ & $0.8 \pm 0.3$ & GSHCTK J034249+253349 & $19.9 \pm 0.4$ & $-0.1 \pm 0.5$ \\
\hline GSHCTK J034249+254243 & $18.6 \pm 0.2$ & $1.4 \pm 0.4$ & GSHCTK J034250+252437 & $19.5 \pm 0.3$ & $0.7 \pm 0.4$ \\
\hline GSHCTK J034250+253334 & $18.7 \pm 0.2$ & $1.9 \pm 0.4$ & GSHCTK J034249+253801 & $19.0 \pm 0.2$ & $1.4 \pm 0.4$ \\
\hline GSHCTK J034249+254529 & $19.2 \pm 0.2$ & $0.5 \pm 0.4$ & GSHCTK J034250+253031 & $18.5 \pm 0.2$ & $1.0 \pm 0.3$ \\
\hline GSHCTK J034250+253810 & $18.7 \pm 0.2$ & $1.4 \pm 0.4$ & GSHCTK J034250+254456 & $18.6 \pm 0.2$ & $1.0 \pm 0.3$ \\
\hline GSHCTK J034250+252856 & $19.0 \pm 0.2$ & $1.3 \pm 0.4$ & GSHCTK J034250+254839 & $18.4 \pm 0.2$ & $1.1 \pm 0.3$ \\
\hline GSHCTK J034251+253759 & $18.6 \pm 0.2$ & $0.7 \pm 0.3$ & GSHCTK J034251+253048 & $18.4 \pm 0.2$ & $1.8 \pm 0.4$ \\
\hline GSHCTK J034251+253152 & $17.7 \pm 0.2$ & $1.6 \pm 0.3$ & GSHCTK J034251+254932 & $19.9 \pm 0.3$ & $0.9 \pm 0.6$ \\
\hline GSHCTK J034251+254352 & $18.0 \pm 0.2$ & $0.4 \pm 0.3$ & GSHCTK J034252+253427 & $18.3 \pm 0.2$ & $5.2 \pm 4.6$ \\
\hline GSHCTK J034251+252201 & $19.9 \pm 0.4$ & $-0.5 \pm 0.5$ & GSHCTK J034251+252240 & $19.2 \pm 0.3$ & $1.2 \pm 0.4$ \\
\hline GSHCTK J034252+252916 & $19.0 \pm 0.2$ & $0.4 \pm 0.3$ & GSHCTK J034252+254334 & $18.1 \pm 0.2$ & $0.3 \pm 0.3$ \\
\hline GSHCTK J034252+254445 & $19.5 \pm 0.3$ & $1.5 \pm 0.6$ & GSHCTK J034252+254633 & $18.8 \pm 0.2$ & $1.2 \pm 0.4$ \\
\hline GSHCTK J034252+252305 & $20.7 \pm 0.7$ & $-0.3 \pm 0.8$ & GSHCTK J034252+254029 & $18.6 \pm 0.2$ & $0.6 \pm 0.3$ \\
\hline GSHCTK J034252+252938 & $18.7 \pm 0.2$ & $0.6 \pm 0.3$ & GSHCTK J034252+254954 & $17.7 \pm 0.2$ & $0.7 \pm 0.3$ \\
\hline GSHCTK J034252+255352 & $21.1 \pm 0.9$ & $-1.6 \pm 0.9$ & GSHCTK J034252+254226 & $18.3 \pm 0.2$ & $1.4 \pm 0.4$ \\
\hline GSHCTK J034253+255332 & $19.7 \pm 0.3$ & $0.4 \pm 0.4$ & GSHCTK J034253+252026 & $22.1 \pm 3.0$ & $-2.3 \pm 3.0$ \\
\hline GSHCTK J034253+252953 & $21.1 \pm 0.8$ & $-0.8 \pm 0.9$ & GSHCTK J034253+253511 & $17.4 \pm 0.2$ & $0.8 \pm 0.3$ \\
\hline GSHCTK J034253+254947 & $18.5 \pm 0.2$ & $0.2 \pm 0.3$ & GSHCTK J034254+252424 & $18.0 \pm 0.2$ & $0.1 \pm 0.3$ \\
\hline GSHCTK J034254+254527 & $18.8 \pm 0.2$ & $1.0 \pm 0.4$ & GSHCTK J034254+255031 & $20.5 \pm 0.6$ & $-1.0 \pm 0.6$ \\
\hline GSHCTK J034254+255219 & $18.3 \pm 0.2$ & $1.4 \pm 0.4$ & GSHCTK J034254+252522 & $19.4 \pm 0.3$ & $0.5 \pm 0.4$ \\
\hline GSHCTK J034254+252513 & $19.1 \pm 0.2$ & $1.4 \pm 0.4$ & GSHCTK J034255+254215 & $19.5 \pm 0.3$ & $1.1 \pm 0.5$ \\
\hline GSHCTK J034255+252006 & $21.1 \pm 1.1$ & $-1.2 \pm 1.1$ & GSHCTK J034255+252535 & $20.1 \pm 0.4$ & $-0.1 \pm 0.5$ \\
\hline GSHCTK J034255+253515 & $19.5 \pm 0.3$ & $-0.4 \pm 0.4$ & GSHCTK J034255+255022 & $18.8 \pm 0.2$ & $0.4 \pm 0.3$ \\
\hline GSHCTK J034255+253646 & $18.8 \pm 0.2$ & $1.3 \pm 0.4$ & GSHCTK J034255+253825 & $20.4 \pm 0.5$ & $1.3 \pm 1.0$ \\
\hline GSHCTK J034255+254808 & $17.6 \pm 0.2$ & $-0.7 \pm 0.3$ & GSHCTK J034255+255252 & $20.1 \pm 0.4$ & $0.4 \pm 0.6$ \\
\hline GSHCTK J034256+252614 & $21.2 \pm 1.0$ & $-0.8 \pm 1.1$ & GSHCTK J034256+253019 & $18.6 \pm 0.2$ & $1.5 \pm 0.4$ \\
\hline GSHCTK J034256+252349 & $19.7 \pm 0.3$ & $0.2 \pm 0.4$ & GSHCTK J034256+255144 & $19.5 \pm 0.3$ & $0.4 \pm 0.4$ \\
\hline GSHCTK J034256+253249 & $19.2 \pm 0.2$ & $0.8 \pm 0.4$ & GSHCTK J034257+254259 & $19.8 \pm 0.3$ & $-0.7 \pm 0.4$ \\
\hline GSHCTK J034257+254146 & $18.8 \pm 0.2$ & $-0.0 \pm 0.3$ & GSHCTK J034257+252836 & $18.1 \pm 0.2$ & $0.8 \pm 0.3$ \\
\hline GSHCTK J034258+253628 & $21.4 \pm 1.1$ & $-1.6 \pm 1.2$ & GSHCTK J034258+254447 & $18.5 \pm 0.2$ & $0.5 \pm 0.3$ \\
\hline GSHCTK J034258+254918 & $18.1 \pm 0.2$ & $1.1 \pm 0.3$ & GSHCTK J034258+254427 & $17.7 \pm 0.2$ & $1.2 \pm 0.3$ \\
\hline GSHCTK J034258+252752 & $17.7 \pm 0.2$ & $0.8 \pm 0.3$ & GSHCTK J034259+252424 & $19.6 \pm 0.3$ & $-0.3 \pm 0.4$ \\
\hline GSHCTK J034259+255305 & $19.6 \pm 0.3$ & $1.1 \pm 0.5$ & GSHCTK J034259+252614 & $18.8 \pm 0.2$ & $0.8 \pm 0.4$ \\
\hline GSHCTK J034259+255042 & $19.7 \pm 0.3$ & $0.6 \pm 0.5$ & GSHCTK J034259+252758 & $19.1 \pm 0.2$ & $0.0 \pm 0.4$ \\
\hline GSHCTK J034259+253816 & $18.5 \pm 0.2$ & $1.0 \pm 0.4$ & GSHCTK J034259+252203 & $19.1 \pm 0.2$ & $0.9 \pm 0.4$ \\
\hline GSHCTK J034259+252630 & $18.9 \pm 0.2$ & $0.3 \pm 0.3$ & GSHCTK J034260+255416 & $17.9 \pm 0.2$ & $1.1 \pm 0.3$ \\
\hline GSHCTK J034260+253243 & $19.1 \pm 0.2$ & $0.8 \pm 0.4$ & GSHCTK J034260+255049 & $21.1 \pm 0.9$ & $-1.2 \pm 1.0$ \\
\hline GSHCTK J034260+255350 & $18.2 \pm 0.2$ & $0.7 \pm 0.3$ & GSHCTK J034260+252606 & $17.9 \pm 0.2$ & $1.1 \pm 0.3$ \\
\hline GSHCTK J034260+252136 & $18.9 \pm 0.2$ & $0.3 \pm 0.4$ & GSHCTK J034260+255201 & $18.8 \pm 0.2$ & $1.2 \pm 0.4$ \\
\hline GSHCTK J034300+254823 & $18.7 \pm 0.2$ & $0.9 \pm 0.4$ & GSHCTK J034300+255343 & $18.3 \pm 0.2$ & $0.7 \pm 0.3$ \\
\hline GSHCTK J034300+255148 & $18.0 \pm 0.2$ & $0.9 \pm 0.3$ & GSHCTK J034300+252636 & $21.0 \pm 0.9$ & $-1.2 \pm 0.9$ \\
\hline GSHCTK J034300+253123 & $19.5 \pm 0.3$ & $1.0 \pm 0.5$ & GSHCTK J034300+253424 & $18.2 \pm 0.2$ & $2.0 \pm 0.4$ \\
\hline GSHCTK J034300+255027 & $18.1 \pm 0.2$ & $1.1 \pm 0.3$ & GSHCTK J034301+254349 & $18.6 \pm 0.2$ & $0.7 \pm 0.3$ \\
\hline GSHCTK J034301+255055 & $18.1 \pm 0.2$ & $0.4 \pm 0.3$ & GSHCTK J034301+254956 & $19.1 \pm 0.2$ & $0.5 \pm 0.4$ \\
\hline GSHCTK J034301+252521 & $19.4 \pm 0.3$ & $0.5 \pm 0.4$ & GSHCTK J034301+254704 & $16.9 \pm 0.2$ & $0.7 \pm 0.3$ \\
\hline GSHCTK J034301+253002 & $20.1 \pm 0.4$ & $-0.4 \pm 0.5$ & GSHCTK J034301+253019 & $18.2 \pm 0.2$ & \\
\hline GSHCTK J034302+252101 & $18.9 \pm 0.2$ & $0.7 \pm 0.4$ & GSHCTK J034301+255016 & $18.4 \pm 0.2$ & $1.6 \pm 0.4$ \\
\hline GSHCTK J034302+252754 & $19.0 \pm 0.2$ & & GSHCTK J034302+255135 & & \\
\hline GSHCTK J034302+253654 & $18.1 \pm 0.2$ & $1.0 \pm 0.3$ & GSHCTK J034302+254513 & $19.7 \pm 0.3$ & $2.2 \pm 1.1$ \\
\hline GSHCTK J034302+253544 & $19.7 \pm 0.3$ & $-1.1 \pm 0.4$ & GSHCTK J034303+254947 & $17.4 \pm 0.2$ & \\
\hline GSHCTK J034303+253803 & $18.5 \pm 0.2$ & $1.2 \pm 0.4$ & GSHCTK J034303+252052 & $17.6 \pm 0.2$ & $0.8 \pm 0.3$ \\
\hline GSHCTK J034303+252829 & $17.8 \pm 0.2$ & $1.3 \pm 0.3$ & GSHCTK J034303+252145 & $18.4 \pm 0.2$ & \\
\hline GSHCTK J034303+253236 & $18.7 \pm 0.2$ & $0.4 \pm 0.3$ & GSHCTK J034303+253758 & $18.5 \pm 0.2$ & $1.1 \pm 0.3$ \\
\hline GSHCTK J034303+255046 & $17.6 \pm 0.2$ & $0.5 \pm 0.3$ & GSHCTK J034303+254440 & $19.0 \pm 0.2$ & $1.2 \pm 0.4$ \\
\hline GSHCTK J034303+254451 & $19.7 \pm 0.3$ & $0.7 \pm 0.5$ & GSHCTK J034304+253225 & $18.9 \pm 0.2$ & $0.6 \pm 0.4$ \\
\hline GSHCTK J034304+254409 & $18.6 \pm 0.2$ & $1.9 \pm 0.5$ & GSHCTK J034304+252933 & $18.0 \pm 0.2$ & $0.7 \pm 0.3$ \\
\hline GSHCTK J034304+253114 & $20.2 \pm 0.4$ & $-0.3 \pm 0.5$ & GSHCTK J034304+252143 & $18.0 \pm 0.2$ & $1.9=$ \\
\hline GSHCTK J034304+252205 & $18.6 \pm 0.2$ & $0.9 \pm 0.4$ & GSHCTK J034304+252541 & $18.4 \pm 0.2$ & $0.6 \pm 0.3$ \\
\hline
\end{tabular}




\begin{tabular}{|c|c|c|c|c|c|}
\hline Id & Imag & $R-I$ & Id & Imag & $R-I$ \\
\hline GSHCTK J034305+254737 & $23.3 \pm 7.3$ & $-3.7 \pm 7.3$ & GSHCTK J034305+254856 & $18.5 \pm 0.2$ & $1.1 \pm 0.4$ \\
\hline GSHCTK J034305+252116 & $17.1 \pm 0.2$ & $1.0 \pm 0.3$ & GSHCTK J034305+253143 & $18.3 \pm 0.2$ & $0.6 \pm 0.3$ \\
\hline GSHCTK J034305+252302 & $17.5 \pm 0.2$ & $1.8 \pm 0.3$ & GSHCTK J034306+253243 & $19.4 \pm 0.2$ & $1.5 \pm 0.5$ \\
\hline GSHCTK J034306+255312 & $21.6 \pm 2.3$ & $-1.5 \pm 2.4$ & GSHCTK J034308+253823 & $20.0 \pm 0.4$ & $0.3 \pm 0.6$ \\
\hline GSHCTK J034308+254354 & $19.0 \pm 0.2$ & $1.4 \pm 0.4$ & GSHCTK J034308+253309 & $19.3 \pm 0.2$ & $0.5 \pm 0.4$ \\
\hline GSHCTK J034308+254958 & $17.6 \pm 0.2$ & $0.9 \pm 0.3$ & GSHCTK J034308+253123 & $19.6 \pm 0.3$ & $-0.4 \pm 0.4$ \\
\hline GSHCTK J034309+253809 & $20.2 \pm 0.4$ & $-1.0 \pm 0.5$ & GSHCTK J034309+254336 & $18.2 \pm 0.2$ & $1.3 \pm 0.3$ \\
\hline GSHCTK J034309+253249 & $19.0 \pm 0.2$ & $0.8 \pm 0.4$ & GSHCTK J034309+253749 & $18.1 \pm 0.2$ & $1.0 \pm 0.3$ \\
\hline GSHCTK J034309+253229 & $19.3 \pm 0.3$ & $-0.0 \pm 0.4$ & GSHCTK J034309+254650 & $17.9 \pm 0.2$ & $1.2 \pm 0.3$ \\
\hline GSHCTK J034310+253442 & $18.8 \pm 0.2$ & $0.9 \pm 0.4$ & GSHCTK J034310+253749 & $18.4 \pm 0.2$ & $0.8 \pm 0.3$ \\
\hline GSHCTK J034310+252003 & $18.5 \pm 0.2$ & $1.0 \pm 0.4$ & GSHCTK J034310+252355 & $20.5 \pm 0.6$ & $1.1 \pm 1.0$ \\
\hline GSHCTK J034310+255330 & $18.1 \pm 0.2$ & $0.7 \pm 0.3$ & GSHCTK J034310+253614 & $18.3 \pm 0.2$ & $1.8 \pm 0.4$ \\
\hline GSHCTK J034310+254349 & $18.7 \pm 0.2$ & $0.6 \pm 0.3$ & GSHCTK J034310+253158 & $17.1 \pm 0.2$ & $1.2 \pm 0.3$ \\
\hline GSHCTK J034310+251948 & $17.7 \pm 0.2$ & $1.9 \pm 0.3$ & GSHCTK J034311+252225 & $18.7 \pm 0.2$ & $1.4 \pm 0.4$ \\
\hline GSHCTK J034311+253411 & $18.8 \pm 0.2$ & $2.1 \pm 0.5$ & GSHCTK J034311+254708 & $17.0 \pm 0.2$ & $0.9 \pm 0.3$ \\
\hline GSHCTK J034311+252105 & $18.4 \pm 0.2$ & $1.4 \pm 0.4$ & GSHCTK J034311+252203 & $17.5 \pm 0.2$ & $3.4 \pm 0.5$ \\
\hline GSHCTK J034311+254834 & $13.4 \pm 0.2$ & $0.6 \pm 0.3$ & GSHCTK J034312+252908 & $17.8 \pm 0.2$ & $1.0 \pm 0.3$ \\
\hline GSHCTK J034312+254947 & $17.9 \pm 0.2$ & $0.1 \pm 0.3$ & GSHCTK J034312+252229 & $17.9 \pm 0.2$ & $2.0 \pm 0.4$ \\
\hline GSHCTK J034313+253734 & $20.0 \pm 0.4$ & $0.0 \pm 0.5$ & GSHCTK J034313+253342 & $20.2 \pm 0.4$ & $-0.6 \pm 0.5$ \\
\hline GSHCTK J034313+252209 & $18.4 \pm 0.2$ & $1.6 \pm 0.4$ & $13+253026$ & $19.3 \pm 0.2$ & $0.5 \pm 0.4$ \\
\hline GSHCTK J034313+251924 & $17.2 \pm 0.2$ & $1.4 \pm 0.3$ & GSHCTK J034314+253311 & $16.4 \pm 0.2$ & $1.1 \pm 0.3$ \\
\hline GSHCTK J034314+254433 & $18.0 \pm 0.2$ & $0.8 \pm 0.3$ & GSHCTK J & $18.9 \pm 0.2$ & $1.1 \pm 0.4$ \\
\hline GSHCTK J0 & $18.6 \pm 0.2$ & $1.1 \pm 0.4$ & GSHCTK & $18.1 \pm 0.2$ & $1.3 \pm 0.3$ \\
\hline GSHCTK J034315+252908 & $17.1 \pm 0.2$ & $1.0 \pm 0.3$ & GSHCTK JC & $17.3 \pm 0.2$ & $0.8 \pm 0.3$ \\
\hline GSHCTK J034316+2: & $21.3 \pm 1.2$ & $-0.8 \pm 1.2$ & GSHC & $18.8 \pm 0.2$ & $1.6 \pm 0.4$ \\
\hline GSHCTK J0 & $17.9 \pm 0.2$ & $0.7 \pm 0.3$ & GSHC & $18.7 \pm 0.2$ & $1.3 \pm 0.4$ \\
\hline GSHCTK J0 & $19.3 \pm 0.2$ & $-0.1 \pm 0.4$ & $\mathrm{GSH}$ & $22.3 \pm 3.5$ & $-2.2 \pm 3.5$ \\
\hline GSHCTK J034 & $20.2 \pm 0.6$ & $-1.1 \pm 0.6$ & GSHC & $18.0 \pm 0.2$ & $0.8 \pm 0.3$ \\
\hline GSHCTK J034317+2 & $18.5 \pm 0.2$ & $-0.1 \pm 0.3$ & GSH & $19.2 \pm 0.2$ & $0.6 \pm 0.4$ \\
\hline GSHCTK J034317+252521 & $19.0 \pm$ & $0.8 \pm 0.4$ & GS & $18.0 \pm 0.2$ & $1.1 \pm 0.3$ \\
\hline GSHCTK J034317+252948 & $18.7 \pm 0.2$ & $1.4 \pm 0.4$ & 937 & $17.7 \pm 0.2$ & $1.0 \pm 0.3$ \\
\hline GSHCTK J034317+254927 & $17.4 \pm 0.2$ & $1.0 \pm 0.3$ & GSH & $19.5 \pm 0.4$ & $-0.1 \pm 0.5$ \\
\hline GSHCTK J034318+254935 & $17.8 \pm 0.2$ & $0.8 \pm 0.3$ & & $18.9 \pm$ & $0.5 \pm 0.4$ \\
\hline GSHCTK J03 & $20.1 \pm$ & $0.9 \pm 0.7$ & 27 & $18.5 \pm 0.2$ & $1.5 \pm 0.4$ \\
\hline GSHCTK J034318+253331 & $19.9 \pm 0.4$ & $0.1 \pm 0.5$ & GSH & $17.8 \pm 0.2$ & $1.3 \pm 0.3$ \\
\hline GSHCTK J034319+254537 & $19.9 \pm 0.4$ & $0.2 \pm 0.5$ & GSHCTK JC & $18.9 \pm 0.2$ & $0.1 \pm 0.4$ \\
\hline GSHCTK J034319+252503 & $17.9 \pm 0.2$ & $1.4 \pm 0.3$ & & $19.4 \pm 0.3$ & $-0.1 \pm 0.4$ \\
\hline GSHCTK J034319+252424 & $19.3 \pm 0.3$ & $1.0 \pm 0.4$ & 2933 & $19.5 \pm 0.3$ & $-0.4 \pm 0.4$ \\
\hline GSHCTK J034320+255141 & $17.6 \pm 0.2$ & $0.8 \pm 0.3$ & GSHCTK J0 & $17.9 \pm 0.2$ & $1.7 \pm 0.3$ \\
\hline GSHCTK J034320+252047 & $19.7 \pm 0.6$ & $0.5 \pm 0.7$ & 2207 & $17.8 \pm 0.2$ & $1.4 \pm 0.3$ \\
\hline GSHCTK J034321+252904 & $18.6 \pm 0.2$ & $0.5 \pm 0.3$ & GSHCTK & $18.5 \pm 0.2$ & $3.3 \pm 1.1$ \\
\hline GSHCTK J034321+254258 & $18.9 \pm 0.2$ & $0.8 \pm 0.4$ & $321+253911$ & $16.9 \pm 0.2$ & $0.8 \pm 0.3$ \\
\hline GSHCTK J034321+254322 & $18.4 \pm 0.2$ & $1.2 \pm 0.4$ & & $19.8 \pm 0.3$ & $-0.3 \pm 0.4$ \\
\hline GSHCTK J034321+252457 & $18.4 \pm 0.2$ & $0.7 \pm 0.3$ & GSHCTK J & $16.9 \pm 0.2$ & $0.5 \pm 0.3$ \\
\hline GSHCTK J034321+254645 & $18.5 \pm 0.2$ & $1.6 \pm 0.5$ & GSHCTK J0 & $19.6 \pm 0.3$ & $0.4 \pm 0.5$ \\
\hline GSHCTK J034321+252233 & $18.4 \pm 0.2$ & $1.0 \pm 0.4$ & 2740 & $18.7 \pm 0.2$ & $2.1 \pm 0.5$ \\
\hline GSHCTK J034322+253214 & $20.4 \pm 0.5$ & $-0.4 \pm 0.6$ & GSHCTK J0 & $17.5 \pm 0.2$ & $1.8 \pm 0.3$ \\
\hline GSHCTK J034322+255316 & $19.9 \pm 0.3$ & $1.3 \pm 0.6$ & GSHCTK J03 & $18.3 \pm 0.2$ & $1.2 \pm 0.4$ \\
\hline GSHCTK J034322+255019 & $18.3 \pm 0.2$ & $1.5 \pm 0.4$ & 5046 & $18.6 \pm 0.2$ & $1.4 \pm 0.4$ \\
\hline GSHCTK J034322+252835 & $17.9 \pm 0.2$ & $3.1 \pm 0.6$ & +253103 & $18.1 \pm 0.2$ & $0.9 \pm 0.3$ \\
\hline GSHCTK J034323+255017 & $18.5 \pm 0.2$ & $2.0 \pm 0.4$ & GSHCTK J03 & $17.7 \pm 0.2$ & $1.3 \pm 0.3$ \\
\hline GSHCTK J034323+253331 & $18.7 \pm 0.2$ & $1.6 \pm 0.4$ & 4948 & $17.9 \pm 0.2$ & $1.6 \pm 0.3$ \\
\hline GSHCTK J034324+254915 & $17.4 \pm 0.2$ & $0.7 \pm 0.3$ & $4+254942$ & $18.2 \pm 0.2$ & $1.4 \pm 0.4$ \\
\hline GSHCTK J034324+254322 & $18.1 \pm 0.2$ & $1.7 \pm 0.4$ & GSHCTK J034324+253129 & $20.0 \pm 0.4$ & $0.2 \pm 0.6$ \\
\hline GSHCTK J034324+255223 & $18.4 \pm 0.2$ & $1.5 \pm 0.4$ & +254415 & $17.4 \pm 0.2$ & $1.5 \pm 0.3$ \\
\hline GSHCTK J034324+255327 & $19.0 \pm 0.2$ & $0.6 \pm 0.3$ & $5+253010$ & $18.3 \pm 0.2$ & $1.9 \pm 0.4$ \\
\hline GSHCTK J034325+255404 & $18.9 \pm 0.2$ & $1.2 \pm 0.3$ & GSHCTK J034325+252505 & $19.1 \pm 0.3$ & $1.1 \pm 0.4$ \\
\hline GSHCTK J034325+253218 & $17.6 \pm 0.2$ & $0.5 \pm 0.3$ & GSHCTK J034326+253001 & $19.8 \pm 0.4$ & $0.1 \pm 0.5$ \\
\hline GSHCTK J034326+253032 & $18.3 \pm 0.2$ & $3.2 \pm 0.9$ & $34326+252158$ & $17.2 \pm 0.2$ & $1.5 \pm 0.3$ \\
\hline GSHCTK J034326+253426 & $19.9 \pm 0.4$ & $-1.1 \pm 0.5$ & GSHCTK J034326+253809 & $19.6 \pm 0.3$ & $0.7 \pm 0.5$ \\
\hline GSHCTK J034326+252919 & $23.5 \pm 9.3$ & $-3.7 \pm 9.3$ & GSHCTK J034326+254924 & $18.2 \pm 0.2$ & $0.6 \pm 0.3$ \\
\hline GSHCTK J034327+252147 & $18.6 \pm 0.4$ & $-0.2 \pm 0.5$ & GSHCTK J034327+254917 & $17.9 \pm 0.2$ & $1.9 \pm 0.4$ \\
\hline GSHCTK J034328+254437 & $18.5 \pm 0.2$ & $0.7 \pm 0.4$ & GSHCTK J034328+254506 & $19.0 \pm 0.2$ & $0.7 \pm 0.4$ \\
\hline GSHCTK J034328+254636 & $18.4 \pm 0.2$ & $0.9 \pm 0.4$ & GSHCTK J034328+253634 & $17.4 \pm 0.2$ & $0.8 \pm 0.3$ \\
\hline GSHCTK J034328+255353 & $16.7 \pm 0.2$ & $1.0 \pm 0.3$ & GSHCTK J034328+253714 & $19.0 \pm 0.2$ & $0.1 \pm 0.4$ \\
\hline GSHCTK J034328+253339 & $19.7 \pm 0.3$ & $-0.4 \pm 0.4$ & GSHCTK J034328+255101 & $19.1 \pm 0.2$ & $-0.0 \pm 0.4$ \\
\hline GSHCTK J034328+254056 & $18.4 \pm 0.2$ & $0.0 \pm 0.3$ & GSHCTK J034329+252713 & $18.3 \pm 0.2$ & $0.6 \pm 0.3$ \\
\hline GSHCTK J034329+253621 & $17.7 \pm 0.2$ & $0.7 \pm 0.3$ & GSHCTK J034329+254548 & $21.7 \pm 2.2$ & $-2.2 \pm 2.2$ \\
\hline GSHCTK J034329+251921 & $19.1 \pm 0.4$ & $-1.2 \pm 0.5$ & GSHCTK J034329+253541 & $19.9 \pm 0.3$ & $1.1 \pm 0.7$ \\
\hline GSHCTK J034330+253530 & $20.1 \pm 0.4$ & $0.6 \pm 0.6$ & GSHCTK J034330+253755 & $18.6 \pm 0.2$ & $0.6 \pm 0.3$ \\
\hline GSHCTK J034330+254906 & $18.5 \pm 0.2$ & $1.4 \pm 0.4$ & GSHCTK J034330+252521 & $17.6 \pm 0.2$ & $1.2 \pm 0.3$ \\
\hline GSHCTK J034330+252158 & $16.9 \pm 0.2$ & $1.2 \pm 0.3$ & GSHCTK J034331+253355 & $18.7 \pm 0.2$ & $3.4 \pm 1.6$ \\
\hline GSHCTK J034331+253755 & $18.7 \pm 0.2$ & $1.2 \pm 0.4$ & GSHCTK J034331+252908 & $18.9 \pm 0.2$ & $0.2 \pm 0.3$ \\
\hline GSHCTK J034331+254729 & $17.7 \pm 0.2$ & $1.3 \pm 0.3$ & GSHCTK J034332+255320 & $19.3 \pm 0.2$ & $0.6 \pm 0.4$ \\
\hline GSHCTK J034332+253749 & $18.4 \pm 0.2$ & $1.5 \pm 0.4$ & GSHCTK J034332+252020 & $18.8 \pm 0.3$ & $2.0 \pm 1.0$ \\
\hline GSHCTK J034332+254705 & $18.8 \pm 0.2$ & $1.5 \pm 0.6$ & GSHCTK J034332+253532 & $19.1 \pm 0.2$ & $1.7 \pm 0.5$ \\
\hline GSHCTK J034333+252932 & $19.0 \pm 0.2$ & $0.0 \pm 0.3$ & GSHCTK J034333+253724 & $19.6 \pm 0.3$ & $1.3 \pm 0.6$ \\
\hline GSHCTK J034334+254253 & $20.0 \pm 0.4$ & $0.1 \pm 0.6$ & GSHCTK J034334+252859 & $19.5 \pm 0.3$ & $1.6 \pm 0.6$ \\
\hline GSHCTK J034334+254001 & $19.1 \pm 0.3$ & $0.7 \pm 0.4$ & GSHCTK J034334+252923 & $18.7 \pm 0.2$ & $0.4 \pm 0.3$ \\
\hline GSHCTK J034334+253010 & $20.7 \pm 0.6$ & $-0.5 \pm 0.7$ & GSHCTK J034334+253740 & $17.8 \pm 0.2$ & $1.2 \pm 0.3$ \\
\hline GSHCTK J034334+253304 & $21.1 \pm 1.0$ & $-1.8 \pm 1.1$ & GSHCTK J034335+252757 & $19.8 \pm 0.3$ & $0.7 \pm 0.5$ \\
\hline GSHCTK J034335+254541 & $17.7 \pm 0.2$ & $0.6 \pm 0.3$ & GSHCTK J034335+254424 & $19.8 \pm 0.5$ & $-0.6 \pm 0.5$ \\
\hline GSHCTK J034336+253359 & $18.3 \pm 0.2$ & $0.3 \pm 0.3$ & GSHCTK J034336+253337 & $20.4 \pm 0.3$ & $-0.3 \pm 0.5$ \\
\hline
\end{tabular}




\begin{tabular}{lcc|lcc} 
Table A3 continued $\ldots$ \\
\hline Id & Imag & $R-I$ & Id & Imag & $R-I$ \\
\hline GSHCTK J034336+252343 & $21.0 \pm 0.4$ & $-1.4 \pm 0.5$ & GSHCTK J034336+254344 & $19.4 \pm 0.2$ & $-0.7 \pm 0.4$ \\
GSHCTK J034336+253941 & $18.9 \pm 0.2$ & $1.7 \pm 0.5$ & GSHCTK J034336+253603 & $18.5 \pm 0.2$ & $0.6 \pm 0.3$ \\
GSHCTK J034336+254220 & $18.3 \pm 0.2$ & $0.5 \pm 0.3$ & GSHCTK J034336+252605 & $19.8 \pm 0.2$ & $0.8 \pm 0.4$ \\
GSHCTK J034337+253638 & $20.5 \pm 0.3$ & $-1.0 \pm 0.4$ & GSHCTK J034337+254950 & $14.1 \pm 0.2$ & $2.4 \pm 0.3$
\end{tabular}

